\title{
Modeling, Analysis and Simulation of Nonperturbative Nonlinear Laser-Gas Interaction
}

by

\section{Marianna Lytova}

A thesis submitted to the Faculty of Graduate and Postdoctoral

Affairs in partial fulfillment of the requirements

for the degree of

Doctor of Philosophy

in

Mathematics $^{1}$

Carleton University

Ottawa, Ontario

(C) 2017

Marianna Lytova

\footnotetext{
${ }^{1}$ The PhD program is a joint program with University of Ottawa, administrated by the OttawaCarleton Institute for Mathematics and Statistics
} 


\section{Abstract}

In this thesis we study, analyze, extend and implement the nonperturbative nonlinear Maxwell-Schrödinger-Plasma (MASP) model, originally derived by Lorin et al. [78]. The model was developed to describe the high order optical nonlinearities and the low density free electron plasma generated due to laser field.

The MASP model has important advantages, it is based on the original, i.e. nonasymptotic, physical equations, and uses self-consistent description of the micro (quantum)- and macro (field)- variables. However, its major drawback is a high computational cost, which in practice means that only the shortest propagation lengths can be calculated. In order to reduce this cost, several extensions to this model were proposed and tested. One of these is discussed in the thesis: it is the MASP model enriched by a polarization evolution equation from its simplest version in a form of transport equation to more complex nonlinear variants. We show that homogeneous transport equation is a more universal tool to simulate the high harmonics spectra at shorter times and/or at a lower computational cost, while the nonlinear equation could be useful for modeling the pulse profiles when the ionization level is moderate. The gain associated with the considered modifications of the MASP model, being expressed in reduction of computational time and the number of processors involved, is 2-3 orders of magnitude. 


\section{Acknowledgements}

I am deeply grateful to my supervisor, Prof. Emmanuel Lorin for his guidance, support, criticism and patience, for his paramount professionalism both in research and teaching. Thank you for getting me involved in this interesting and vibrant area, for generous sharing of your ideas, experience and time.

This project was carried out in close collaboration with Prof. André D. Bandrauk from Université de Sherbrooke, to whom I extend my sincere gratitude.

Additionally, I thank Prof. David Amundsen and Prof. Yves Bourgault for their comments and positive criticism of my proposal and for their consent to read and review my thesis in spite of their busy schedule. I appreciate the time and efforts spent by the external examiner Prof. Thomas Brabec and other committee members, who agreed to contribute their diverse expertise and time for evaluating this research. With my pleasure I thank some incredible professors of the Ottawa-Carleton Institute for Mathematics and Statistics: Frithjof Lutscher, Natalia Stepanova, and again David Amundsen and Emmanuel Lorin, for their inspiring and very useful lectures. I thank Compute Canada for access to multiprocessor supercomputers for the most part of simulations reported here.

Last but not least, special thanks to my family, Dmitry and Misha, for their understanding, encouragement and help during all the long time of my graduate studies. I wish to express my gratitude to my parents and sister for their remote but very valuable support. 


\section{Contents}

$\begin{array}{ll}\text { Abstract } & \text { ii }\end{array}$

$\begin{array}{ll}\text { Acknowledgements } & \text { iii }\end{array}$

List of Tables $\quad$ vii

List of Figures $\quad x$

Appendices $\quad x i$

List of Acronyms xiii

List of Common Symbols $\quad$ Xv

1 Introduction 1

1.1 Motivation and Organization of the Thesis . . . . . . . . 1

1.2 Introduction to Nonlinear Optics . . . . . . . . . . . . . 4

1.3 Perturbative Processes . . . . . . . . . . . . . . . . . . 11

1.3.1 Third-Harmonic Generation and Kerr Effect . . . . . . . . . 11

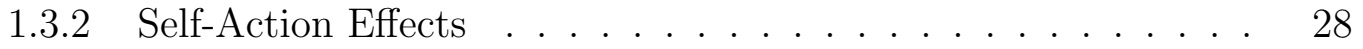

1.4 Nonperturbative Processes . . . . . . . . . . . . . . . . . 34

1.4.1 High Harmonic Generation . . . . . . . . . . . . . . . . 34

1.4.2 Laser Filamentation: Standard Models. . . . . . . . . . . . . . 44 
2 Maxwell-Schrödinger-Plasma Models $\quad 55$

2.1 Introductory Remarks . . . . . . . . . . . . . . . . 55

2.2 Maxwell-Schrödinger-Plasma Model . . . . . . . . . . . . . . 56

$2.2 .1 \quad$ Initial Laser Pulse . . . . . . . . . . . . . . . . . . . . 62

2.3 Polarization Evolution Equation for Ultrashort Pulses . . . . . . . . 68

2.3.1 Simple Evolution Equation for the Dipole Moment _ . . . . 69

2.3.2 General Evolution Equation for the Dipole Moment . . . . . . 72

2.3.3 Global Model Under the Paraxial Approximation . . . . . . 79

2.4 Existence and Uniqueness of Weak Solutions for the MASP Models . 84

2.4 .1 "Pure" MASP Model . . . . . . . . . . . . . . . . . . . . 85

2.4.2 MASP Model Supplemented by Evolution Equations for Polarization and Free Electron Density . . . . . . . . . . . . 98

3 Numerical Methods for Solving the MASP Model 104

3.1 Discretization of the 1d Maxwell Equations . . . . . . . . . . . . 104

3.1 .1 Numerical Scheme I . . . . . . . . . . . . . . . . . . . 106

3.1 .2 Numerical Scheme II . . . . . . . . . . . . . . . . . . . 112

3.1.3 Propagating of the LP-pulse: Perturbative Approach _ . . . . 115

3.2 Discretization of 3d Maxwell's Equations . . . . . . . . . . . . 117

3.2 .1 Yee's Numerical Scheme . . . . . . . . . . . . . . . . . . 117

3.2.2 Mur's Absorbing Boundary Conditions . . . . . . . . . . 120

3.3 Discretization of the $2 \mathrm{~d}$ Schrödinger Equation $\ldots \ldots \ldots \ldots$

3.3.1 Symmetric Strang Splitting _. . . . . . . . . . . . 126

3.3.2 Numerical Scheme for the Schrödinger Equation with Timeindependent Potential _ . . . . . . . . . . . . . . . . 129

3.3.3 Boundary Condition Problem . . . . . . . . . . . . . . 134

3.3.4 Solving of the Matrix Equation _. . . . . . . . . . . 142

3.4 Discretization of the Polarization Evolution Equation . . . . . . 143 
3.5 Integrating of Drude's Equation . . . . . . . . . . . . . 147

3.6 Parallel Computing Aspects . . . . . . . . . . . . . . . . . . . . 149

3.6.1 Parallelism in the Case of 1d-2d MS Model . . . . . . . . . 149

3.6.2 Parallelism in the Case of 1d-2d Model Enriched by the Polarization Evolution Equation . . . . . . . . . . . . . 150

3.6.3 Parallelism for 3d-1d/2d Model . . . . . . . . . . . . . . 152

4 Applications of the MS and MASP Models 154

4.1 1d-2d MS Model . . . . . . . . . . . . . . . . . . 154

4.1.1 Introductory Remarks and Motivation . . . . . . . . . 154

4.1.2 Geometry of the Model and Calculation Parameters. . . . . . 157

4.1 .3 Physical Results . . . . . . . . . . . . . . . . . . . 160

4.2 1d-2d MASP Model with the Polarization Evolution Equation . . . 169

4.2.1 Testing the Models in Perturbative Regime . . . . . . . . . . 169

4.2.2 MS and MASP with 1d Polarization Equation . . . . . . . . 177

4.3 3d-1d Enriched MASP Model . . . . . . . . . . . . . . . . 195

4.3.1 Including the Polarization Equation . . . . . . . . . . . 196

5 Summary of Findings and Further Directions 200

$\begin{array}{ll}\text { Appendices } & 203\end{array}$

$\begin{array}{ll}\text { A Atomic units } & 204\end{array}$

B Basic Facts About the Nonlinear Schrödinger Equation 206

$\begin{array}{ll}\text { C Proof of Lemma 2.1.1 } & 209\end{array}$

$\begin{array}{ll}\text { Bibliography } & 214\end{array}$ 


\section{List of Tables}

3.1 Compressed Row Storage format for sparse matrixes . . . . . . . . . . 142

4.1 Propagation time and $L^{2}$-norm of the wavefunction. . . . . . . . 163

4.2 Order of convergence. . . . . . . . . . . . . . . . . 174

4.3 Model and computational parameters. . . . . . . . . . . . . . 180 


\section{List of Figures}

1.1 Energy level diagram of an atom. . . . . . . . . . . . . . . 9

1.2 Third-Harmonic Generation. . . . . . . . . . . . . . . . . 11

1.3 One- and two-photon resonant contributions. . . . . . . . . . . . 15

1.4 The phase mismatch factor. . . . . . . . . . . . . . . 23

1.5 Intensity of the third harmonic. . . . . . . . . . . . . . . . 24

1.6 Parameters of a Gaussian beam. . . . . . . . . . . . . . . . 26

1.7 Self-focusing it the Kerr medium. . . . . . . . . . . . . . . . . 29

1.8 Shape of the typical HHG spectra. . . . . . . . . . . . . . . . 35

1.9 Semi-classical model for HHG for hydrogen atom. . . . . . . . . . . . 36

1.10 Factor $\Upsilon$ in the expression for the kinetic energy... . . . . . . . . . . 38

1.11 Solutions of the semi-classical theory. . . . . . . . . . . . . . . . 38

1.12 HHG: atom response in time and spectrum. . . . . . . . . . . . . 40

1.13 Laser filamentation: qualitative model. . . . . . . . . . . . . 45

2.1 Spatial domains for the MASP model. . . . . . . . . . . . . . . 59

2.2 Propagation of ultrashort laser pulse. . . . . . . . . . . . 62

$2.3 \mathrm{CP}$ initial laser pulse. . . . . . . . . . . . . . . 64

2.4 Initial LP-laser pulses . . . . . . . . . . . . . . . . . 65

$2.51 \mathrm{~d}-2 \mathrm{~d}$ geometry of the computational model . . . . . . . . . . 67

2.6 Macroscopic model with initial data from TDSE. . . . . . . . . . . . 70 
2.7 Spatial evolution of polarization. . . . . . . . . . . . . . . . 71

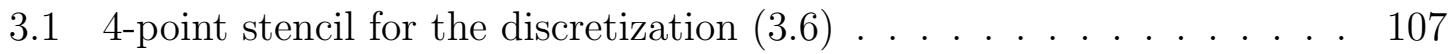

3.2 Staggered grid for Yee's scheme . . . . . . . . . . . . . . . . 118

3.3 Symmetry Strang splitting scheme. . . . . . . . . . . . . . 127

3.4 Recast space grid. . . . . . . . . . . . . . . . . . . . . . 130

3.5 Structure of the matrix $A_{R} \ldots \ldots \ldots \ldots$. . . . . . . . 133

3.6 Structure of the matrixes $a_{R} \ldots \ldots \ldots \ldots$. . . . . . . . 133

3.7 Transmission, Reflection and Absorption coefficients. . . . . . . . . 136

3.8 Boundary with CAP. . . . . . . . . . . . . . . . . . 137

3.9 Example of parallelization: 1d-2d MS model. . . . . . . . . . . . . . . 149

3.10 Parallelization: 1d-2d MASP model with the polarization equation. . 151

3.11 Computational geometry of 3d-(1d/2d/3d) MASP model. . . . . . . . 152

3.12 Example of parallelization: 3d-(1d/2d/3d) enriched MASP model. . . 153

4.1 Homogeneous and non-homogeneous density profiles. . . . . . . . . . 158

4.2 Reflection of $E_{y}$-component at the entering border. . . . . . . . 158

4.3 Intensity of the low order electric field harmonics, LP pulse. . . . . . 160

4.4 Intensity of harmonics of the dipole moment, LP-pulse . . . . . . . . 161

4.5 Intensity of harmonics of the transmitted electric field. . . . . . . . . 162

4.6 Intensity of the low order harmonics vs. propagation length, LP-pulse. 162

4.7 Low order harmonic spectrum, CP-pulse. . . . . . . . . . . . . . . . 164

4.8 Low order harmonic of the dipole moment, CP-pulse. . . . . . . . . . 164

4.9 Total harmonic spectrum, CP-pulse. . . . . . . . . . . . 165

4.10 The level sets of electron density. . . . . . . . . . . . . . . 166

4.11 Intensity of the low order harmonics vs. propagation length, CP-pulse. 167

4.12 The measure the circularity of the polarization vector. . . . . . . . 168

4.13 Transmitted electric field as a function of space, Models 1 and 2. . . . 171 


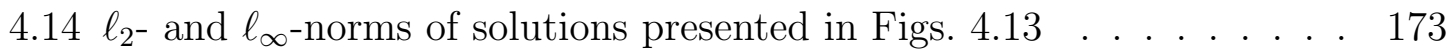

$4.15 \ell_{2}$-norms for solutions presented in Figs. $4.13 \ldots \ldots$. . . . . . . . 173

4.16 Electric field and spectra for Model 2. . . . . . . . . . . . . . 175

4.17 Intensity of the third harmonic: Models 1 and 2. . . . . . . . . . 176

4.18 Spectral intensities of the electric field harmonics. . . . . . . . . . . . 178

4.19 Results of the MASP model enriched by the transport equation . . . 179

4.20 Reference results of the MASP model. . . . . . . . . . . . . . . . . . 181

4.21 MASP vs. enriched MASP models: electric field; . . . . . . . . . . . . 183

$4.22 \ell_{2^{-}}$and $\ell_{\infty}$-norms for solutions presented in Fig. $\left.4.21 \mathrm{a}\right) \quad \ldots . . . . \quad 184$

$4.23 \ell_{2^{-}}$and $\ell_{\infty^{-n o r m s}}$ of solutions presented in Fig. 4.21 b) . . . . . . 185

4.24 MASP vs. enriched MASP models: electric field; dense gas. . . . . . . 186

$4.25 \ell_{2^{-}}$and $\ell_{\infty}$-norms for solutions presented in Fig. $4.24 \ldots \ldots$

4.26 MASP vs. enriched MASP models: spectra. . . . . . . . . . . . . . . 189

4.27 MASP vs. enriched MASP models: difference in spectra. . . . . . . . 190

4.28 MASP vs. enriched MASP models: spectra; high initial intensity. . . 191

4.29 MASP vs. enriched MASP models: differences in spectra; high intensity.191

4.30 Spectra of high harmonics: different domain decompositions. . . . . . 192

4.31 Difference in spectra presented in Figs. 4.30 . . . . . . . . . . 193

4.32 Calculated intensity of the Gaussian beam in plane $(x, z) \ldots$. . . . 196

4.33 Gaussian pulse width in transversal section . . . . . . . . . . . . . 196

4.34 3D "pure" MASP vs. enriched MASP models . . . . . . . . . . . . 197

4.35 Intensity profiles of the beam after propagation . . . . . . . . . 198

B.1 Self-steepening and optical shock formation. . . . . . . . . . . 208 


\section{Appendices}

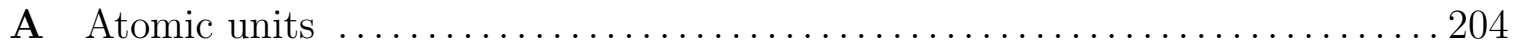

B Basic Facts About the Nonlinear Schrödinger Equation ............... 206

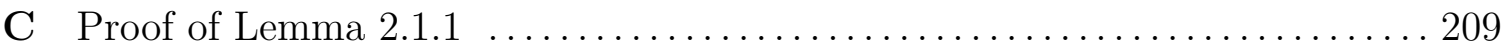




\title{
List of Acronyms
}

\author{
ABCs - Absorbing Boundary Conditions \\ ADI - Alternative Direction Implicit (method) \\ a.u. - atomic units \\ BCs - Boundary Conditions \\ BOA - Born-Oppenheimer Approximation \\ CAP - Complex Absorbing Potential \\ c.c. - complex conjugate \\ CP - Circularly Polarized \\ EM - Electromagnetic \\ FDS - Finite Difference Scheme \\ FDM - Finite Difference Method \\ FDTD - Finite-Difference Time Domain (method) \\ FT - Fourier Transform \\ GMKP - Generalized Modified Kadomtsev-Petviashvili (equation) \\ HHG - High Harmonic Generation \\ HOKE - Higher Order Kerr Effect \\ HOMO - Highest Occupied Molecular Orbital \\ ICs - Initial conditions \\ KdV - Korteveg-de Vries (equation) \\ LHS - Left-Hand Side
}


LP - Linearly Polarized

MASP - Maxwell-Schrödinger-Plasma (model)

MBEs - Maxwell-Bloch equations

MEs - Maxwell Equations

MHOHG - Molecular High Order Harmonic Generation

MKP - Modified Kadomtsev-Petviashvili (equation)

MPP - Multi-Photon Process

MS - Maxwell-Schrödinger (model)

NLSE - Nonlinear Schrödinger Equation

ODE - Ordinary Differential Equation

PDE - Partial Differential Equation

RHS - Right-Hand Side

SAE - Single Active Electron (approximation)

SFA - Strong Field Approximation

SI - International System of Units

SVEA - Slowly Varying Envelope Approximation

TDSE - Time-Dependent Schrödinger Equation

THG - Third Harmonic Generation

UPPE - Unidirectional Pulse Propagation Equation

w.r.t. - with respect to 


\title{
List of Common Symbols
}

\author{
Part I: Mathematical symbols \\ $\Omega \quad$ Spatial domain for Maxwell's equations \\ $\omega_{i} \quad i^{\text {th }}$ spatial domain for Schrödinger equation \\ $\left.\begin{array}{l}\mathcal{D} \\ \mathcal{C}_{0}^{\infty}\end{array}\right\}$ Set of test functions \\ $\mathcal{D}^{\prime} \quad$ Set of distributions \\ $\mathcal{C}^{n} \quad$ Set of functions differentiable up to the order $n$ \\ $\mathcal{C}_{b}^{n} \quad$ Set of bounded $\mathcal{C}^{n}$ functions \\ $L^{p} \quad$ Lebesgue space of order $p$ \\ $H^{k} \quad$ Hilbert space of order $k$ \\ $W^{k, p} \quad$ Sobolev space of order $\{k, p\}$ \\ $\nabla \quad$ Nabla operator \\ $\nabla_{h} \quad$ Discrete nabla operator \\ $\triangle \quad$ Laplace operator \\ $u \quad$ Heaviside step function \\ $\delta \quad$ Dirac delta function
}




\section{Part II: Physical symbols}

$\begin{array}{ll}c & \text { speed of light } \\ \hbar & \text { reduced Planck constant } \\ e & \text { elementary charge } \\ \varepsilon_{0} & \text { vacuum permittivity } \\ \alpha & \text { fine-structure constant } \\ m_{e} & \text { electron mass } \\ m_{p} & \text { proton mass } \\ \mathbf{E} & \text { electric field } \\ \mathbf{D} & \text { electric displacement } \\ \mathbf{H} & \text { magnetic field } \\ \mathbf{B} & \text { magnetic induction } \\ \mathbf{J} & \text { current density } \\ \mathbf{P} & \text { polarization } \\ \mathbf{d} & \text { dipole moment } \\ A & \text { Complex amplitude of electric field } \\ \chi_{i j k l \ldots \ldots}^{(m)} & \text { susceptibility }(m+1) \text {-rank tensor } \\ \varepsilon_{i j k l \ldots}^{(m)} & \text { dielectric permittivity }(m+1) \text {-rank tensor } \\ \psi, \Psi & \text { wavefunctions } \\ V_{C} & \text { Coulombian potential } \\ R_{0} & \text { internuclear distance in molecular ion } \mathrm{H}_{2}^{+}\end{array}$




\section{Chapter 1}

\section{Introduction}

\subsection{Motivation and Organization of the Thesis}

Modern laser technology allows for the generation of ultrafast laser pulses with intensities exceeding the internal electric field in atoms and molecules [12,24]. The interaction of such pulses with atomic or molecular gas activates some nonperturbative processes, such as, the high harmonic generation [35] (see the Section 1.4.1) or above threshold ionization [105]. Thus obtained short high-frequency pulses are used in attophysics as a tool to study the dynamics and control of interatomic electrons, whose scale of motion is of the same order as the pulse duration, i.e. attoseconds ( 1 asec $\left.=10^{-18} \mathrm{~s}\right)$. Another highly nonlinear phenomenon appearing in nonperturbative regime is laser filamentation [12] (see the Section 1.4.2), whose potential fascinating applications require rigorously derived mathematical equations to model the parameters involved in this complex process.

The principal subject of the thesis is the analysis, the study and the implementation of improved non-standard nonperturbative nonlinear Maxwell-SchrödingerPlasma (MASP) models, originally developed by E. Lorin et al. in 2007 [79], and developed in $[75,80-82]$ with the aim of simulating high order nonlinearities in a gas. 
In contrast to standard models (e.g., NEE, UPPE, see further Section 1.4.2), this one is partially $a b$ initio in the sense of the quantum description of the molecular gas, which computes in a self-consistent way the atoms/molecules/ions response to the incident laser pulse, and the evolution of this pulse as a result of interaction with thus polarized medium. However, from a practical point of view, the MASP model confronts a major issue, which is a huge computational cost for the evaluation of the nonlinear polarization using time-dependent Schrödinger equations (TDSEs), see the Section 2.2. Realistic simulations are then only possible on very short propagation distances (not exceeding $1 \mathrm{~mm}[80]$ ), which at first sight makes the model impractical for studying filamentation. In this thesis we improve the MASP model by incorporating into the model an additional equation describing the polarization vector evolution. This supplementary equation allows to reduce the number of TDSEs to be solved (at defined length of the pulse propagation), and then reduces the overall computational complexity of the numerical model.

The thesis is organized as follows. Chapter 1 presents the introductory material and provides an overview of some nonlinear optics phenomena (e.g. third harmonic generation, self-focusing, high harmonics generation, laser filamentation, etc.), as well as examples of their mathematical descriptions, which are useful in the light of our investigations. This chapter is mainly based on the canonical textbooks, monographs as well as the contemporary reviews. The derivation of the basic equations and formulae follows Boyd [24], Newell and Moloney [94], Landau and Lifshitz [68-70], and Shen [106]. We also used the articles and reviews, most often with reference to Bergé et al. [21], Kolesik and Moloney [62], Panagiotopoulos et al. [98], Corkum [35], Lewenstein et al. [73], Newell [93].

In Chapter 2 we present the Maxwell-Schrödinger-Plasma model and propose its improvement in the form of an evolution equation for the polarization vector. We describe geometry of the numerical models and discuss the question about existence 
and uniqueness of the solutions. Chapter 2 is partially based on the original articles by Lorin et al. $[77,79,81,83,84]$. The author of this thesis co-authored the two last papers.

Chapter 3 is devoted to the numerical implementation of the MASP model. We present respective finite difference schemes and provide their analysis of convergence for those cases where it is possible to do analytically. The chapter also covers the computational and parallelization aspects, which are fundamental in the MASP modeling.

Chapter 4 provides examples of the models applications to some physical phenomena. Particular attention is paid to the simulation of short circularly polarized laser pulse, which is currently a hot topic in the nonlinear optics community. Section 4.1 mainly follows the article by Lytova, Lorin and Bandrauk [85].

In Sections 4.2 and 4.3 we provides examples of the $1 \mathrm{~d}-2 \mathrm{~d}$ and $3 \mathrm{~d}-1 \mathrm{~d}$ enriched MASP models application. We compare the results of the improved and the pure MASP models computing $\ell_{2^{-}}$and $\ell_{\infty^{-}}$discrete norms of the solutions.

Chapter 5 contains a conclusion and outlooks.

Throughout this thesis, equations and formulae are written in atomic units, see Appendix A, unless otherwise specially stated. 


\subsection{Introduction to Nonlinear Optics}

We start with an overview of basic important facts in nonlinear optics and some mathematical methods used in this area, following the classic books $[24,70,94,106]$. The interaction between intense electromagnetic (EM) field and medium can be characterized by two main feature of nonlinearity. First, intense light pulse can induce multi-photon processes, e.g., generation of high order harmonics (with reference to frequency of the falling pulse), while linear optics studies only single-photon processes. Second, in response to the propagation of intense pulse in a medium, the properties of latter, in particular, refractive index, change, while in linear optics the refractive index is considered as independent of the intensity.

In an electrically neutral dielectric medium, the spatial separation of charges takes place under the influence of the electric field. At the microscopic level, a measure that characterizes this separation is the dipole moment of the system (e.g. atom or molecule), which is defined in classical (in the sense "not quantum") theory as [68]

$$
\mathbf{d}=\sum_{i=1}^{N} e_{i} \mathbf{r}_{i}
$$

where $e_{i}$ and $\mathbf{r}_{i}$ are the charge and the radius-vector of the $i^{\text {th }}$ particle, and the summation is over all $N$ particles of the system, i.e. nuclei and electrons.

At the macroscopic level, considering the small enough unit of volume $\Delta V$, which still contains a number $\Delta N$ atoms or molecules with the number density $\mathcal{N}=$ $\lim _{\Delta V \rightarrow 0} \frac{\Delta N}{\Delta V}$, the polarization vector is defined as the sum over all of $\Delta N$ the microscopic dipole moments divided by unit of this volume [70]

$$
\mathbf{P}=\lim _{\Delta V \rightarrow 0} \frac{1}{\Delta V} \sum_{l} \mathbf{d}_{l}=\lim _{\Delta V \rightarrow 0} \frac{\Delta N}{\Delta V} \frac{1}{\Delta N} \sum_{l} \mathbf{d}_{l} \equiv \mathcal{N} \mathbf{d}_{\mathrm{av}}
$$


where $\mathbf{d}_{\mathrm{av}}$ denotes the dipole moment averaged over $\Delta N$ molecular systems. Thus, the formulae (1.1) (or more precisely, its quantization) and (1.2) define the procedure of calculation of the polarization vector through the microscopic parameters of the system. For example, such knowledge can be obtained from the numerical solution to the Heisenberg or Schrödinger equations for an atomic or molecular system placed in an external electric field (for more details, see the Section 2.2). However, the above procedure is not in general feasible in practice, usually due to its high computational cost. Computationally cheaper approximate methods that also allow to calculate the polarization have been developed for practical purposes. Let us consider the ideas underlying these methods.

The induced time-dependent polarization vector $\mathbf{P}(\mathbf{x}, t)$ at point $\mathbf{x}=(x, y, z)$ can be expressed as a function of the external electric field $\mathbf{E}(\mathbf{x}, t)$. When the external field is weak enough, the relation can be expressed in the linear form:

$$
\mathbf{P}(\mathbf{x}, t)=\chi^{(1)} \mathbf{E}(\mathbf{x}, t),
$$

where the proportionality factor $\chi^{(1)}$ is referred to as the instantaneous linear susceptibility. Let us remark that in general case, the linear susceptibility is a second-rank tensor, but as we are studying here the processes in a gas, i.e. medium invariant with respect to (w.r.t.) spatial inversion, the coefficient $\chi^{(1)}$ in the expression of the linear polarization can be treated as a scalar (see further (1.21) and (1.22)). Also, certainly the assumption of the immediate medium response is not realistic. Later, in Section 1.3.1, we will see how to bring into consideration the effect of the retarded response, but first we are interested in very general formulation of the problem.

If the field is strong enough so that the mentioned above nonlinear effects occur, the expression for the polarization vector must be corrected. It is reasonable to 
generalize (1.3) as a power expansion:

$$
\mathbf{P}(\mathbf{x}, t)=\mathbf{P}^{(1)}(\mathbf{x}, t)+\mathbf{P}^{(2)}(\mathbf{x}, t)+\mathbf{P}^{(3)}(\mathbf{x}, t)+\ldots
$$

so that the $i^{\text {th }}$ component $(i=1,2,3)$ of order $m$ reads:

$$
P_{i}^{(m)}(\mathbf{x}, t)=\chi_{m+1}^{(m)} \underbrace{E_{j k}(\mathbf{x}, t) E_{k}(\mathbf{x}, t) \ldots}_{m},
$$

implying summation over repeated indices $j, k, \ldots$, which independently take the values from $\{1,2,3\}$. In other words, $\chi^{(m)}$ are tensors of rank $(m+1)$. The expression becomes much simpler in the case of centrosymmetric media, when all the evennumbered coefficients $\chi^{(2 m)}$ vanish identically, while the odd susceptibility tensors show high degree of symmetry and contain a certain number of zero-elements $[24,70$, 94]. Indeed, the second order nonlinearity reads as

$$
P_{i}^{(2)}(\mathbf{x}, t)=\chi_{i j k}^{(2)} E_{j}(\mathbf{x}, t) E_{k}(\mathbf{x}, t)
$$

while in case of central inversion (also known as point reflection), if any component of applied electric field changes its sign, then the sign of the corresponding polarization also must change to the opposite:

$$
-P_{i}^{(2)}(\mathbf{x}, t)=\chi_{i j k}^{(2)}\left[-E_{j}(\mathbf{x}, t)\right]\left[-E_{k}(\mathbf{x}, t)\right]=\chi_{i j k}^{(2)} E_{j}(\mathbf{x}, t) E_{k}(\mathbf{x}, t) .
$$

From (1.6) and (1.7) we can conclude that $P_{i}^{(2)}(\mathbf{x}, t) \equiv 0$. Obviously, the same reasoning can be applied to any even correction $P_{i}^{(2 m)}(m=2,3, \ldots)$. Then we get for the polarization vector instead of (1.4) the expansion for the odd terms only:

$$
\mathbf{P}(\mathbf{x}, t)=\mathbf{P}^{(1)}(\mathbf{x}, t)+\mathbf{P}^{(3)}(\mathbf{x}, t)+\ldots
$$


and for consistency the required tensor coefficients $\chi^{(2 m+1)}$, must be calculated within of quantum theory. Further, we briefly describe the technique by the example of Schrödinger equation, which is applicable in case of nonresonant response of the relevant micro-system [24].

The evolution of time-dependent wavefunction of atomic or molecular system with one outer electron $\psi(\mathbf{x}, t)$, which is subject to an external time-dependent electric field $\mathbf{E}(\mathbf{x}, t)$, can be described through the TDSE

$$
\mathrm{i} \partial_{t} \psi(\mathbf{x}, t)=\left[H_{0}(\mathbf{x})+V(\mathbf{x}, t)\right] \psi(\mathbf{x}, t),
$$

with the functional operator Hamiltonian $H(\mathbf{x})=H_{0}(\mathbf{x})+V(\mathbf{x}, t)$ written as the sum of (i) the time-independent Hamiltonian of an isolated atom: $H_{0}(\mathbf{x})=-\frac{1}{2} \triangle_{\mathbf{x}}+V_{C}(\mathbf{x})$, where $\triangle_{\mathbf{x}}$ is the Laplace operator, $V_{C}(\mathbf{x})$ is an electron-atomic core potential, and (ii) the time-dependent interaction operator $[24,69]$ :

$$
V(\mathbf{x}, t)=-\mathbf{d} \cdot \mathbf{E}(\mathbf{x}, t)
$$

where the electric dipole moment operator $\mathbf{d}=-\mathbf{x}$, since for the electron $e=-1$ a.u. In such a situation, there is no stationary state for (1.9), and the problem is to calculate in some way the time-dependent wavefunction $\psi(\mathbf{x}, t)$ in order to compute the microscopic dipole moment as the quantum-mechanical expectation value, i.e. the quantum average ( $d \mathbf{x}$ stands for the volume integration and the symbol "asterisk" denotes complex conjugation):

$$
\mathbf{d}(t)=-\int_{\mathbb{R}^{3}} \psi^{*}(\mathbf{x}, t) \mathbf{x} \psi(\mathbf{x}, t) d \mathbf{x}
$$

and finally, for the given density $\mathcal{N}(\mathbf{x})$, obtain the polarization $\mathbf{P}(\mathbf{x}, t)=\mathcal{N}(\mathbf{x}) \mathbf{d}(t)$, see (1.2). As we mentioned above, in general case the problem is computationally 
expensive, but under certain conditions it could be solved even using analytical approaches.

Namely, we can employ the perturbation theory $[19,24,69]$ by presenting the Hamiltonian as $H(\mathbf{x})=H_{0}(\mathbf{x})+\lambda V(\mathbf{x}, t)$, hence, we will search the wavefunction in the form of a power series in some small positive parameter $\lambda(1 \geq \lambda>0)$ : $\psi(\mathbf{x}, t)=\sum_{n=0}^{\infty} \lambda^{n} \psi^{(n)}(\mathbf{x}, t)$, and we can truncate the theoretically "infinite" series at the needed order. Then, assuming that the atom is initially in the ground state denoted as $u_{g}$, and applying the method of variable coefficients, the solution is obtained as [24]:

$$
\begin{aligned}
& \psi^{(0)}(\mathbf{x}, t)=u_{g}(\mathbf{x}) e^{-\mathbf{i} \mathcal{E}_{g} t}, \\
& \psi^{(n)}(\mathbf{x}, t)=\sum_{l} a_{l}^{(n)}(t) u_{l}(\mathbf{x}) e^{-\mathbf{i} \mathcal{E}_{l} t},
\end{aligned}
$$

where $\mathcal{E}_{g}$ and $u_{g}(\mathbf{x})$ are the ground state eigenvalue and eigenfunction of the operator $H_{0}(\mathbf{x}): H_{0}(\mathbf{x}) u_{g}(\mathbf{x})=\mathcal{E}_{g} u_{g}(\mathbf{x})$, while $u_{l}(\mathbf{x})$ and $\mathcal{E}_{l}$ refer to every other $l^{\text {th }}$ eigenstate of $H_{0}(\mathbf{x}): H_{0}(\mathbf{x}) u_{l}(\mathbf{x})=\mathcal{E}_{l} u_{l}(\mathbf{x})$; thus, $l=0$ corresponds to the ground state, $u_{g}(\mathbf{x}):=$ $u_{0}(\mathbf{x})$. The $n^{\text {th }}$-order contribution to the wavefunction denotes as $\psi^{(n)}(\mathbf{x}, t)$, so that, each of the time-dependent coefficients $a_{l}^{(n)}(t)$ could be computed from the equations

$$
\mathrm{i} \partial_{t} \psi^{(n)}(\mathbf{x}, t)=H_{0}(\mathbf{x}) \psi^{(n)}(\mathbf{x}, t)+V(\mathbf{x}, t) \psi^{(n-1)}(\mathbf{x}, t), \quad n=1,2,3 \ldots
$$

Now using the wavefunction (1.12), and setting accordingly (1.9) $\lambda=1$, we come to

$$
\begin{aligned}
\mathbf{d}^{(1)}(t)= & -\left[\int_{\mathbb{R}^{3}} \psi^{(0) *}(\mathbf{x}, t) \mathbf{x} \psi^{(1)}(\mathbf{x}, t) d \mathbf{x}+\int_{\mathbb{R}^{3}} \psi^{(1) *}(\mathbf{x}, t) \mathbf{x} \psi^{(0)}(\mathbf{x}, t) d \mathbf{x}\right], \\
\mathbf{d}^{(3)}(t)= & -\left[\int_{\mathbb{R}^{3}} \psi^{(0) *}(\mathbf{x}, t) \mathbf{x} \psi^{(3)}(\mathbf{x}, t) d \mathbf{x}+\int_{\mathbb{R}^{3}} \psi^{(1) *}(\mathbf{x}, t) \mathbf{x} \psi^{(2)}(\mathbf{x}, t) d \mathbf{x}+\right. \\
& \left.+\int_{\mathbb{R}^{3}} \psi^{(2) *}(\mathbf{x}, t) \mathbf{x} \psi^{(1)}(\mathbf{x}, t) d \mathbf{x}+\int_{\mathbb{R}^{3}} \psi^{(3) *}(\mathbf{x}, t) \mathbf{x} \psi^{(0)}(\mathbf{x}, t) d \mathbf{x}\right],
\end{aligned}
$$

and so on, for all required $\mathbf{d}^{(2 m+1)}(t)$ and then $\mathbf{P}^{(2 m+1)}(\mathbf{x}, t)$. Hence by applying (1.5), the corresponding tensors $\chi^{(2 m+1)}$ could also be obtained [24]. 


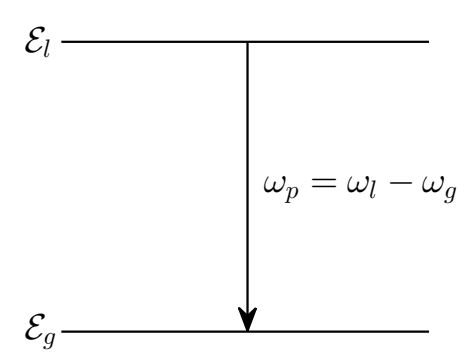

FiguRE 1.1: Energy level diagram of an atom. The resonance conditions: frequency of the nonforbidden transition between two levels $\mathcal{E}_{g}$ and $\mathcal{E}_{l}$ coincides with the frequency of the external field $\omega_{p}$ (in atomic units $\mathcal{E}_{g, l}=\omega_{g, l}$ ).

It is worth mentioning that the time-dependent coefficients $a_{l}^{(n)}(t)$ have a resonance form, that is they blow-up if at least one of the harmonics frequencies, $\omega_{p}$, of the external field, represented as a Fourier series: $\mathbf{E}(\mathbf{x}, t)=\sum_{p} \widehat{\mathbf{E}}\left(\mathbf{x}, \omega_{p}\right) e^{-\mathrm{i} \omega_{p} t}$ with amplitudes $\widehat{\mathbf{E}}\left(\mathbf{x}, \omega_{p}\right)$ (see, (1.19)), is equal to the frequency of any allowed transition in the atom or molecule, see Fig. 1.1. For example, at a first order one obtains [24]

$$
a_{l}^{(n)}(t)=-\sum_{p} \frac{\mathbf{x}_{l g} \cdot \widehat{\mathbf{E}}\left(\mathbf{x}, \omega_{p}\right)}{\omega_{l}-\omega_{g}-\omega_{p}} e^{i\left(\omega_{l}-\omega_{g}-\omega_{p}\right) t}
$$

where the matrix element of the vector $\mathbf{x}_{k l}=\int_{\mathbb{R}^{3}} u_{k}^{*}(\mathbf{x}) \mathbf{x} u_{l}(\mathbf{x}) d \mathbf{x}$ is called the electric dipole transition moment of the corresponding matrix. Thus, the condition of applicability of the perturbation theory in the first order writes as

$$
\left|\mathbf{x}_{l g} \cdot \widehat{\mathbf{E}}\left(\mathbf{x}, \omega_{p}\right)\right| \ll\left|\omega_{l}-\omega_{g}-\omega_{p}\right|, \quad \forall l, p \in \mathbb{N},
$$

for all eigenstates of an atom and harmonics frequencies of the external field.

Obtained according to (1.13) the first order contribution to the dipole moment is

$$
\mathbf{d}^{(1)}=\sum_{p} \sum_{l}\left(\frac{\mathbf{x}_{g l}\left(\mathbf{x}_{l g} \cdot \widehat{\mathbf{E}}\left(\mathbf{x}, \omega_{p}\right)\right)}{\omega_{l}-\omega_{g}-\omega_{p}}+\frac{\left(\mathbf{x}_{g l} \cdot \widehat{\mathbf{E}}\left(\mathbf{x}, \omega_{p}\right)\right) \mathbf{x}_{l g}}{\omega_{l}-\omega_{g}+\omega_{p}}\right) e^{-i \omega_{p} t}
$$


from which applying (1.5) the corresponding susceptibility tensor (see (1.18)), $\widehat{P}_{i}^{(1)}\left(\omega_{p}\right)=$ $\chi_{i j}^{(1)}\left(\omega_{p}\right) \widehat{E}_{j}\left(\mathbf{x}, \omega_{p}\right)$, is given by

$$
\chi_{i j}^{(1)}\left(\omega_{p}\right)=\mathcal{N} \sum_{l}\left(\frac{x_{g l}^{i} x_{l g}^{j}}{\omega_{l}-\omega_{g}-\omega_{p}}+\frac{x_{g l}^{j} x_{l g}^{i}}{\omega_{l}-\omega_{g}+\omega_{p}}\right)
$$

where the superscript of $x$ defines vector components $(i, j) \in\{1,2,3\}^{2}$. Similarly, the following order terms of the polarization vector can be calculated, hence the corresponding susceptibility tensors could be found [24]. Let us remark that the obtained $m^{\text {th }}$-order correction to the dipole moment $\mathbf{d}^{(m)}$ contains the multiplications $\underbrace{E_{j} E_{k} \ldots}_{m}$ under the summation sign, which is consistent with expansion (1.4), and expressions for its components (1.5).

To sum up, if the perturbation theory is valid for solving the TDSE (1.9) or other relevant microscopic equation, we can compute the convergent expansion (1.8) up to the desired term. The scope of the optical effects, which can be described in that regime is called perturbative nonlinear optics. 


\subsection{Perturbative Processes}

Since it is essential for further consideration, in this section we represent how the perturbative techniques treat nonlinear phenomena such as the third-harmonic generation (THG), the optical Kerr effect and the so-called self-action effects $[24,70,94,106]$.

\subsubsection{Third-Harmonic Generation and Kerr Effect}

THG is a process in which three photons of frequency $\omega$ are combined to generate one photon of frequency $3 \omega$. It is an example of so called multiphoton process (MPP). An important difference between MPP and single-photon processes is that the transition of an electron in an atom or molecule cannot be explained in terms of transition between the initial and final real physical states. Thus, about THG process we can say that an electron passes through three virtual levels, which may, but usually does not necessarily coincide with the real quantum levels, see Fig. 1.2, [24].

There is no contradiction with the fundamental principles of quantum mechanics, as according to Heisenberg's uncertainty principle, the lifetime in each virtual quantum state is $\delta t \approx \hbar / \delta \mathcal{E}$ (here we use SI units), where $\delta \mathcal{E}$ is the "distance" to the nearest real energy level [24]. If during this time the system absorbs a second photon, it goes to the next (maybe also virtual) level. In other words, MPPs have nonthreshold er probability

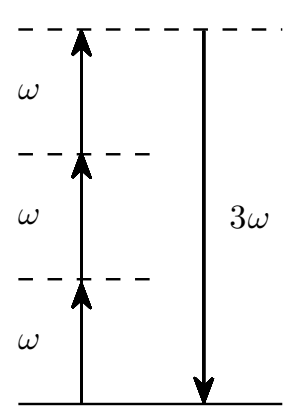

Figure 1.2: Third-Harmonic Generation: $\omega+\omega+\omega=3 \omega$. The dashed lines represent the virtual levels, see [24]. 
of the interaction between a quantum system and a photon. For crude estimation of $\delta t$, let us take $\delta \mathcal{E}=\hbar \omega$ with a frequency $\omega$ corresponding to infrared region of wavelengths, e.g. $\lambda=800 \mathrm{~nm}$, then $\delta t=\lambda / 2 \pi c \approx 4 \cdot 10^{-16}$ s. Since $\delta t$ is so extremely small, extremely high intensities are needed to run the detectable MPPs, which can be achieved with lasers only. For example, a ultrashort laser pulse with intensity $I=10^{14} \mathrm{~W} / \mathrm{cm}^{2}$ generates the photon flux $I / \hbar \omega \approx 4 \cdot 10^{32}$ photons $/\left(\mathrm{cm}^{2} \cdot \mathrm{s}\right)$, which allows to observe MPPs having used the contemporary methods of detection.

Before presenting the results about THG, let us recall the generalization of decomposition (1.8) in the case of a medium with dispersion and loss. To take into account the time delays, $\tau$ and $\tau_{i}(i=1,2,3 \ldots)$, between the external field action and the response of the medium, we rewrite (1.8) complemented by (1.5) as (see e.g. [24], [94]):

$$
\begin{aligned}
& P_{i}(\mathbf{x}, t)=\int_{-\infty}^{\infty} \chi_{i j}^{(1)}(\mathbf{x}, t-\tau) E_{j}(\mathbf{x}, \tau) d \tau+ \\
& +\int_{-\infty}^{\infty} \chi_{i j k l}^{(3)}\left(\mathbf{x}, t-\tau_{1}, t-\tau_{2}, t-\tau_{3}\right) E_{j}\left(\mathbf{x}, \tau_{1}\right) E_{k}\left(\mathbf{x}, \tau_{2}\right) E_{l}\left(\mathbf{x}, \tau_{3}\right) d \tau_{1} d \tau_{2} d \tau_{3}+\ldots
\end{aligned}
$$

where:

$$
\begin{aligned}
& \chi_{i j}^{(1)}(\mathbf{x}, t-\tau)=0, \quad t<\tau, \\
& \chi_{i j k l}^{(3)}\left(\mathbf{x}, t-\tau_{1}, t-\tau_{2}, t-\tau_{3}\right)=0, \quad t<\max _{i=1,2,3}\left\{\tau_{i}\right\},
\end{aligned}
$$

since the response takes some time (causality). In (1.16) $j, k, l$ and so on independently taking the values 1, 2 or 3 . Then the Fourier transform (FT) of the linear polarization reads

$$
\widehat{P}_{i}^{(1)}(\mathbf{x}, \omega)=\hat{\chi}_{i j}^{(1)}(\mathbf{x}, \omega) \widehat{E}_{j}(\mathbf{x}, \omega)
$$

where

$$
\widehat{\mathbf{P}}^{(1)}(\mathbf{x}, \omega)=\int_{-\infty}^{\infty} \mathbf{P}^{(1)}(\mathbf{x}, t) e^{i \omega t} d t
$$




$$
\begin{aligned}
\widehat{\mathbf{E}}(\mathbf{x}, \omega) & =\int_{-\infty}^{\infty} \mathbf{E}(\mathbf{x}, t) e^{\mathrm{i} \omega t} d t, \\
\hat{\chi}_{i j}^{(1)}(\mathbf{x}, \omega) & =\int_{-\infty}^{\infty} \chi_{i j}^{(1)}(\mathbf{x}, t) e^{\mathrm{i} \omega t} d t=\int_{0}^{\infty} \chi_{i j}^{(1)}(\mathbf{x}, t) e^{\mathrm{i} \omega t} d t,
\end{aligned}
$$

and the last equality holds because of causality, see (1.16). Now in case of centrosymmetric medium, omitting for notation simplicity the arguments $(\mathbf{x}, \omega)$, we get $[24,94,106]$ :

$$
\hat{\chi}_{i j}^{(1)}=\hat{\chi}_{11}^{(1)} \delta_{i j} \equiv \hat{\chi}^{(1)} \delta_{i j}
$$

where $\delta_{i j}$ is the Kronecker function. Hence we can rewrite (1.17) as

$$
\widehat{\mathbf{P}}^{(1)}(\mathbf{x}, \omega)=\hat{\chi}^{(1)}(\mathbf{x}, \omega) \widehat{\mathbf{E}}(\mathbf{x}, \omega) .
$$

Similarly from (1.15) and (1.16) we obtain

$$
\widehat{P}_{i}^{(3)}(\mathbf{x}, \omega)=\hat{\chi}_{i j k l}^{(3)}\left(\mathbf{x}, \omega_{1}, \omega_{2}, \omega_{3}\right) \widehat{E}_{j}\left(\mathbf{x}, \omega_{1}\right) \widehat{E}_{k}\left(\mathbf{x}, \omega_{2}\right) \widehat{E}_{l}\left(\mathbf{x}, \omega_{3}\right),
$$

where

$$
\hat{\chi}_{i j k l}^{(3)}\left(\mathbf{x}, \omega_{1}, \omega_{2}, \omega_{3}\right)=\iiint_{0}^{\infty} \chi_{i j k l}^{(3)}\left(\mathbf{x}, t_{1}, t_{2}, t_{3}\right) e^{\mathrm{i}\left(\omega_{1} t_{1}+\omega_{2} t_{2}+\omega_{3} t_{3}\right)} d t_{1} d t_{2} d t_{3} .
$$

Again, for centrosymmetric medium, omitting the common arguments $\left(\mathbf{x}, \omega_{1}, \omega_{2}, \omega_{3}\right)$ one can express the four-rank susceptibility tensor as:

$$
\hat{\chi}_{i j k l}^{(3)}=\hat{\chi}_{1122}^{(3)} \delta_{i j} \delta_{k l}+\hat{\chi}_{1212}^{(3)} \delta_{i k} \delta_{j l}+\hat{\chi}_{1221}^{(3)} \delta_{i l} \delta_{j k}
$$

i.e. out of the all $3^{4}=81$ tensor elements only $C_{3}^{1}+3 A_{3}^{2}=21$ elements are nonzero (here symbols $C_{3}^{1}$ used for combinations, and $A_{3}^{2}$ for partial permutations). Moreover, in general only 3 of these tensor elements are independent: $\hat{\chi}_{1122}^{(3)}, \hat{\chi}_{1212}^{(3)}$ and $\hat{\chi}_{1221}^{(3)}$. 
First, consider the term related to THG, when $\omega_{i}=\omega$ for all $i \in\{1,2,3\}$, so that $\sum_{i=1}^{3} \omega_{i}=3 \omega$. Then the FT of the corresponding susceptibility tensor is (because of the integrand symmetry (1.24) w.r.t. $t_{1}, t_{2}$ and $t_{3}$ here $\left.\hat{\chi}_{1122}^{(3)}=\hat{\chi}_{1212}^{(3)}=\hat{\chi}_{1221}^{(3)}\right)$

$$
\begin{aligned}
\hat{\chi}_{i j k l}^{(3)}(\mathbf{x}, 3 \omega) & =\iiint_{0}^{\infty} \chi_{i j k l}^{(3)}\left(\mathbf{x}, t_{1}, t_{2}, t_{3}\right) e^{\mathrm{i} \omega\left(t_{1}+t_{2}+t_{3}\right)} d t_{1} d t_{2} d t_{3}= \\
& =\hat{\chi}_{1122}^{(3)}(\mathbf{x}, 3 \omega)\left(\delta_{i j} \delta_{k l}+\delta_{i k} \delta_{j l}+\delta_{i l} \delta_{j k}\right), \quad 1 \leqslant i, j, k, l \leqslant 3,
\end{aligned}
$$

and after substitution into (1.23) it results in

$$
\widehat{P}_{i}(\mathbf{x}, 3 \omega)=\hat{\chi}_{1111}^{(3)}(\mathbf{x}, 3 \omega)(\widehat{\mathbf{E}}(\mathbf{x}, \omega) \cdot \widehat{\mathbf{E}}(\mathbf{x}, \omega)) \widehat{E}_{i}(\mathbf{x}, \omega),
$$

where $(\widehat{\mathbf{E}} \cdot \widehat{\mathbf{E}})=\widehat{E}_{j} \widehat{E}_{k} \delta_{j k}$ is the inner product, and the tensor element $\hat{\chi}_{1111}^{(3)}=\hat{\chi}_{1122}^{(3)}+$ $\hat{\chi}_{1212}^{(3)}+\hat{\chi}_{1221}^{(3)}=3 \hat{\chi}_{1122}^{(3)}$. Denoting thereafter in the text the only independent element of the tensor as $\hat{\chi}^{(3)}(\mathbf{x}, 3 \omega) \equiv \hat{\chi}_{111}^{(3)}(\mathbf{x}, 3 \omega)$, we finally obtain

$$
\widehat{\mathbf{P}}^{(3)}(\mathbf{x}, 3 \omega)=\hat{\chi}^{(3)}(\mathbf{x}, 3 \omega)(\widehat{\mathbf{E}}(\mathbf{x}, \omega) \cdot \widehat{\mathbf{E}}(\mathbf{x}, \omega)) \widehat{\mathbf{E}}(\mathbf{x}, \omega)
$$

However, THG is not the only possible outcome of the three-photon interaction, other possibilities result in the final harmonic frequency $\sum_{i=1}^{3} \omega_{i}=\omega$. It can be represented by three distinct permutations of the frequencies $(\omega, \omega,-\omega)$ and corresponds to susceptibility tensor

$$
\begin{aligned}
& \hat{\chi}_{i j k l}^{(3)}(\mathbf{x}, \omega)=\iiint_{0}^{\infty} \chi_{i j k l}^{(3)}\left(\mathbf{x}, t_{1}, t_{2}, t_{3}\right) e^{\mathrm{i} \omega\left(t_{1}+t_{2}-t_{3}\right)} d t_{1} d t_{2} d t_{3}= \\
& =\hat{\chi}_{1122}^{(3)}(\mathbf{x}, \omega)\left(\delta_{i j} \delta_{k l}+\delta_{i k} \delta_{j l}\right)+\hat{\chi}_{1221}^{(3)} \delta_{i l} \delta_{j k},
\end{aligned}
$$

expressed now through two independent elements $\hat{\chi}_{1122}=\hat{\chi}_{1212}$ and $\hat{\chi}_{1221}$, since here symmetry holds w.r.t. $t_{1}$ and $t_{2}$ only. Taking into account 3 possible permutations leading to the final frequency $\omega$, we obtain for the corresponding polarization: 


$$
\begin{aligned}
\widehat{\mathbf{P}}^{(3)}(\mathbf{x}, \omega)=\hat{\chi}_{1}^{(3)}(\mathbf{x}, \omega) & (\widehat{\mathbf{E}}(\mathbf{x}, \omega) \cdot \widehat{\mathbf{E}}(\mathbf{x},-\omega)) \widehat{\mathbf{E}}(\mathbf{x}, \omega)+ \\
& +\hat{\chi}_{2}^{(3)}(\mathbf{x}, \omega)(\widehat{\mathbf{E}}(\mathbf{x}, \omega) \cdot \widehat{\mathbf{E}}(\mathbf{x}, \omega)) \widehat{\mathbf{E}}(\mathbf{x},-\omega),
\end{aligned}
$$

where the scalar coefficients $\hat{\chi}_{1}^{(3)}(\mathbf{x}, \omega) \equiv 6 \hat{\chi}_{1122}^{(3)}(\mathbf{x}, \omega)$ and $\hat{\chi}_{2}^{(3)}(\mathbf{x}, \omega) \equiv 3 \hat{\chi}_{1221}^{(3)}(\mathbf{x}, \omega)$ are expressed through two non-zero independent elements of the tensor.

The quantum-mechanical calculations for the susceptibilities in (1.28) demon-

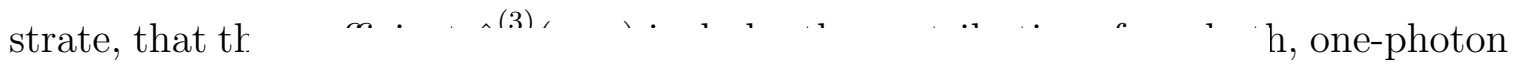
and two-phot ribution from two-photon $\mathrm{re}$

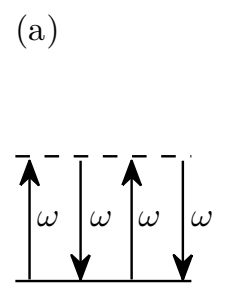

(b)

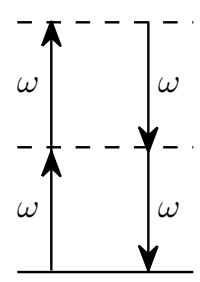

Figure 1.3: Energy level diagrams for (a) one-photon resonant contribution and (b) twophoton resonant contribution into the tensor of nonlinear susceptibility (1.27), see [24].

Note that the electric field $\mathbf{E}(\mathbf{x}, t)$ and the polarization $\mathbf{P}(\mathbf{x}, t)$ are physically measurable quantities, i.e. they both must be real values, and their FTs offer the following properties: $\widehat{\mathbf{E}}(\mathbf{x}, \omega)=\widehat{\mathbf{E}}^{*}(\mathbf{x},-\omega), \widehat{\mathbf{P}}^{(1)}(\mathbf{x}, \omega)=\widehat{\mathbf{P}}^{(1) *}(\mathbf{x},-\omega), \widehat{\mathbf{P}}^{(3)}(\mathbf{x}, 3 \omega)=$ $\widehat{\mathbf{P}}^{(3) *}(\mathbf{x},-3 \omega)$ and so on. From the real-valued field and polarization follow the realvalued coefficients $\chi^{(m)}(\mathbf{x}, t)(m \in \mathbb{N})$ and hence $\hat{\chi}^{(1)}(\mathbf{x}, \omega)=\hat{\chi}^{(1) *}(\mathbf{x},-\omega), \hat{\chi}_{1}^{(3)}(\mathbf{x}, \omega)=$ $\hat{\chi}_{1}^{(3) *}(\mathbf{x},-\omega), \hat{\chi}_{2}^{(3)}(\mathbf{x}, \omega)=\hat{\chi}_{2}^{(3) *}(\mathbf{x},-\omega), \hat{\chi}^{(3)}(\mathbf{x}, 3 \omega)=\hat{\chi}^{(3) *}(\mathbf{x},-3 \omega)$ or in general:

$$
\hat{\chi}^{(m)}\left(\mathbf{x}, \sum_{i=1}^{m} \omega_{i}\right)=\hat{\chi}^{(m) *}\left(\mathbf{x},-\sum_{i=1}^{m} \omega_{i}\right) .
$$

Now from the definitions (1.20) and (1.25), we can conclude that not only the linear susceptibility $\hat{\chi}^{(1)}(\mathbf{x}, \omega)$, but also the cubic one $\hat{\chi}^{(3)}(\mathbf{x}, 3 \omega)$ are complex analytic functions in the upper half of the complex plane $\omega=\operatorname{Re} \omega+i \operatorname{Im} \omega$, i.e., infinitely 
differentiable and single-valued if $\operatorname{Im} \omega>0$. For example, in case of $\hat{\chi}^{(1)}(\mathbf{x}, \omega)$, the integrand in $(1.20)$ is $\chi^{(1)}(\mathbf{x}, t) \exp [\mathrm{i}(\operatorname{Re} \omega) t-(\operatorname{Im} \omega) t]$, where the real factor $\exp [-$ $(\operatorname{Im} \omega) t]$ provides the integrability for $\operatorname{Im} \omega>0, t>0$. The same argument is valid for $\hat{\chi}^{(3)}(\mathbf{x}, 3 \omega)$, see $(1.25)$. Thus the Kramers-Kronig relations, which are valid in linear optics, can be also valid for some nonlinear processes, in particular, for the THG process [24]:

$$
\begin{aligned}
& \hat{\chi}^{(1)}(\omega)=\frac{1}{i \pi} \int_{-\infty}^{\infty} \frac{\hat{\chi}^{(1)}\left(\omega^{\prime}\right)}{\omega^{\prime}-\omega} d \omega^{\prime}, \\
& \hat{\chi}^{(3)}(3 \omega)=\frac{1}{i \pi} \int_{-\infty}^{\infty} \frac{\hat{\chi}^{(3)}\left(3 \omega^{\prime}\right)}{\omega^{\prime}-\omega} d \omega^{\prime} .
\end{aligned}
$$

On the other hand from $(1.27)$, we can see that $\hat{\chi}_{1,2}^{(3)}(\mathbf{x}, \omega)$ are not analytic in the upper half (also, obviously in the lower half) of the $\omega$-complex plane, as the integral diverges because of the presence of the factor $\exp \left(-\operatorname{Im} \omega\left(t_{1} \mp t_{2} \pm t_{3}\right)\right)$, and hence for $\hat{\chi}_{1,2}^{(3)}(\mathbf{x}, \omega)$ Kramers-Kronig relations are not valid [24].

As an intermediate conclusion notice, that the approach presented (1.15) is more general (in comparison to (1.8)) and results in the frequency-dependent nonlinear susceptibilities, which in general are complex quantities. The coefficient $\hat{\chi}^{(1)}(\omega)$ in (1.22) ties the complex amplitudes of the electric field and the polarization, and its imaginary part defines the absorbtion coefficient of the medium (see later (1.44)). Recall also, that when the Kramers-Kronig relations hold, one can determine the frequency dependence of the refractive index using the known absorption spectrum, which usually is easier to measure [24].

We start studying the nonlinear effects stimulated by propagation of an intense EM pulse in the electro-optic medium from the wave equation for electric field, which can be derived from Maxwell's equations (MEs). Namely, with the standard designation for the electric field $\mathbf{E}=\mathbf{E}(\mathbf{x}, t)$, the electric displacement $\mathbf{D}=\mathbf{D}(\mathbf{x}, t)$, the magnetic field $\mathbf{H}=\mathbf{H}(\mathbf{x}, t)$, the magnetic induction $\mathbf{B}=\mathbf{B}(\mathbf{x}, t)$, the density of free charges $\rho=\rho(\mathbf{x}, t)$, the current density of free charges $\mathbf{J}=\mathbf{J}(\mathbf{x}, t)$, the system reads 


$$
\left\{\begin{array}{l}
\nabla \cdot \mathbf{D}=4 \pi \rho \\
\nabla \cdot \mathbf{B}=0 \\
\nabla \times \mathbf{E}=-c^{-1} \partial_{t} \mathbf{B} \\
\nabla \times \mathbf{B}=c^{-1} \partial_{t} \mathbf{D}+4 \pi c^{-1} \mathbf{J}
\end{array}\right.
$$

In this Section, we consider the medium without free charges and currents: $\rho=0, \mathbf{J}=$ 0 . Also we assume that magnetic permeability $\mu=1$, so the medium is nonmagnetic corresponding to $\mathbf{B}=\mathbf{H}$. Then it follows from the two last equations of the system

$$
\nabla \times \nabla \times \mathbf{E}+c^{-2} \partial_{t}^{2} \mathbf{D}=0
$$

Recall that according to the constitutive relation, the displacement field can be expressed as $\mathbf{D}=\mathbf{E}+4 \pi \mathbf{P}$ and, as is the convention [24], we split the polarization vector $\mathbf{P}$, into its linear and nonlinear parts:

$$
\mathbf{P}=\mathbf{P}^{(1)}+\mathbf{P}^{N L}
$$

so that the linear part of the displacement field is

$$
\mathbf{D}^{(1)}=\mathbf{E}+4 \pi \mathbf{P}^{(1)}
$$

Note that $\nabla \times \nabla \times \mathbf{E}=\nabla(\nabla \cdot \mathbf{E})-\triangle \mathbf{E}$, and the equation $\nabla \cdot \mathbf{D}=0$ does not guarantee $\nabla \cdot \mathbf{E}=0$, in general case. For isotropic materials, applying (1.22), we can write

$$
\nabla \cdot \mathbf{E}=-\left(1+4 \pi \chi^{(1)}\right)^{-1} \nabla \cdot \mathbf{P}^{N L}
$$

which is not 0 in general. However, in the case of specific geometry, such as infinite plane wave $\nabla \cdot \mathbf{E}=\mathbf{i} \mathbf{k} \cdot \mathbf{E}=0$, when the nonlinearity is not extremely strong, this term can be considered as negligible, and degenerates into the following wave equation [24] 


$$
\partial_{t}^{2} \mathbf{E}-c^{2} \triangle \mathbf{E}=-4 \pi \partial_{t}^{2} \mathbf{P}
$$

For the general case of a dispersive and dissipative medium, we must consider each frequency component of the field separately. Following [24], we represent $\mathbf{E}, \mathbf{D}^{(1)}$ and $\mathbf{P}^{N L}$ as the sum of their various frequency components:

$$
\begin{aligned}
\mathbf{E}(\mathbf{x}, t) & =\sum_{n \geq 1} \mathbf{E}_{n}(\mathbf{x}, t), \\
\mathbf{D}^{(1)}(\mathbf{x}, t) & =\sum_{n \geq 1} \mathbf{D}_{n}^{(1)}(\mathbf{x}, t), \\
\mathbf{P}^{N L}(\mathbf{x}, t) & =\sum_{n \geq 1} \mathbf{P}_{n}^{N L}(\mathbf{x}, t),
\end{aligned}
$$

where each component could be represented in terms of the complex amplitudes:

$$
\begin{aligned}
\mathbf{E}_{n}(\mathbf{x}, t) & =\mathbf{E}_{n}(\mathbf{x}) e^{-\mathrm{i} \omega_{n} t}+\mathbf{E}_{n}^{*}(\mathbf{x}) e^{\mathrm{i} \omega_{n} t} \\
\mathbf{D}_{n}^{(1)}(\mathbf{x}, t) & =\mathbf{D}_{n}^{(1)}(\mathbf{x}) e^{-\mathrm{i} \omega_{n} t}+\mathbf{D}_{n}^{(1) *}(\mathbf{x}) e^{\mathrm{i} \omega_{n} t} \\
\mathbf{P}_{n}^{N L}(\mathbf{x}, t) & =\mathbf{P}_{n}^{N L}(\mathbf{x}) e^{-\mathrm{i} \omega_{n} t}+\mathbf{P}_{n}^{N L *}(\mathbf{x}) e^{\mathrm{i} \omega_{n} t}
\end{aligned}
$$

Note that here and further the complex amplitudes $\mathbf{E}_{n}(\mathbf{x}) \equiv \widehat{\mathbf{E}}\left(\mathbf{x}, \omega_{n}\right)$ as well as $\mathbf{D}_{n}^{(1)}(\mathbf{x}) \equiv \widehat{\mathbf{D}}^{(1)}\left(\mathbf{x}, \omega_{n}\right)$ and $\mathbf{P}_{n}^{N L}(\mathbf{x}) \equiv \widehat{\mathbf{P}}^{N L}\left(\mathbf{x}, \omega_{n}\right)$. Now from (1.34), we can write

$$
\mathbf{D}_{n}^{(1)}(\mathbf{x})=\left(1+4 \pi \hat{\chi}^{(1)}\left(\omega_{n}\right)\right) \mathbf{E}_{n}(\mathbf{x}) \equiv \hat{\epsilon}^{(1)}\left(\omega_{n}\right) \mathbf{E}_{n}(\mathbf{x}),
$$

where $\hat{\epsilon}^{(1)}\left(\omega_{n}\right)=1+4 \pi \hat{\chi}^{(1)}\left(\omega_{n}\right)$ introduces the frequency-dependent linear coefficient of dielectric permittivity, which in case of a gas can be represented by a scalar along with $\hat{\chi}^{(1)}\left(\omega_{n}\right)$, see (1.21). Separately for the real and imaginary parts:

$$
\begin{aligned}
& \operatorname{Re} \hat{\epsilon}^{(1)}\left(\omega_{n}\right)=1+4 \pi \operatorname{Re} \hat{\chi}^{(1)}\left(\omega_{n}\right), \\
& \operatorname{Im} \hat{\epsilon}^{(1)}\left(\omega_{n}\right)=4 \pi \operatorname{Im} \hat{\chi}^{(1)}\left(\omega_{n}\right) .
\end{aligned}
$$

Substituting (1.33)-(1.39) into (1.36) and taking into account (1.43), we obtain 
the wave equation for each frequency component of the field:

$$
\partial_{t}^{2} \mathbf{E}_{n}-\frac{c^{2}}{\hat{\epsilon}^{(1)}\left(\omega_{n}\right)} \triangle \mathbf{E}_{n}=-\frac{4 \pi}{\hat{\epsilon}^{(1)}\left(\omega_{n}\right)} \partial_{t}^{2} \mathbf{P}_{n}^{N L}
$$

Consider two frequency components: initial(fundamental) wave, $\mathbf{E}_{1}(z, t)$, with frequency $\omega$ and wavenumber $k$ and $\mathbf{E}_{3}(z, t)$, of frequency $3 \omega$ with wavenumber $k_{3}$, both propagating as linearly polarized (LP) quasi-plane waves forward and backward in the $z$-direction:

$$
\begin{aligned}
& \mathbf{E}_{1}(z, t) \equiv \mathbf{E}_{1}=\mathbf{e}_{\perp}\left[A_{1}(z) e^{\mathbf{i}(k z-\omega t)}+A_{1}^{*}(z) e^{-\mathbf{i}(k z-\omega t)}\right] \\
& \mathbf{E}_{3}(z, t) \equiv \mathbf{E}_{3}=\mathbf{e}_{\perp}\left[A_{3}(z) e^{i\left(k_{3} z-3 \omega t\right)}+A_{3}^{*}(z) e^{-i\left(k_{3} z-3 \omega t\right)}\right],
\end{aligned}
$$

where the complex amplitudes, $A_{1}(z)$ and $A_{3}(z)$, are taken as slowly varying functions of $z$ only, and where $\mathbf{e}_{\perp}$ denotes the direction perpendicular to $\mathbf{e}_{\mathbf{z}}$.

The derivatives of the field-components w.r.t. time are

$$
\begin{aligned}
\partial_{t}^{2} \mathbf{E}_{1}(z, t) & =-\omega^{2} \mathbf{E}_{1}(z, t), \\
\partial_{t}^{2} \mathbf{E}_{3}(z, t) & =-9 \omega^{2} \mathbf{E}_{3}(z, t),
\end{aligned}
$$

and their Laplacian is

$$
\begin{aligned}
\triangle \mathbf{E}_{1}(z, t) & =\mathbf{e}_{\perp}\left[\left(-k^{2} A_{1}(z)+\frac{d^{2} A_{1}(z)}{d z^{2}}+2 \mathrm{i} k \frac{d A_{1}(z)}{d z}\right) e^{\mathrm{i}(k z-\omega t)}+\right. \\
+ & \left.\left(-k^{2} A_{1}^{*}(z)+\frac{d^{2} A_{1}^{*}(z)}{d z^{2}}-2 \mathrm{i} k \frac{d A_{1}^{*}(z)}{d z}\right) e^{\mathrm{i}(k z-\omega t)}\right] \\
\triangle \mathbf{E}_{3}(z, t) & =\mathbf{e}_{\perp}\left[\left(-k_{3}^{2} A_{3}(z)+\frac{d^{2} A_{3}(z)}{d z^{2}}+2 \mathrm{i} k_{3} \frac{d A_{3}(z)}{d z}\right) e^{\mathrm{i}\left(k_{3} z-3 \omega t\right)}+\right. \\
+ & \left.\left(-k_{3}^{2} A_{3}^{*}(z)+\frac{d^{2} A_{3}^{*}(z)}{d z^{2}}-2 \mathrm{i} k_{3} \frac{d A_{3}^{*}(z)}{d z}\right) e^{\mathrm{i}\left(k_{3} z-3 \omega t\right)}\right]
\end{aligned}
$$

Before substituting the derivatives (1.48) and (1.49) into the wave equation (1.45), we also need to compute the nonlinear polarization associated with the three-photon 
processes. Following the expressions (1.26), (1.28) and (1.46), (1.47), we get

$$
\begin{aligned}
\mathbf{P}_{1}^{N L}(z, t) & =\mathbf{e}_{\perp}\left[P(z, \omega) e^{-i \omega t}+P^{*}(z, \omega) e^{i \omega t}\right]= \\
& =\mathbf{e}_{\perp}\left[\hat{\chi}^{(3)}(\omega)\left|A_{1}(z)\right|^{2}\left(A_{1}(z) e^{\mathbf{i}(k z-\omega t)}+A_{1}^{*}(z) e^{-i(k z-\omega t)}\right)\right], \\
\mathbf{P}_{3}^{N L}(z, t) & =\mathbf{e}_{\perp}\left[P(z, 3 \omega) e^{-3 i \omega t}+P^{*}(z, 3 \omega) e^{3 i \omega t}\right]= \\
& =\mathbf{e}_{\perp}\left[\hat{\chi}^{(3)}(3 \omega)\left(A_{1}^{3}(z) e^{3 i(k z-\omega t)}+A_{1}^{* 3}(z) e^{-3 i(k z-\omega t)}\right)\right],
\end{aligned}
$$

where we have denoted $\hat{\chi}^{(3)}(\omega)=\hat{\chi}_{1}^{(3)}(\omega)+\hat{\chi}_{2}^{(3)}(\omega)$ since in the case of LP wave $|\mathbf{E}(z, \omega)|^{2} \mathbf{E}(z, \omega)=(\mathbf{E}(z, \omega) \cdot \mathbf{E}(z, \omega)) \mathbf{E}^{*}(z, \omega)=\left|A_{1}(z)\right|^{2} A_{1}(z)$.

Taking into account the dispersion relations, which in the first approximation are:

$$
\begin{aligned}
\omega^{2} & =\frac{c^{2} k^{2}}{\hat{\epsilon}^{(1)}(\omega)} \equiv \frac{c^{2} k^{2}}{n_{0}^{2}(\omega)}, \\
(3 \omega)^{2} & =\frac{c^{2} k_{3}^{2}}{\hat{\epsilon}^{(1)}(3 \omega)} \equiv \frac{c^{2} k_{3}^{2}}{n_{0}^{2}(3 \omega)},
\end{aligned}
$$

where $n_{0}(\omega)=\sqrt{\hat{\epsilon}^{(1)}(\omega)}$ denotes the linear refractive index, we finally obtain the wave equations for each harmonic (together with the complex conjugate ones):

$$
\begin{aligned}
& \frac{d^{2} A_{1}(z)}{d z^{2}}+2 \mathrm{i} k \frac{d A_{1}(z)}{d z}=-\frac{4 \pi \omega^{2}}{c^{2}} \hat{\chi}^{(3)}(\omega)\left|A_{1}(z)\right|^{2} A_{1}(z), \\
& \frac{d^{2} A_{3}(z)}{d z^{2}} e^{\mathrm{i} k_{3} z}+2 \mathrm{i} k_{3} \frac{d A_{3}(z)}{d z} e^{\mathrm{i} k_{3} z}=-\frac{36 \pi \omega^{2}}{c^{2}} \hat{\chi}^{(3)}(3 \omega) A_{1}^{3}(z) e^{3 \mathrm{i} k z} .
\end{aligned}
$$

In the Slowly Varying Envelope Approximation (SVEA) [24], we can neglect the second derivative in $z$ w.r.t. the alteration of the first derivative on the wavelength scale:

$$
\left|\frac{d^{2} A_{1,3}}{d z^{2}}\right| \ll\left|k \frac{d A_{1,3}}{d z}\right|
$$

We also use here the constant-pump approximation: $\left|A_{3}\right| \ll\left|A_{1}\right|$, which allows to set $\left|A_{1}(z)\right|^{2}=\left|A_{0}\right|^{2}=2 \pi I_{1} / n_{0} c$, where $I_{1}$ is an intensity, associated with the electric 
field (1.46) of the laser pulse of frequency $\omega[24]$ :

$$
I_{1}=\frac{c n(\omega)\left|A_{1}\right|^{2}}{2 \pi}
$$

Now, taking into account the corresponding linear dispersion relation (1.52), we obtain for the fundamental harmonic, the following propagation equation

$$
\frac{d A_{1}(z)}{d z}=i \frac{4 \pi^{2} \omega \hat{\chi}^{(3)}(\omega)}{c^{2} n_{0}^{2}(\omega)} I_{1} A_{1}(z)
$$

solution of which is

$$
A_{1}(z)=A_{0} \exp \left(i \frac{4 \pi^{2} \omega \hat{\chi}^{(3)}(\omega)}{c^{2} n_{0}^{2}(\omega)} I_{1} z\right) .
$$

Turning back to the electric field (1.46), we re-write the expression as

$$
\mathbf{E}_{1}=\mathbf{e}_{\perp}\left[A_{0} e^{i\left(k\left(\omega, I_{1}\right) z-\omega t\right)}+A_{0}^{*} e^{-i\left(k\left(\omega, I_{1}\right) z-\omega t\right)}\right]
$$

where the wave number $k\left(\omega, I_{1}\right)$ is given by

$$
k\left(\omega, I_{1}\right)=\frac{\omega}{c}\left(n_{0}(\omega)+4 \pi^{2} \frac{\hat{\chi}^{(3)}(\omega)}{c n_{0}^{2}(\omega)} I_{1}\right) .
$$

Here one can conclude that the refractive index, which is defined as the ratio of the speed of light in vacuum $c$, to the phase velocity of light in the medium, $\omega / k\left(\omega, I_{1}\right)$, reads as

$$
n(\omega) \approx n_{0}(\omega)+n_{2}(\omega) I_{1}
$$

where

$$
n_{2}(\omega)=4 \pi^{2} \frac{\hat{\chi}^{(3)}(\omega)}{c n_{0}^{2}(\omega)}
$$

and, although $I_{1}$ is large, the product $n_{2} I_{1}$ gives the small nonlinear correction to refractive index: $n_{0} \gg\left|n_{2}\right| I_{1}$. For typical estimation of the cubic susceptibility 
$\chi^{(3)} \approx 6 \times 10^{-24} \mathrm{~m}^{2} / \mathrm{V}^{2}[25]$, and for laser intensity $I_{1}=10^{14} \mathrm{~W} / \mathrm{cm}^{2}$, we obtain $n_{2} I_{1} \approx 10^{-3}$.

Notice, the expression (1.62), known as the optical Kerr effect, can be extended further in powers of $I_{1}$. Indeed, as we mentioned before, in case of a monatomic gas the series for $\widehat{\mathbf{P}}^{N L}$ contains the odd terms only: $\widehat{\mathbf{P}}^{N L}(\omega)=\sum_{m=1}^{\infty} \widehat{\mathbf{P}}^{(2 m+1)}(\omega)$, where

$$
\begin{gathered}
\widehat{P}_{i}^{(2 m+1)}(\omega)=D \int_{-\infty}^{\infty} \hat{\chi}_{i j k \ldots s}^{(2 m+1)}\left(\omega ; \omega_{1}, \omega_{2}, \ldots, \omega_{2 m+1}\right) \widehat{E}_{j}\left(\omega_{1}\right) \widehat{E}_{k}\left(\omega_{2}\right) \ldots \widehat{E}_{s}\left(\omega_{2 m+1}\right) \times \\
\times \delta\left(\omega_{1}+\omega_{2}+\cdots+\omega_{2 m+1}-\omega\right) d \omega_{1} d \omega_{2} \ldots d \omega_{2 m+1}
\end{gathered}
$$

and the factor represented by the Dirac delta function, $\delta\left(\omega_{1}+\omega_{2}+\cdots+\omega_{2 m+1}-\omega\right)$, cares for compliance of the energy conservation law, while $D$ is the degeneracy factor counting the number of distinct permutations of the frequencies $\omega_{1}, \omega_{2} \ldots \omega_{2 m+1}$ giving in total $\omega$.

Now, substituting in the RHS of the wave equation (1.45), written for the frequency of the falling pulse $\omega$, the series for nonlinear polarization vector $\widehat{\mathbf{P}}^{N L}(\omega)$, we obtain in the bounds of the perturbative theory (see (1.58)-(1.61)) the following expansion for the refractive index:

$$
n(\omega)=n_{0}(\omega)+n_{2} I_{1}+n_{4} I_{1}^{2}+\cdots+n_{2 m} I_{1}^{m}+\ldots,
$$

where $n_{2 m} \propto \chi^{(2 m+1)}(\omega)$ and each succeeding term is smaller than the preceding: $\left|n_{2 m-2}\right| \gg\left|n_{2 m}\right| I_{1}(m \geq 1)$, as we still assume that $\left|\mathbf{P}^{(2 m-1)}\right| \gg\left|\mathbf{P}^{(2 m+1)}\right|$.

Back to the equation (1.55), we establish the intensity $I_{3}$ of the third harmonic. Rewriting the equation in the SVEA (1.56), and assuming that the principal harmonic changes with $z$ slowly, we approximate $A_{1}(z)$ by a constant $A_{1}$, and taking into account the dispersion relation (1.53), we get

$$
\frac{d A_{3}(z)}{d z}=i \frac{6 \pi \omega \hat{\chi}^{(3)}(3 \omega) A_{1}^{3}}{c n_{0}(3 \omega)} e^{i\left(3 k-k_{3}\right) z}
$$




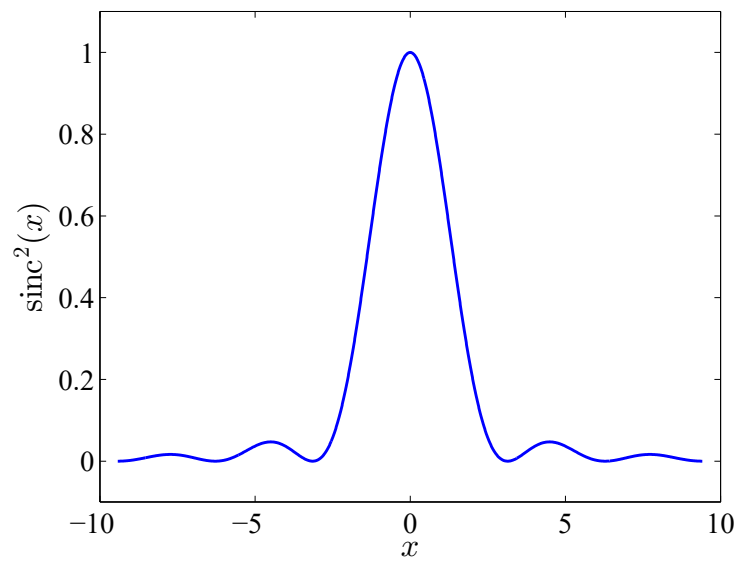

Figure 1.4: The phase mismatch factor from (1.67), $x:=\Delta k L / 2, \Delta k:=3 k-k_{3}$.

Thus, after propagation of the intense initial pulse in a gas from $z_{0}=0$ to $z=L$ :

$$
A_{3}(L)=\frac{6 \pi \omega \hat{\chi}^{(3)}(3 \omega) A_{1}^{3}}{c n_{0}(3 \omega)} \frac{e^{i\left(3 k-k_{3}\right) L}-1}{3 k-k_{3}},
$$

and finally, the intensity of the third harmonics $I_{3}=c n_{0}(3 \omega)\left|A_{3}\right|^{2} / 2 \pi$, is

$$
I_{3}(L)=\frac{144 \pi^{4} \omega^{2}\left[\hat{\chi}^{(3)}(3 \omega)\right]^{2} I_{1}^{3} L^{2}}{c^{4} n_{0}(3 \omega) n_{0}^{3}(\omega)} \times \frac{\sin ^{2}\left(\frac{3 k-k_{3}}{2} L\right)}{\left(\frac{3 k-k_{3}}{2} L\right)^{2}}
$$

As we can see, the expression for the intensity of the third harmonic includes the so called [24] phase mismatching factor, $\operatorname{sinc}^{2}(x)$, where $\operatorname{sinc}(x)=\sin (x) / x$, see Fig.1.4.

Hence, the efficiency of the THG is maximal if

$$
\Delta k=3 k-k_{3}=0
$$

and decreases rapidly with the increase of the parameter $\Delta k L / 2$. Note that in the quantum mechanical sense, the condition (1.68) expresses the law of conservation of momentum in the process where three photons of frequency $\omega$ and momentum $\hbar \mathbf{k}$ each completely transfer their total momentum to the new photon of frequency $3 \omega$ and 


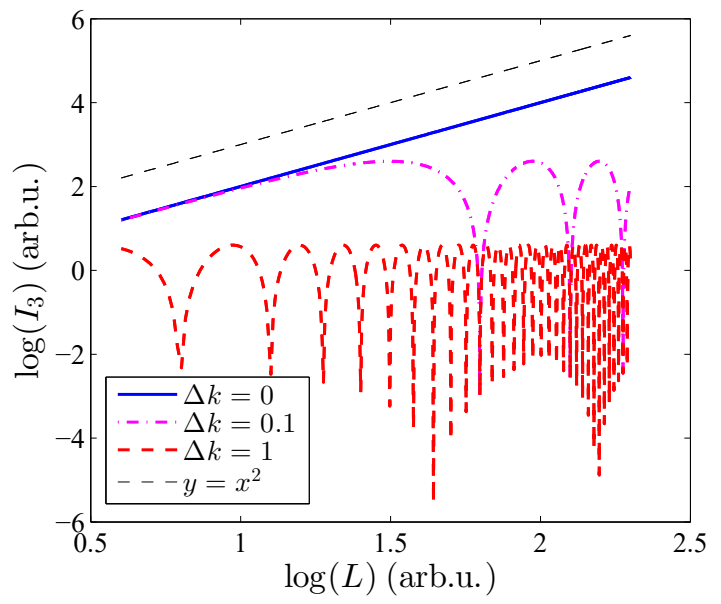

FIGURE 1.5: Intensity of the third harmonic, $I_{3}$, as a function of the propagating length, $L$, for different values of the wavevector mismatch $\Delta k$.

momentum $\hbar \mathbf{k}_{3}: \hbar(\mathbf{k}+\mathbf{k}+\mathbf{k})=\hbar \mathbf{k}_{3}$. Thus, the conservation laws for energy and momentum provide the most efficient (coherent in sense of the phases of the fundamental and third harmonics) energy transfer from the principal harmonic to the third one (as well as to any others odd harmonics in case of a monatomic gas). It is clear that in the absence of dispersion, the condition (1.68) is satisfied automatically. However, if we re-write it in terms of the refractive indices, we get $\frac{3 \omega}{c}\left(n_{0}(\omega)-n_{0}(3 \omega)\right)=0$, which cannot be satisfied in the case of medium with normal dispersion, i.e. when $n_{0}(\omega)$ is a monotonically increasing function of $\omega$. As an example, Fig. 1.5 shows how the non-zero $\Delta k$ affects the intensity of the third harmonic: during the propagation of the waves their different dispersion laws result in mismatch between the phases of the third harmonic and its driving polarization, that leads to a reduction of the pump efficiency [24]. Moreover, in some space conditions energy can flow back from $3 \omega$-harmonic to the fundamental one, see the oscillations in Fig. 1.5.

Recall, that in all the previous examples, we have considered the infinite quasiplane wave (weakly dependent on $z$-coordinate), while in nonlinear optics the case of the compact beam is more often reasonable and realistic [24], [94]. Also in the expression (1.47) we have neglected the possible amplitude dependence on the trans- 
verse coordinate. Now, following the presentation given in [24], we write down the paraxial approximation in a more general form of the nonplane waves satisfying the wave equation (1.45):

$$
\begin{aligned}
& \mathbf{E}_{n}(\mathbf{x}, \mathbf{t})=\mathbf{A}_{n}(\mathbf{x}) e^{\mathbf{i}\left(k_{n} z-\omega_{n} t\right)}+\mathbf{A}_{n}^{*}(\mathbf{x}) e^{\mathbf{i}\left(k_{n} z-\omega_{n} t\right)} \\
& \mathbf{P}_{n}(\mathbf{x}, \mathbf{t})=\boldsymbol{\Pi}_{n}(\mathbf{x}) e^{\mathbf{i}\left(k_{n}^{\prime} z-\omega_{n} t\right)}+\boldsymbol{\Pi}_{n}^{*}(\mathbf{x}) e^{-\mathbf{i}\left(k_{n}^{\prime} z-\omega_{n} t\right)},
\end{aligned}
$$

where $\mathbf{A}_{n}$ and $\boldsymbol{\Pi}_{n}$ are spatially varying amplitudes of the harmonics $\left(k_{n}, \omega_{n}\right)$ and $\left(k_{n}^{\prime}, \omega_{n}\right)$ respectively, and it is allowed the possibility of a wavevector mismatch, as in general $k_{n} \neq k_{n}^{\prime}$. Substituting (1.69) into the wave equation (1.45), taking into account the dispersion relation (1.52) and applying the SVEA just as in (1.56), we finally obtain the paraxial wave equation (the Helmholtz equation):

$$
2 i k_{n} \partial_{z} \mathbf{A}_{n}+\triangle_{\perp} \mathbf{A}_{n}=-\frac{4 \pi \omega_{n}^{2}}{c^{2}} \mathbf{\Pi}_{n} e^{i \Delta k z}
$$

where $\Delta k=k_{n}^{\prime}-k_{n}$ and $\Delta_{\perp}$ denotes the transverse to $z$-direction Laplacian operator.

In vacuum, i.e. in the absence of the driving polarization, the solution of (1.70) in case of cylindrical symmetry for $\mathbf{A}(r, z)=A(r, z) \mathbf{e}_{\perp}$ is known as the Gaussian beam with the waist (focus) $w_{0}$ :

$$
A(r, z)=\frac{\mathcal{A}}{1+i \zeta} e^{-r^{2} / w_{0}^{2}(1+i \zeta)}
$$

where $\zeta=2 z / b$ is a dimensionless longitudinal coordinate, expressed in terms of the confocal parameter $b=k w_{0}^{2}$. Then the intensity distribution reads as

$$
I(r, z):=\frac{c n_{0}|A(r, z)|^{2}}{2 \pi}=I_{0}\left(\frac{w_{0}}{w(z)}\right)^{2} e^{-2 r^{2} / w^{2}(z)}
$$

where $I_{0}=I(0,0)=c n_{0}|\mathcal{A}|^{2} / 2 \pi$ and the function $w(z)=w_{0} \sqrt{1+\zeta^{2}(z)}$ corresponds (at given $z$ ) to the radius $\rho$, where the field amplitude distribution decreases in $e$ 

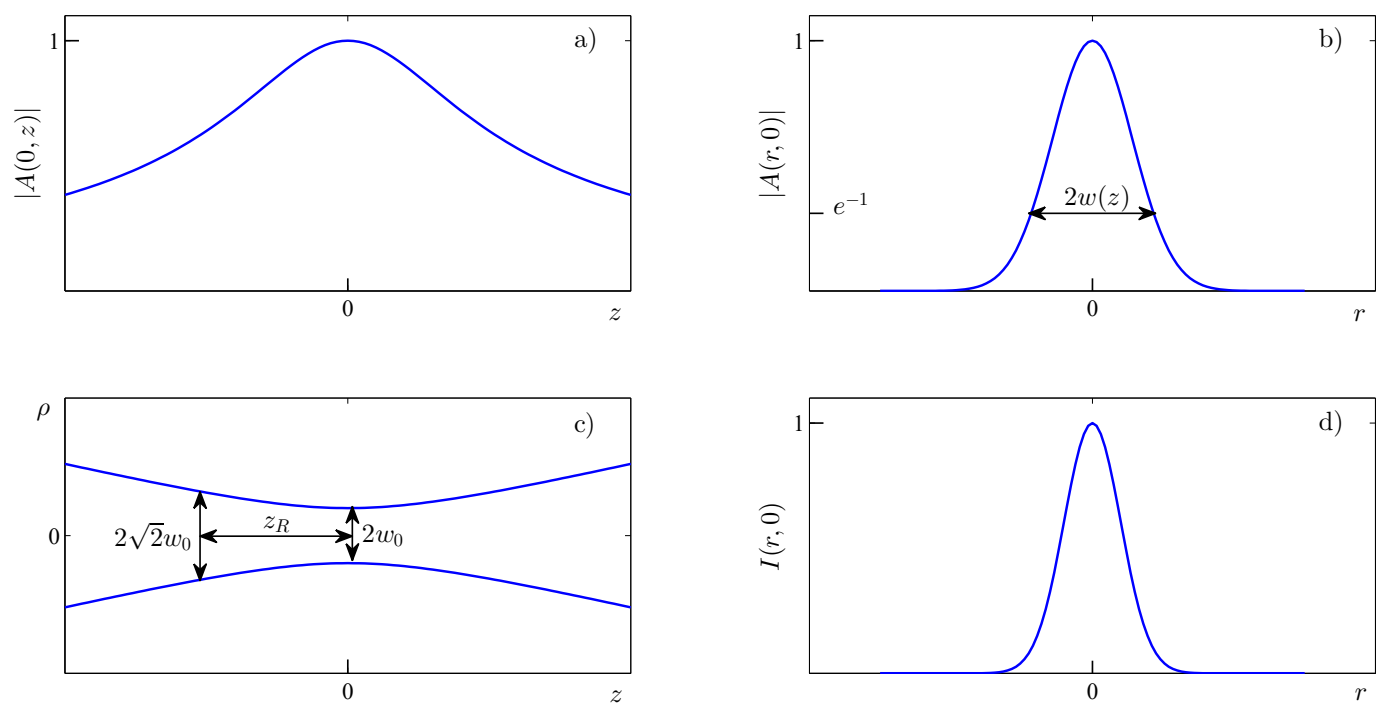

FiguRE 1.6: Field amplitude distribution of a Gaussian beam (a) at $r=0$; (b) at $z=0$; (c) the beam "effective" radius $\rho=w(z)$, beam waist $w_{0}$, Rayleigh length $z_{R}$; (d) intensity distribution at $z=0$.(arb.units)

times. At the Rayleigh radius $z_{R}=b / 2$, the beam radius $w(z)=\sqrt{2} w_{0}$, see Fig. 1.6.

Consider now the generation of the $q^{\text {th }}$ harmonic, using the focused Gaussian beam (1.71) as the fundamental wave $(n=1)$, with $A_{1} \equiv A, \mathcal{A}_{1} \equiv \mathcal{A}, k_{1} \equiv k, \omega_{1} \equiv \omega[24]$ and accepting $\Pi_{q}=\chi^{(q)} A_{1}^{q}$ (see for a reason (1.51) and (1.64)). In the constant-pump approximation (implying that $\mathcal{A}_{1}$ remains constant) the amplitude $A_{q}$ of the $\omega_{q}=q \omega$ frequency component can be sought in the form of the trial solution:

$$
A_{q}(r, z)=\frac{\mathcal{A}_{q}(z)}{1+\mathrm{i} \zeta} e^{-q r^{2} / w_{0}^{2}(1+\mathrm{i} \zeta)}
$$

After substituting (1.73) into (1.70) the equation for the amplitude occurs similar to (1.66), and can be integrated directly to obtain:

$$
\mathcal{A}_{q}(z)=\frac{2 \pi i q \omega}{n_{0}(q \omega) c} \chi^{(q)} \mathcal{A}_{1}^{q} J_{q}\left(\Delta k, z_{0}, z\right)
$$

where $\Delta k:=q k_{1}-k_{q}$ and the factor describing phase matching is 


$$
J_{q}\left(\Delta k, z_{0}, z\right)=\int_{z_{0}}^{z} \frac{e^{\mathrm{i} \Delta k z^{\prime}}}{\left(1+2 \mathrm{i} z^{\prime} / b\right)^{q-1}} d z^{\prime}
$$

i.e. the nonlinear medium is contained in the range $\left[z_{0}, z\right]$. Denote $L=z-z_{0}$, then in the plane-wave limit, when the typical scale of focusing $b \gg\left|z_{0}\right|,|z|$, the integration of $(1.75)$ gives $J_{q}\left(\Delta k, z_{0}, z\right)=L \operatorname{sinc}\left(\frac{\Delta k L}{2}\right) e^{i \frac{\Delta k\left(z+z_{0}\right)}{2}}$, which in case $q=3$ results in the expression for intensity, consistent with (1.67). However, in the opposite limit of the tightly-focusing beam $\left(b \ll\left|z_{0}\right|,|z|\right)$, the solution has an interesting feature [24]. Indeed, in such approximation the integrand

$$
J_{q}\left(\Delta k, z_{0}, z\right)=J_{q}(\Delta k)=\int_{-\infty}^{\infty} \frac{e^{\mathrm{i} \Delta k z^{\prime}}}{\left(1+2 \mathrm{i} z^{\prime} / b\right)^{q-1}} d z^{\prime}
$$

is not a holomorphic function because of a pole of order $q-1$ lying in the upper semiplane at $z^{\prime}:=b / 2$. Thus, applying Cauchy's integral formula we obtain $J_{q}(\Delta k)=$ 0 for $\Delta k \leqslant 0$, while for $\Delta k>0$ :

$$
\begin{aligned}
& J_{q}(\Delta k)=2 \pi i\left(\frac{b}{2 i}\right)^{q-1} \operatorname{Res}\left(\frac{e^{i \Delta k z}}{(z-i b / 2)^{q-1}}, \frac{i b}{2}\right)= \\
& =2 \pi i\left(\frac{b}{2 i}\right)^{q-1} \frac{1}{(q-2) !} \lim _{z \rightarrow i b / 2} \frac{d^{q-2}}{d z^{q-2}} e^{i \Delta k z}=\frac{2 \pi}{(q-2) !} \frac{b}{2}\left(\frac{b \Delta k}{2}\right)^{q-2} e^{-b \Delta k / 2} .
\end{aligned}
$$

The main difference with the case of a plane wave is that the maximal intensity of the $q^{\text {th }}$ harmonic falls on $\Delta k=2(q-2) / b$, while when $\Delta k=0$, we get $J_{q}=0$. The effect is referred to as Gouy phase shift that any light beam experiences in passing through the focus [24].

Summarizing Section 1.3.1, the important feature of the harmonics generation in perturbative regime in the isotropic medium is that their intensities rapidly decrease with the (odd) number of harmonic. It causes both a small magnitude of nonlinear susceptibility coefficient $\chi^{(3)} \approx 10^{-24} \mathrm{~m}^{2} \mathrm{~V}^{-2}[25]$ and difficulties in achieving of phase matching. 


\subsubsection{Self-Action Effects}

In the perturbative regime, we deal with nonlinear effects that gradually occur during the light propagation in a medium. If the refractive index of a gas depends on the intensity of the propagating pulse as in (1.62), so-called a Kerr medium, selfaction effects such as self-focusing, self-trapping and laser beam breakup can take place under certain conditions. The inception of the plane wave instability in the Kerr medium can be demonstrated on the basis of the paraxial equation (1.70), see e.g. [70]. Introduce a small perturbation of the solution (1.59), depending on $z$ and perpendicular coordinates $\mathbf{x}_{\perp}=(x, y)$, so that

$$
A_{1}\left(\mathbf{x}_{\perp}, z\right)=\left(A_{0}+\delta A\left(\mathbf{x}_{\perp}, z\right)\right) \exp \left(i \frac{6 \pi \omega \hat{\chi}^{(3)}(\omega)}{c n_{0}(\omega)}\left|A_{0}\right|^{2} z\right)
$$

then using (1.50), we get for $\delta A\left(\mathbf{x}_{\perp}, z\right)$ a linear Partial Differential Equation (PDE)

$$
\begin{aligned}
2 \mathrm{i} k \frac{\left.\partial \delta A\left(\mathbf{x}_{\perp}, z\right)\right)}{\partial z}+ & \left.\triangle_{\perp} \delta A\left(\mathbf{x}_{\perp}, z\right)\right)= \\
& \left.\left.=\frac{12 \pi \omega^{2} \chi^{(3)}(\omega)}{c^{2}}\left(\left|A_{0}\right|^{2} \delta A\left(\mathbf{x}_{\perp}, z\right)\right)+A_{0}^{2} \delta A^{*}\left(\mathbf{x}_{\perp}, z\right)\right)\right) .
\end{aligned}
$$

Let $\delta A\left(\mathbf{x}_{\perp}, z\right):=a e^{\mathbf{i} \mathbf{q} \cdot \mathbf{x}_{\perp}+\mathbf{i} \gamma z}+b e^{-\mathbf{i} \mathbf{q} \cdot \mathbf{x}_{\perp}-\mathbf{i} \gamma z}$, where $a, b \in \mathbb{C}, \mathbf{q} \in(x, y)$ and $\gamma$ determines the small perturbation evolution during its propagation along the $z$-axis. Substituting $\delta A$ in that form into (1.77) we obtain the homogeneous linear system

for $a$ and $b$. We set equal the determinant to 0 and find $\gamma= \pm \frac{q}{2 k} \sqrt{q^{2}-4 k Q}$, where $q=|\mathbf{q}|, Q=6 \pi \chi^{(3)} \omega\left|A_{0}\right|^{2} / c n_{0}$. Thus, in the case $q^{2}<4 k Q$ the coefficient $\gamma$ is imaginary, and the solution becomes exponentially increasing (in linear approximation). One of the manifestations of such instability is the so-called self-focusing [4].

Following [24] we demonstrate that the Kerr medium with diffraction acts as a converging lens with a focal length $z_{\text {sf }}$, see Fig 1.7 (Top). To that end, we will use the equivalent model with the same value of $z_{\mathrm{sf}}$, but with different paths of the rays, see Fig 1.7 (Bottom). To begin with, consider the propagation of the laser beam in the 


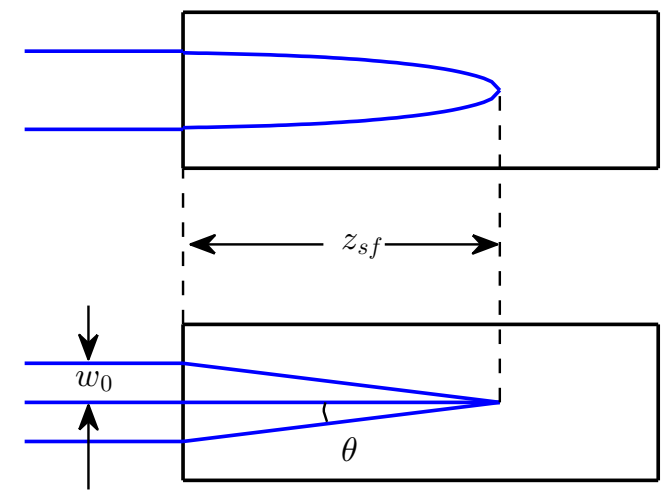

Figure 1.7: Self-focusing it the Kerr medium (Top) and equivalent model used to evaluate $z_{\text {sf }}$ (Bottom).

Kerr medium ignoring diffraction effects and assuming $n_{2}>0$. Employing Fermat's principle, one can set equal the optical path lengths for the central and the marginal rays traveling from a wavefront at the angle $\theta_{\text {sf }}$ between them [24]:

$$
\left(n_{0}+n_{2} I\right) z_{\mathrm{sf}}=n_{0} z_{\mathrm{sf}} / \cos \theta_{\mathrm{sf}}
$$

where $z_{\text {sf }}$ is the characteristic geometrical self-focusing distance. Since the nonlinearity is weak $\left(n_{2} I \ll n_{0}\right)$, the self-focusing angle is expected to be small, so up to the second order $1 / \cos \theta_{\mathrm{sf}} \approx 1+\theta_{\mathrm{sf}}^{2} / 2$, and hence

$$
\theta_{\mathrm{sf}} \approx \sqrt{2 n_{2} I / n_{0}}
$$

Now we take into account the beam divergence. Recall that in case of far-field diffraction (known as Fraunhofer diffraction) of the beam on a round hole of diameter $d$, the angular intensity $d I$ in solid angle $d \Omega$ is [68]

$$
\frac{d I}{d \Omega}=I_{0} \frac{J_{1}^{2}\left(\pi \theta d / \lambda_{0}\right)}{\pi \theta^{2}}
$$


where $\lambda_{0}$ is a vacuum wavelength, $I_{0}$ is the total intensity of the falling parallel beam, $\theta$ is an angle between the normal to the hole and the scattered ray, and $J_{1}$ is the Bessel function of the first kind and order one. To evaluate the diffraction angle we can take the first zero of the expression (1.80), i.e. the second zero of $J_{1}(x)$, which is attained at $x=3.817$ (recall although $J_{1}(0)=0$, but $\lim _{x \rightarrow 0} J_{1}(x) / x=0.5$ ). Then in the medium with refraction index $n_{0}$, the required angle is

$$
\theta_{\text {diff }} \approx \frac{3.817 \lambda_{0}}{\pi n_{0} d} \approx \frac{1.22 \lambda_{0}}{n_{0} d}
$$

If in linear approximation $\theta_{\mathrm{sf}}>\theta_{\text {diff }}$, then we can estimate the resulting focusing angle $\theta_{\text {eff }}$ from the expression for the optical path difference

$$
\frac{n_{0} \widetilde{z}_{\mathrm{sf}}}{\cos \theta_{\mathrm{sf}}}-\frac{n_{0} \widetilde{z}_{\mathrm{sf}}}{\cos \theta_{\mathrm{eff}}}=\frac{n_{0} \widetilde{z}_{\mathrm{sf}}}{\cos \theta_{\mathrm{diff}}}-n_{0} \widetilde{z}_{\mathrm{sf}}
$$

where $\widetilde{z}_{\mathrm{sf}}$ is a updated focusing length. From here one gets approximately $\theta_{\text {eff }}=$ $\sqrt{\theta_{\mathrm{sf}}^{2}-\theta_{\text {diff }}^{2}}$ and for the beam with initial radius $w_{0}$ (so that $d=2 w_{0}$ ), we can reevaluate $\widetilde{z}_{\mathrm{sf}}$ as

$$
\widetilde{z}_{\mathrm{sf}}=\frac{w_{0}}{\theta_{\mathrm{eff}}}=\frac{w_{0}}{\sqrt{\theta_{\mathrm{sf}}^{2}-\theta_{\mathrm{dif}}^{2}}}=\frac{n_{0} w_{0}^{2}}{0.61 \lambda_{0}} \frac{1}{\sqrt{P / P_{\mathrm{cr}}-1}},
$$

where the beam power $P=\pi w_{0}^{2} I$. Thus, if $P$ exceeds a critical value

$$
P_{\text {cr }}=\frac{\pi(0.61)^{2} \lambda_{0}^{2}}{2 n_{0} n_{2}}
$$

the self-focusing becomes possible in distance $\widetilde{z}_{\mathrm{sf}}$.

Recall that in the linear theory, any finitely cross-section beam diverges due to diffraction. As we just have seen, the nonlinear approach reveals a new phenomenon, which can qualitatively change the result of the interaction. Indeed, in the special case $\theta_{\text {sf }}=\theta_{\text {diff }}$, i.e. when diffraction effects are exactly balanced by the self-focusing, an interesting phenomena, refer to as self-trapping of light, can be observed. Setting 
equal (1.79) and (1.81) one obtains for the intensity of the pulse:

$$
I=\frac{(0.61)^{2} \lambda_{0}^{2}}{2 n_{2} n_{0} w_{0}^{2}}
$$

Thus, (i) the power contained in the beam does not depend on its diameter, and (ii) this power equals to the critical value $P_{\mathrm{cr}},[24]$. Due to total internal reflection at the boundary between the region with refractive index $n_{0}+n_{2} I$ (where the beam passes and changes the medium properties) and $n_{0}$ (external region, where the medium was not subject to the laser pulse), the laser beam remains trapped as the filament.

As soon as the power $P$ exceeds the critical value $P_{\mathrm{cr}}$, the laser beam can break into multiple filaments, each with a power equals $P_{\mathrm{cr}}$. A more detailed description of the phenomena is presented in [22], [24], [70]. Namely, employing the steady-state paraxial wave equation (1.70), we can consider the self-trapping of light in the case of a continuous-wave laser beam. As we study the fundamental harmonic only, we set $k^{\prime}=$ $k$ in (1.69) or $\Delta k=0$. Then in case of LP field $\mathbf{A}_{1}=A(x, z) \mathbf{e}_{\mathbf{x}}$, while the polarization according to $(1.28)$ is $\boldsymbol{\Pi}_{1}=\left[\left(\chi_{1}^{(3)}(\omega)+\chi_{2}^{(3)}(\omega)\right)|A|^{2} A\right] \mathbf{e}_{\mathbf{x}} \equiv\left[\chi^{(3)}(\omega)|A|^{2} A\right] \mathbf{e}_{\mathbf{x}}$. Hence we obtain the following PDE:

$$
i \frac{\partial A(x, z)}{\partial z}+\frac{1}{2 k} \frac{\partial^{2} A(x, z)}{\partial x^{2}}+\frac{2 \pi \omega \chi^{(3)}(\omega)}{c n_{0}}|A(x, z)|^{2} A(x, z)=0, \quad(x, z) \in \Omega
$$

in the form usually referred as Nonlinear Schrödinger Equation (NLSE), see [92,94, 114]. In addition, some analytical elements are given in Appendix B. As shown below, (1.85) possesses an envelope solitons. The domain of definition of the equation is $\Omega=\left\{(x, z): x \in \mathbb{R}, z \in \mathbb{R}_{+}\right\}$with a stationary source at $x=0, z=0: A_{0}:=A(0,0)$, and finite $|A(x, z)|_{x \rightarrow \pm \infty}$. It prompts to seek a solution in the form

$$
A(x, z)=A_{0} f(x) e^{\mathrm{i} \kappa(\omega) z}
$$


where $f(x)$ is a real-valued function with conditions $f(0)=1$ and $\lim _{x \rightarrow \pm \infty} f(x)$ finite. The factor $\kappa(\omega)$ is a correction to the wavenumber $k$ due to nonlinearity, similar to the second term in (1.61). From the symmetry of the problem in $x$, we can conclude that $f(x)=f(-x)$ or $f^{\prime}(0)=0$. Now substituting (1.86) into (1.85) and standing for $x_{0}^{-2}=2 \pi k \omega \hat{\chi}^{(3)}(\omega)\left|A_{0}\right|^{2} / c n_{0}=2 \pi \omega^{2} \hat{\chi}^{(3)}(\omega)\left|A_{0}\right|^{2} / c^{2}$, we obtain that $f$ satisfies the following $2^{\text {nd }}$ order ODE:

$$
f^{\prime \prime}(x)=f(x)\left(2 k \kappa(\omega)-2 x_{0}^{-2} f^{2}(x)\right)
$$

allowing the order reduction by employing a standard substitution $g=f^{\prime}$. After multiplying both sides by $f^{\prime}$ and integrating under conditions $g(1)=g(f(0))=$ $f^{\prime}(0)=0$, we obtain

$$
g^{2}(f)=x_{0}^{-2} f^{2}\left(2 x_{0}^{2} k \kappa(\omega)-f^{2}(x)\right)
$$

that immediately gives the nonlinear correction to wavenumber $\kappa(\omega)=\left(2 x_{0}^{2} k\right)^{-1}=$ $\pi \omega \hat{\chi}^{(3)}(\omega)\left|A_{0}\right|^{2} / c n_{0}$. The resulting equation in $f$ is

$$
f^{\prime}(x)=x_{0}^{-1} f(x) \sqrt{1-f^{2}(x)}, \quad f(0)=1,
$$

which can be easily integrated, and gives $f(x)=\operatorname{sech}\left(x / x_{0}\right)$, so that the solution of (1.85) is then

$$
A(x, z)=A_{0} \operatorname{sech}\left(x / x_{0}\right) e^{i \kappa(\omega) z} .
$$

Thus, $x_{0}$ gives the characteristic width of the beam. In particular, the more power the beam transfers, the narrower becomes its cross-section. In reality, from (1.87) we cannot conclude about the "cross-section", because if we add a $y$-coordinate, the problem cannot be solved analytically. However, numerical results confirm such inference. Moreover, the numerical simulations revealed the multifocus structure of the self-focusing process [106]. If the power of the beam is strong enough, during its 
propagation several focuses can be formed on the axis. Finally, if the power of the beam is much higher than the critical value (estimated $P>100 P_{c r}$ ) as a result of instability, the laser beam breakup may occur $[22,24]$.

In the end of the section, we make an important remark concerning a physical parameter, which tentatively "distinguishes" perturbative regime from nonperturbative [24]. Such a parameter is the characteristic atomic electric field strength $E_{\text {at }}=e / a_{0}^{2}$, where $e$ is the electron charge and $a_{0}$ is the Bohr radius of the hydrogen atom, so, we can evaluate $E_{\text {at }}=5.14 \times 10^{9} \mathrm{~V} / \mathrm{cm}$, which corresponds to an intensity of $I_{\text {at }}=3.49 \times 10^{16} \mathrm{~W} / \mathrm{cm}^{2}$. Accordingly, we can expect that nonlinear optics phenomena can occur if the electric field strength of the light becomes somewhat comparable with $E_{\text {at }}$, in practice even 1-2 order of magnitude smaller. When the laser field remains weaker than atomic one, we are mostly dealing with bound electron states and far from the resonances where the perturbation theory is valid. However, if the laser field strength is the same order or even exceeds $E_{\text {at }}$, the medium is subject to strong ionization, and perturbation theory cannot be used because the series (1.8) is not even valid for $\mathbf{P}(\mathbf{x}, t)$. 


\subsection{Nonperturbative Processes}

In this section, we will provide an introduction to two nonperturbative nonlinear optics phenomena, whose mathematical modeling is discussed in the present thesis. High harmonic generation (HHG) induced by laser beam in gases was discovered in late 1980s $[45,88]$ and has been extensively studied ever since then. The process allows to enter the world of attophysics, the branch of science studying the interatomic electrons dynamics, the time scale of which is the attosecond, 1 asec $=10^{-18} \mathrm{~s}$.

Another nonperturbative nonlinear phenomenon is the laser filament propagation in a gas [28], the travelling of laser pulses over large distances as a narrow "bullet". Although self-focusing, being one of the clues to understanding of filamentation, can be grasped even in perturbative regime, the phenomenon occurs only in essentially nonperturbative regime and hence requires an appropriate model.

\subsubsection{High Harmonic Generation}

High harmonics generation is a multiphoton process due to interaction of an intense short laser pulse with an atom or a molecule. To observe this phenomena, the incident laser frequency $\omega_{0}$ must be much smaller (in practice 10 or more) than the ionization potential $I_{p}$ of an atom (to avoid single-photon ionization). The irradiated atom then emits coherent radiation at frequencies $N \omega_{0}$, where $N \gg 10$ (in the experiments up to several tens or even hundreds). After the discovery of HHG, it has been established that the harmonic spectrum of the process has a universal form as illustrates Fig.1.8: (i) it falls off for the low order harmonics, then (ii) it exhibits a plateau and (iii) ends up sharply with cut-off $[24,73]$.

As discussed in the previous section, the emission low odd harmonics in gases (up to ionization threshold $\left.N_{\mathrm{P}} \approx I_{p} / \hbar \omega_{0}\right)$ results from the transitions between the bound electron states, thus, the rapid decrease in the harmonics intensity in that region is 


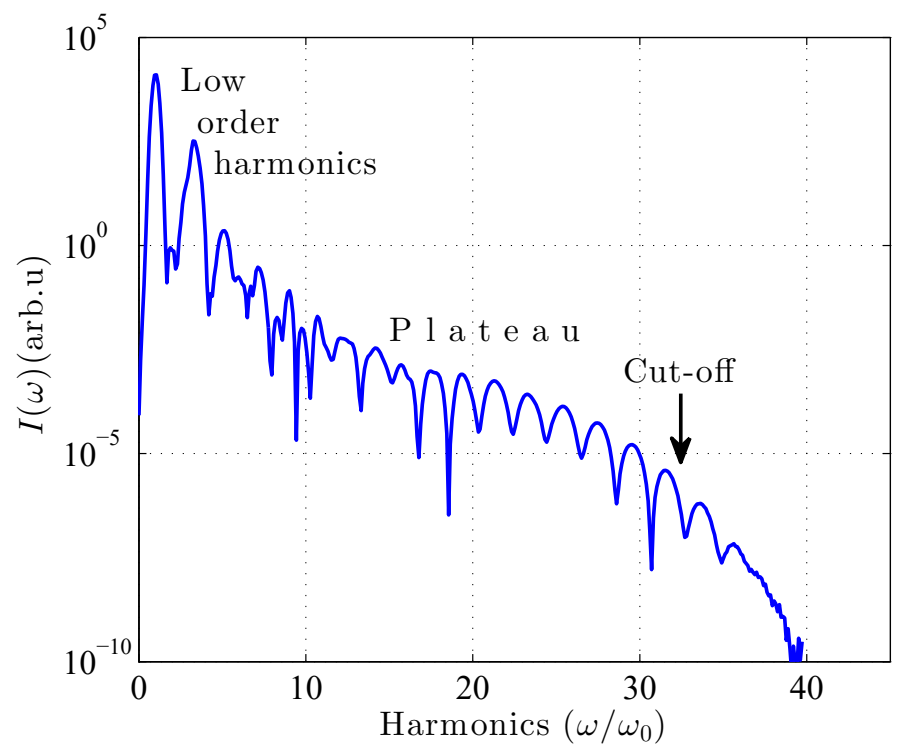

FIgURE 1.8: Shape of the typical HHG spectra.

an attribute of the perturbative regime. However, the plateau and cut-off on Fig.1.8 have essentially a nonperturbative nature. Harmonics in that region are formed due to participation of free electrons.

According to the semi-classical model by P.Corkum [35], HHG process maybe splitting in 3 stages (see for illustration Fig. 1.9): (i) tunneling of the electrons through the barrier formed by the atomic potential and laser field; (ii) acceleration of the electron in the laser field; (iii) recollision of the accelerated electrons with the parent nucleus/ion, which leads to the recombination and hence emission of high harmonics $[35,73]$. The semi-classical theory treats the stages one by one, and separately. It starts with computation of the ionization rate for tunnel ionization applying Keldysh's theory [58] and Ammosov-Delone-Krainov (ADK) formula [1]. Then the analysis of the classical Newtonian trajectory of a free electron with zero initial velocity in the field of the laser light is carried out [35]. There are two principal questions in this approach: (i) under which conditions the electron trajectory can return to the parent nucleus? and (ii) whether the electron gains an appropriate kinetic energy to produce a particular harmonic at the time of return? 


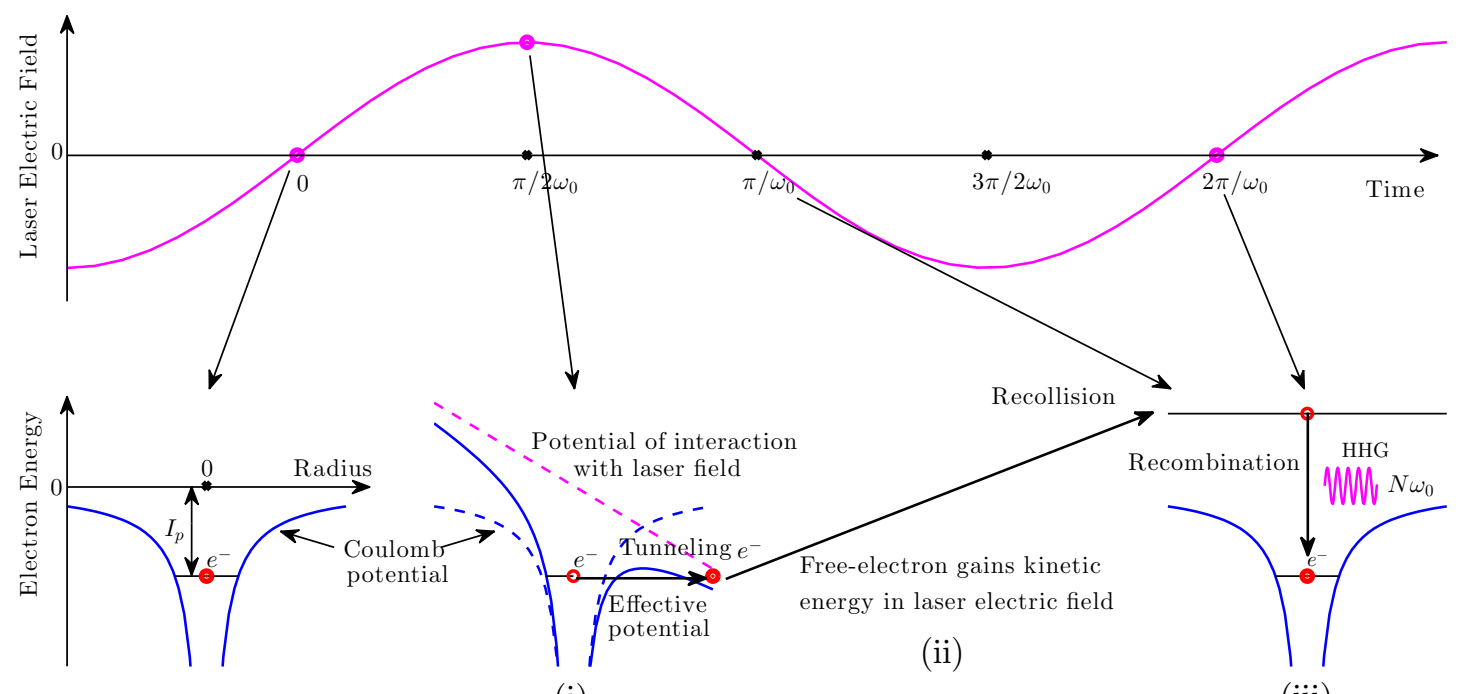

(i)

(iii)

Figure 1.9: Semi-classical model for HHG for hydrogen atom.

Note that the semi-classic theory is applied to dipole moments aligned along a linearly polarized (LP) laser field only [35]. It cannot so easily be extended in the case of circularly polarized (CP) wave. Moreover, the simple analysis of the classical trajectories shows that even in the case of a linearly polarized wave, the return to the vicinity of the parent nucleus is only possible for the electrons that became free at a certain wave phase intervals. Indeed, the solution obtained from Newton's second law in case of the field $E=E_{0} \sin \omega_{0} t$ and for the initial conditions at time of ionization $t_{0}$ (counted from the zero-phase of the wave): $v\left(t_{0}\right)=0, x\left(t_{0}\right)=0$, says

$$
\begin{aligned}
v(t)=\dot{x}(t) & =\frac{E_{0}}{\omega_{0}}\left(\cos \omega_{0} t-\cos \phi_{0}\right), \\
x(t) & =\frac{E_{0}}{\omega_{0}^{2}}\left(\sin \omega_{0} t-\sin \phi_{0}-\left(\omega_{0} t-\phi_{0}\right) \cos \phi_{0}\right)
\end{aligned}
$$

where $\phi_{0}=\omega_{0} t_{0}$ denotes the wave phase at ionization. The condition of the recollision at time $t_{R}$ is $x\left(t_{R}\right)=0$, from here with (1.89) we obtain

$$
\sin \phi_{R}-\sin \phi_{0}=\left(\phi_{R}-\phi_{0}\right) \cos \phi_{0}
$$


where $\phi_{R}=\omega_{0} t_{R}$. Introducing the phase difference $\Delta \phi=\phi_{R}-\phi_{0}$ we get

$$
\tan \phi_{0}=\frac{\Delta \phi-\sin \Delta \phi}{\cos \Delta \phi-1}
$$

As the RHS of the equality is obviously nonpositive, the first conclusion is: the recollision is possible only for those electrons that tunnel in the wave phase intervals: $\phi_{0} \in[\pi / 2, \pi] \cup[3 \pi / 2,2 \pi]$. The kinetic energy gained by such electrons is then

$$
K=\frac{\left(v\left(t_{R}\right)\right)^{2}}{2}=\frac{E_{0}^{2}}{2 \omega_{0}^{2}} \frac{(2(\cos \Delta \phi-1)+\Delta \phi \sin \Delta \phi)^{2}}{\left(2(1-\cos \Delta \phi)+\Delta \phi^{2}-2 \Delta \phi \sin \Delta \phi\right)} .
$$

We rewrite (1.92) in the form $K=U_{p} \Upsilon(\Delta \phi)$, where

$$
U_{p}=\frac{E_{0}^{2}}{4 \omega_{0}^{2}}
$$

the ponderomotive energy, the time-averaged kinetic energy, which a free electron gains in the electromagnetic field with frequency $\omega_{0}$ and amplitude $E_{0}$. The function

$$
\Upsilon(\Delta \phi)=\frac{2(2(\cos \Delta \phi-1)+\Delta \phi \sin \Delta \phi)^{2}}{\left(2(1-\cos \Delta \phi)+\Delta \phi^{2}-2 \Delta \phi \sin \Delta \phi\right)}
$$

reaches its maximum equals to 3.17 at $\Delta \phi=4.09$, which corresponds to $\phi_{0}=1.88$. Fig. 1.10 shows the graph $\Upsilon\left(\phi_{0}\right)$, computed using (1.91). It follows, that the maximal harmonic number that can be generated in the process is

$$
N_{\max } \approx\left(I_{p}+3.17 U_{p}\right) / \omega_{0}
$$

which is in very good agreement with the empirical formula obtained in the numerical simulations [64] even earlier the semi-classical theory was developed.

Note that according to the solution (1.91) the electron can return to the parent ion several times before it recombines there. These returns must be periodic, depending 
on the frequency of the laser radiation $\omega_{0}$. Figs. $\left.\left.1.11 \mathrm{a}\right), \mathrm{b}\right)$ show how the plateau is formed [44]. From the part (a) of the figure, one can conclude that the spectrum formation is also influenced by the electrons, which tunneled within several periods $T_{0}=2 \pi / \omega_{0}$. The kinetic energy of these electrons, as shown by figure (b) is less than $2.4 U_{p}$, so they participate in the formation of the initial part of the plateau up to

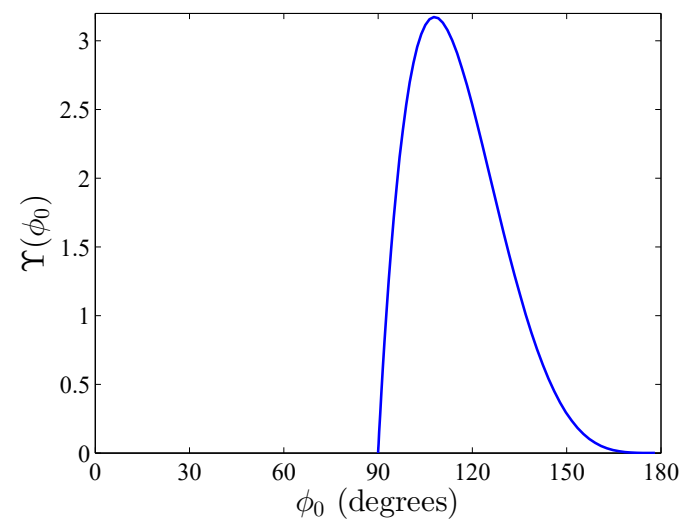

FiguRE 1.10: Factor $\Upsilon$ in the expression for the kinetic energy gained by a free electron in the electromagnetic field as a function of the wave phase at ionization, $\phi_{0}$.

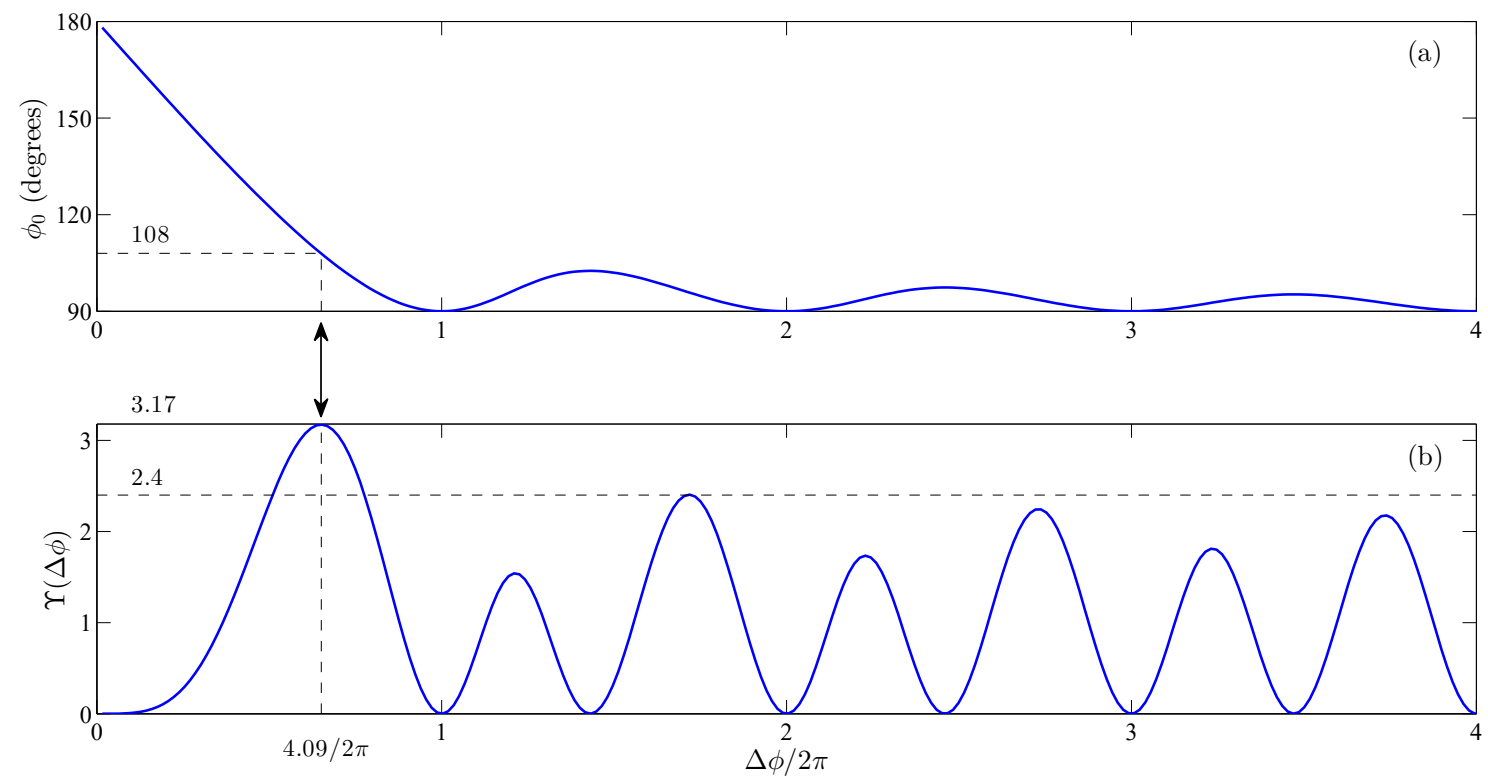

FiguRE 1.11: (a) The relationship between the phase at the time of electron tunneling and the time of the electron free motion, divided by the period of laser radiation: $\Delta \phi / 2 \pi=$ $\left(t_{R}-t_{0}\right) / T_{0}$. (b) The dependence of the acquired kinetic energy on the time of the electron free motion, divided by $T_{0}$. 
$2.4 / 3.17 \approx 75 \%$. Note that the aforementioned HHG theory refers to one half-cycle of the laser field, but an electron can tunnel not only immediately after the positive maximum of the field (as shown in the Fig. 1.9), but also after a negative, twice per period. Thus, due to periodicity of the laser field, the high harmonic radiation consists of brief pulses separated each other by time $T_{0} / 2$, which correspond to the moments of the electron returns to the parent nucleus. According to such a qualitative description, we can represent the intensity of the atom response, a squared polarization vector $P(t)$, as an initial laser pulse $e^{i \omega_{0} t}$ modulated by a periodical function. For instance, we have

$$
|P(t)|^{2} \propto\left|e^{i \omega_{0} t} \sum_{n=1}^{N_{\max }} \delta\left(t-n T_{0} / 2\right)\right|^{2}
$$

where $N_{\max }$ denotes the maximal number of harmonics (1.95) and the set of delta functions (known as Dirac comb) approximates the instant intense pulses. Hence the Fourier transform of the signal contains harmonics spaced by $2 \omega_{0}$ (see Fig.1.12):

$$
|\widehat{P}(\omega)|^{2}=|\mathcal{F}\{P(t)\}|^{2} \propto \frac{\sin ^{2}\left(\frac{\pi\left(\omega-\omega_{0}\right)\left(N_{\max }-1\right)}{2 \omega_{0}}\right)}{\sin ^{2}\left(\frac{\pi\left(\omega-\omega_{0}\right)}{2 \omega_{0}}\right)} .
$$

Note that the above expression, as well as (1.96), represents the Dirac comb, since from $(1.97)$ we can see, that $|\widehat{P}(\omega)|^{2} \propto\left(N_{\max }-1\right)^{2} \sum_{n=1}^{N_{\max }} \delta\left(\omega-\omega_{0}(2 n+1)\right)$. In other words, like in perturbative regime, in the nonperturbative one for atom, we expect a harmonic spectrum containing only odd harmonics.

As we see, in spite of all the simplifications, the semi-classic theory gives remarkably accurate inferences. The key assumption of the theory is ignoring of the Coulomb potential with respect to the wave field. Note that according to (1.89) the amplitude of the electron oscillations is $x_{0}=E / \omega_{0}^{2}$, so for $I=10^{14} \mathrm{~W} / \mathrm{cm}^{2}$ and $\lambda_{0}=800 \mathrm{~nm}, x_{0} \approx 16$ a.u., i.e. an order of magnitude higher than the atom typical size. Such property justifies ignoring the Coulomb potential in the consideration of 

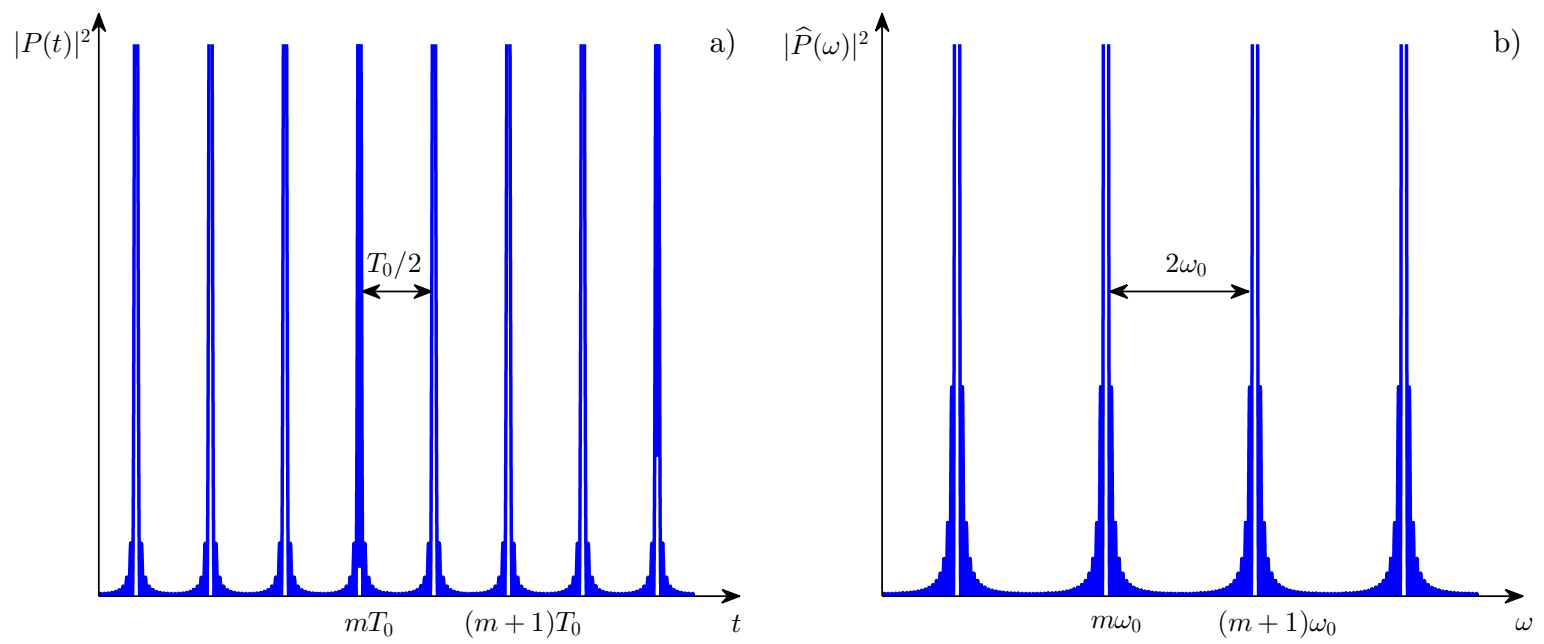

FIGURE 1.12: HHG: atom response in time (a) and spectrum (b).

the free electron motion.

The strong-field approximation of HHG by Lewenstein et al. [73] that followed the semi-classical Corkum's theory, has allowed to include consistently such quantum effects as tunneling, quantum diffusion, quantum interference and phase matching. Similarly to semi-classical theory, the first quantum theory of HHG was based on the electric dipole approximation, which implies that the main term in the multipole expansion of the interaction potential is due to the dipole moment of the system [68]. The dipole approximation is correct if a characteristic size of the system, e.g. the atomic/molecule size of the order $a_{0}$, is much less than the scale of nonhomogeneity of the field, e.g. the wavelength $\lambda \gg a_{0}$. The specification "electric" means that we take into account only the electrical part of the Lorentz force, whereas the magnetic part is neglected. The assumption is not always justified, see e.g. [8] about the molecular attomagnetism, although it is still appropriate for the effects we are studying here.

The quantum expectation value for the dipole moment reads (sign comes from the 
negative electron charge):

$$
\mathbf{d}(t)=-\int_{\mathbb{R}^{3}} \psi^{*}(\mathbf{x}, t) \hat{\mathbf{x}} \psi(\mathbf{x}, t) d \mathbf{x},
$$

where $\psi(\mathbf{x}, t)$ is the wavefunction of an electron, operator $\hat{\mathbf{x}} \equiv \mathbf{x}$, and $d \mathbf{x}$ stands for the volume integration. In the Strong Field Approximation (SFA), the wavefunction is decomposed into the function of the ground state $|0\rangle$ (as in [73] we use here Dirac bra-ket notation) with amplitude $a(t)$ (the contribution of other bound states is neglected) and the integral taken by continuum states of the outgoing electron $|\mathbf{v}\rangle$, with transition amplitude denoted as $b(\mathbf{v}, t)$ :

$$
|\psi(\mathbf{x}, t)\rangle=e^{\mathrm{i} I_{p} T}\left(a(t)|0\rangle+\int b(\mathbf{v}, t)|\mathbf{v}\rangle d \mathbf{v}\right)
$$

where $d \mathbf{v}$ stand for the integration over all the velocity space, i.e. continuum. In case of LP electric field $E_{x}(t)=E_{0} \cos (\omega t)$ and on the assumption of the low ionization level $(a(t) \simeq 1)$, the Schrödinger equation can be exactly solved for $b(\mathbf{v}, t)$ (through the integral). Then the expression for a time-dependent dipole moment is [73]:

$$
\mathbf{d}(t)=\mathrm{i} \int_{0}^{t} \int E_{0} \cos \left(\omega t^{\prime}\right) d_{x}\left(\mathbf{p}-\mathbf{A}\left(t^{\prime}\right) / c\right) d_{x}^{*}(\mathbf{p}-\mathbf{A}(t) / c) e^{\mathrm{i} S\left(\mathbf{p}, t, t^{\prime}\right)} d \mathbf{p} d t^{\prime}+\text { c.c },
$$

which depends on the quasi-classical action:

$$
S\left(\mathbf{p}, t, t^{\prime}\right)=\int_{t^{\prime}}^{t}\left(\frac{\left[\mathbf{p}-\mathbf{A}\left(t^{\prime \prime}\right) / c\right]^{2}}{2}+I_{p}\right) d t^{\prime \prime},
$$

via a factor $e^{\mathbf{i} S\left(\mathbf{p}, t, t^{\prime}\right)}$ accounting for the phase incursion during the free motion of the electron between times $t^{\prime}$ and $t$. In formulae (1.100), (1.101): canonical momentum $\mathbf{p}=\mathbf{v}+\mathbf{A}(t) / c$, where a vector potential $\mathbf{A}(t)=\left(-E_{0} \sin (\omega t), 0,0\right)$, and $d_{x}$ is the element of the atomic dipole matrix for bound-free transition, "c.c." is the abbrevi- 
ation for "complex conjugate". Note first, that the inner integral of (1.100) with the exponent function (1.101) can be identified as a generalized Fourier integral

$$
I(x)=\int_{a}^{b} f(t) e^{i x \varphi(t)} d t
$$

If so, one can apply the method of stationary phase [19] to obtain the leading asymptotic behaviour that comes from the stationary points of the real function $\varphi$, if any. According to the Riemann-Lebesgue lemma the other parts of the integral go to zero, provided that the integral $\int_{a}^{b}|f(t)| d t$ is convergent and $\varphi(t)$ is continuously differentiable. In our case the condition of the phase stationarity should be set for the action as

$$
\nabla_{\mathbf{p}} S\left(\mathbf{p}, t, t^{\prime}\right)=0
$$

Then from the expression (1.101), $\nabla_{\mathbf{p}} S\left(\mathbf{p}, t, t^{\prime}\right)=\int_{t^{\prime}}^{t}\left[\mathbf{p}-\mathbf{A}\left(t^{\prime \prime}\right) / c\right] d t^{\prime \prime}=\int_{t^{\prime}}^{t} \mathbf{v} d t^{\prime \prime}=$ $\mathbf{x}(t)-\mathbf{x}\left(t^{\prime}\right)=0$, in other words $\mathbf{x}(t)=\mathbf{x}\left(t^{\prime}\right)$. Thus, the major contribution into the integral (1.100) corresponds to quantum "trajectories" that coincide with the classical ones: they start near the atom at time $t^{\prime}$, and return there at time $t$ [73].

Along with confirmation and further development of the semi-classical approach outcomes, the quantum analysis allows to obtain the phase-matching conditions for HHG. As we know from the above analysis of the third-harmonic generation (see (1.67), (1.68) and Fig. 1.4), this condition is extremely important for the efficiency of the process. As also mentioned, see (1.68) and further, the cause of violation of phase-matching is the dispersion of the waves in the medium, not only in a neutral gas, but also in a plasma. Meanwhile, it is difficult to balance the dispersion influence in an isotropic medium, especially as there is also a specific phase incursion between the dipole moment and the laser field, see (1.100). However, the analysis and computations performed by Balcou at al. [6] found out that some specific geometries of the laser beam can help to achieve the fulfilment of the phase-matching conditions, 
at least locally.

To conclude this section, we return to the problem of the HHG induced by CP laser fields. It was established in 1980s that in the perturbative regime CP laser field stimulate generation of the circularly-polarized harmonics [86]. However, this inference is not true in the nonperturbative regime. Meanwhile, there was (and still exists) a practical interest in the generation of attosecond circularly polarized harmonics, see e.g. [8]. It follows from the semi-classical theory of HHG that in case of $\mathrm{CP}$, the tunneled electron never returns to the parent nucleus of an atom. However, several ideas and methods have been proposed to circumvent this statement. It was predicted that the efficiency of the HHG process can be improved by using molecules and molecular ions that are more easily polarized than atoms, and hence can serve as better intense source of harmonics [53]. The corresponding process is referred as Molecular High-Order Harmonic Generation (MHOHG) [7]. Also, soon after the nonperturbative quantum theory of HHG by LP pulse came into existence, it was generalized on the case of elliptically polarized laser pulse [2]. The theory suggests controlling the phase of atomic polarizability using the geometry of the focused laser beam (when its waist is much greater then size of the nonlinearity), just as it was done for the case of linearly polarized field [6]. In number of studies, in order to control the HHG of the CP subfemtosecond and attosecond pulses, it was proposed to use a special combination of electric fields to enhance recollisions with neighboring ions. In particular, it may be done as a superposition of counter-rotating laser pulses of different frequencies (bichromatic) [54,89, 125]. Another way involves using intense laser pulses together with static field, which contributes to recollision of the electron with parent ions [126]. Even semi-classical calculations conducted within of these models showed that the width of the plateau depends on the field configuration. For instance, the numerical factor preceeding $U_{p}$ in (1.95), normally is larger than 3.17. The shift of the cut-off to the higher harmonics makes short and intense CP 
laser pulses an attractive tool of investigation of the electron dynamics in atoms or molecules. Note that the mentioned semi-classical models are only the starting point of the study, all the above assumptions were verified by numerical simulations of the Schrödinger equation.

\subsubsection{Laser Filamentation: Standard Models.}

Filamentation is a phenomenon of self-channeling of intense laser pulses in gases, which was discovered by Braun and Mourou [28] in 1995. As seen in Section 1.3.2, the laser beam undergoes self-focusing while propagating in the gas. In the simplified model [48], the beam can collapse to zero thickness while its intensity goes to infinity. In reality, before this happens, the gas is partially ionized due to multiphoton ionization. Free electrons form a plasma, which has a lower refraction index than the neutral gas. Indeed, in case of the relatively high frequencies, for transverse waves in weakly ionized collisionless plasmas, neglecting the electron temperature and taking into account that the ratio of the electron mass to the proton mass is very small, $m_{e} / m_{p} \ll 1$, makes the permittivity take the form [70]:

$$
\epsilon(\omega)=1-\frac{\omega_{p e}^{2}}{\omega^{2}}
$$

where in a.u. $\omega_{p e}^{2}=4 \pi \mathcal{N}_{e}$ is the electron plasma frequency, $\mathcal{N}_{e}=\mu \mathcal{N}_{0}$ is the electron number density, expressed through the gas number density $\mathcal{N}_{0}$ and a factor $1 \geq \mu \geq 0$ characterizing the degree of ionization. Thus, the formation of free electrons plasma makes a negative contribution to the refractive index [24]. In fact, without plasma it is obvious that $n(\omega) \equiv n_{0}=1$, then from $n^{2}(\omega)=\left(n_{0}+\Delta n(\omega)\right)^{2}=\epsilon(\omega)$ it follows $\Delta n(\omega) \approx-\omega_{p e}^{2} / 2 \omega^{2}$. For example in the case of a laser pulse wavelength $\lambda_{0}=800 \mathrm{~nm}$, i.e. $\omega_{0}=0.057$ a.u., and for a gas number density $\mathcal{N}_{0}=3.5 \times 10^{20}$ $\mathrm{mol} / \mathrm{cm}^{3}=5.17 \times 10^{-5}$ a.u., one gets $\epsilon\left(\omega_{0}\right)=1-0.2 \mu$ or $\Delta n\left(\omega_{0}\right) \approx-0.1 \mu$. 
Therefore, the plasma behaves like a defocusing lens (the optical depth is bigger for the marginal ray than for the central one in contrast to the self-focusing case (1.78)), resulting in the decrease of the laser beam intensity, which becomes too low to ionize the surrounding neutrals. In the neutral Kerr medium, the self-focusing resumes, and then the inspissated laser beam causes the plasma formation, thus, the cycle repeats "periodically", see Fig. 1.13. The faithful balance between the focusing and diffraction leads to the formation of a so-called filament.

An important remark, which must be done here, in addition to its high-intensity, the laser pulse should be short in duration of the order of tens of femtoseconds $\left(10^{-15}\right.$ s). Otherwise, instead of multiphoton ionization of the on-site medium, the cascade ionization (also referred as electron avalanche) can occur [24,33,106]. The density of the free electrons becomes so high that they induce ionization by colliding with neutrals located even far away from the laser beam path. This upsets the balance between self-focusing and defocusing: in such a plasma the laser beam will diverge only.

Now we proceed to a brief review of mathematical models used to study the

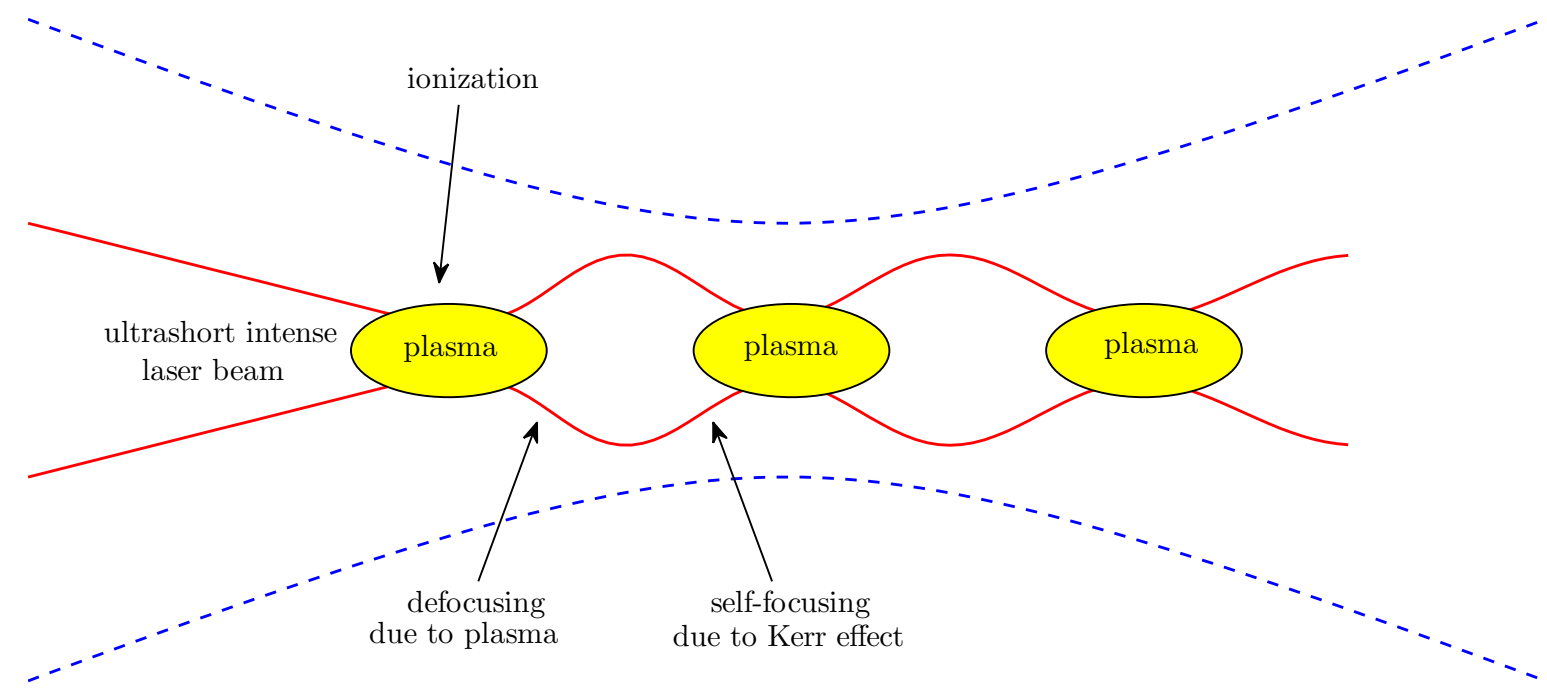

FIGURE 1.13: Laser filamentation: qualitative model. 
essentially time-dependent, so the NLSE in the form (1.85), which we used before to describe the "stationary" self-trapping of light (or quasi-stationary if one introduces instead of $z$ a new variable $z-c t$ ), is not in principle valid here. The fully selfconsistent mathematical model must include side by side [79, 82]

- the set of time-dependent Schrödinger equations (TDSEs), which describes the interaction of the atoms/ions with an electro-magnetic field of the laser pulse;

- Maxwell's equations (MEs) modeling the evolution of the external field under the response of the molecules of gas in the region where the filament propagates;

- the kinetic equations, in order to take into account the dynamics of the freeelectron plasma.

In practice, the realization of the above model faces the extremely high computational cost for solving the system of TDSEs, and requires high performance computing. Nevertheless, over the past 10 years important results have been achieved within this model, and the studies are in progress, see e.g. [79,81,84]. In particular, this thesis is devoted to further research within this non-standard (for the nonlinear optics community) model, whose detailed description will be provided in Chapter 2 . We are discussing here the principal features of the standard models used to study filamentation $[12,21,62]$.

In a broad sense, there are two general standard approaches to describe the shortpulse propagation in a nonlinear medium [93,98]:

- models considering the evolution of the envelope of the EM-wave. As a rule, they are based on time-dependent NLSE, and the second generation equations, e.g. the nonlinear envelope equation (NEE) [26];

- models based on full electric field propagators. It is the set of equations connected with Korteveg-de Vries (KdV) equation, e.g. the short pulse evolu- 
tion equation (SPEE) [93] or the unidirectional pulse propagation equation (UPPE) [98].

As applied to nonlinear optics, both "parental" equations (NLS and KdV), as well as their descendants, are reductions obtained under some assumptions from the MEs, and are similar to those used to derive (1.85). To describe the linear and nonlinear response of the medium, we use the expansion (1.8) with the susceptibility coefficients $\chi^{(2 m+1)}$. In the strict sense, the latter allows to classify standard models as perturbative, even if they use several (i.e. not only $\mathbf{P}^{(3)}$ ) nonlinear terms in the expansion (1.65), resulting in the so-called higher order Kerr effect (HOKE) models [62].

Finally, to describe the density of free electrons in time $\rho(t)$, the standard models are usually empirically coupled with the following phenomenological equation $[21,62]$ :

$$
\partial_{t} \rho=W(I)\left(\rho_{\mathrm{at}}-\rho\right)+\frac{\sigma}{I_{\mathrm{p}}} \rho I-f(\rho)
$$

where (i) in the first term of the RHS $\rho_{\text {at }}$ is the a neutral gas number density, and the function $W(I)$ gives the intensity $(I)$-depending rate of the multi-photon (tunnel) ionization (see the Section 1.4.1) based on Keldysh's theory [58] and tabulated in the review paper by Couairon and Mysyrowicz [38]; (ii) the second term of the RHS describes the avalanche ionization [106], whose rate is proportional to the cross-section $\sigma$ of the inverse bremsstrahlung (the process of absorbing a laser photon by the electron, which is feasible if the part of the momentum and energy can be transmitted to an atom/ion due to scattering [106]) and is inversely proportional to the ionization potential $I_{\mathrm{p}}$; (iii) finally, the function $f(\rho)$ describes the electron recombination with parent or neighboring ions.

Now let us go back to the above nonlinear optics equations used to describe the laser pulse evolution. Let us start with the envelope description.

\section{Envelope modeling.}


Although the NLSE is a very useful tool in many nonlinear optics applications, it is sometimes a priori not a satisfactory model for short pulses, in particular, to deal with phenomena such as laser filamentation $[21,62,93,94]$. It is well-known that NLSE is universal when a monochromatic weakly nonlinear envelope propagates in a strongly dispersive transparent medium [94]. Following the notation from [93], we choose the carrier wave as $\Phi(\mathbf{x}, t)=\operatorname{Re}\left\{A(\mathbf{x}, t) e^{\mathrm{i}\left(\mathbf{k}_{\mathbf{0}} \cdot \mathbf{x}-\omega\left(\mathbf{k}_{\mathbf{0}}\right) t\right)}\right\}$ and let a small parameter $\varepsilon$ characterize both the weak nonlinearity of the complex envelope $A(\mathbf{x}, t)$ and its bandwidth. Then, the evolution equation for the envelope writes in the form

$$
\partial_{t} A+\nabla_{\mathbf{k}} \omega \cdot \nabla A-\frac{\mathbf{i} \varepsilon}{2} \nabla^{\top} \Omega \nabla A-\mathbf{i} \varepsilon n_{2} \frac{c}{2 \pi}\left(\mathbf{k} \cdot \nabla_{\mathbf{k}} \omega\right) A|A|^{2}=0
$$

where $\nabla_{\mathbf{k}} \omega$ is the group velocity of the carrier wavevector $\mathbf{k}_{0}, \Omega=\partial_{k_{i}, k_{j}}^{2} \omega$ is the group velocity dispersion tensor $\left(k_{i}, k_{j}=\left\{k_{x}, k_{y}, k_{z}\right\}\right)$, and the system is called strongly dispersive when the eigenvalues of $\Omega$ are nonzero, $n_{2}$ is the nonlinear correction to the refraction index $(1.62), \nabla\left(\nabla^{\top}\right)$ is the column (row) vector $\left(\partial_{x}, \partial_{y}, \partial_{z}\right)$. Thus, physically, the first two terms of the equation (1.105) describe a transport of the envelope, the third one accounts for its dispersion (spreading in time and space), while the last nonlinear term matches up to the optical Kerr effect (as it is evident from comparison with (1.58) and further). The analysis of the equation in 1-d case confirms that the interplay between dispersion and nonlinearity can prevent a wavepacket from spreading and results in a stable soliton solution for the envelope [94] (see also the equation (1.58) and its solution (1.59) with formal substitution $z=t$ ). However, in higher dimensions, $N \geq 2$, the focusing properties of the equation (1.105) are amplified and the solutions can blow up in finite time [92]. Moreover, there is another instability occurring, due to the odd harmonic generation in gases. In the case of weak dispersion, the field can be expanded around the carrier frequency $\omega_{0}$, as $\Phi(\mathbf{x}, t)=\operatorname{Re}\left\{A(\mathbf{x}, t) e^{\mathbf{i}\left(\mathbf{k}_{0} \cdot \mathbf{x}-\omega_{0} t\right)}\right\}+$ corrections. Each of the last is referred to 
the $(2 m-1)^{\text {th }}$ harmonic, and is equal to $A^{2 m-1} /\left[\omega\left((2 m-1) k_{0}\right)-(2 m-1) \omega\left(k_{0}\right)\right]$, $m=1,2 \ldots$ [93]. Thus, in the case of effective harmonic generation, the denominators go to 0 and hence the corrections become as large as the leading order approximation.

However, the special generalizations of NLSE, such as the nonlinear envelope equation (NEE), allowed to correct the mentioned shortcomings of the original equation for ultrafast pulses [26]. The NEE approach includes (i) the effects of high-order dispersion having used the differential operator:

$$
\widetilde{D}:=\sum_{n=2}^{\infty} \frac{k^{(n)}(\omega)}{n !}\left(\mathrm{i} \partial_{t}\right)^{n},
$$

and (ii) space-time coupling operator:

$$
T:=\left(1+\frac{i}{\omega_{0}} \partial_{\tau}\right),
$$

where new variables $z^{\prime}=z$ and $\tau=t-z / v_{g}$ set a retarded time frame, and $v_{g}=$ $\partial_{k} \omega$ is the group velocity. For example, in the lowest approximation in nonlinearity (considering only $\chi^{(3)}$-term), the generalized equation takes the form [24]:

$$
\begin{aligned}
\triangle_{\perp} A & +2 \mathrm{i} k_{0} \partial_{z^{\prime}}\left[\left(1+\frac{\mathrm{i}}{\omega_{0}} \partial_{\tau}\right)+2 k_{0} \widetilde{D}\left(1+\frac{\mathrm{i}}{\omega_{0}} \partial_{\tau}\right)\right] A+ \\
& +\frac{3 \omega_{0}^{2} \chi^{(3)}}{c^{2}}\left[1+\left(2+\frac{\omega_{0}}{\chi^{(3)}\left(\omega_{0}\right)} \frac{d \chi^{(3)}}{d \omega}\right) \frac{\dot{i}}{\omega_{0}} \partial_{\tau}\right]\left|A^{2}\right| A=0,
\end{aligned}
$$

where the first two terms (i.e. the first row) capture to time-space focusing effect due to presence of the operator $T$, while the self-steepening (see Appendix B) of the incident optical pulse is described through the time-coupling factor incorporated in the last nonlinear term $[21,24,62]$. As a result, in contrast to NLSE, the NEE can handle optical pulses with broad spectra and fast temporal features.

In other words, the fact that in the corresponding physical experiments the blowup of solutions is not necessarily observed, stimulates the search for new ingredients 
for the envelope equations, which would allow a more accurate description of filamentation. Another model modifying the NLSE has recently been presented by Dumas et al. [43]. The model incorporates many physical phenomena neglected by NLSE (1.105), such as the self-steepening of the pulse (also included in NEE), damping, off-axis variations of the group velocity, photo- and collisional ionization processes. The authors rigorously justified that an exact solution of their NLSE-modified problem exists and is unique (with initial condition), on the time interval $[0, T / \varepsilon]$, where $T>0$ and $1 \gg \varepsilon>0$ is a small scaling parameter in the model. Thus, the modified equations are justified far from the focusing time $T_{\text {foc }}$, in contrast with NLSE (1.105), which is bounded only on the time interval $[0, T]$, with $T<T_{\text {foc }}$.

It is worth noting that despite the fact that it is possible to analyse the advanced (with respect to NLSE) nonlinear equations derived from the MEs and even add into them new ingredients, these equations allow numerical solutions only. Even so, the numerical computations for the envelope models are less costly in time and resources compared to the Maxwell-Schrödinger models, see Chapter 2.

\section{Full electric field propagators.}

Under this heading we will refer to two important examples: the short pulse evolution equation (SPEE) [65], and the unidirectional pulse propagation equation (UPPE). Intrinsically SPEE is the modified Kadomtsev-Petviashvili (MKP) equation [93]:

$$
\partial_{\zeta}\left(\partial_{\tau} v+6 v^{2} \partial_{\zeta} v+\partial_{\zeta \zeta \zeta}^{3} v\right)=\triangle_{\perp} v
$$

which describes the evolution of Riemann invariants (functions of the variables of a hyperbolic equation, which propagate without changes along the characteristic curves of the PDE) [121], in case of propagation in the positive direction of $x: v(\zeta, \tau)=$ $F(\zeta=x-c t, \tau=\varepsilon t)$, and also under (i) the additional influence of weak, order of 
$\varepsilon(1 \gg \varepsilon>0)$ cubic nonlinearity (the second term in the LHS), (ii) weak dispersion (LHS third term) and (iii) weak diffusion in both transverse directions (RHS of the equation). Applying the Fourier transform to the wave equation $\nabla \times[\nabla \times \mathbf{E}]=$ $-c^{-2} \partial_{t t}^{2} \mathbf{D}$, see (1.32), in the case of LP field, such that $\mathbf{E}\left(\mathbf{x}_{\perp}, z, t\right)=\mathbf{e}_{y} E\left(\mathbf{x}_{\perp}, z, t\right)$, followed by subsequent multiple scale analysis eliminating the secular terms [19], it is possible to obtain the FT of SPEE for the leading order field amplitude [93]. The inverse FT, together with introducing the convenient length scale ratios finally results in the SPEE:

$$
\partial_{\tau}\left(\partial_{z} E+\varepsilon_{\mathrm{NL}} E^{2} \partial_{\tau} E-s \varepsilon_{\text {disp }} \partial_{\tau \tau \tau}^{3} E-\varepsilon_{\text {att }} \partial_{\tau \tau}^{2} E\right)=\varepsilon_{\text {diff }} \nabla_{\perp}^{2} E
$$

where the set of small parameters $1 \gg \varepsilon_{\mathrm{NL}}, \varepsilon_{\mathrm{disp}}, \varepsilon_{\mathrm{att}}, \varepsilon_{\mathrm{diff}}$ describes the influence of the weak nonlinearity, dispersion, attenuation and diffusion of the field; also the parameter $s= \pm 1$ defines the sign of the dispersive term: normal $\left(\partial_{\omega} n(\omega)>0\right)$ or anomalous $\left(\partial_{\omega} n(\omega)<0\right)$. The analysis of $(1.110)$ showed that the regularization due to dispersion and attenuation can lead to the optical shocks formation, see Appendix $\mathrm{B},[65,93]$.

Recall again that the SPEE is an equation for the field amplitude, so in such approach the shape of the pulse can be rather short (weak dispersion is assumed) and hence have a broad spectrum. Thus, this equation can describe the collective behaviour of all odd harmonics without the blow-up observed in case of NLSE. To conclude with the SPEE, we notice that due to its clarity, the equation can help to understand more complicated models such as UPPE [93].

The last model (UPPE) belongs to fully spectral solvers [62,98]. It is a coupled 
pair of the forward-propagating wave

$$
\begin{aligned}
& \partial_{z} \mathbf{E}_{k_{x}, k_{y},+}^{\perp}(\omega, z)=i k_{z} \mathbf{E}_{k_{x}, k_{y},+}^{\perp}(\omega, z)+ \\
& \quad+\sum_{s=1,2} \mathbf{e}_{s}^{\perp}\left(\mathbf{e}_{s} \cdot\left[\frac{2 \pi i \omega^{2}}{c^{2} k_{z}} \mathbf{P}_{k_{x}, k_{y}}^{\perp}(\omega, z)-\frac{2 \pi \omega}{c^{2} k_{z}} \mathbf{J}_{k_{x}, k_{y}}^{\perp}(\omega, z)\right]\right)
\end{aligned}
$$

and the backward-propagating wave

$$
\begin{aligned}
& \partial_{z} \mathbf{E}_{k_{x}, k_{y},-}^{\perp}(\omega, z)=-i k_{z} \mathbf{E}_{k_{x}, k_{y},+}^{\perp}(\omega, z)- \\
& \quad-\sum_{s=1,2} \mathbf{e}_{s}^{\perp}\left(\mathbf{e}_{s} \cdot\left[\frac{2 \pi i \omega^{2}}{c^{2} k_{z}} \mathbf{P}_{k_{x}, k_{y}}^{\perp}(\omega, z)-\frac{2 \pi \omega}{c^{2} k_{z}} \mathbf{J}_{k_{x}, k_{y}}^{\perp}(\omega, z)\right]\right),
\end{aligned}
$$

derived from Maxwell's equations [61]. The equations contain summation over the polarization index $s(s=1,2)$, so that $\mathbf{e}_{s}$ are unit polarization vectors normal to the wave vector $\mathbf{k}=\left(k_{x}, k_{y}, k_{z}\right)$. To compute the longitudinal component of the field, its derivative $\partial_{z} E_{z}$ can be obtained from $\nabla \cdot \mathbf{D}=0$ and using (1.111), (1.112). To close the system, the polarization $\mathbf{P}\left(\mathbf{x}_{\perp}, z, t\right)$ and the current density $\mathbf{J}\left(\mathbf{x}_{\perp}, z, t\right)$ as functions of the electric field $\mathbf{E}\left(\mathbf{x}_{\perp}, z, t\right)$ must be added to (1.111) and (1.112). In the context of terminology, "unidirectional" refers to the medium response, which can be calculated from only the forward portion of the field:

$$
\mathbf{P}\left(\mathbf{E}_{-}+\mathbf{E}_{+}\right), \mathbf{J}\left(\mathbf{E}_{-}+\mathbf{E}_{+}\right) \rightarrow \mathbf{P}\left(\mathbf{E}_{+}\right), \mathbf{J}\left(\mathbf{E}_{+}\right)
$$

Thus, one can take the polarization again from the expansion (1.8), at least up to the second term describing the Kerr effect. As to the current density, it can be grouped together with the electric field by applying the classical Drude model, which (as usual in a.u.) reads

$$
\partial_{t} \mathbf{J}+\nu_{e} \mathbf{J}=\rho \mathbf{E}
$$

where $\nu_{e}$ denotes the electron collision frequency, and $\rho$ is the freed electron density, whose time-evolution is given for example by (1.104). 
It is noteworthy that taking the dispersion relation in the form

$$
k(\omega)=a \omega^{3}-\frac{b}{\omega}+q \omega
$$

where the parameters $a, b$ and $q$ are chosen to accurately reflect the full dispersion profile near a specific frequency, the authors [98] turned from the UPPE to the generalized MKP optical filamentation equation having much in common with SPEE (1.110):

$$
\partial_{\tau}\left(\partial_{z} E-a \partial_{\tau \tau \tau}^{3} E+\frac{4 n_{2}}{3 c} \partial_{\tau} E^{3}+\mathcal{N}_{\mathrm{abs}}(\rho, I) E\right)+\left(b+b_{\text {plas }}(\rho)\right) E=\frac{c}{2 n_{0}} \nabla_{\perp}^{2} E
$$

where $n_{0}$ and $n_{2}$ stand for the refraction indexes, the same as in the expression for Kerr effect (1.62), while

$$
\begin{aligned}
& b_{\text {plas }}(\rho)=\frac{2 \pi}{n_{0} c} \rho, \\
& \mathcal{N}_{\text {abs }}(\rho, I)=\frac{W(I) I_{\mathrm{p}}\left(\rho_{\mathrm{at}}-\rho\right)}{2 I} .
\end{aligned}
$$

The numerical treatment of the UPPE is carried out through the corresponding spectral amplitudes

$$
E_{k_{x}, k_{y}}(\omega, z)=A_{k_{x}, k_{y}} e^{\mathrm{i} K\left(k_{x}, k_{y}, \omega\right) z}
$$

with the linear propagator

$$
K\left(k_{x}, k_{y}, \omega\right)=\sqrt{\omega^{2} \epsilon(\omega) / c^{2}-k_{x}^{2}-k_{y}^{2}}
$$

In this way the UPPE becomes simpler and is represented as a large system of ODEs, which can be solved by using the suitable libraries [62].

To summarize the section, we repeat that the both standard approaches, the envelope equations (like NEE) and the field propagators (like UPPE), are currently widely used to describe the evolution of ultrafast laser pulses and laser filaments propagation. 
Both approaches have their pros and cons, and are still subject for debate. The corresponding numerical models are relatively fast, although NEE-type models are even faster than the field propagators [98]. On the other hand, the envelope approach has intrinsic limitations related to its dispersion operator. Although the UPPE is free of such flaw, it is subject to another kind of inaccuracy. Namely, the UPPE receives the necessary data about the chromatic properties of the medium, e.g. its permittivity $\epsilon(\omega)$, as an external function. For the purpose of computation reliability, the function must be accurate and free of numerical interpolation artifacts, which cannot always be guaranteed [98].

Finally we emphasize again that, despite of the quite reliable description of the evolution of the ultrafast laser pulses and relatively low computational cost, both standard approaches (i) use approximate but not exact propagation equations; (ii) employ perturbative expansion for the polarization; (iii) receive the frequency-property of medium as an external parameters; (iv) compute free electron density phenomenologically. All these properties suggest that the solution of the problem can be improved and the involvement of the non-standard nonperturbative models will be highly useful. 


\section{Chapter 2}

\section{Maxwell-Schrödinger-Plasma}

\section{Models}

\subsection{Introductory Remarks}

The strong interest in modelling of parameters of the attosecond pulses and laser filaments that arose in 1990s - 2000s has led to the need for an appropriate description of the evolution of ultrashort, only few-optical-cycle, pulses. Clearly that perturbative methods based on the approximate equations, e.g. NLSE-type, although advantageous for not too short pulses, have essential intrinsic restrictions as applied to ultrashort pulses. These issues, which were discussed in Section 1.4.2, were addressed in some key papers $[27,50,55]$. Over the years, several models were proposed, and we refer to a set of review papers $[21,38,62]$. For example, some complex models (HOKE, UPPE [5,61]) allow for accurate simulations and analysis of laser filamentation in some physical regimes $[5,20,30,63,107,119]$. However, an appropriate modeling of the free-electron plasma generated by the ultrashort intense laser pulse, as well as of nonperturbative evolution of this pulse in nonlinear medium, is still an open problem that is only partially addressed in $[17,18,59,90,99,103,109,112]$. 


\subsection{Maxwell-Schrödinger-Plasma Model}

We start with a micro-macro model constituted by Maxwell's equations coupled with several Schrödinger equations, modeling the nonlinear response of gas subject to an electromagnetic (EM) field $[79,81,82]$. We will consider the model in the dipole approximation, which is valid when the smallest internal wavelengths $\lambda_{\min }$ of the electromagnetic field are much larger than the molecule size $\ell$ (in the same direction), that is $\ell \ll \lambda_{\min }$. Typically we have $\lambda_{\min } \approx 800 \mathrm{~nm}$ (Ti:Sapphire laser) and for $\mathrm{H}_{2}^{+}$-molecule size $\ell \approx 2 a_{0} \approx 0.1 \mathrm{~nm}$, see also the Section 1.4.1. To model the macroscopic propagation effects, we define the MEs in the differential form in a bounded spatial domain $\Omega$ with a smooth boundary $\partial \Omega$. We define by $\mathbf{x}^{\prime}=\left(x^{\prime}, y^{\prime}, z^{\prime}\right)^{T}$ the electromagnetic field space variable:

$$
\left\{\begin{array}{l}
\partial_{t} \mathbf{B}\left(\mathbf{x}^{\prime}, t\right)=-c \nabla \times \mathbf{E}\left(\mathbf{x}^{\prime}, t\right) \\
\partial_{t} \mathbf{E}\left(\mathbf{x}^{\prime}, t\right)=c \nabla \times \mathbf{B}\left(\mathbf{x}^{\prime}, t\right)-4 \pi\left(\partial_{t} \mathbf{P}\left(\mathbf{x}^{\prime}, t\right)+\mathbf{J}\left(\mathbf{x}^{\prime}, t\right)\right), \\
\nabla \cdot \mathbf{B}\left(\mathbf{x}^{\prime}, t\right)=0 \\
\nabla \cdot\left(\mathbf{E}\left(\mathbf{x}^{\prime}, t\right)+4 \pi \mathbf{P}\left(\mathbf{x}^{\prime}, t\right)\right)=4 \pi\left(\sum_{q=1}^{2} q \mathcal{N}_{q}\left(\mathbf{x}^{\prime}, t\right)-\mathcal{N}_{e}\left(\mathbf{x}^{\prime}, t\right)\right),
\end{array}\right.
$$

where $\mathcal{N}_{q}\left(\mathbf{x}^{\prime}, t\right)$ and $\mathcal{N}_{e}\left(\mathbf{x}^{\prime}, t\right)$ respectively represent the densities of ions with the charge $q$ (in the model $q=1,2$ ) and of electrons. The initial ionic molecule density is taken to be smooth in space, and is denoted by $\mathcal{N}\left(\mathbf{x}^{\prime}\right)$. It must be approximately equal to the initial electrons number density $\mathcal{N}_{e 0}\left(\mathbf{x}^{\prime}\right)[32]$, and can be constant in time due to the expected low level of ionization. Now, to compute from (2.1), the EM-field vectors $\mathbf{E}\left(\mathbf{x}^{\prime}, t\right)$ and $\mathbf{B}\left(\mathbf{x}^{\prime}, t\right)$, we need to determine the polarization $\mathbf{P}\left(\mathbf{x}^{\prime}, t\right)$ and the current density $\mathbf{J}\left(\mathbf{x}^{\prime}, t\right)$ vectors.

At the molecular (microscopic) scale, we will denote by $\mathbf{x}=(x, y, z)^{T}$ the TDSE space variable (that is for electrons). Before we write down the equations, let us make two remarks. First, we explain the choice of $\mathrm{H}_{2}^{+}$molecule for illustrating the 
MASP model. The exact analytic solution of the Schrödinger equation exists only for the hydrogen atom. For $n$-electron atoms $(n \geq 3)$, one should use methods of self-consistent field, such as Hartree-Fock method [69], or Density Functional Theory (DFT) [60], which result in the essentially approximate form of Schrödinger equation. Switching from atoms to molecules makes the situation even more complex, while the mathematical description of the problem is possible only in the approximate form. In this context the simplest molecule $\mathrm{H}_{2}^{+}$, possesses two advantages: it has only one electron and is symmetric with respect to the point bisecting the interval between the nucleus. It follows the symmetry of the Hamiltonian with respect to the electron coordinate inversion: $\mathbf{x} \longrightarrow-\mathbf{x}$. It is then possible to reduce formally the problem of describing the dynamics of 3 particles, to a 2-body problem (in general, see the second remark below), and hence the exact Schrödinger equation can be used [81]. However, in practice, even more complex molecules like $\mathrm{O}_{2}, \mathrm{~N}_{2}$ could as well be considered within the MASP, using the single active electron (SAE) approximation [105].

The second remark refers to the use of the Born-Oppenheimer approximation (BOA) that assuming the nuclei as a static sources of the field, while the electrons move in such Coulomb potential. Because of the small ratio between the electron and nucleus masses $\left(m_{e} / m_{\text {nucleus }} \leq 1 / 1836\right)$ the reduced mass $\mu \propto m_{e}=1$ a.u., and one can consider Schrödinger equation only for the electron [69], thus reducing the quantum problem to 1-body problem. It is a typical approximation in quantum chemistry, since the time-scale for dynamics of the interatomic electrons (attoseconds) is much shorter then the time-scale for the nuclei motion (femtoseconds) $[31,94,127]$. Although in its whole generality the MASP model for $\mathrm{H}_{2}^{+}$includes the motion of the 3 particles [81], we will use the BOA throughout the thesis. The components of the polarization vector $\mathbf{P}\left(\mathbf{x}^{\prime}, t\right)$, be to substituted into MEs (2.1), are computed using a 
trace operator [79]:

$$
\left\{\begin{array}{l}
\mathbf{P}\left(\mathbf{x}^{\prime}, t\right)=\mathcal{N}\left(\mathbf{x}^{\prime}\right) \sum_{i=1}^{m} \mathbf{P}_{i}\left(\mathbf{x}^{\prime}, t\right)=-\mathcal{N}\left(\mathbf{x}^{\prime}\right) \sum_{i=1}^{m} \chi_{\Omega_{i}}\left(\mathbf{x}^{\prime}\right) \int_{R^{3}} \psi_{i}^{*}(\mathbf{x}, t) \mathbf{x} \psi_{i}(\mathbf{x}, t) d \mathbf{x} \\
\mathrm{i} \partial_{t} \psi_{i}(\mathbf{x}, t)=-\frac{\Delta_{\mathbf{x}}}{2} \psi_{i}(\mathbf{x}, t)+V_{C}(\mathbf{x}) \psi_{i}(\mathbf{x}, t)+\mathbf{x} \cdot \mathbf{E}_{\mathbf{x}_{i}^{\prime}}(t) \psi_{i}(\mathbf{x}, t), \forall i \in\{1, . ., m\}
\end{array}\right.
$$

where $V_{C}$ denotes the interaction potential. For example, let the ionic $\mathrm{H}_{2}^{+}$-molecule be oriented in the plane $(x, y)$ of the selected Cartesian system, then the nuclear potential is written as:

$$
\begin{aligned}
V_{C}\left(R_{0}, \mathbf{x}\right) & =-\left[\left(x-\frac{R_{0}}{2} \cos \theta\right)^{2}+\left(y-\frac{R_{0}}{2} \sin \theta\right)^{2}+z^{2}\right]^{-1 / 2}- \\
- & {\left[\left(x+\frac{R_{0}}{2} \cos \theta\right)^{2}+\left(y+\frac{R_{0}}{2} \sin \theta\right)^{2}+z^{2}\right]^{-1 / 2} }
\end{aligned}
$$

where $\theta \in\left[0^{\circ}, 90^{\circ}\right]$ defines the angle between the molecular axis of $\mathrm{H}_{2}^{+}$and the $x$-axis, with an internuclear distance $R_{0}$; in our computations it equals to 2 a.u., see e.g. [127].

Solving of the TDSEs provides a complete set of the wavefunctions, which in its turn allows to evaluate the ionization level and obtain a continuum spectrum of free electrons propagating in a laser pulse. For LP-pulses such electrons can recombine with the parent ion with maximum energy $I_{p}+3.17 U_{p}$ (see (1.95) and around), or with neighbours with energies exceeding $3 U_{p}[10,35]$. Thus, the spatial domain $\Omega=$ $\cup_{i=1}^{m} \Omega_{i}$, where $\Omega_{i}$ denotes the macroscopic spatial domain containing a molecule of reference associated to a wavefunction $\psi_{i}$, while in $(2.2) \mathbf{P}_{i}(t)=\chi_{\Omega_{i}} \mathbf{d}_{i}(t)$ denotes the macroscopic polarization in this domain, and the index $i$ is associated to the specific coordinate of MEs, $\mathbf{x}^{\prime}$. Functions $\chi_{\Omega_{i}}$ are defined as $\chi \otimes \mathbf{1}_{\Omega_{i}}$ where $\chi$ is a plateau, and $\mathbf{1}_{\Omega_{i}}$ is the characteristic function of $\Omega_{i}$. Also $\mathbf{d}_{i}(t)$ is a microscopic time-dependent dipole moment of a molecule belonging to $\Omega_{i}$ :

$$
\mathbf{d}_{i}(t)=-\int_{\mathbb{R}^{3}} \psi_{i}^{*}(\mathbf{x}, t) \mathbf{x} \psi_{i}(\mathbf{x}, t) d^{3} \mathbf{x}
$$


$\Omega$ MAXWELL EQUATIONS DOMAIN

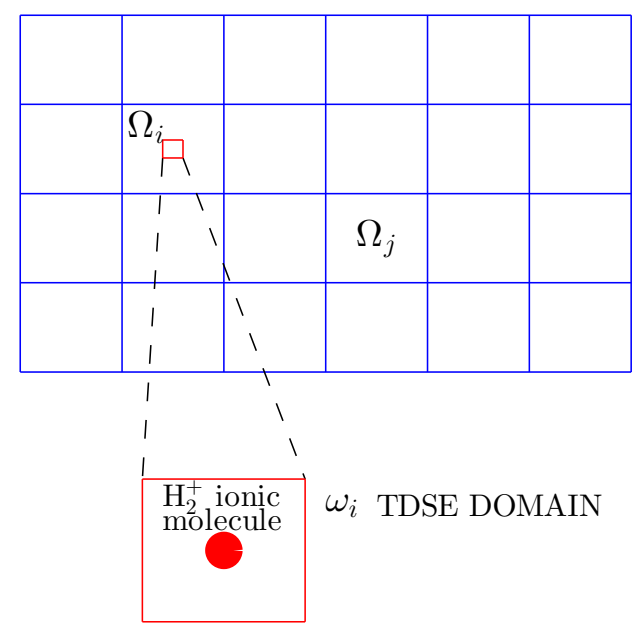

Figure 2.1: Spatial domains for the MASP model. From [77].

In other words, the domain $\Omega_{i}$ contains $\mathcal{N}\left(\mathbf{x}^{\prime}\right) \operatorname{vol}\left(\Omega_{i}\right)$ molecules represented by one single eigenfunction $\psi_{i}$ (under the assumption of a unique pure state [79]). We now assume that the spatial support of $\psi_{i}$ is included in a domain $\omega_{i} \subset \mathbb{R}^{3}$, which is supposed to be sufficiently large, see Fig.2.1. We allow free electrons to reach the boundary $\omega_{i}$ and we impose absorbing boundary conditions on $\partial \omega_{i}$, see Subsection 3.3.3. In the proposed model, the part of the wavefunction absorbed at the boundary generates the plasma of free electrons. Finally $\mathbf{E}_{\mathbf{x}_{i}^{\prime}}$ denotes the electric field (supposed to be constant in space) in $\Omega_{i}$.

To close the set of equations, the current density evolution equation can be integrated in the system via the Drude model (just as in the standard UPPE model, see Section 1.4 .2 and $[21,81])$ on the MEs domain:

$$
\partial_{t} \mathbf{J}\left(\mathbf{x}^{\prime}, t\right)+\nu_{e} \mathbf{J}\left(\mathbf{x}^{\prime}, t\right)=\mathcal{N}_{e}\left(\mathbf{x}^{\prime}, t\right) \mathbf{E}\left(\mathbf{x}^{\prime}, t\right),
$$

where $\nu_{e}$ denotes the effective electron collision frequency. The equation (2.5) is obtained by combining the fluid equations: (i) the equation of continuity, containing 
$\rho$ - electron density, $\mathbf{V}_{e}$ - electron velocity, $\mathcal{S}$ - external plasma sources:

$$
\partial_{t} \rho+\nabla \cdot\left(\rho \mathbf{V}_{e}\right)=\mathcal{S}
$$

and (ii) the equation of Euler written for a "fluid" of free electrons moving under the action of the Lorentz force, and slowing down due to collisions with other electrons:

$$
\partial_{t} \mathbf{V}_{e}+\left(\mathbf{V}_{e} \cdot \nabla\right) \mathbf{V}_{e}=\frac{e}{m_{e}}\left[\mathbf{E}+\frac{\mathbf{V}_{e}}{c} \times \mathbf{B}\right]-\nu_{e} \mathbf{V}_{e}-\frac{\mathcal{S} \mathbf{V}_{e}}{\rho}
$$

To derive the equation for the current density $\mathbf{J}=e \rho \mathbf{V}_{e}$, we multiply (2.6) by $e \mathbf{V}_{e}$ and add with (2.7) multiplied by $e \rho$ :

$$
\partial_{t} \mathbf{J}+\mathbf{V}_{e}(\nabla \cdot \mathbf{J})+(\mathbf{J} \cdot \nabla) \mathbf{V}_{e}=\frac{e^{2} \rho}{m_{e}} \mathbf{E}+\frac{e}{c m_{e}} \mathbf{J} \times \mathbf{B}-\nu_{e} \mathbf{J}
$$

or in the form used in [21]:

$$
\partial_{t} \mathbf{J}+\nu_{e} \mathbf{J}=\frac{e^{2} \rho}{m_{e}} \mathbf{E}+\frac{e}{c m_{e}} \mathbf{J} \times \mathbf{B}-\mathbf{V}_{e}(\nabla \cdot \mathbf{J})-(\mathbf{J} \cdot \nabla) \mathbf{V}_{e}
$$

where the last three terms of the RHS represent the ponderomotive forces, which are important on slowly varying time scale phenomena. In principle, this driving term can induce low plasma currents capable to generate electromagnetic pulses, but this generation becomes very inefficient in gases even for 100 fs laser pulses with intensities of $10^{14} \mathrm{~W} / \mathrm{cm}^{2}$ [21]. Based on these arguments, we neglect the last three terms in (2.8) and come to (2.5) with the electron number density $\mathcal{N}_{e}=\frac{\rho}{m_{e}}$ and $e^{2}=1$ as (2.5) is written in a.u.

In the MASP model $\mathcal{N}_{e}\left(\mathbf{x}^{\prime}, t\right)$ is computed from molecular ionization, specifically

$$
\mathcal{N}_{e}\left(\mathbf{x}^{\prime}, t\right)=\mathcal{N}_{e 0}\left(\mathbf{x}^{\prime}\right)+\mathcal{N}\left(\mathbf{x}^{\prime}\right) \sum_{i=1}^{m} \chi_{\Omega_{i}}\left(\mathbf{x}^{\prime}\right) \mathcal{I}_{i}(t)
$$


where the function

$$
\mathcal{I}_{i}(t)=1-\int_{\Omega_{i}}\left|\psi_{i}(\mathbf{x}, t)\right|^{2} d^{3} \mathbf{x}
$$

defines a fraction of the freed electrons using the $L^{2}$-norm of the bound electron wavefunctions.

Equations (2.1), (2.2) and (2.5) form the MASP model. An important point must be made about the solution method for obtaining the field vectors. The "plasma approximation" is applicable here due to the quasi-neutrality property of the plasma, that is $\mathcal{N}_{1}\left(\mathbf{x}^{\prime}, t\right)+2 \mathcal{N}_{2}\left(\mathbf{x}^{\prime}, t\right) \approx \mathcal{N}_{e}\left(\mathbf{x}^{\prime}, t\right)$, and $\nabla \cdot\left(\mathbf{E}\left(\mathbf{x}^{\prime}, t\right)+4 \pi \mathbf{P}\left(\mathbf{x}^{\prime}, t\right)\right) \approx 0$, i.e. the divergence of the vector of electric displacement is approximately 0 [32]. In other words, the last equation in (2.1) is impractical to obtain $\mathbf{E}\left(\mathbf{x}^{\prime}, t\right)$. Instead, vectors $\mathbf{E}\left(\mathbf{x}^{\prime}, t\right)$ and $\mathbf{B}\left(\mathbf{x}^{\prime}, t\right)$ can be calculated from the first two equations of System (2.1), as we will do using the numerical methods described in Sections 3.1 and 3.2.

The next comment relates to the choice of the Schrödinger equation to describe the medium response. The most complete consideration of a quantum system should be done within the density matrix formalism [24,69], for example, by applying the Maxwell-Bloch equations (MBEs) $[23,71,94]$. The formal procedure for obtaining the polarization $\mathbf{P}$, from the TDSE via the MBEs was described in [81]. It includes (i) finding the first $N$ eigenvalues and eigenfunctions to the Schrödinger equations with the free Hamiltonian (without laser field on this step), (ii) deducing from the obtained eigenfunctions the complex dipolar matrix $\mu \in M_{N}\left(\mathbb{C}^{3}\right)$, (iii) solving the MBEs for the electronic density matrix $\rho=\left(\rho_{j k}\right) \in M_{N}(\mathbb{C})$, and (iv) computing the polarization, as a trace of product of the above matrices $\mathbf{P}=\mathcal{N} \operatorname{tr}(\mu \rho)$. The main issue of this approach is that the numerical computations of the eigenelements of the Hamiltonian operator are very costly. In practice it is limited to no more than 5 energy states, which is far from continuous states (corresponding to ionization) [81]. 


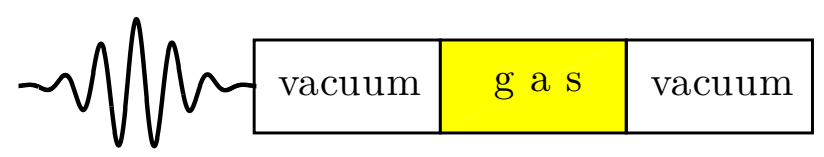

FigURE 2.2: Serial propagation of ultrashort laser pulse through 3 regions.

In order to describe accurately the medium response to a laser pulse in the framework of the MASP model, a very large number of TDSEs is required, increasing immensely the overall complexity of the model. Section 2.3 will address this computational problem. But before turning to this issue, we will specify the choice of the geometry, dimensionality and initial conditions (ICs) of the models studying in Chapter 4.

Schematically the numerical experiment can be described as follows: the EM-pulse propagates in sequence through 3 regions: vacuum, $\mathrm{H}_{2}^{+}$-gas and again vacuum, see Fig. 2.2. As soon as the wave envelope gets into the last vacuum region, the electric field components in space and time are stored, and the spectral intensities and other data can be computed (see Chapter 4).

In general, for 1-electron TDSE and under BOA, the MASP model is 3d-3d in the sense, that it is supposed $\mathbf{E}\left(\mathbf{x}^{\prime}, t\right), \mathbf{B}\left(\mathbf{x}^{\prime}, t\right)$ and $\psi(\mathbf{x}, t)$ are the functions of the all spatial coordinates: $\mathbf{x}^{\prime}=\left(x^{\prime}, y^{\prime}, z^{\prime}\right)^{T}$ and $\mathbf{x}=(x, y, z)^{T}$. In practice, we can reduce the computational complexity of the system by applying a reasonable dimensionality reduction. Note that in notation $M \mathrm{~d}-N \mathrm{~d}, M$ is dimensionality of vector $\mathbf{x}^{\prime}$, and $N$ is dimensionality of $\mathbf{x}$.

\subsubsection{Initial Laser Pulse}

In our numerical computations, we will consider two types of initial data:

- Linearly or circularly polarized laser wavepacket;

- Gaussian beam. 


\section{LP- and CP-wave envelopes and 1d-2d MASP model.}

As we mentioned in Section 1.4.1, short circularly polarized pulses propagating in a molecular gas are subject of a hot topic in the nonlinear optics and photonics communities. For example, in the case of CP-laser pulse the cut-off for HHG process is expected to be in the higher harmonic order, than in the case of LP-pulses. For this reasons in this thesis, we will also consider such pulses as initial condition (ICs) for EM-field.

Consider CP-ultrashort laser pulse propagating in vacuum in the $z^{\prime}$-direction:

$$
\left\{\begin{array}{l}
E_{x^{\prime}}\left(z^{\prime}, t\right)=E_{0 x^{\prime}} f\left(k_{0} z^{\prime}-\omega_{0} t\right) \sin \left(k_{0} z^{\prime}-\omega_{0} t\right), \\
E_{y^{\prime}}\left(z^{\prime}, t\right)=E_{0 y^{\prime}} f\left(k_{0} z^{\prime}-\omega_{0} t\right) \cos \left(k_{0} z^{\prime}-\omega_{0} t\right), \\
B_{x^{\prime}}\left(z^{\prime}, t\right)=-B_{0 x^{\prime}} f\left(k_{0} z^{\prime}-\omega_{0} t\right) \cos \left(k_{0} z^{\prime}-\omega_{0} t\right), \\
B_{y^{\prime}}\left(z^{\prime}, t\right)=B_{0 y^{\prime}} f\left(k_{0} z^{\prime}-\omega_{0} t\right) \sin \left(k_{0} z^{\prime}-\omega_{0} t\right),
\end{array}\right.
$$

where $\omega_{0}$ is the laser frequency, $k_{0}=\omega_{0} / c=2 \pi / \lambda_{0}$ is corresponding wavenumber, and $\lambda_{0}$ its wavelength. The field amplitudes can be written as a function of the pulse intensity $I$ as follows, $E_{0 x^{\prime}}=E_{0 y^{\prime}}=B_{0 x^{\prime}}=B_{0 y^{\prime}}=\sqrt{8 \pi I / c}$ and naturally $E_{0 z^{\prime}}=B_{0 z^{\prime}}=0$. The pulse (2.11) satisfies MEs (2.1) in vacuum, i.e. when $\partial_{t} \mathbf{P}\left(\mathbf{x}^{\prime}, t\right)=0, \mathbf{J}\left(\mathbf{x}^{\prime}, t\right)=0$ and $\mathcal{N}_{q}\left(\mathbf{x}^{\prime}, t\right)=\mathcal{N}_{e}\left(\mathbf{x}^{\prime}, t\right)=0$. Function $f$ is the envelope of the initial electromagnetic field, e.g., in applications considered in Section 4.1, it is chosen as a Gaussian function $f\left(k_{0} z^{\prime}-\omega_{0} t\right)=e^{-\alpha\left(k_{0} z^{\prime}-\omega_{0} t\right)^{2}}$, with some dimensionless constant $\alpha>0$. An example of such pulse is shown in Fig. 2.3.

Note that in order to test the MASP model, we need to compare the numerical results, obtained for LP-fields, with the solution for the perturbative regime theoretical inferences (for example, quadratic dependence of the spectral intensity on the propagation length, see (1.67)). For such cases, we can just set in (2.11), say $E_{0 x^{\prime}}=B_{0 y^{\prime}}=0$.

It is also convenient to use initial LP-laser pulses when working on further devel- 


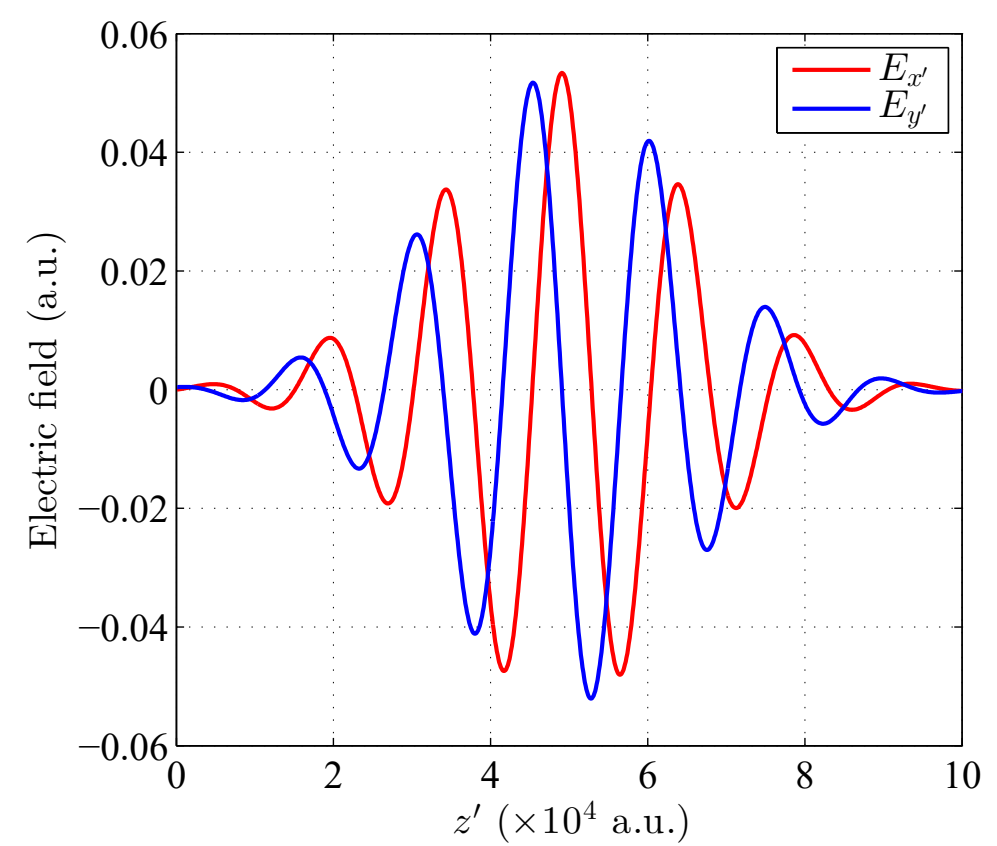

a)

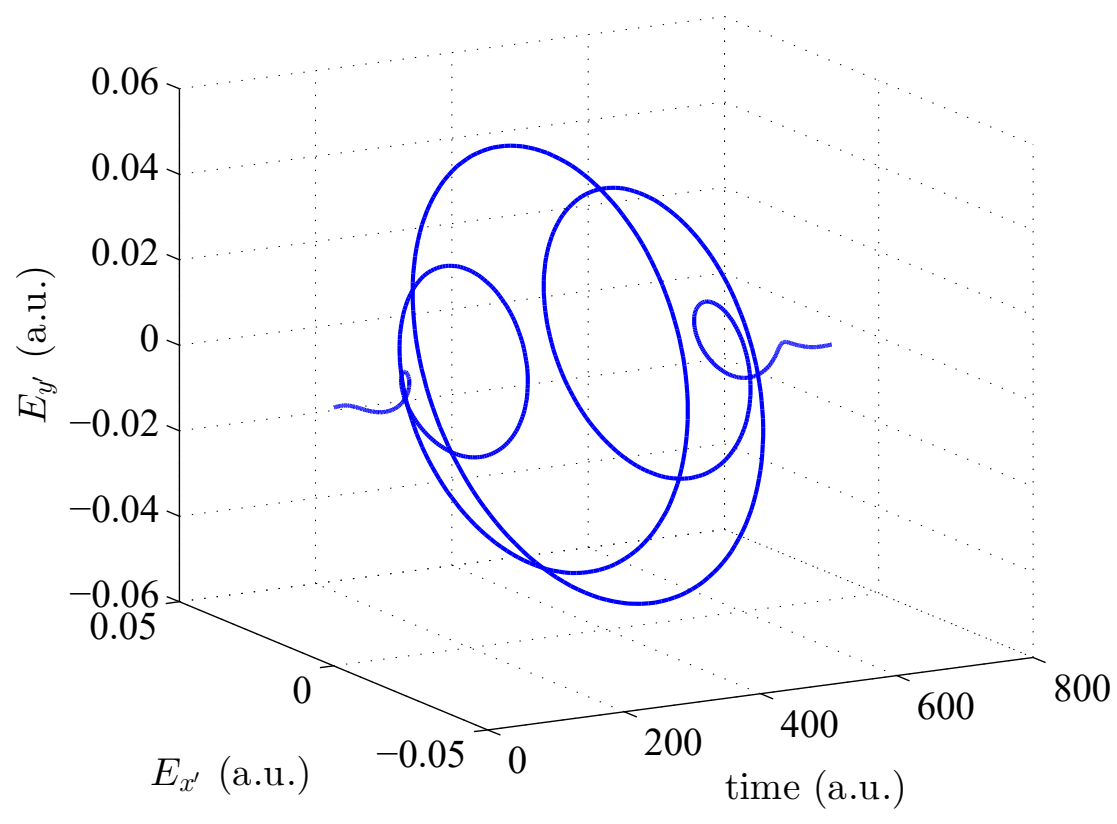

b)

Figure 2.3: Initial CP-laser pulse in the form of Gaussian envelope, with $\alpha=3 \cdot 10^{-9}$ : (a) $E_{x^{\prime}}, E_{y^{\prime}}$ amplitudes of the pulse, with $I=10^{14} \mathrm{~W} / \mathrm{cm}^{2}$, and $\lambda_{0}=800 \mathrm{~nm}$. (b) Total pulse in time. $E$ (a.u.) $=5 \times 10^{9} \mathrm{~V} / \mathrm{cm}$. 
opment of the MASP model, e.g, as proposed in Section 2.3. To this end, we will use another type of wave envelope, which is $f\left(k_{0} z^{\prime}-\omega_{0} t\right)=\sin ^{2}\left[2 \pi N\left(k_{0} z^{\prime}-\omega_{0} t\right)\right]$, where $N$ is desired number of cycles in the pulse. Fig. 2.4 allows to compare both envelopes with equal number of cycles. We can expect that the just introduced $\sin ^{2}$ envelope will work better when studying the spectrum of a pulse passing through the gas region.

Proposition 2.1 (Self-consistency of the 1d-2d MASP model). Assuming that the initial laser pulse is given by

$$
\left\{\begin{array}{l}
\mathbf{E}_{0}\left(z^{\prime}\right)=\left(E_{x^{\prime}}\left(z^{\prime}\right), E_{y^{\prime}}\left(z^{\prime}\right), 0\right)^{T}, \\
\mathbf{B}_{0}\left(z^{\prime}\right)=\left(B_{x^{\prime}}\left(z^{\prime}\right), B_{y^{\prime}}\left(z^{\prime}\right), 0\right)^{T},
\end{array}\right.
$$

and each $\mathrm{H}_{2}^{+}$-molecule lies in the plane $(x, y)$, then for all $t>0$ :

$$
\left\{\begin{array}{l}
\mathbf{E}\left(\mathbf{x}^{\prime}, t\right)=\left(E_{x^{\prime}}\left(z^{\prime}, t\right), E_{y^{\prime}}\left(z^{\prime}, t\right), 0\right)^{T}, \\
\mathbf{B}\left(\mathbf{x}^{\prime}, t\right)=\left(B_{x^{\prime}}\left(z^{\prime}, t\right), B_{y^{\prime}}\left(z^{\prime}, t\right), 0\right)^{T} .
\end{array}\right.
$$

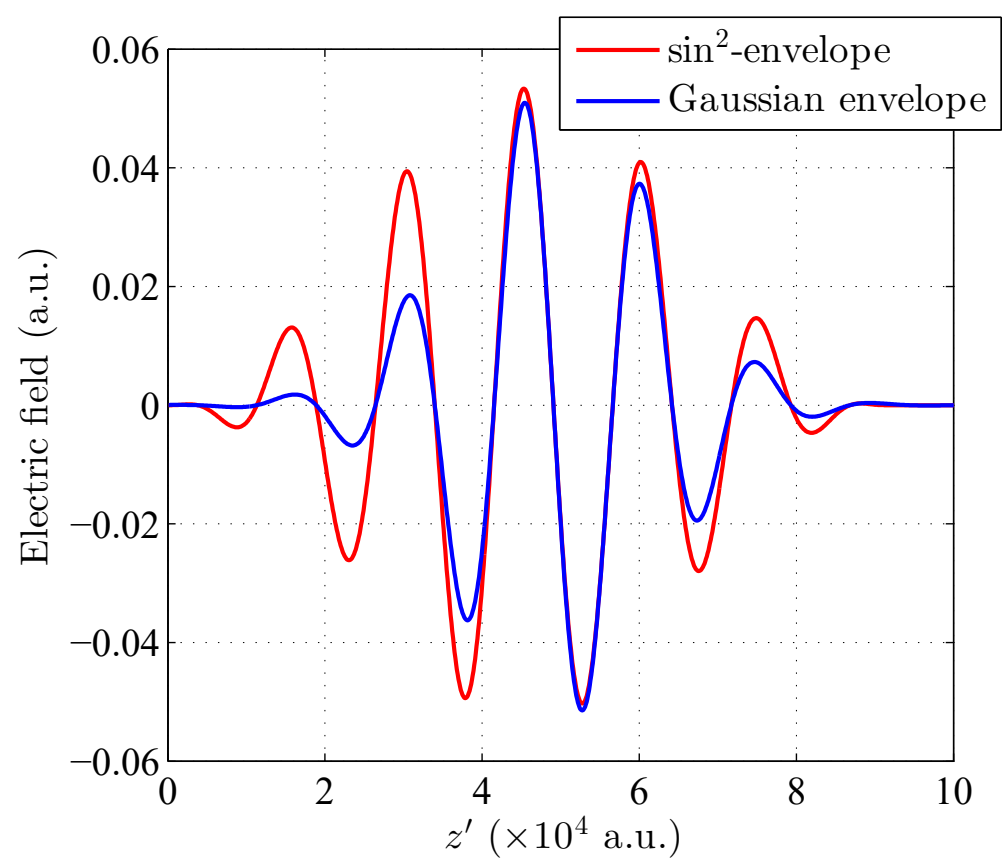

FiguRE 2.4: Initial linearly polarized laser pulses modulated by $\sin ^{2}$ - and Gaussian envelopes with the same number of cycles: $N=6$. 
Proof. If the $\mathrm{H}_{2}^{+}$-molecule lies in the plane $(x, y)$, then its wavefunction $\psi(\mathbf{x}, t)$ is a 2-dimensional function of coordinates: $\mathbf{x}=(x, y)$. As this is true for all molecules, then according to $(2.2)$ and $(2.4) P_{z^{\prime}}\left(z^{\prime}, t\right)=0$ for all $t>0$, and hence $\partial_{t} P_{z^{\prime}}\left(z^{\prime}, t\right) \equiv 0$. In the $1 \mathrm{~d}$ model it follows from (2.9), (2.10) that the free electron number density is a function of the coordinate $z^{\prime}$ and time only: $\mathcal{N}_{e}\left(z^{\prime}, t\right)$. Also in $1 \mathrm{~d}$ model $[\nabla \times$ $\left.\mathbf{B}\left(z^{\prime}, t\right)\right]_{z^{\prime}} \equiv 0$, for all $t>0$, so that we get from (2.1) and (2.5) the following first order ODE system for $E_{z^{\prime}}$ and $J_{z^{\prime}}$ :

$$
\left\{\begin{array}{l}
\partial_{t} E_{z^{\prime}}\left(z^{\prime}, t\right)=-4 \pi J_{z^{\prime}}\left(z^{\prime}, t\right) \\
\partial_{t} J_{z^{\prime}}\left(z^{\prime}, t\right)=\mathcal{N}_{e}\left(z^{\prime}, t\right) E_{z^{\prime}}\left(z^{\prime}, t\right)-\nu_{e} J_{z^{\prime}}\left(z^{\prime}, t\right)
\end{array}\right.
$$

with ICs $E_{z^{\prime}}\left(z^{\prime}, 0\right)=0$ and $J_{z^{\prime}}\left(z^{\prime}, 0\right)=0$. It follows that at $t=0$ the Wronskian of the system $W_{0}=0$. We deduce that $W(t)=W_{0} e^{-\nu_{e} t}$, hence $W(t) \equiv 0$ and the solutions are then linearly dependent. There exists a constant $C \neq 0$, such that $J_{z^{\prime}}\left(z^{\prime}, t\right)=C E_{z^{\prime}}\left(z^{\prime}, t\right)$, for any $t>0$. On the other hand, from $W(t) \equiv 0$ we can conclude that $\mathcal{N}_{e}\left(z^{\prime}, t\right) E_{z^{\prime}}^{2}\left(z^{\prime}, t\right)-\nu_{e} J_{z^{\prime}}\left(z^{\prime}, t\right) E_{z^{\prime}}\left(z^{\prime}, t\right)=-4 \pi J_{z^{\prime}}^{2}\left(z^{\prime}, t\right)$, and

$$
E_{z^{\prime}}^{2}\left(z^{\prime}, t\right)\left(\mathcal{N}_{e}\left(z^{\prime}, t\right)-\nu_{e} C+4 \pi C^{2}\right)=0, \quad \text { for all } t>0
$$

which is possible only if $E_{z^{\prime}}\left(z^{\prime}, t\right) \equiv 0$ (as well as $J_{z^{\prime}}\left(z^{\prime}, t\right) \equiv 0$ ). In other words, the 1d-2d MASP model is self-consistent. In particular, it can be used for describing HHG for CP-laser pulse, see Fig. 2.5.

\section{Gaussian beam: 3d-1d and 3d-2d MASP models.}

For accurate physical description of laser filamentation, we should take into account the dependence of the electric field component(s) on the spatial coordinate(s) transversal to the direction of the pulse propagation (see Section 1.3.2). To this end, we apply the $3 \mathrm{~d}-1 \mathrm{~d} / 2 \mathrm{~d}$ MASP model with coordinates $\mathbf{x}^{\prime}=\left(x^{\prime}, y^{\prime}, z^{\prime}\right)$. For example, it is reasonable to use a Gaussian beam as an initiating laser pulse. In this case, the beam propagates in the $z^{\prime}>0$ direction, with polarization in the $y^{\prime}$ direction. The 


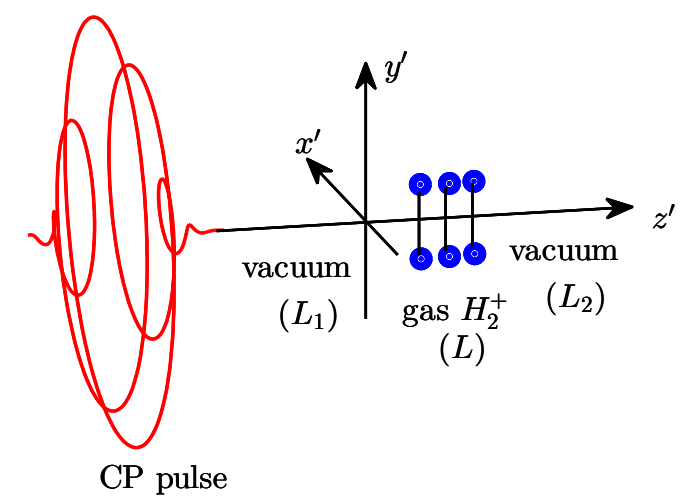

Figure 2.5: 1d-2d geometry of HHG from an incident CP-pulse on aligned $\mathrm{H}_{2}^{+}$-molecules. $L_{1}$ and $L_{2}$ are the lengths of the vacuum regions, $L$ is the length of the $\mathrm{H}_{2}^{+}$-gas region.

electric field then reads

$$
\begin{aligned}
E_{y^{\prime}}\left(x^{\prime}, y^{\prime}, z^{\prime}\right)= & E_{0}\left(\frac{w_{0}}{w\left(z^{\prime}\right)}\right) \exp \left[-\frac{x^{\prime 2}+y^{\prime 2}}{w\left(z^{\prime}\right)^{2}}\right] \exp \left[-\frac{\left(z^{\prime}-z_{0}^{\prime}\right)^{2}}{z_{L}^{\prime 2}}\right] \times \\
& \times \sin \left[\arctan \left(\frac{z^{\prime}}{z_{R}^{\prime}}\right)-\left(\frac{z^{\prime}}{z_{R}^{\prime}}\right) \frac{x^{\prime 2}+y^{\prime 2}}{w\left(z^{\prime}\right)^{2}}-k\left(z^{\prime}-z_{0}^{\prime}\right)\right]
\end{aligned}
$$

where the point $z^{\prime}=0$ corresponds to the location of the waist (focus) of the beam and the point $\left(x^{\prime}, y^{\prime}\right)=(0,0)$ lies on the axis of symmetry of the beam. As in Section 1.3.1, $w_{0}$ denotes the "waist" of the Gaussian beam, see Fig. 1.6, then $w\left(z^{\prime}\right)=$ $w_{0} \sqrt{1+\left(z^{\prime} / z_{R}^{\prime}\right)^{2}}$ is a characteristic radius of the field distribution, where the Rayleigh length is $z_{R}^{\prime}=k w_{0}^{2} / 2$, and wavenumber is $k=\omega / c$. In the above expression for the field, $z_{L}^{\prime}$ is a characteristic length of the beam envelope centred at $z_{0}^{\prime}$, and the amplitude $E_{0}$ of the electric field corresponds to the maximal intensity, $I_{0}=c E_{0}^{2} / 8 \pi$.

Recall that the Gaussian beam defined in (2.13) is not a solution to the MEs in vacuum but an exact solution to the Helmholtz equation (1.70). This implies that we should consider the waist large enough with respect to the wavelength, i.e. $w_{0} \gg 1 / k$ (paraxial approximation), then the initial magnetic field component computes as $B_{x^{\prime}}\left(x^{\prime}, y^{\prime}, z^{\prime}\right)=-E_{y^{\prime}}\left(x^{\prime}, y^{\prime}, z^{\prime}\right)$, which is perpendicular to both: the direction of the beam propagation and the electric field. Moreover, according to the 3d-MEs, the electric and the magnetic fields should have more than one nonzero components. 
Even if initially $E_{x^{\prime}}, E_{z^{\prime}}, B_{y^{\prime}}$ and $B_{z^{\prime}}$ are zeros, but $E_{y^{\prime}}\left(x^{\prime}, y^{\prime}, z^{\prime}\right)$ and $B_{x^{\prime}}\left(x^{\prime}, y^{\prime}, z^{\prime}\right)$ are nonzero, all components become non-zero in time. If we need to be more accurate in our computations, we should also consider $E_{z^{\prime}}$ and $B_{z^{\prime}}$, which at first order of small enough parameter $\varepsilon=1 / k w_{0}$ read [102]:

$$
\begin{aligned}
E_{z^{\prime}}\left(x^{\prime}, y^{\prime}, z^{\prime}\right)= & 2 \varepsilon y^{\prime} E_{0}\left(\frac{w_{0}}{w\left(z^{\prime}\right)^{2}}\right) \exp \left[-\frac{x^{\prime 2}+y^{\prime 2}}{w\left(z^{\prime}\right)^{2}}\right] \exp \left[-\frac{\left(z^{\prime}-z_{0}^{\prime}\right)^{2}}{z_{L}^{\prime 2}}\right] \times \\
& \times \cos \left[2 \arctan \left(\frac{z^{\prime}}{z_{R}^{\prime}}\right)-\left(\frac{z^{\prime}}{z_{R}^{\prime}}\right) \frac{x^{\prime 2}+y^{\prime 2}}{w\left(z^{\prime}\right)^{2}}-k\left(z^{\prime}-z_{0}^{\prime}\right)\right], \\
B_{z^{\prime}}\left(x^{\prime}, y^{\prime}, z^{\prime}\right)= & 2 \varepsilon x^{\prime} E_{0}\left(\frac{w_{0}}{w\left(z^{\prime}\right)^{2}}\right) \exp \left[-\frac{x^{\prime 2}+y^{\prime 2}}{w\left(z^{\prime}\right)^{2}}\right] \exp \left[-\frac{\left(z^{\prime}-z_{0}^{\prime}\right)^{2}}{z_{L}^{\prime 2}}\right] \times \\
& \times \cos \left[2 \arctan \left(\frac{z^{\prime}}{z_{R}^{\prime}}\right)-\left(\frac{z^{\prime}}{z_{R}^{\prime}}\right) \frac{x^{\prime 2}+y^{\prime 2}}{w\left(z^{\prime}\right)^{2}}-k\left(z^{\prime}-z_{0}^{\prime}\right)\right],
\end{aligned}
$$

while $E_{x^{\prime}}$ and $B_{y^{\prime}}$ are zeros at the same order of accuracy. Indeed, since we expect that beam to become narrower, as a result of nonlinear self-action, the above approximation for the fields looks suitable for our purpose.

\subsection{Polarization Evolution Equation for Ultrashort Pulses}

The objective of this section is to show how to considerably reduce the computational complexity of the MASP model. A macroscopic wave equation modeling the evolution of the polarization vector, is derived and coupled to the MEs. Although the model still requires the solutions to TDSEs for evaluating the polarization at some preselected spatial points, this approach allows to reduce drastically the number of TDSEs involved in the model. The simplest polarization evolution equation is a homogeneous transport equation. Then we are proposing more accurate models, including the nonlinear variations of the electric field [83,84], as well as the effect of the free electron current. 


\subsubsection{Simple Evolution Equation for the Dipole Moment}

Within the 1d-2d MASP model, the laser-molecule interactions require the solution of 2d-TDSEs:

$$
\mathrm{i} \partial_{t} \psi_{\mathbf{x}^{\prime}}=-\frac{1}{2} \triangle \psi_{\mathbf{x}^{\prime}}+V_{C}(\mathbf{x}) \psi_{\mathbf{x}^{\prime}}+\mathbf{x} \cdot \mathbf{E}_{\mathbf{x}^{\prime}}(t) \psi_{\mathbf{x}^{\prime}}
$$

with $\mathbf{x}=(x, y), \mathbf{E}_{\mathbf{x}^{\prime}}=\left(E_{x^{\prime}}, E_{y^{\prime}}\right)$. The computation of the wavefunction $\psi_{\mathbf{x}^{\prime}}$ of a molecule "located in $\mathbf{x}^{\prime \prime}$ " allows to deduce the dipole moment $\mathbf{d}$ as

$$
\mathbf{d}\left(\mathbf{x}^{\prime}, t\right)=\int_{\mathbb{R}^{2}}\left|\psi_{\mathbf{x}^{\prime}}(\mathbf{x}, t)\right|^{2} \mathbf{x} d x d y
$$

Assuming that the wavefunction, $\psi_{1}$, of Molecule $m_{1}$ "located" in $\mathbf{x}_{1}^{\prime}=\left(x_{1}^{\prime}, y_{1}^{\prime}, z_{1}^{\prime}\right)$ is known, an evolution equation to estimate the dipole moment $\mathbf{d}$ of Molecule $m_{2}$ "located" in $\mathbf{x}_{2}^{\prime}=\left(x_{1}^{\prime}, y_{1}^{\prime}, z_{2}^{\prime}\right)$ with $z_{2}^{\prime}>z_{1}^{\prime}$, can be derived as follows, see also $[77,96]$.

If $\left|\Delta z^{\prime}\right|:=\left|z_{2}^{\prime}-z_{1}^{\prime}\right|$ is small enough to neglect the medium effects on $\mathbf{E}$, we may assume that an electromagnetic field interacting with Molecule $m_{2}$ is almost identical (up to a time delay) to the one that Molecule $m_{1}$ was subject to. Note that overall, we do not assume that the electromagnetic field propagates as in a linear medium (or vacuum) in Maxwell's equations. Let us define $\psi_{i}$ the wavefunction of Molecule $m_{i}$

$$
i \partial_{t} \psi_{i}=-\frac{1}{2} \triangle \psi_{i}+V_{C}(\mathbf{x}) \psi_{i}+\mathbf{x} \cdot \mathbf{E}_{i}(t) \psi_{i} .
$$

and $\mathbf{d}\left(\mathbf{x}_{i}^{\prime}, t\right)$ the corresponding dipole moment, with $i=1,2$. In addition $\mathbf{E}_{i}(t)=$ $\mathbf{E}\left(\mathbf{x}_{i}^{\prime}, t\right)$ denotes the electric field that Molecule $m_{i}$ is subject to. The above assumption mathematically implies: $\mathbf{E}_{2}(t)=\mathbf{E}_{1}\left(t-\Delta z^{\prime} / v_{g}\right)$ and as a consequence

$$
\mathbf{d}\left(\mathbf{x}_{2}^{\prime}, t\right)=\mathbf{d}\left(\mathbf{x}_{1}^{\prime}, t-\frac{\Delta z^{\prime}}{v_{g}}\right)
$$




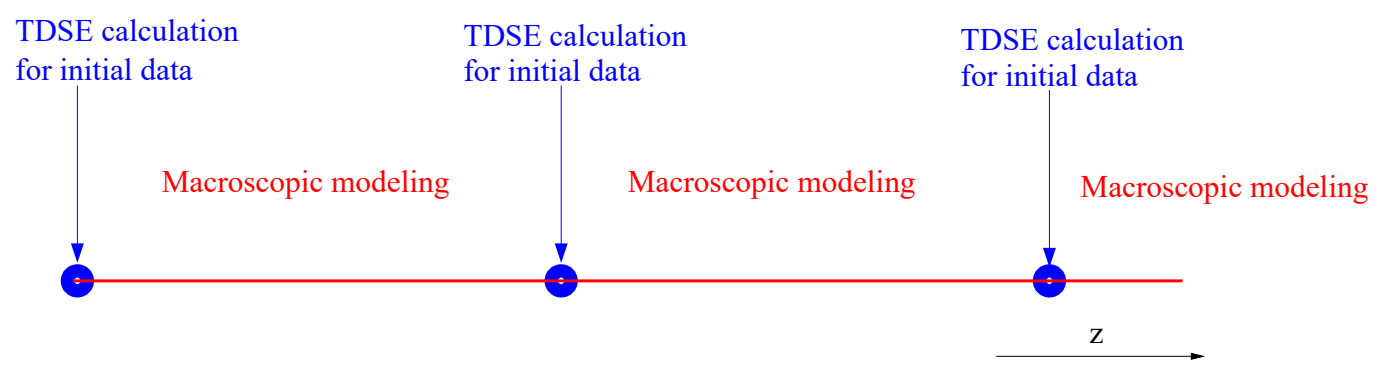

FiguRE 2.6: Macroscopic model with initial data from TDSE [84].

where $v_{g}$ is the group velocity ( $c$ in vacuum). Then the polarization $\mathbf{P}$ satisfies for $z^{\prime} \in\left[z_{1}^{\prime}, z_{2}^{\prime}\right]$, the following transport equation

\section{$\triangleright$ Simple evolution equation}

$$
\partial_{t} \mathbf{P}\left(\mathbf{x}^{\prime}, t\right)+v_{g} \partial_{z^{\prime}} \mathbf{P}\left(\mathbf{x}^{\prime}, t\right)=\mathbf{0}
$$

with initial data $\mathbf{P}\left(\mathbf{x}_{1}^{\prime}, 0\right)=\mathcal{N}_{0} \mathbf{d}\left(\mathbf{x}_{1}^{\prime}, 0\right)$. The model is then purely macroscopic (except for the computation of the initial data for $\mathbf{P}$ ), see Fig 2.6. This model is applicable as long as $\left|\Delta z^{\prime}\right|$ is small enough, or/and if the molecule density is small enough, that is, as long as the effect of the medium on $\mathbf{E}$ during the pulse propagation from $\mathbf{x}_{1}^{\prime}$ to $\mathbf{x}_{2}^{\prime}$ is sufficiently negligible as not to be included in the dipole moment calculation of Molecule $m_{2}$. In order to include the medium effects on $\mathbf{E}$ in the propagation from $\mathbf{x}_{1}^{\prime}$ to $\mathbf{x}_{2}^{\prime}$, an improvement of the model is then necessary. In particular, this approach developed below will allow to consider larger propagation lengths and times.

First, in order to estimate the group velocity $v_{g}$ for (2.17) in a given medium for which $n_{0}$ and $n_{2}$ are approximately known (through $\chi^{(1)}$ and $\left.\chi^{(3)}\right)$, we use the standard approximation $n(\omega) \approx n_{0}(\omega)+n_{2}(\omega) I$, see (1.62). By definition, the group velocity is given by $v_{g}=\partial_{k} \omega$, where $k=k_{z^{\prime}}$, while the phase velocity $v_{p h}=\frac{\omega}{k}=\frac{c}{n}$, resulting in $v_{g}=\frac{c}{n(\omega)+\omega \partial_{\omega} n(\omega)}$. In first approximation, $n(\omega) \approx n_{0}=\sqrt{\epsilon^{(1)}}=\sqrt{1+4 \pi \chi^{(1)}}$, so that we approximately have 


$$
v_{g} \approx \frac{c}{\sqrt{1+4 \pi \chi^{(1)}}}
$$

where $\chi^{(1)}$ is the instantaneous linear susceptibility.

The evolution equation for $\mathbf{P}$ (2.17) is then coupled to MEs (2.1) for modeling the propagation of the laser pulse over $\left[z_{1}^{\prime}, z_{2}^{\prime}\right]$. Polarization at $\left(x^{\prime}, y^{\prime}, z^{\prime}\right)$ for $z^{\prime}>z_{2}^{\prime}$, is then computed again using (2.2). More specifically, the domain is decomposed in subdomains in the $z^{\prime}$ direction, and TDSEs are computed only at preselected locations of each sudomain, in order to evaluate the dipole moment in the corresponding Maxwell's cell. Between these points, the macroscopic evolution equations on $\mathbf{P}$ are

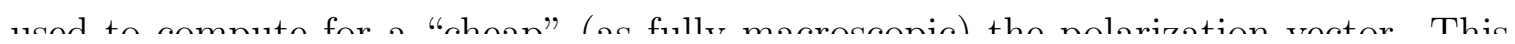

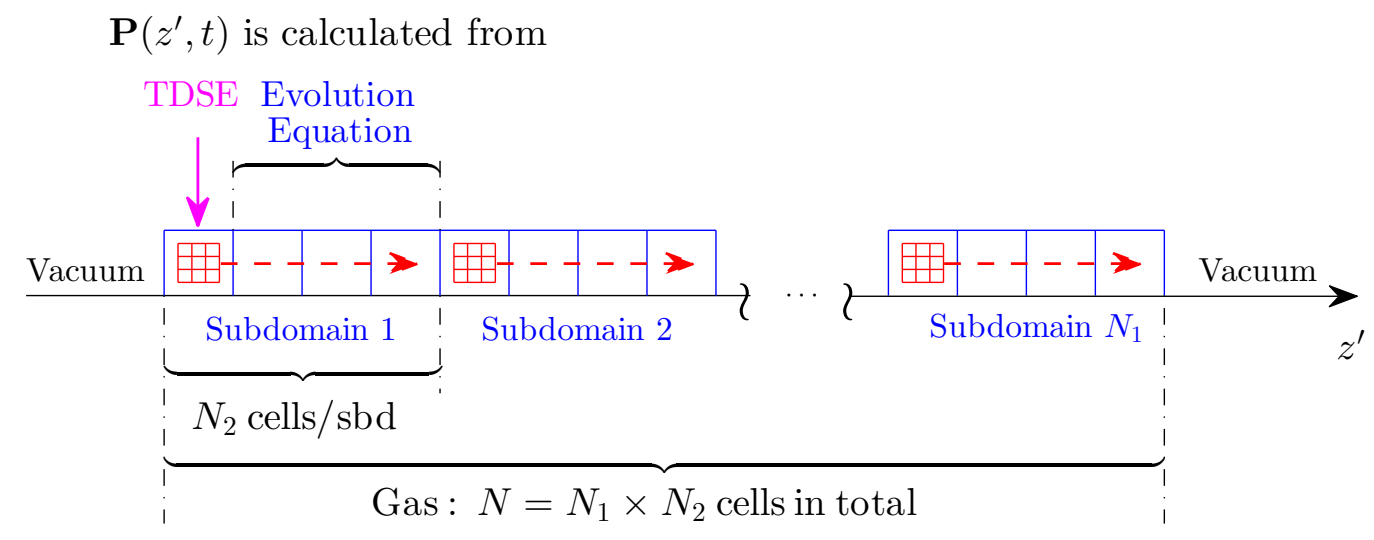

Figure 2.7: Spatial evolution of polarization. Macroscopic model with initial data computed from TDSE. The computational domain is divided along $z^{\prime}$ into $N_{1}$ subdomains containing $N_{2}$ Maxwell's cells, length $h$ each, so that the total propagation path in the gas is $L=N_{1} \times N_{2} \times h$ units of length. 


\subsubsection{General Evolution Equation for the Dipole Moment}

Since we consider multidimensional EM field propagation, including forward and backward propagation effects, the polarization equation is expected to be of the form:

$$
\partial_{t}^{2} \mathbf{P}-v_{g}^{2} \triangle \mathbf{P}=\mathbf{S}(\mathbf{E})
$$

with initial data to be determined from quantum TDSEs, and where for brevity sake we denote $\mathbf{P} \equiv \mathbf{P}\left(\mathbf{x}^{\prime}, t\right), \mathbf{E} \equiv \mathbf{E}\left(\mathbf{x}^{\prime}, t\right)$. The main idea consists of including as much information as possible to determine $\mathbf{S}(\mathbf{E})$ in (2.19).

We begin from the 1st and 2nd MEs (2.1) written for nonmagnetic medium, $\mathbf{B}=\mathbf{H}$ :

$$
\left\{\begin{array}{l}
c \nabla \times \mathbf{B}=\partial_{t} \mathbf{E}+4 \pi \partial_{t} \mathbf{P}+4 \pi \mathbf{J}, \\
c \nabla \times \mathbf{E}=-\partial_{t} \mathbf{B}
\end{array}\right.
$$

Excluding $\mathbf{B}$ in the same manner as in (1.32)-(1.36), we get the wave equation for $\mathbf{E}$ :

$$
\partial_{t}^{2} \mathbf{E}-c^{2} \triangle \mathbf{E}+c^{2} \nabla(\nabla \cdot \mathbf{E})=-4 \pi\left(\partial_{t}^{2} \mathbf{P}+\partial_{t} \mathbf{J}\right) .
$$

Supposing that on the relevant propagation length nonlinearity is not very strong (recall that $\nabla \cdot \mathbf{E} \propto \nabla \cdot \mathbf{P}^{N L}$, see (1.35)), we can as usual omit the term $\nabla(\nabla \cdot \mathbf{E})[24]$ :

$$
\partial_{t}^{2} \mathbf{E}-c^{2} \triangle \mathbf{E}=-4 \pi\left(\partial_{t}^{2} \mathbf{P}+\partial_{t} \mathbf{J}\right) .
$$

Next, we assume that the perturbative expansion

$$
\mathbf{P}\left(\mathbf{x}^{\prime}, t\right)=\chi^{(1)} \mathbf{E}\left(\mathbf{x}^{\prime}, t\right)+\chi^{(3)}\left(\mathbf{E}\left(\mathbf{x}^{\prime}, t\right) \cdot \mathbf{E}\left(\mathbf{x}^{\prime}, t\right)\right) \mathbf{E}\left(\mathbf{x}^{\prime}, t\right),
$$

where $\chi^{(1)}$ and $\chi^{(3)}$ are the first and third instantaneous susceptibilities of isotropic medium, see (1.3)-(1.5), is an accurate approximation for $\mathbf{P}$ at least for a certain 
propagation length. From here

$$
\mathbf{E}\left(\mathbf{x}^{\prime}, t\right)=\frac{1}{\chi^{(1)}} \mathbf{P}\left(\mathbf{x}^{\prime}, t\right)-\frac{\chi^{(3)}}{\chi^{(1)}}\left(\mathbf{E}\left(\mathbf{x}^{\prime}, t\right) \cdot \mathbf{E}\left(\mathbf{x}^{\prime}, t\right)\right) \mathbf{E}\left(\mathbf{x}^{\prime}, t\right)
$$

Then substituting (2.24) in (2.22) leads to

$$
\left(1+4 \pi \chi^{(1)}\right) \partial_{t}^{2} \mathbf{P}-c^{2} \triangle \mathbf{P}=\chi^{(3)}\left[\partial_{t}^{2}(\mathbf{E}(\mathbf{E} \cdot \mathbf{E}))-c^{2} \triangle(\mathbf{E}(\mathbf{E} \cdot \mathbf{E}))\right]-4 \pi \chi^{(1)} \partial_{t} \mathbf{J},
$$

where $(\mathbf{E} \cdot \mathbf{E}) \equiv|\mathbf{E}|^{2}=E_{x}^{2}+E_{y}^{2}+E_{z}^{2}$.

Recall that the group velocity was defined in (2.18) as $v_{g}=\frac{c}{\sqrt{1+4 \pi \chi^{(1)}}}$, so that the evolution equation for $\mathbf{P}$ can be shaped into

$$
\partial_{t}^{2} \mathbf{P}-v_{g}^{2} \triangle \mathbf{P}=\left(\frac{v_{g}}{c}\right)^{2}\left\{\chi^{(3)}\left[\partial_{t}^{2}\left(\mathbf{E E}^{2}\right)-c^{2} \triangle\left(\mathbf{E} \mathbf{E}^{2}\right)\right]-4 \pi \chi^{(1)} \partial_{t} \mathbf{J}\right\}
$$

At preselected locations, say $\mathbf{x}_{\alpha}^{\prime}$ and at each computational time step $t_{\beta}$, the data $\mathbf{P}\left(\mathbf{x}_{\alpha}^{\prime}, t_{\beta}\right), \partial_{t} \mathbf{P}\left(\mathbf{x}_{\alpha}^{\prime}, t_{\beta}\right)$ are computed from microscopic TDSEs:

$$
\left\{\begin{array}{l}
\mathbf{P}\left(\mathbf{x}_{\alpha}^{\prime}, t_{\beta}\right)=\mathcal{N}\left(\mathbf{x}_{\alpha}^{\prime}\right) \int_{\mathbb{R}^{3}}\left|\psi_{\mathbf{x}_{\alpha}^{\prime}}\left(\mathbf{x}, t_{\beta}\right)\right|^{2} \mathbf{x} d^{3} \mathbf{x} \\
\partial_{t} \mathbf{P}\left(\mathbf{x}_{\alpha}^{\prime}, t_{\beta}\right)=\mathcal{N}\left(\mathbf{x}_{\alpha}^{\prime}\right) \int_{\mathbb{R}^{3}} \partial_{t}\left|\psi_{\mathbf{x}_{\alpha}^{\prime}}\left(\mathbf{x}, t_{\beta}\right)\right|^{2} \mathbf{x} d^{3} \mathbf{x}
\end{array}\right.
$$

while the current density $\mathbf{J}$ satisfies the macroscopic evolution equation, see (2.5):

$$
\partial_{t} \mathbf{J}+\nu_{e} \mathbf{J}=\mathcal{N}_{e} \mathbf{E}
$$

Note that the free electron number density in the last equation is assumed to be a function of $z^{\prime}$-coordinate and time. It is reasonable to compute this value from the corresponding transport equation:

$$
\partial_{t} \mathcal{N}_{e}+v_{g} \partial_{z^{\prime}} \mathcal{N}_{e}=0
$$


with the initial distribution computed in accordance with (2.9) and (2.10):

$$
\mathcal{N}_{e}\left(z_{\alpha}^{\prime}, t_{\beta}\right)=\mathcal{N}_{e 0}\left(z_{\alpha}^{\prime}\right)+\mathcal{N}\left(z_{\alpha}^{\prime}\right) \sum_{i=1}^{m} \chi_{\Omega_{i}}\left(z_{\alpha}^{\prime}\right)\left[1-\int_{\Omega_{i}}\left|\psi_{i}\left(\mathbf{x}, t_{\beta}\right)\right|^{2} d^{3} \mathbf{x}\right]
$$

Thus, except for the evaluation of the polarization and the free electron number density at the specific points, requiring TDSE computations, the evolution equations (2.26) and (2.29) are then fully macroscopic.

It is necessary to comment the philosophy of the proposed approach. Equations (2.26), (2.27) form a nonperturbative model, which includes (i) the transport of the nonperturbative data $\left(\partial_{t} \mathbf{P}_{0}\right.$ is computed from the TDSE) from the left boundary to the right one of each subdomain, and (ii) the generation of perturbative nonlinearities within these subdomains. As we mentioned in Section 2.3.1, in case (2.17) we do not assume propagation in a linear medium, however, we use a linear approximation that is valid when $\Delta z^{\prime}$ is short enough, and the same was assumed while deriving (2.26) from (2.22)-(2.24). Thus, in our model approach the LHS in the equation (2.26) represents the linear transport of nonperturbative polarization with reference points $\partial_{t} \mathbf{P}\left(\mathbf{x}_{\alpha}^{\prime}, \cdot\right), \alpha=1, \ldots, N_{2}$ and $\Delta z^{\prime}=\left|\mathbf{x}_{\alpha}^{\prime}-\mathbf{x}_{\alpha-1}^{\prime}\right|$, taken from the nonperturbative computations (2.27). The RHS of the equation (2.26) makes the first non-zero perturbative correction for the interval $\Delta z^{\prime}$. We demonstrate the results of this approach in Section 4.2.2.

For practical purposes, we simplify the polarization evolution equation in the case of $1 \mathrm{~d}-2 \mathrm{~d}$ model, i.e. supposing that $\mathbf{P}=\left(P_{x^{\prime}}\left(z^{\prime}, t\right), P_{y^{\prime}}\left(z^{\prime}, t\right)\right)^{T}$. We start with the case of LP-pulse, implying that $P_{x^{\prime}}\left(z^{\prime}, t\right)$ as well as $E_{x^{\prime}}\left(z^{\prime}, t\right)$ are null.

Thus, taking into account that

$$
\partial_{t}^{2}(\mathbf{E}(\mathbf{E} \cdot \mathbf{E}))=4 \partial_{t} \mathbf{E}\left(\mathbf{E} \cdot \partial_{t} \mathbf{E}\right)+2 \mathbf{E}\left(\partial_{t} \mathbf{E}\right)^{2}+\mathbf{E}^{2} \partial_{t}^{2} \mathbf{E}+2 \mathbf{E}\left(\partial_{t}^{2} \mathbf{E} \cdot \mathbf{E}\right)
$$


and assuming that the polarization vector propagates along the $z^{\prime}$-direction $(\triangle$ is reduced to $\partial_{z^{\prime}}^{2}$ ) we obtain an evolution equation for $P_{y^{\prime}}$ :

$$
\begin{aligned}
\partial_{t}^{2} P_{y^{\prime}}- & v_{g}^{2} \partial_{z^{\prime}}^{2} P_{y^{\prime}}=3 \chi^{(3)}\left(\frac{v_{g}}{c}\right)^{2} E_{y^{\prime}}^{2}\left[\partial_{t}^{2} E_{y^{\prime}}-c^{2} \partial_{z^{\prime}}^{2} E_{y^{\prime}}\right]+ \\
& +6 \chi^{(3)}\left(\frac{v_{g}}{c}\right)^{2} E_{y^{\prime}}\left[\left(\partial_{t} E_{y^{\prime}}\right)^{2}-c^{2}\left(\partial_{z^{\prime}} E_{y^{\prime}}\right)^{2}\right]-4 \pi \chi^{(1)}\left(\frac{v_{g}}{c}\right)^{2} \partial_{t} J_{y^{\prime}}
\end{aligned}
$$

It follows from (2.22) that in $1 \mathrm{~d} \partial_{t}^{2} E_{y^{\prime}}-c^{2} \partial_{z^{\prime}}^{2} E_{y^{\prime}}=-4 \pi \partial_{t}^{2} P_{y^{\prime}}-4 \pi \partial_{t} J_{y^{\prime}}$, so that

$$
\begin{aligned}
& \left(1+12 \pi \chi^{(3)}\left(\frac{v_{g}}{c}\right)^{2} E_{y^{\prime}}^{2}\right) \partial_{t}^{2} P_{y^{\prime}}-v_{g}^{2} \partial_{z}^{2} P_{y^{\prime}}= \\
& =6 \chi^{(3)}\left(\frac{v_{g}}{c}\right)^{2} E_{y^{\prime}}\left[\left(\partial_{t} E_{y^{\prime}}\right)^{2}-c^{2}\left(\partial_{z} E_{y^{\prime}}\right)^{2}\right]-4 \pi\left(\frac{v_{g}}{c}\right)^{2}\left(\chi^{(1)}+3 \chi^{(3)} E_{y^{\prime}}^{2}\right) \partial_{t} J_{y^{\prime}}
\end{aligned}
$$

For the sake of simplicity we introduce three variable factors

$$
\begin{aligned}
a\left(E_{y^{\prime}}\left(z^{\prime}, t\right)\right) & :=\left(1+12 \pi \chi^{(3)}\left(v_{g} / c\right)^{2} E_{y^{\prime}}^{2}\right)^{-1}, \\
b\left(E_{y^{\prime}}\left(z^{\prime}, t\right)\right) & :=6 \chi^{(3)}\left(v_{g} / c\right)^{2} E_{y^{\prime}} \\
g\left(E_{y^{\prime}}\left(z^{\prime}, t\right)\right) & :=4 \pi\left(\frac{v_{g}}{c}\right)^{2}\left(\chi^{(1)}+3 \chi^{(3)} E_{y^{\prime}}^{2}\right),
\end{aligned}
$$

so that the polarization wave equation takes the form

$$
\partial_{t}^{2} P_{y^{\prime}}-a\left(E_{y^{\prime}}\right) v_{g}^{2} \partial_{z^{\prime}} P_{y^{\prime}}=a\left(E_{y^{\prime}}\right) b\left(E_{y^{\prime}}\right)\left[\left(\partial_{t} E_{y^{\prime}}\right)^{2}-c^{2}\left(\partial_{z} E_{y^{\prime}}\right)^{2}\right]-a\left(E_{y^{\prime}}\right) g\left(E_{y^{\prime}}\right) \partial_{t} J_{y^{\prime}}
$$

Recall that we have in mind to couple this equation with the MASP model, in other words, the numerical solution of this equation is referred to time $t^{n *} \in\left[t^{n}, t^{n+1}\right]$ (see Section 3.6 for details). However, the RHS of (2.35) contains partial derivative of the electric field in time, and this fact may result in computational instability. To avoid possible instability of the solution, we replace the partial derivative $\partial_{t} E_{y^{\prime}}$ in $(2.35)$ in accordance with the MEs: $\partial_{t} E_{y^{\prime}}=c \partial_{z^{\prime}} B_{x^{\prime}}-4 \pi \partial_{t} P_{y^{\prime}}-4 \pi J_{y^{\prime}}$, so that the resulting nonlinear PDE reads: 


\section{$\triangleright$ Evolution equation for 1d-2d model, LP-pulse}

$$
\begin{gathered}
\partial_{t}^{2} P_{y^{\prime}}-a\left(E_{y^{\prime}}\right) v_{g}^{2} \partial_{z^{\prime}}^{2} P_{y^{\prime}}+8 \pi a\left(E_{y^{\prime}}\right) b\left(E_{y^{\prime}}\right)\left[\left(c \partial_{z} B_{x^{\prime}}-4 \pi J_{y^{\prime}}\right)\left(\partial_{t} P_{y^{\prime}}\right)-2 \pi\left(\partial_{t} P_{y^{\prime}}\right)^{2}\right]= \\
=a\left(E_{y^{\prime}}\right) b\left(E_{y^{\prime}}\right)\left\{c^{2}\left[\left(\partial_{z^{\prime}} B_{x^{\prime}}\right)^{2}-\left(\partial_{z} E_{y^{\prime}}\right)^{2}\right]+8 \pi J_{y^{\prime}}\left[2 \pi J_{y^{\prime}}-c \partial_{z^{\prime}} B_{x^{\prime}}\right]\right\}- \\
-a\left(E_{y^{\prime}}\right) g\left(E_{y^{\prime}}\right) \partial_{t} J_{y^{\prime}}
\end{gathered}
$$

Then this equation should be coupled to the MASP model as described in Section 2.3.1 for homogeneous transport equation (2.17). Here we give the principal details of the coupling procedure.

- At the time $t=t_{\beta}$, the derivatives in time of polarization $\partial_{t} P_{y^{\prime}}\left(z_{\alpha, 1}^{\prime}, t_{\beta}\right)$ are computed at mesh nodes $\left\{z_{\alpha, 1}^{\prime}\right\}\left(\alpha=1, \ldots N_{1}\right)$ from the set of TDSEs according to (2.27), see Fig. 2.7;

- These data are used as boundary conditions for the wave equation (2.36) with $\partial_{z^{\prime}} B_{x^{\prime}}\left(z^{\prime}, t_{\beta}\right)$ and $\partial_{z^{\prime}} E_{y^{\prime}}\left(z^{\prime}, t_{\beta}\right)$ in the RHS, in order to compute the time derivative of polarization $\partial_{t} P_{y^{\prime}}\left(z_{\alpha, \mu}^{\prime}, t_{\beta+1}\right)\left(\alpha=1, \ldots N_{1}, \mu=2 \ldots N_{2}\right)$ at the next time, $t_{\beta+1}$ at all other points $\left\{z_{\alpha, \mu}^{\prime}\right\}$ of the MEs domain;

- Thus, at time $t_{\beta+1}$ we have $\partial_{t} P_{y^{\prime}}\left(z_{\alpha, 1}^{\prime}, t_{\beta+1}\right)$, computed using the TDSEs, and $\partial_{t} P_{y^{\prime}}\left(z_{\alpha, \mu}^{\prime}, t_{\beta+1}\right)\left(\mu=2 \ldots N_{2}\right)$, computed using the polarization evolution equation. We use these data to solve the MEs in all cells of the domain at time $t_{\beta+1}$.

Note that in the case of LP-pulses, it is easy to generalize (2.34) including higher order nonlinearities:

$$
\begin{aligned}
a\left(E_{y^{\prime}}\right) & :=\left[1+4 \pi\left(v_{g} / c\right)^{2}\left(3 \chi^{(3)} E_{y^{\prime}}^{2}+5 \chi^{(5)} E_{y^{\prime}}^{4}+7 \chi^{(7)} E_{y^{\prime}}^{6}+\ldots\right)\right]^{-1}, \\
b\left(E_{y^{\prime}}\right) & :=E_{y^{\prime}}\left(v_{g} / c\right)^{2}\left(6 \chi^{(3)}+20 \chi^{(5)} E_{y^{\prime}}^{2}+42 \chi^{(7)} E_{y^{\prime}}^{4}+\ldots\right) \\
g\left(E_{y^{\prime}}\right) & :=4 \pi\left(v_{g} / c\right)^{2}\left(\chi^{(1)}+3 \chi^{(3)} E_{y^{\prime}}^{2}+5 \chi^{(5)} E_{y^{\prime}}^{4}+7 \chi^{(7)} E_{y^{\prime}}^{6}+\ldots\right)= \\
& =4 \pi\left(v_{g} / c\right)^{2} \chi^{(1)}+\left[a\left(E_{y^{\prime}}\right)\right]^{-1}-1 .
\end{aligned}
$$


Further, we represent the system of evolution equations for $P_{x^{\prime}}\left(z^{\prime}, t\right)$ and $P_{y^{\prime}}\left(z^{\prime}, t\right)$ derived in the case of the initial CP-pulse in the $1 \mathrm{~d}$-2d model for $\mathbf{E}=\left(E_{x^{\prime}}, E_{y^{\prime}}\right)^{T}$ :

\section{$\triangleright$ Evolution equations for 1d-2d models, CP-pulse}

$$
\left\{\begin{array}{c}
\partial_{t}^{2} P_{x^{\prime}}+a_{1} b_{12} \partial_{t}^{2} P_{y^{\prime}}-a_{1} v_{g}^{2} \partial_{z^{\prime}}^{2} P_{x^{\prime}}= \\
=a_{1} b_{1}\left\{6\left[\left(\partial_{t} E_{x^{\prime}}\right)^{2}-c^{2}\left(\partial_{z^{\prime}} E_{x^{\prime}}\right)^{2}\right]+2\left[\left(\partial_{t} E_{y^{\prime}}\right)^{2}-c^{2}\left(\partial_{z^{\prime}} E_{y^{\prime}}\right)^{2}\right]\right\} \\
+4 a_{1} b_{2}\left\{\left(\partial_{t} E_{x^{\prime}}\right)\left(\partial_{t} E_{y^{\prime}}\right)-c^{2}\left(\partial_{z^{\prime}} E_{x^{\prime}}\right)\left(\partial_{z^{\prime}} E_{y^{\prime}}\right)\right\} \\
\partial_{t}^{2} P_{y^{\prime}}+a_{2} b_{12} \partial_{t}^{2} P_{x^{\prime}}-a_{2} v_{g}^{2} \partial_{z^{\prime}}^{2} P_{y^{\prime}}= \\
=a_{2} b_{2}\left\{6\left[\left(\partial_{t} E_{y^{\prime}}\right)^{2}-c^{2}\left(\partial_{z^{\prime}} E_{y^{\prime}}\right)^{2}\right]+2\left[\left(\partial_{t} E_{x^{\prime}}\right)^{2}-c^{2}\left(\partial_{z^{\prime}} E_{x^{\prime}}\right)^{2}\right]\right\} \\
+4 a_{2} b_{1}\left\{\left(\partial_{t} E_{x^{\prime}}\right)\left(\partial_{t} E_{y^{\prime}}\right)-c^{2}\left(\partial_{z^{\prime}} E_{x^{\prime}}\right)\left(\partial_{z^{\prime}} E_{y^{\prime}}\right)\right\}
\end{array}\right.
$$

where the energy-dependent factors $a_{1}, a_{2}, b_{12}, b_{1}$, and $b_{2}$ are defined as

$$
\begin{aligned}
& a_{1} \equiv a_{1}\left(E_{x^{\prime}}, E_{y^{\prime}}\right)=\left(1+4 \pi \chi^{(3)}\left(v_{g} / c\right)^{2}\left(3 E_{x^{\prime}}^{2}+E_{y^{\prime}}^{2}\right)\right)^{-1}, \\
& a_{2} \equiv a_{2}\left(E_{x^{\prime}}, E_{y^{\prime}}\right)=\left(1+4 \pi \chi^{(3)}\left(v_{g} / c\right)^{2}\left(E_{x^{\prime}}^{2}+3 E_{y^{\prime}}^{2}\right)\right)^{-1}, \\
& b_{12} \equiv b_{0}\left(E_{x^{\prime}}, E_{y^{\prime}}\right)=8 \pi \chi^{(3)}\left(v_{g} / c\right)^{2} E_{x^{\prime}} E_{y^{\prime}}, \\
& b_{1} \equiv b_{1}\left(E_{x^{\prime}}\right)=\chi^{(3)}\left(v_{g} / c\right)^{2} E_{x^{\prime}}, \\
& b_{2} \equiv b_{2}\left(E_{y^{\prime}}\right)=\chi^{(3)}\left(v_{g} / c\right)^{2} E_{y^{\prime}} .
\end{aligned}
$$

The system (2.38) can be coupled to the Maxwell-Schrödinger model in the same way as in the case of LP-pulses.

Finally, we discuss the $3 \mathrm{~d}-2 \mathrm{~d}$ models. The system becomes too complicated if we want to take into account the propagation of $P_{x^{\prime}}\left(x^{\prime}, y^{\prime}, z^{\prime}, y\right)$ and $P_{y^{\prime}}\left(x^{\prime}, y^{\prime}, z^{\prime}, t\right)$ consistently with the general equation (2.26). However, if we consider the diffraction effects in the $z^{\prime}$-direction only, by setting $\triangle=\partial_{z^{\prime}}^{2}$, a system similar to (2.38) reads 


\section{$\triangleright$ Evolution equations for 3d-2d models, CP-pulse}

$$
\left\{\begin{array}{l}
\partial_{t}^{2} P_{x^{\prime}}+a_{1} b_{12} \partial_{t}^{2} P_{y^{\prime}}-a_{1} v_{g}^{2} \partial_{z^{\prime}}^{2} P_{x^{\prime}}= \\
=a_{1} b_{1}\left\{6\left[\left(\partial_{t} E_{x^{\prime}}\right)^{2}-c^{2}\left(\partial_{z^{\prime}} E_{x^{\prime}}\right)^{2}\right]+2\left[\left(\partial_{t} E_{y^{\prime}}\right)^{2}-c^{2}\left(\partial_{z^{\prime}} E_{y^{\prime}}\right)^{2}\right]+\right. \\
\left.+2\left[\left(\partial_{t} E_{z^{\prime}}\right)^{2}-c^{2}\left(\partial_{z^{\prime}} E_{z^{\prime}}\right)^{2}\right]\right\}+4 a_{1} b_{2}\left\{\left(\partial_{t} E_{x^{\prime}}\right)\left(\partial_{t} E_{y^{\prime}}\right)-c^{2}\left(\partial_{z^{\prime}} E_{x^{\prime}}\right)\left(\partial_{z^{\prime}} E_{y^{\prime}}\right)\right\}+ \\
+4 a_{1} b_{3}\left\{\left(\partial_{t} E_{x^{\prime}}\right)\left(\partial_{t} E_{z^{\prime}}\right)-c^{2}\left(\partial_{z^{\prime}} E_{x^{\prime}}\right)\left(\partial_{z^{\prime}} E_{z^{\prime}}\right)\right\} \\
\partial_{t}^{2} P_{y^{\prime}}+a_{2} b_{12} \partial_{t}^{2} P_{x^{\prime}}-a_{2} v_{g}^{2} \partial_{z^{\prime}}^{2} P_{y^{\prime}}= \\
=a_{2} b_{2}\left\{6\left[\left(\partial_{t} E_{y^{\prime}}\right)^{2}-c^{2}\left(\partial_{z^{\prime}} E_{y^{\prime}}\right)^{2}\right]+2\left[\left(\partial_{t} E_{x^{\prime}}\right)^{2}-c^{2}\left(\partial_{z^{\prime}} E_{x^{\prime}}\right)^{2}\right]+\right. \\
\left.+2\left[\left(\partial_{t} E_{z^{\prime}}\right)^{2}-c^{2}\left(\partial_{z^{\prime}} E_{z^{\prime}}\right)^{2}\right]\right\}+4 a_{2} b_{1}\left\{\left(\partial_{t} E_{x^{\prime}}\right)\left(\partial_{t} E_{y^{\prime}}\right)-c^{2}\left(\partial_{z^{\prime}} E_{x^{\prime}}\right)\left(\partial_{z^{\prime}} E_{y^{\prime}}\right)\right\}+ \\
+4 a_{2} b_{3}\left\{\left(\partial_{t} E_{z^{\prime}}\right)\left(\partial_{t} E_{y^{\prime}}\right)-c^{2}\left(\partial_{z^{\prime}} E_{z^{\prime}}\right)\left(\partial_{z^{\prime}} E_{y^{\prime}}\right)\right\}
\end{array}\right.
$$

where factors $a_{1}$ and $a_{2}$ were corrected with respect to (2.39), and a new coefficient $b_{3}$ was introduced:

$$
\begin{aligned}
& a_{1} \equiv a_{1}\left(E_{x^{\prime}}, E_{y^{\prime}}, E_{z^{\prime}}\right)=\left(1+4 \pi \chi^{(3)}\left(v_{g} / c\right)^{2}\left(3 E_{x^{\prime}}^{2}+E_{y^{\prime}}^{2}+E_{z^{\prime}}^{2}\right)\right)^{-1}, \\
& a_{2} \equiv a_{2}\left(E_{x^{\prime}}, E_{y^{\prime}}, E_{z^{\prime}}\right)=\left(1+4 \pi \chi^{(3)}\left(v_{g} / c\right)^{2}\left(E_{x^{\prime}}^{2}+3 E_{y^{\prime}}^{2}+E_{z^{\prime}}^{2}\right)\right)^{-1}, \\
& b_{3} \equiv b_{3}\left(E_{z^{\prime}}\right)=\chi^{(3)}\left(v_{g} / c\right)^{2} E_{z^{\prime}},
\end{aligned}
$$

while $b_{12}, b_{1}, b_{2}$ are defined in (2.39). Then the system (2.40) is coupled to the $3 \mathrm{~d}-2 \mathrm{~d}$ Maxwell-Schrödinger model.

Remark. For numerical implementation of the systems (2.38) and (2.40), it may be necessary to replace all the terms containing the time derivatives of the fields in the RHSs, just as it was done for transformation (2.35) to (2.36). 


\subsubsection{Global Model Under the Paraxial Approximation}

We consider the model that includes the wave equation for the electric field (2.22), the equation for the current of free electrons (2.28), and the polarization evolution equation (2.26). Recall that the paraxial approximation uses the envelope description of the pulse, which makes it admissible only for studying of long enough pulses. Thus, the approach below is not applicable for few-cycle pulses, and in this regard the model is less generic than the one represented in Section 2.3.2. However, the present model is more appropriate for studying the propagation of filament.

In principle, the interaction of strong laser field with the medium breaks symmetries of the incoming pulse, and in the strict sense, makes the paraxial approximation inapplicable. We derive a model when the paraxial approximation can still be assumed [23]. For simplicity, we limit the study to the case when only the fundamental and the third harmonics exist. Under the paraxial approximation, see (1.69), the electric field harmonics propagating in the direction $\mathbf{e}_{z^{\prime}}$ reads

$$
\mathbf{E}_{m}\left(\mathbf{x}_{\perp}^{\prime}, z^{\prime}, t\right)=A_{m}\left(\mathbf{x}_{\perp}^{\prime}, z^{\prime}, t\right) e^{\mathrm{i}\left(k_{m} z^{\prime}-m \omega t\right)} \mathbf{e}_{\perp}+\text { c.c. }, \quad m=1,3
$$

where "c.c." is used for the corresponding complex conjugate term, the symbol $\perp$ refers to the directions perpendicular to the wave propagation, $k_{m} \equiv k_{m z^{\prime}}$ denotes the $z^{\prime}$-component of the $m^{\text {th }}$ harmonics wave vector, and $A_{m}\left(\mathbf{x}_{\perp}^{\prime}, z^{\prime}, t\right)$ are the slow varying complex amplitudes. At time $t_{\beta}$ and at $\left(\mathbf{x}_{\perp, \alpha}^{\prime}, z_{\alpha}^{\prime}\right)$, the initial polarization $\mathbf{P}\left(\mathbf{x}_{\perp, \alpha}^{\prime}, t_{\beta}\right)$ and its derivative $\partial_{t} \mathbf{P}\left(\mathbf{x}_{\perp, \alpha}^{\prime}, t_{\beta}\right)$, are computed from a TDSE

$$
\mathrm{i} \partial_{t} \psi_{\alpha}=-\frac{1}{2} \triangle \psi_{\alpha}+V_{c}(\mathbf{x}) \psi_{\alpha}+\mathbf{x} \cdot \mathbf{E}(t) \psi_{\alpha}
$$

with initial data $\psi_{\alpha}\left(t_{\beta}\right)=\psi_{\alpha}\left(t_{\beta-1}\right)$, where $\psi_{\alpha}\left(t_{0}\right)$ corresponds to the ground state, according to (2.27). Next, from (2.26) we search for an evolution equation under the 
paraxial approximation for $\mathbf{P}_{m}(m=1,3)$ and $\mathbf{J}$ written in the form

$$
\begin{aligned}
\mathbf{P}_{m}\left(\mathbf{x}_{\perp}^{\prime}, z^{\prime}, t\right) & =\Pi_{m}\left(\mathbf{x}_{\perp}^{\prime}, z^{\prime}, t\right) e^{\mathrm{i}\left(k_{m}^{\prime} z^{\prime}-m \omega t\right)} \mathbf{e}_{\perp}+\text { c.c., } \quad m=1,3 \\
\mathbf{J}\left(\mathbf{x}_{\perp}^{\prime}, z^{\prime}, t\right) & =\Lambda\left(\mathbf{x}_{\perp}^{\prime}, z^{\prime}, t\right) e^{\mathbf{i}\left(k_{1} z^{\prime}-\omega t\right)} \mathbf{e}_{\perp}+\text { c.c. },
\end{aligned}
$$

where $\Pi_{m}, \Lambda$ are the slowly varying complex amplitudes. Note that we do account for a possible wavenumber mismatch between $\mathbf{E}_{m}$ and $\mathbf{P}_{m}$, implying that in general $k_{m} \neq k_{m}^{\prime}$. Thus, the system we are working on becomes

$$
\begin{aligned}
& \partial_{t}^{2} \mathbf{E}_{1}-c^{2} \triangle \mathbf{E}_{1}=-4 \pi \partial_{t}^{2} \mathbf{P}_{1}-4 \pi \partial_{t} \mathbf{J}, \\
& \partial_{t}^{2} \mathbf{E}_{3}-c^{2} \triangle \mathbf{E}_{3}=-4 \pi \partial_{t}^{2} \mathbf{P}_{3}, \\
& \partial_{t}^{2} \mathbf{P}_{1}-v_{g}^{2} \triangle \mathbf{P}_{1}=\left(\frac{v_{g}}{c}\right)^{2} \chi^{(3)}\left[\partial_{t}^{2}\left(\left|A_{1}\right|^{2} A_{1} e^{\mathrm{i}\left(k_{1} z^{\prime}-\omega t\right)}\right)-\right. \\
&\left.-c^{2} \triangle\left(\left|A_{1}\right|^{2} A_{1} e^{\mathrm{i}\left(k_{1} z^{\prime}-\omega t\right)}\right)+\text { c.c. }\right]-4 \pi\left(\frac{v_{g}}{c}\right)^{2} \chi^{(1)} \partial_{t} \mathbf{J}, \\
& \partial_{t}^{2} \mathbf{P}_{3}-v_{g}^{2} \triangle \mathbf{P}_{3}=\left(\frac{v_{g}}{c}\right)^{2} \chi^{(3)}\left[\partial_{t}^{2}\left(A_{1}^{3} e^{3 \mathbf{i}\left(k_{1} z^{\prime}-\omega t\right)}\right)-\right. \\
&\left.-c^{2} \triangle\left(A_{1}^{3} e^{3 \mathrm{i}\left(k_{1} z^{\prime}-\omega t\right)}\right)+\text { c.c. }\right], \\
& \partial_{t} \mathbf{J}+\nu_{e} \mathbf{E}_{1}= \mathcal{N}_{e} \mathbf{E}_{1} .
\end{aligned}
$$

From (2.43) the temporal derivative of the current of free electrons is

$$
\partial_{t} \mathbf{J}=\left(\partial_{t} \Lambda-i \omega \Lambda\right) e^{\mathrm{i}\left(k_{1} z^{\prime}-\omega t\right)} \mathbf{e}_{\perp},
$$

so that the equation (2.50) under the paraxial approximation reads

$$
\partial_{t} \Lambda=\left(i \omega-\nu_{e}\right) \Lambda+\mathcal{N}_{e} A_{1},
$$

resulting in a new expression for $\partial_{t} \mathbf{J}$, which we need to substitute in (2.44) and (2.46)

$$
\partial_{t} \mathbf{J}=\left(-\nu_{e} \Lambda+\mathcal{N}_{e} A_{1}\right) e^{i\left(k_{1} z^{\prime}-\omega t\right)} \mathbf{e}_{\perp}
$$


Now, we write the spatial and temporal derivatives of the field under the SVAE

$$
\left\{\begin{array}{l}
\triangle \mathbf{E}_{m}=\left(\triangle_{\perp} A_{m}+\partial_{z^{\prime}}^{2} A_{m}+2 \mathrm{i} k_{m} \partial_{z^{\prime}} A_{m}-k_{m}^{2} A_{m}\right) e^{\mathrm{i}\left(k_{m} z^{\prime}-m \omega t\right)} \mathbf{e}_{\perp}, \\
\partial_{t}^{2} \mathbf{E}_{m}=\left(\partial_{t}^{2} A_{m}-2 \mathrm{i} m \omega \partial_{t} A_{m}-m^{2} \omega^{2} A_{m}\right) e^{\mathrm{i}\left(k_{m} z^{\prime}-m \omega t\right)} \mathbf{e}_{\perp} .
\end{array}\right.
$$

The same expressions take place for $\mathbf{P}_{m}$ :

$$
\left\{\begin{array}{l}
\triangle \mathbf{P}_{m}=\left(\triangle_{\perp} \Pi_{m}+\partial_{z^{\prime}}^{2} \Pi_{m}+2 \mathrm{i} k_{m}^{\prime} \partial_{z^{\prime}} \Pi_{m}-\left(k_{m}^{\prime}\right)^{2} \Pi_{m}\right) e^{\mathrm{i}\left(k_{m}^{\prime} z^{\prime}-m \omega t\right)} \mathbf{e}_{\perp} \\
\partial_{t}^{2} \mathbf{P}_{m}=\left(\partial_{t}^{2} \Pi_{m}-2 \mathrm{i} m \omega \partial_{t} \Pi_{m}-m^{2} \omega^{2} \Pi_{m}\right) e^{\mathrm{i}\left(k_{m}^{\prime} z^{\prime}-m \omega t\right)} \mathbf{e}_{\perp},
\end{array}\right.
$$

and the nonlinear terms read:

$$
\left\{\begin{aligned}
\triangle\left(\left|A_{1}\right|^{2} A_{1} e^{\mathrm{i}\left(k_{1} z^{\prime}-\omega t\right)}\right)= & \left(\triangle_{\perp}\left(\left|A_{1}\right|^{2} A_{1}\right)+\partial_{z^{\prime}}^{2}\left(\left|A_{1}\right|^{2} A_{1}\right)+2 \mathrm{i} k_{1} \partial_{z^{\prime}}\left(\left|A_{1}\right|^{2} A_{1}\right)-\right. \\
& \left.-k_{1}^{2}\left|A_{1}\right|^{2} A_{1}\right) e^{\mathrm{i}\left(k_{1} z^{\prime}-\omega t\right)} \mathbf{e}_{\perp}, \\
\partial_{t}^{2}\left(\left|A_{1}\right|^{2} A_{1} e^{\mathrm{i}\left(k_{1} z^{\prime}-\omega t\right)}\right)= & \left(\partial_{t}^{2}\left(\left|A_{1}\right|^{2} A_{1}\right)-2 \mathrm{i} \omega \partial_{t}\left(\left|A_{1}\right|^{2} A_{1}\right)-\omega^{2}\left|A_{1}\right|^{2} A_{1}\right) e^{\mathrm{i}\left(k_{1} z^{\prime}-\omega t\right)} \mathbf{e}_{\perp},
\end{aligned}\right.
$$

and

$$
\left\{\begin{array}{l}
\triangle\left(A_{1}^{3} e^{3 \mathrm{i}\left(k_{1} z^{\prime}-\omega t\right)}\right)=\left(\triangle_{\perp} A_{1}^{3}+\partial_{z^{\prime}}^{2} A_{1}^{3}+6 \mathrm{i} k_{1} \partial_{z^{\prime}} A_{1}^{3}-9 k_{1}^{2} A_{1}^{3}\right) e^{3 \mathrm{i}\left(k_{1} z^{\prime}-\omega t\right)} \mathbf{e}_{\perp} \\
\partial_{t}^{2}\left(A_{1}^{3} e^{3 \mathrm{i}\left(k_{1} z^{\prime}-\omega t\right)}\right)=\left(\partial_{t}^{2} A_{1}^{3}-6 \mathrm{i} \omega \partial_{t} A_{1}^{3}-9 \omega^{2} A_{1}^{3}\right) e^{3 \mathrm{i}\left(k_{1} z^{\prime}-\omega t\right)} \mathbf{e}_{\perp}
\end{array}\right.
$$

To simplify all these expressions, we now apply the usual SVEA:

$$
\left\{\begin{array}{lll}
\left|\partial_{z^{\prime}}^{2} A_{n}\right| & \ll k_{z^{\prime}}\left|\partial_{z^{\prime}} A_{n}\right| & \ll k_{z^{\prime}}^{2}\left|A_{n}\right|, \\
\left|\partial_{z^{\prime}}^{2} \Pi_{n}\right| & \ll k_{z^{\prime}}\left|\partial_{z^{\prime}} \Pi_{n}\right| & \ll k_{z^{\prime}}^{2}\left|\Pi_{n}\right|, \\
\left.\left.\left|\partial_{z^{\prime}}^{2}\right| A_{1}\right|^{2} A_{1}\right) \mid & \ll k_{z^{\prime}}\left|\partial_{z^{\prime}}\left(\left|A_{1}\right|^{2} A_{1}\right)\right| & \ll k_{z^{\prime}}^{2}\left|A_{1}\right|^{3}, \\
\left|\partial_{z^{\prime}}^{2} A_{1}^{3}\right| & \ll k_{z^{\prime}}\left|\partial_{z^{\prime}} A_{1}^{3}\right| & \ll k_{z^{\prime}}^{2}\left|A_{1}^{3}\right|, \\
\left|\partial_{t}^{2} A_{n}\right| & \ll \omega\left|\partial_{t} A_{n}\right| & \ll \omega^{2}\left|A_{n}\right|, \\
\left|\partial_{t}^{2} \Pi_{n}\right| & \ll \omega\left|\partial_{t} \Pi_{n}\right| & \ll \omega^{2}\left|\Pi_{n}\right|, \\
\left|\partial_{t}^{2}\left(\left|A_{1}\right|^{2} A_{1}\right)\right| & \ll \omega\left|\partial_{t}\left(\left|A_{1}\right|^{2} A_{1}\right)\right| & \ll \omega^{2}\left|A_{1}\right|^{3}, \\
\left|\partial_{t}^{2} A_{1}^{3}\right| & \ll \omega\left|\partial_{t} A_{1}^{3}\right| & \ll \omega^{2}\left|A_{1}^{3}\right| .
\end{array}\right.
$$


Then, using the dispersion relations: $n(\omega) \omega=c k_{1}$ and $3 n(\omega) \omega=c k_{3}$ (however, here we assume for the refractive indexes $n(\omega)=n(3 \omega)=n_{0}=\sqrt{1+4 \pi \chi^{(1)}}$, see (2.18)), we get a complete model

$\triangleright$ MASP model coupled to the evolution equations under the paraxial approximation

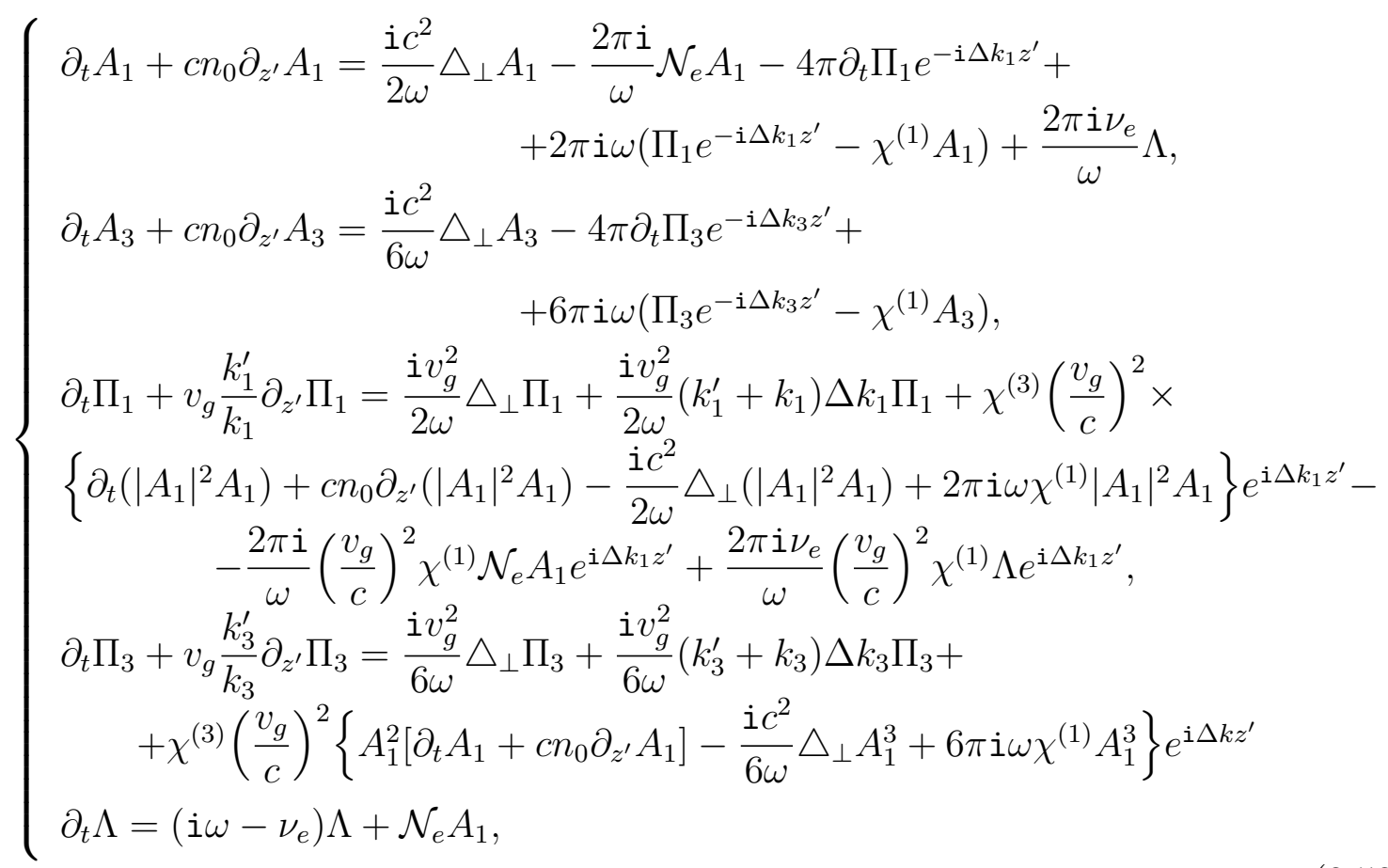

where $\Delta k_{1}=k_{1}-k_{1}^{\prime}, \Delta k_{3}=k_{3}-k_{3}^{\prime}$ and $\Delta k=3 k_{1}-k_{3}^{\prime}$.

To obtain from (2.52) a model, which is handier in implementation, we make several assumptions. First, since in the above approximation the refractive index $n_{0}$ does not depend on $\omega$, it follows that $k_{3}=3 k_{1}$ and $k_{3}^{\prime}=3 k_{1}^{\prime}$, hence $\Delta k=\Delta k_{3}=3 \Delta k_{1}$. Next, using the expansion for polarization up to the third order in $A_{1}$, see (1.50) and (1.51), we can simplify in the first and second equations: $\Pi_{1} e^{-\mathrm{i} \Delta k_{1} z^{\prime}}-\chi^{(1)} A_{1}=$ $\chi^{(3)} A_{1}\left|A_{1}\right|^{2}$ and $\Pi_{3} e^{-i \Delta k_{3} z^{\prime}}-\chi^{(1)} A_{3}=\chi^{(3)} A_{1}^{3}$. Finally, following the consideration of the Kerr effect, see (1.59), we assume that $\left|A_{1}\right|^{2}$ is constant in space and time, in other words, we use the constant-pump approximation: $\left|A_{3}\right| \ll\left|A_{1}\right|$. 
We discuss the possible computational realization of the model given by (2.52). As before, the pulse propagates along the $z^{\prime}$-direction, and the gas region is divided into $N_{1}$ subdomains containing $N_{2}$ Maxwell's cells each. At every time point, we compute the electric field (i.e. the envelopes $A_{1}$ and $A_{3}$ for the first and third harmonics), and the current of the free electrons, according to the next system obtained from (2.52):

$$
\left\{\begin{array}{l}
\partial_{t} A_{1}+c n_{0} \partial_{z^{\prime}} A_{1}-\frac{i c^{2}}{2 \omega} \triangle_{\perp} A_{1}-2 \pi i \omega \chi^{(3)} A_{1}\left|A_{1}\right|^{2}= \\
=-4 \pi \partial_{t} \Pi_{1} e^{-\frac{i}{3} \Delta k z^{\prime}}-\frac{2 \pi i}{\omega}\left(\partial_{t} \Lambda-i \omega \Lambda\right) \\
\partial_{t} A_{3}+c n_{0} \partial_{z^{\prime}} A_{3}-\frac{i c^{2}}{6 \omega} \triangle_{\perp} A_{3}-6 \pi i \omega \chi^{(3)} A_{1}^{3}=-4 \pi \partial_{t} \Pi_{3} e^{-i \Delta k z^{\prime}} \\
\partial_{t} \Lambda=\left(i \omega-\nu_{e}\right) \Lambda+\mathcal{N}_{e} A_{1}
\end{array}\right.
$$

As we can see from the first and second equations of the system, the LHS describes the transport of the envelope, its transversal diffusion and some nonlinearity effects, while the RHSs represent the responses of the bound and free (in the first equation) electrons. Finally, to close the model, we need three additional evolution equations: two for the complex envelopes of polarization $\Pi_{1}, \Pi_{3}$ and one for the electron density $\mathcal{N}_{e}$. Two first equations follow from (2.52), the third one can be naturally taken in the form of the transport equation:

$$
\left\{\begin{array}{l}
\partial_{t} \Pi_{1}+v_{g} a \frac{k_{1}^{\prime}}{k_{1}} \partial_{z^{\prime}} \Pi_{1}-\frac{i v_{g}^{2} a}{2 \omega} \triangle_{\perp} \Pi_{1}-\frac{i v_{g}^{2} a}{6 \omega}\left(k_{1}^{\prime}+k_{1}\right) \Delta k \Pi_{1}= \\
=\frac{2 \pi i a}{\omega}\left(\frac{v_{g}}{c}\right)^{2}\left(\nu_{e} \Lambda-\mathcal{N}_{e} A_{1}+\chi^{(3)} \omega^{2}\left|A_{1}\right|^{2} A_{1}\right)\left(\chi^{(1)}+\chi^{(3)}\left|A_{1}\right|^{2}\right) e^{i \Delta k z^{\prime} / 3} \\
\partial_{t} \Pi_{3}+v_{g} \frac{k_{3}^{\prime}}{k_{3}} \partial_{z^{\prime}} \Pi_{3}-\frac{i v_{g}^{2}}{6 \omega} \triangle_{\perp} \Pi_{3}-\frac{i v_{g}^{2}}{6 \omega}\left(k_{3}^{\prime}+k_{3}\right) \Delta k \Pi_{3}= \\
=-4 \pi\left(\frac{v_{g}}{c}\right)^{2} \chi^{(3)} e^{2 i \Delta k z^{\prime} / 3} A_{1}^{2} \partial_{t} \Pi_{1}-i \frac{\chi^{(3)}}{\omega}\left(\frac{v_{g}}{c}\right)^{2}\left\{c^{2} A_{1}\left(\nabla_{\perp} A_{1}\right)^{2}+\right. \\
\left.+2 \pi A_{1}^{2}\left(\mathcal{N}_{e} A_{1}-\nu_{e} \Lambda-3 \omega^{2} \chi^{(1)} A_{1}\right)\right\} e^{i \Delta k z^{\prime}} \\
\partial_{t} \mathcal{N}_{e}+v_{g} \partial_{z^{\prime}} \mathcal{N}_{e}=0
\end{array}\right.
$$

where $a=\left(1+4 \pi \chi^{(3)}\left(v_{g} / c\right)^{2}\left|A_{1}\right|^{2}\right)^{-1}$. Thus, at each time $t_{\beta}$, knowing $A_{1}, A_{3}$ and $\Lambda$ we can update the polarization using the first and the second equations of the system 
(2.54), with the initial conditions (2.27) computed from the set of TDSEs. Employing the last equation of the system with the initial condition (2.30), we can update the free electrons number density. Then at the next time step we proceed to compute the electric field from (2.53).

This model is attractive in the way that it provides an accurate description of the polarization envelope, and therefore allows for long pulse propagations, in particular, it can be applied for studying filament evolution. Naturally from (2.53) and (2.54) it is possible to derive even simpler models neglecting the difference between $k_{m}$ and $k_{m}^{\prime}$, so that $\Delta k=0$, and/or several nonlinear terms.

Finally we note that in Chapter 4 we present the examples of numerical implementation of several models derived in Section 2.3:

- The coupling of the simple evolution equation (2.17) to the $1 \mathrm{~d}-2 \mathrm{~d}$ and $3 \mathrm{~d}-1 \mathrm{~d}$ MASP models.

- The coupling of the general evolution equation (2.36) to the 1d-2d MASP model.

We left the remaining models discussed above for further investigations.

\subsection{Existence and Uniqueness of Weak Solutions for the MASP Models}

In this section, we are proving well-posedness of the some of the models derived in Sections 2.2 and 2.3. We start with the "pure" MASP model consisting of equations (2.1), (2.2) and (2.5). In this case, we base our proof on the results that were established earlier for the Schrödinger equation $[16,51,79]$ and the Maxwell-Schrödinger model [81]. Then, we discuss the existence and regularity of solutions to the model supplemented by the evolution equations for polarization (2.19), and for the free electrons density (2.29). 


\subsection{1 "Pure" MASP Model}

We denote by $\left(\mathbf{E}_{0}, \mathbf{B}_{0}, \mathbf{J}_{0}, \bar{\psi}_{0}\right)^{T}$ the initial data of the Equations (2.1), (2.2) and (2.5), where $\bar{\psi}_{0}=\left(\psi_{0,1} \ldots \psi_{0, m}\right)^{T}$, and $m$ is the total number of cells in the gas region. We suppose that $\mathbf{E}_{0}, \mathbf{B}_{0}, \mathbf{J}_{0}$ belong to $\left(H^{1}(\Omega)\right)^{3}$, where

- $\Omega \subset \mathbb{R}^{3}$ is a bounded spatial domain for Maxwell's equations (see Fig. 2.1) with a smooth boundary $\partial \Omega$ where Dirichlet zero BCs be posed for $\mathbf{E}, \mathbf{B}, \mathbf{J}$. In other words, we assume that the electromagnetic pulse remains always inside the domain $\Omega$, so that for all moments of time the flux of the Poynting vector $\mathbf{S}=\frac{c}{4 \pi} \mathbf{E} \times \mathbf{B}$ (recall, in our case $\mathbf{B} \equiv \mathbf{H}$ ) at the boundary $\partial \Omega$ is zero as well;

- $H^{1}(\Omega)$ denotes the Sobolev space $W^{k, p}(\Omega)$ of the order $\{k=1, p=2\}[115]$ :

$$
H^{1}(\Omega)=\left\{v \in L^{2}(\Omega) \mid \quad \partial_{x_{i}} v_{\mathcal{D}^{\prime}} \in L^{2}(\Omega), \quad i=1,2,3\right\}
$$

where the first derivative $\partial_{x_{i}} v_{\mathcal{D}^{\prime}}$ is defined in the distributional (weak) sense (i.e. $\mathcal{D}^{\prime}(\Omega)$ is a set of distributions on $\left.\Omega\right)$, while the Lebesgue spaces $L^{p}(1 \leq p<+\infty)$ are defined as:

$$
L^{p}(\Omega)=\left\{v:\left.\Omega \rightarrow \mathbb{R}\left|\|v\|_{L^{p}}^{p}=\int_{\Omega}\right| v(\mathbf{x})\right|^{p} d \mathbf{x}<+\infty\right\}
$$

Further in this section, we will omit the symbol $\mathcal{D}^{\prime}$ on the derivatives, for the sake of simplicity of the notation.

Recall that in the Born-Oppenheimer approximation the distance between nuclei $R_{0}$ is fixed, so that $R_{0} \in \mathbb{R}$ is constant positive parameter for the wavefunctions, supposing that $\bar{\psi}_{0} \in\left(H^{1}\left(\mathbb{R}^{3}\right) \cap H_{1}\left(\mathbb{R}^{3}\right)\right)^{m}$, where the norm of the Sobolev space $H^{1}\left(\mathbb{R}^{3}\right)$ reads

$$
\|\psi\|_{H^{1}}^{2}=\int_{\mathbb{R}^{3}}\left(\left|\psi\left(\mathbf{x}, R_{0}\right)\right|^{2}+\sum_{i=1}^{3}\left|\partial_{x_{i}} \psi\left(\mathbf{x}, R_{0}\right)\right|^{2}\right) d \mathbf{x} .
$$


The norm of another Sobolev space $H_{1}$ (with low index), which is the image of $H^{1}$ (with upper index) under the Fourier transform [16,115], is defined as:

$$
\|\psi\|_{H_{1}}^{2}=\int_{\mathbb{R}^{3}}\left(1+\left|\left(\mathbf{x}, \mathbf{R}_{0}\right)\right|^{2}\right)\left|\psi\left(\mathbf{x}, R_{0}\right)\right|^{2} d \mathbf{x}
$$

where $\left|\left(\mathbf{x}, \mathbf{R}_{0}\right)\right|^{2}:=\left|\mathbf{x}-\mathbf{R}_{0}\right|^{2}=\left|\mathbf{x}-\mathbf{e}_{0} R_{0}\right|^{2}$ with unit vector $\mathbf{e}_{0}$ that defines the orientation of the molecule.

Let us give three examples of acceptable and objectionable ICs for the wavefunction $\psi_{0}: \mathbb{R} \rightarrow \mathbb{C}$

1. First, we consider $\psi_{10}\left(x, R_{0}\right)=e^{-\left(x-R_{0} / 2\right)^{2}}+e^{-\left(x+R_{0} / 2\right)^{2}}$ that belongs to $H^{1}(\mathbb{R})$, since the integral (2.57) for $\left\|\psi_{10}\right\|_{H^{1}}^{2}$ is bounded. The integral (2.58) is convergent as well for $\psi_{10}\left(x, R_{0}\right)$, thereby we proved that wavefunction $\psi_{10} \in$ $H^{1}(\mathbb{R}) \cap H_{1}(\mathbb{R})$.

2. However, we cannot choose the IC as a combination of Heaviside step functions:

$$
\begin{aligned}
\psi_{20}\left(x, R_{0}\right)= & u\left(x+\frac{R_{0}}{2}+\alpha\right)-u\left(x+\frac{R_{0}}{2}-\alpha\right)+ \\
& +u\left(x-\frac{R_{0}}{2}+\alpha\right)-u\left(x-\frac{R_{0}}{2}-\alpha\right), \quad \alpha>0 .
\end{aligned}
$$

Although $\psi_{20}\left(x, R_{0}\right)$ belongs to $L^{2}(\mathbb{R})$ and $H_{1}(\mathbb{R})$, its derivative in distributional sense

$$
\begin{aligned}
\psi_{20}^{\prime}\left(x, R_{0}\right)= & \delta\left(x+\frac{R_{0}}{2}+\alpha\right)-\delta\left(x+\frac{R_{0}}{2}-\alpha\right)+ \\
& +\delta\left(x-\frac{R_{0}}{2}+\alpha\right)-\delta\left(x-\frac{R_{0}}{2}-\alpha\right)
\end{aligned}
$$

does not belong to $L^{2}(\mathbb{R})$, so that we conclude $\psi_{20}\left(x, R_{0}\right) \in H_{1}(\mathbb{R}) \backslash H^{1}(\mathbb{R})$.

3. Our last example is $\psi_{30}\left(x, R_{0}\right)=\frac{\sin \left(x+R_{0} / 2\right)}{x+R_{0} / 2}+\frac{\sin \left(x-R_{0} / 2\right)}{x-R_{0} / 2}$ that is definitely in $H^{1}(\mathbb{R})$, however $\left(x \pm R_{0} / 2\right) \psi_{30}\left(x, R_{0}\right)$ do not belong to $L^{2}(\mathbb{R})$ and we conclude $\psi_{30}\left(x, R_{0}\right) \in H^{1}(\mathbb{R}) \backslash H_{1}(\mathbb{R})$. 
In the following, we will respectively denote by $L^{2}, H^{1}, H_{1}$, i.e. without any arguments, the sets $L^{2}\left(\mathbb{R}^{3}\right), H^{1}\left(\mathbb{R}^{3}\right), H_{1}\left(\mathbb{R}^{3}\right)$. In addition, we will use a shorthand symbol for $L^{\infty}$ implying the common definition:

$$
L^{\infty}\left(\mathbb{R}^{3}\right)=\left\{\psi\left(\mathbb{R}^{3}\right)\left|\quad\|\psi\|_{L^{\infty}}=\operatorname{ess} \sup _{\mathbf{x} \in \mathbb{R}^{3}}\right| \psi(\mathbf{x}) \mid<+\infty\right\}
$$

Now we extend the Existence and Uniqueness Theorem [81], referred to solutions of the Maxwell-Shrödinger (MS) model, to the case of the Maxwell-Shrödinger-Plasma (MASP) model.

Theorem 2.1. Suppose that $\left(\mathbf{E}_{0}, \mathbf{B}_{0}, \mathbf{J}_{0}\right) \in\left(H^{1}(\Omega)\right)^{3} \times\left(H^{1}(\Omega)\right)^{3} \times\left(H^{1}(\Omega)\right)^{3}, \bar{\psi}_{0} \in$ $\left(H^{1} \cap H_{1}\right)^{m}, R_{0}>0$ is a constant for all $t \in \mathbb{R}_{+}$and $\mathcal{N} \in C_{0}^{\infty}(\Omega)$. Suppose that on the smooth boundary $\partial \Omega$ zero Dirichlet BCs are imposed for vectors $\mathbf{E}, \mathbf{B}, \mathbf{J}$ for all $t \in \mathbb{R}_{+}$. Then there exists a time $T>0$ and a unique solution $(\mathbf{E}, \mathbf{B}, \mathbf{J}, \bar{\psi}) \in$ $\left(L^{\infty}\left(0, T ;\left(H^{1}(\Omega)\right)^{3}\right) \cap H^{1}\left(0, T ;\left(L^{2}(\Omega)\right)^{3}\right)\right)^{3} \times L^{\infty}\left(0, T ;\left(H^{1} \cap H_{1}\right)^{m}\right)$ to Equations $(2.1)$, $(2.2),(2.5)$.

In order to proof this Theorem, we should prove several important intermediate results. The first lemma follows closely [81] and [16].

Lemma 2.1.1. Suppose that $\mathbf{E}\left(\mathbf{x}^{\prime}, \cdot\right) \in L^{\infty}(0, T)$ and $\partial_{t} \mathbf{E}\left(\mathbf{x}^{\prime}, \cdot\right) \in L^{1}(0, T)$ for $\mathbf{x}^{\prime}$ fixed in $\Omega$. We assume that $R_{0}>0$ is fixed for all $t \in \mathbb{R}_{+}$. Then for all $\psi_{0, i} \in H^{1} \cap H_{1}$, there exists $\psi_{i} \in L^{\infty}\left(0, T ; H^{1} \cap H_{1}\right)$ solution to the Schrödinger equation (2.2), and there exists a constant $C_{T}>0$ such that

$$
\left\|\psi_{i}\right\|_{L^{\infty}\left(0, T ; H^{1} \cap H_{1}\right)} \leq C_{T}\left\|\psi_{0, i}\right\|_{H^{1} \cap H_{1}}
$$

We provide the proof of this lemma in Appendix C. 
The next lemma from [81] generalizes the result of Lemma 2.1.1 on the vectorvalued function $\bar{\psi}=\left(\psi_{1} \ldots \psi_{m}\right)^{T}$ :

Lemma 2.1.2 (Lorin et al. [81]). Suppose given $\mathbf{E}\left(\mathbf{x}^{\prime}, \cdot\right) \in L^{\infty}(0, T)$ and $\partial_{t} \mathbf{E}\left(\mathbf{x}^{\prime}, \cdot\right) \in$ $L^{1}(0, T)$, for $\mathbf{x}^{\prime}$ fixed in $\Omega$. Then there exists $C_{T}>0$, such that for all $\bar{\psi}_{0} \in\left(H^{1} \cap H_{1}\right)^{m}$ there exists a solution $\bar{\psi} \in L^{\infty}\left(0, T ;\left(H^{1} \cap H_{1}\right)^{m}\right)$ and

$$
\|\bar{\psi}\|_{L^{\infty}\left(0, T ;\left(H^{1} \cap H_{1}\right)^{m}\right)} \leq C_{T}\left\|\bar{\psi}_{0}\right\|_{\left(H^{1} \cap H_{1}\right)^{m}} .
$$

Proof. It follows from the previous lemma.

Lemma 2.1.3 (Lorin et al. [81]). For all $T>0$, and $\bar{\psi} \in L^{\infty}\left(0, T ;\left(H^{1} \cap H_{1}\right)^{m}\right)$, $\mathbf{P} \in L^{\infty}\left(0, T ;\left(\mathcal{C}_{0}^{\infty}\right)^{3}\right)$.

Proof. Recall that $\chi_{\Omega_{i}}, \mathcal{N}$ belong to $\mathcal{C}_{0}^{\infty}(\Omega)$ and $\psi_{i} \in L^{\infty}\left(0, T ; H^{1} \cap H_{1}\right)$, for all $i=1, \ldots, m$, we then deduce that

$$
t \mapsto \int_{\mathbb{R}^{3} \times \mathbb{R}_{+}} \psi_{i}^{*}\left(R_{0}, \mathbf{x}, t\right) \mathbf{x} \psi_{i}\left(R_{0}, \mathbf{x}, t\right) \in L^{\infty}(0, T) .
$$

In particular for all $i=1, \ldots, m$, as $\psi_{i}$ belongs to $H^{1} \cap H_{1}$, and the integral is defined. Finally, by definition of

$$
\mathbf{P}=\mathcal{N}\left(\mathbf{x}^{\prime}\right) \sum_{i=1}^{m} \mathbf{P}_{i}=-\mathcal{N}\left(\mathbf{x}^{\prime}\right) \sum_{i=1}^{m} \chi_{\Omega_{i}}\left(\mathbf{x}^{\prime}\right) \int_{R^{3}} \psi_{i}^{*}\left(R_{0}, \mathbf{x}, t\right) \mathbf{x} \psi_{i}\left(R_{0}, \mathbf{x}, t\right) d \mathbf{x} d R_{0},
$$

which concludes the proof.

Lemma 2.1.4 (Lorin et al. [81]). For $\mathbf{x}^{\prime}$ fixed in $\Omega$ and $T>0, \partial_{t} \mathbf{P}\left(\mathbf{x}^{\prime}, \cdot\right) \in L^{\infty}(0, T)$ and $\partial_{t}\left(\nabla \cdot \mathbf{P}\left(\mathbf{x}^{\prime}, \cdot\right)\right) \in L^{\infty}(0, T)$. 
Proof. First, we rewrite that $\partial_{t} \mathbf{P}\left(\mathbf{x}^{\prime}, t\right)=\mathcal{N}\left(\mathbf{x}^{\prime}\right) \sum_{i=1}^{m} \partial_{t} \mathbf{P}_{i}\left(\mathbf{x}^{\prime}, t\right)$, where

$$
\begin{gathered}
\partial_{t} \mathbf{P}_{i}\left(\mathbf{x}^{\prime}, t\right)=\chi_{\Omega_{i}}\left(\mathbf{x}^{\prime}\right) \int_{R^{3}} \partial_{t} \psi_{i}^{*}\left(R_{0}, \mathbf{x}, t\right) \mathbf{x} \psi_{i}\left(R_{0}, \mathbf{x}, t\right) d \mathbf{x} d R_{0}+ \\
+\chi_{\Omega_{i}}\left(\mathbf{x}^{\prime}\right) \int_{R^{3}} \psi_{i}^{*}\left(R_{0}, \mathbf{x}, t\right) \mathbf{x} \partial_{t} \psi_{i}\left(R_{0}, \mathbf{x}, t\right) d \mathbf{x} d R_{0}
\end{gathered}
$$

As $\psi_{i} \in L^{\infty}\left(0, T ; H^{1} \cap H_{1}\right)$ and $\chi_{\Omega_{i}}, \mathcal{N} \in \mathcal{C}_{0}^{\infty}(\Omega)$ then $\partial_{t} \mathbf{P}_{i} \in L^{\infty}(0, T)$ for all $i=1, \ldots, m$, hence $\partial_{t} \mathbf{P} \in L^{\infty}(0, T)$. Also as, according to Lemma 2.1.3, $\nabla \cdot \mathbf{P}(\cdot, t) \in$ $\mathcal{C}_{0}^{\infty}(\Omega)$ at $t$ fixed, we also have that $\partial_{t}(\nabla \cdot \mathbf{P})\left(\mathbf{x}^{\prime}, \cdot\right) \in L^{\infty}(0, T)$.

Lemma 2.1.5. Suppose given $\mathcal{N}_{e 0} \in \mathcal{C}_{0}^{\infty}$, then for all $T>0$, and $\bar{\psi} \in L^{\infty}\left(0, T ;\left(H^{1} \cap\right.\right.$ $\left.\left.H_{1}\right)^{m}\right), \mathcal{N}_{e} \in L^{\infty}\left(0, T ; \mathcal{C}_{0}^{\infty}\right)$

Proof. We know that $\mathcal{N}$ and $\chi_{\Omega_{i}}$ being to $\mathcal{C}_{0}^{\infty}$. Following equations $(2.9),(2.10)$, the expression for density of free electron $\mathcal{N}_{e}\left(\mathbf{x}^{\prime}, t\right)$ at different $\mathbf{x}^{\prime}$ reads

$$
\mathcal{N}_{e}\left(\mathbf{x}^{\prime}, t\right)=\mathcal{N}_{e 0}\left(\mathbf{x}^{\prime}\right)+\mathcal{N}\left(\mathbf{x}^{\prime}\right) \sum_{i=1}^{m} \chi_{\Omega_{i}}\left(\mathbf{x}^{\prime}\right)\left(1-\int_{\Omega_{i}}\left|\psi_{i}\left(R_{0}, \mathbf{x}^{\prime}, t\right)\right|^{2} d \mathbf{x}\right) d R_{0}
$$

which proves the lemma.

Lemma 2.1.6. Suppose that $\left.\mathbf{E}(t)\right|_{\partial \Omega}=\left.\mathbf{B}(t)\right|_{\partial \Omega}=\mathbf{0} \in \mathbb{R}^{3}$, then for all $T>0$ and $\Omega \subset \mathbb{R}^{3}$

$$
\begin{aligned}
& \|\mathbf{E}(\cdot, T)\|_{\left(L^{2}(\Omega)\right)^{3}}^{2}+\|\mathbf{B}(\cdot, T)\|_{\left(L^{2}(\Omega)\right)^{3}}^{2}=\left\|\mathbf{E}_{0}(\cdot)\right\|_{\left(L^{2}(\Omega)\right)^{3}}^{2}+\left\|\mathbf{B}_{0}(\cdot)\right\|_{\left(L^{2}(\Omega)\right)^{3}}^{2} \\
& -8 \pi \int_{0}^{T} \int_{\Omega} \mathbf{E}\left(\mathbf{x}^{\prime}, t\right) \cdot \partial_{t} \mathbf{P}\left(\mathbf{x}^{\prime}, t\right) d \mathbf{x}^{\prime} d t-8 \pi \int_{0}^{T} \int_{\Omega} \mathbf{E}\left(\mathbf{x}^{\prime}, t\right) \cdot \mathbf{J}\left(\mathbf{x}^{\prime}, t\right) d \mathbf{x}^{\prime} d t,
\end{aligned}
$$

and

$$
\begin{gathered}
\|\nabla \cdot \mathbf{E}(\cdot, T)\|_{L^{2}(\Omega)}^{2}=\left\|\nabla \cdot \mathbf{E}_{0}(\cdot)\right\|_{L^{2}(\Omega)}^{2}-8 \pi \int_{0}^{T} \int_{\Omega} \nabla \cdot \mathbf{E}\left(\mathbf{x}^{\prime}, t\right) \partial_{t} \nabla \cdot \mathbf{P}\left(\mathbf{x}^{\prime}, t\right) d \mathbf{x}^{\prime} d t- \\
-8 \pi \int_{0}^{T} \int_{\Omega} \nabla \cdot \mathbf{E}\left(\mathbf{x}^{\prime}, t\right) \nabla \cdot \mathbf{J}\left(\mathbf{x}^{\prime}, t\right) d \mathbf{x}^{\prime} d t
\end{gathered}
$$


Proof. We respectively use Ampere-Maxwell's and Faraday-Maxwell's laws:

$$
\begin{aligned}
\partial_{t} \mathbf{E}\left(\mathbf{x}^{\prime}, t\right) & =c \nabla \times \mathbf{B}\left(\mathbf{x}^{\prime}, t\right)-4 \pi \partial_{t} \mathbf{P}\left(\mathbf{x}^{\prime}, t\right)-4 \pi \mathbf{J}\left(\mathbf{x}^{\prime}, t\right), \\
\partial_{t} \mathbf{B}\left(\mathbf{x}^{\prime}, t\right) & =-c \nabla \times \mathbf{E}\left(\mathbf{x}^{\prime}, t\right) .
\end{aligned}
$$

We take the scalar products of (2.62) with $\mathbf{E}$, and (2.63) with $\mathbf{B}$ that result in

$$
\begin{aligned}
\mathbf{E}\left(\mathbf{x}^{\prime}, t\right) \cdot \partial_{t} \mathbf{E}\left(\mathbf{x}^{\prime}, t\right)= & c \mathbf{E}\left(\mathbf{x}^{\prime}, t\right) \cdot\left[\nabla \times \mathbf{B}\left(\mathbf{x}^{\prime}, t\right)\right]- \\
& \quad-4 \pi \mathbf{E}\left(\mathbf{x}^{\prime}, t\right) \cdot \partial_{t} \mathbf{P}\left(\mathbf{x}^{\prime}, t\right)-4 \pi \mathbf{E}\left(\mathbf{x}^{\prime}, t\right) \cdot \mathbf{J}\left(\mathbf{x}^{\prime}, t\right), \\
\mathbf{B}\left(\mathbf{x}^{\prime}, t\right) \cdot \partial_{t} \mathbf{B}\left(\mathbf{x}^{\prime}, t\right)= & -c \mathbf{B}\left(\mathbf{x}^{\prime}, t\right) \cdot\left[\nabla \times \mathbf{E}\left(\mathbf{x}^{\prime}, t\right)\right] .
\end{aligned}
$$

Noting that in LHS $\mathbf{E}\left(\mathbf{x}^{\prime}, t\right) \cdot \partial_{t} \mathbf{E}\left(\mathbf{x}^{\prime}, t\right)=\frac{1}{2} \partial_{t} \mathbf{E}^{2}\left(\mathbf{x}^{\prime}, t\right)$ and $\mathbf{B}\left(\mathbf{x}^{\prime}, t\right) \cdot \partial_{t} \mathbf{B}\left(\mathbf{x}^{\prime}, t\right)=$ $\frac{1}{2} \partial_{t} \mathbf{B}^{2}\left(\mathbf{x}^{\prime}, t\right)$, so that we obtain after integration over $(0, T) \times \Omega$ and by definition of $L^{2}$-norm (2.56):

$$
\begin{aligned}
\int_{0}^{T} \int_{\Omega} \mathbf{E}\left(\mathbf{x}^{\prime}, t\right) \cdot \partial_{t} \mathbf{E}\left(\mathbf{x}^{\prime}, t\right) d \mathbf{x}^{\prime} d t & =\frac{1}{2}\left(\|\mathbf{E}(\cdot, T)\|_{\left(L^{2}(\Omega)\right)^{3}}^{2}-\left\|\mathbf{E}_{0}(\cdot)\right\|_{\left(L^{2}(\Omega)\right)^{3}}^{2},\right. \\
\int_{0}^{T} \int_{\Omega} \mathbf{B}\left(\mathbf{x}^{\prime}, t\right) \cdot \partial_{t} \mathbf{B}\left(\mathbf{x}^{\prime}, t\right) d \mathbf{x}^{\prime} d t & =\frac{1}{2}\left(\|\mathbf{B}(\cdot, T)\|_{\left(L^{2}(\Omega)\right)^{3}}^{2}-\left\|\mathbf{B}_{0}(\cdot)\right\|_{\left(L^{2}(\Omega)\right)^{3}}^{2} .\right.
\end{aligned}
$$

We add the expressions (2.64) using:

$$
\nabla \cdot[\mathbf{E} \times \mathbf{B}]=\mathbf{B} \cdot[\nabla \times \mathbf{E}]-\mathbf{E} \cdot[\nabla \times \mathbf{B}]
$$

and integrating over $(0, T) \times \Omega$. Finally, using the divergence theorem, we deduce $(2.60)$.

Now we apply the operator $\nabla$ to the both sides of (2.62). Since the divergence of the curl is 0 , we obtain the equation

$$
\partial_{t} \nabla \cdot \mathbf{E}\left(\mathbf{x}^{\prime}, t\right)=-4 \pi \partial_{t} \nabla \cdot \mathbf{P}\left(\mathbf{x}^{\prime}, t\right)-4 \pi \nabla \cdot \mathbf{J}\left(\mathbf{x}^{\prime}, t\right)
$$


We take the product of the scalar equation (2.65) with a scalar $\nabla \cdot \mathbf{E}$ and again integrate over $(0, T) \times \Omega$, with Definition (2.56), resulting in (2.61).

Remark 1. Note that (2.60) corresponds to an energy conservation law in the bounded region $\Omega$. Indeed, introducing the displacement vector $\mathbf{D}=\mathbf{E}+4 \pi \mathbf{P}$ and recalling the expression for time derivative of the EM energy density in a dielectric medium (with $\mathbf{H} \equiv \mathbf{B}$ ) [70]:

$$
\partial_{t} W=\frac{1}{4 \pi} \int_{\Omega}\left(\mathbf{E}\left(\mathbf{x}^{\prime}, t\right) \cdot \partial_{t} \mathbf{D}\left(\mathbf{x}^{\prime}, t\right)+\mathbf{B}\left(\mathbf{x}^{\prime}, t\right) \cdot \partial_{t} \mathbf{B}\left(\mathbf{x}^{\prime}, t\right)\right) d \mathbf{x}^{\prime},
$$

we obtain by integrating the sum of Equations (2.64) over $\Omega$ the result known as Poynting's theorem [70]:

$$
\partial_{t} W=-\int_{\partial \Omega} \mathbf{S}\left(\mathbf{x}_{\perp}^{\prime}, t\right) \cdot d \mathbf{x}_{\perp}^{\prime}-\int_{\Omega} \mathbf{E}\left(\mathbf{x}^{\prime}, t\right) \cdot \mathbf{J}\left(\mathbf{x}^{\prime}, t\right) d \mathbf{x}^{\prime},
$$

where $\mathbf{S}\left(\mathbf{x}_{\perp}^{\prime}, t\right)$ represents the flux of electromagnetic energy (Poynting's vector) and $d \mathbf{x}_{\perp}^{\prime}$ is an element of surface $\partial \Omega$ oriented outside of $\Omega$. Thus, in its integral form (2.66) Poynting's theorem states that the decreasing of the EM energy in the volume is equal to the energy flux across its boundary and the work of the electric field on moving of free charges. Note that due to the BCs on $\mathbf{E}$ and $\mathbf{B}$, it follows that $\mathbf{S}\left(\mathbf{x}^{\prime}{ }_{\perp}, t\right) \equiv 0$, so that $\partial_{t} W=-\int_{\Omega} \mathbf{E}\left(\mathbf{x}^{\prime}, t\right) \cdot \mathbf{J}\left(\mathbf{x}^{\prime}, t\right) d \mathbf{x}^{\prime}$.

In turn, (2.65) expresses the conservation of the local free electron density $\mathcal{N}_{e}\left(\mathbf{x}^{\prime}, t\right)$ :

$$
\partial_{t} \mathcal{N}_{e}\left(\mathbf{x}^{\prime}, t\right)+\nabla \cdot \mathbf{J}\left(\mathbf{x}^{\prime}, t\right)=0
$$

as $\nabla \cdot \mathbf{E}\left(\mathbf{x}^{\prime}, t\right)+4 \pi \nabla \cdot \mathbf{P}\left(\mathbf{x}^{\prime}, t\right)=\nabla \cdot \mathbf{D}\left(\mathbf{x}^{\prime}, t\right)=4 \pi \mathcal{N}_{e}\left(\mathbf{x}^{\prime}, t\right)$. 
Lemma 2.1.7. Suppose that $\left.\mathbf{E}(t)\right|_{\partial \Omega}=\left.\mathbf{B}(t)\right|_{\partial \Omega}=\mathbf{0} \in \mathbb{R}^{3}$, then for all $T>0$ and $\Omega \subset \mathbb{R}^{3}$

$$
\begin{gathered}
\left\|\partial_{t} \mathbf{E}(\cdot, T)\right\|_{\left(L^{2}(\Omega)\right)^{3}}^{2}+c^{2}\|\nabla \times \mathbf{E}(\cdot, T)\|_{\left(L^{2}(\Omega)\right)^{3}}^{2}=\left\|\partial_{t} \mathbf{E}_{0}(\cdot)\right\|_{\left(L^{2}(\Omega)\right)^{3}}^{2}+ \\
+\left\|\nabla \times \mathbf{E}_{0}(\cdot)\right\|^{2}\left(L^{2}(\Omega)\right)^{3}-8 \pi \int_{0}^{T} \int_{\Omega} \partial_{t} \mathbf{E}\left(\mathbf{x}^{\prime}, t\right) \cdot \partial_{t}^{2} \mathbf{P}\left(\mathbf{x}^{\prime}, t\right) d \mathbf{x}^{\prime} d t- \\
\quad-8 \pi \int_{0}^{T} \int_{\Omega} \partial_{t} \mathbf{E}\left(\mathbf{x}^{\prime}, t\right) \cdot \partial_{t} \mathbf{J}\left(\mathbf{x}^{\prime}, t\right) d \mathbf{x}^{\prime} d t
\end{gathered}
$$

and

$$
\begin{gathered}
\left\|\partial_{t} \mathbf{B}(\cdot, T)\right\|_{\left(L^{2}(\Omega)\right)^{3}}^{2}+c^{2}\|\nabla \times \mathbf{B}(\cdot, T)\|_{\left(L^{2}(\Omega)\right)^{3}}^{2}=\left\|\partial_{t} \mathbf{B}_{0}(\cdot)\right\|_{\left(L^{2}(\Omega)\right)^{3}}^{2} \\
+\left\|\nabla \times \mathbf{B}_{0}(\cdot)\right\|^{2}\left(L^{2}(\Omega)\right)^{3}+8 \pi c \int_{0}^{T} \int_{\Omega} \partial_{t} \mathbf{B}\left(\mathbf{x}^{\prime}, t\right) \cdot \partial_{t}\left[\nabla \times \mathbf{P}\left(\mathbf{x}^{\prime}, t\right)\right] d \mathbf{x}^{\prime} d t+ \\
+8 \pi c \int_{0}^{T} \int_{\Omega} \partial_{t} \mathbf{B}\left(\mathbf{x}^{\prime}, t\right) \cdot\left[\nabla \times \mathbf{J}\left(\mathbf{x}^{\prime}, t\right)\right] d \mathbf{x}^{\prime} d t
\end{gathered}
$$

Proof. We differentiate (2.62) in time and then multiply both sides by $\partial_{t} \mathbf{E}$. We note that

$$
\begin{gathered}
\partial_{t} \mathbf{E} \cdot \partial_{t}^{2} \mathbf{E}=\frac{1}{2} \partial_{t}\left(\partial_{t} \mathbf{E} \cdot \partial_{t} \mathbf{E}\right)=\frac{1}{2} \partial_{t}\left|\partial_{t} \mathbf{E}\right|^{2} \\
\partial_{t} \mathbf{E} \cdot[\nabla \times[\nabla \times \mathbf{E}]]=\nabla \cdot\left[[\nabla \times \mathbf{E}] \times \partial_{t} \mathbf{E}\right]+[\nabla \times \mathbf{E}] \cdot\left[\nabla \times \partial_{t} \mathbf{E}\right]= \\
=\nabla \cdot\left[[\nabla \times \mathbf{E}] \times \partial_{t} \mathbf{E}\right]+\frac{1}{2} \partial_{t}|\nabla \times \mathbf{E}|^{2}
\end{gathered}
$$

We integrate the equation over $(0, T) \times \Omega$, apply the divergence theorem and obtain (2.67). Similarly, by differentiation (2.63) in time, then by multiplication by $\partial_{t} \mathbf{B}$ and finally by integration over $(0, T) \times \Omega$ we come to $(2.68)$.

Lemma 2.1.8. For all $T>0$,

$$
\begin{gathered}
\|\mathbf{J}(\cdot, T)\|_{\left(L^{2}(\Omega)\right)^{3}}^{2}=\left\|\mathbf{J}_{0}(\cdot)\right\|_{\left(L^{2}(\Omega)\right)^{3}}^{2}-2 \nu_{e} \int_{0}^{T}\|\mathbf{J}(\cdot, t)\|_{\left(L^{2}(\Omega)\right)^{3}}^{2} d t+ \\
+2 \int_{0}^{T} \int_{\Omega} \mathcal{N}_{e}\left(\mathbf{x}^{\prime}, t\right) \mathbf{J}\left(\mathbf{x}^{\prime}, t\right) \cdot \mathbf{E}\left(\mathbf{x}^{\prime}, t\right) d \mathbf{x}^{\prime} d t
\end{gathered}
$$


and

$$
\begin{gathered}
\left\|\partial_{t} \mathbf{J}(\cdot, T)\right\|_{\left(L^{2}(\Omega)\right)^{3}}^{2}=\left\|\partial_{t} \mathbf{J}_{0}(\cdot)\right\|_{\left(L^{2}(\Omega)\right)^{3}}^{2}-2 \nu_{e} \int_{0}^{T}\left\|\partial_{t} \mathbf{J}(\cdot, t)\right\|_{\left(L^{2}(\Omega)\right)^{3}}^{2} d t+ \\
+2 \int_{0}^{T} \int_{\Omega} \mathcal{N}_{e}\left(\mathbf{x}^{\prime}, t\right) \partial_{t} \mathbf{J}\left(\mathbf{x}^{\prime}, t\right) \cdot \partial_{t} \mathbf{E}\left(\mathbf{x}^{\prime}, t\right) d \mathbf{x}^{\prime} d t+ \\
+2 \int_{0}^{T} \int_{\Omega} \partial_{t} \mathcal{N}_{e}\left(\mathbf{x}^{\prime}, t\right) \partial_{t} \mathbf{J}\left(\mathbf{x}^{\prime}, t\right) \cdot \mathbf{E}\left(\mathbf{x}^{\prime}, t\right) d \mathbf{x}^{\prime} d t
\end{gathered}
$$

and

$$
\begin{gathered}
\|\nabla \cdot \mathbf{J}(\cdot, T)\|_{L^{2}(\Omega)}^{2}=\left\|\nabla \cdot \mathbf{J}_{0}(\cdot)\right\|_{L^{2}(\Omega)}^{2}-2 \nu_{e} \int_{0}^{T}\|(\nabla \cdot \mathbf{J})(\cdot, t)\|_{L^{2}(\Omega)}^{2} d t+ \\
+2 \int_{0}^{T} \int_{\Omega} \nabla \mathcal{N}_{e}\left(\mathbf{x}^{\prime}, t\right) \cdot \mathbf{E}\left(\mathbf{x}^{\prime}, t\right) \nabla \cdot \mathbf{J}\left(\mathbf{x}^{\prime}, t\right) d \mathbf{x}^{\prime} d t+ \\
\quad+2 \int_{0}^{T} \int_{\Omega} \mathcal{N}_{e}\left(\mathbf{x}^{\prime}, t\right) \nabla \cdot \mathbf{E}\left(\mathbf{x}^{\prime}, t\right) \nabla \cdot \mathbf{J}\left(\mathbf{x}^{\prime}, t\right) d \mathbf{x}^{\prime} d t
\end{gathered}
$$

and

$$
\begin{gathered}
\|\nabla \times \mathbf{J}(\cdot, T)\|_{\left(L^{2}(\Omega)\right)^{3}}^{2}=\left\|\nabla \times \mathbf{J}_{0}(\cdot)\right\|_{\left(L^{2}(\Omega)\right)^{3}}^{2}-2 \nu_{e} \int_{0}^{T}\|(\nabla \times \mathbf{J})(\cdot, t)\|_{\left(L^{2}(\Omega)\right)^{3}}^{2} d t+ \\
+2 \int_{0}^{T} \int_{\Omega}\left[\nabla \mathcal{N}_{e}\left(\mathbf{x}^{\prime}, t\right) \times \mathbf{E}\left(\mathbf{x}^{\prime}, t\right)\right] \cdot\left[\nabla \times \mathbf{J}\left(\mathbf{x}^{\prime}, t\right)\right] d \mathbf{x}^{\prime} d t+ \\
+2 \int_{0}^{T} \int_{\Omega} \mathcal{N}_{e}\left(\mathbf{x}^{\prime}, t\right)\left[\nabla \times \mathbf{E}\left(\mathbf{x}^{\prime}, t\right)\right] \cdot\left[\nabla \times \mathbf{J}\left(\mathbf{x}^{\prime}, t\right)\right] d \mathbf{x}^{\prime} d t .
\end{gathered}
$$

Proof. We take the scalar product of (2.5) with $\mathbf{J}$

$$
\frac{1}{2} \partial_{t}\left|\mathbf{J}\left(\mathbf{x}^{\prime}, t\right)\right|^{2}=-\nu_{e}\left|\mathbf{J}\left(\mathbf{x}^{\prime}, t\right)\right|^{2}+\mathcal{N}_{e}\left(\mathbf{x}^{\prime}, t\right) \mathbf{J}\left(\mathbf{x}^{\prime}, t\right) \cdot \mathbf{E}\left(\mathbf{x}^{\prime}, t\right)
$$

Integrating over $(0, T)$, gives (2.69). To prove (2.70), we differentiate equation $(2.5)$ in time, then we take the scalar product of both parts with $\partial_{t} \mathbf{J}$ and integrate over $(0, T)$.

By applying $\nabla \cdot$ on $(2.5)$, and then integrating over $(0, T)$ with $\nabla \cdot \mathbf{J}$, we deduce (2.71). Finally, by applying $\nabla \times$ on $(2.5)$, and then integrating over $(0, T)$ with $\nabla \times \mathbf{J}$, we deduce (2.72) 
Remark 2 . With a view of proper management of the physical variable dimensions in the following lemmas, we should make a remark. Recall that we use atomic units for all the equations, so that from MEs (2.1) we can conclude that $\operatorname{dim}(\mathbf{E})=\mathrm{T} \operatorname{dim}(\mathbf{J})$, where $\mathrm{T}$ is the symbol for the time dimension. Taking into account Drude's model (2.5), we can see that $\operatorname{dim}\left(\mathcal{N}_{e}\right)=\mathrm{T}^{-2}$. In the following, we introduce a constant $\eta>0$, having the dimension of time, $\operatorname{dim}(\eta)=\mathrm{T}$.

Lemma 2.1.9. There exists a constant $C>0$ such that for all time $T>0$

$$
\sup _{0 \leq t \leq T}\|\mathbf{E}(\cdot, t)\|_{\left(H^{1}(\Omega)\right)^{3}}^{2}+\sup _{0 \leq t \leq T}\|\mathbf{B}(\cdot, t)\|_{\left(H^{1}(\Omega)\right)^{3}}^{2}+\eta^{2} \sup _{0 \leq t \leq T}\|\mathbf{J}(\cdot, t)\|_{\left(H^{1}(\Omega)\right)^{3}}^{2} \leq C .
$$

Proof. From the result (2.60) of Lemma 2.1.6 we have for all $t \in(0, T]$

$$
\begin{aligned}
& \|\mathbf{E}(\cdot, T)\|_{\left(L^{2}(\Omega)\right)^{3}}^{2}+\|\mathbf{B}(\cdot, T)\|_{\left(L^{2}(\Omega)\right)^{3}}^{2} \leq\left\|\mathbf{E}_{0}(\cdot)\right\|_{\left(L^{2}(\Omega)\right)^{3}}^{2}+\left\|\mathbf{B}_{0}(\cdot)\right\|_{\left(L^{2}(\Omega)\right)^{3}}^{2}+ \\
& +8 \pi \int_{0}^{T} \int_{\Omega}\left|\mathbf{E}\left(\mathbf{x}^{\prime}, t\right) \cdot \partial_{t} \mathbf{P}\left(\mathbf{x}^{\prime}, t\right)\right| d \mathbf{x}^{\prime} d t+8 \pi \int_{0}^{T} \int_{\Omega^{2}}\left|\mathbf{E}\left(\mathbf{x}^{\prime}, t\right) \cdot \mathbf{J}\left(\mathbf{x}^{\prime}, t\right)\right| d \mathbf{x}^{\prime} d t \leq \\
& \leq\left\|\mathbf{E}_{0}(\cdot)\right\|_{\left(L^{2}(\Omega)\right)^{3}}^{2}+\left\|\mathbf{B}_{0}(\cdot)\right\|_{\left(L^{2}(\Omega)\right)^{3}}^{2}+8 \pi \eta^{-1} \int_{0}^{T^{2}}\|\mathbf{E}(\cdot, t)\|_{\left(L^{2}(\Omega)\right)^{3}}^{2} d t+ \\
& 4 \pi \eta \int_{0}^{T}\left\|\partial_{t} \mathbf{P}(\cdot, t)\right\|_{\left(L^{2}(\Omega)\right)^{3}}^{2} d t++4 \pi \eta \int_{0}^{T}\|\mathbf{J}(\cdot, t)\|_{\left(L^{2}(\Omega)\right)^{3}}^{{ }^{3}} d t .
\end{aligned}
$$

Similarly from (2.61):

$$
\begin{gathered}
\|\nabla \cdot \mathbf{E}(\cdot, T)\|_{L^{2}(\Omega)}^{2} \leq\left\|\nabla \cdot \mathbf{E}_{0}(\cdot)\right\|_{L^{2}(\Omega)}^{2}+8 \pi \int_{0}^{T} \int_{\Omega}\left|\nabla \cdot \mathbf{E}\left(\mathbf{x}^{\prime}, t\right) \partial_{t} \nabla \cdot \mathbf{P}\left(\mathbf{x}^{\prime}, t\right)\right| d \mathbf{x}^{\prime} d t+ \\
+8 \pi \int_{0}^{T} \int_{\Omega}\left|\nabla \cdot \mathbf{E}\left(\mathbf{x}^{\prime}, t\right) \nabla \cdot \mathbf{J}\left(\mathbf{x}^{\prime}, t\right)\right| d \mathbf{x}^{\prime} d t \leq\left\|\nabla \cdot \mathbf{E}_{0}(\cdot)\right\|_{L^{2}(\Omega)}^{2}+ \\
+8 \pi \eta^{-1} \int_{0}^{T}\|\nabla \cdot \mathbf{E}(\cdot, t)\|_{L^{2}(\Omega)}^{2} d t+4 \pi \eta \int_{0}^{T}\left\|\partial_{t} \nabla \cdot \mathbf{P}(\cdot, t)\right\|_{L^{2}(\Omega)}^{2} d t+ \\
+4 \pi \eta \int_{0}^{T}\|\nabla \cdot \mathbf{J}(\cdot, t)\|_{L^{2}(\Omega)}^{2} d t .
\end{gathered}
$$


From Lemma 2.1.5 and (2.69) of Lemma 2.1.8, we have for all $t \in(0, T]$

$$
\begin{aligned}
& \|\mathbf{J}(\cdot, T)\|_{\left(L^{2}(\Omega)\right)^{3}}^{2} \leq\left\|\mathbf{J}_{0}(\cdot)\right\|_{\left(L^{2}(\Omega)\right)^{3}}^{2}+2 \nu_{e} \int_{0}^{T}\|\mathbf{J}(\cdot, t)\|_{\left(L^{2}(\Omega)\right)^{3}}^{2} d t+ \\
& \quad+2 \int_{0}^{T} \int_{\Omega} \mathcal{N}_{e}\left(\mathbf{x}^{\prime}, t\right)\left|\mathbf{J}\left(\mathbf{x}^{\prime}, t\right) \cdot \mathbf{E}\left(\mathbf{x}^{\prime}, t\right)\right| d \mathbf{x}^{\prime} d t \leq\left\|\mathbf{J}_{0}(\cdot)\right\|^{2}\left(L^{2}(\Omega)\right)^{3}+ \\
& +\left(2 \nu_{e}+\eta^{-1}\right) \int_{0}^{T}\|\mathbf{J}(\cdot, t)\|_{\left(L^{2}(\Omega)\right)^{3}}^{2} d t+\eta \sup _{\Omega,[0, T]} \mathcal{N}_{e}^{2}\left(\mathbf{x}^{\prime}, t\right) \int_{0}^{T}\|\mathbf{E}(\cdot, t)\|_{\left(L^{2}(\Omega)\right)^{3}}^{{ }^{3}} d t,
\end{aligned}
$$

and with Lemma 2.1.5 and (2.71)

$$
\begin{gathered}
\|\nabla \cdot \mathbf{J}(\cdot, T)\|_{L^{2}(\Omega)}^{2} \leq\left\|\nabla \cdot \mathbf{J}_{0}(\cdot)\right\|_{L^{2}(\Omega)}^{2}+2 \nu_{e} \int_{0}^{T} \int_{\Omega}\left|(\nabla \cdot \mathbf{J})\left(\mathbf{x}^{\prime}, t\right)\right|^{2} d \mathbf{x}^{\prime} d t+ \\
+2 \int_{0}^{T} \int_{\Omega}\left|\nabla \mathcal{N}_{e}\left(\mathbf{x}^{\prime}, t\right) \cdot \mathbf{E}\left(\mathbf{x}^{\prime}, t\right) \nabla \cdot \mathbf{J}\left(\mathbf{x}^{\prime}, t\right)\right| d \mathbf{x}^{\prime} d t+ \\
+2 \int_{0}^{T} \int_{\Omega}\left|\mathcal{N}_{e}\left(\mathbf{x}^{\prime}, t\right) \nabla \cdot \mathbf{E}\left(\mathbf{x}^{\prime}, t\right) \nabla \cdot \mathbf{J}\left(\mathbf{x}^{\prime}, t\right)\right| d \mathbf{x}^{\prime} d t \leq\left\|\nabla \cdot \mathbf{J}_{0}(\cdot)\right\|_{L^{2}(\Omega)}^{2}+ \\
+2\left(\nu_{e}+\eta^{-1}\right) \int_{0}^{T}\|(\nabla \cdot \mathbf{J})(\cdot, t)\|_{L^{2}(\Omega)}^{2} d t+ \\
+\eta \sup _{\Omega,[0, T]}\left(\nabla \mathcal{N}_{e}\left(\mathbf{x}^{\prime}, t\right)\right)^{2} \int_{0}^{T}\|\mathbf{E}(\cdot, t)\|_{L^{2}(\Omega)}^{2} d t+ \\
\quad+\eta \sup _{\Omega,[0, T]} \mathcal{N}_{e}^{2}\left(\mathbf{x}^{\prime}, t\right) \int_{0}^{T}\|\nabla \cdot \mathbf{E}(\cdot, t)\|_{L^{2}(\Omega)}^{2} d \mathbf{x}^{\prime} d t .
\end{gathered}
$$

Combining the first and third inequalities, we get:

$$
\begin{aligned}
& \|\mathbf{E}(\cdot, T)\|_{\left(L^{2}(\Omega)\right)^{3}}^{2}+\|\mathbf{B}(\cdot, T)\|_{\left(L^{2}(\Omega)\right)^{3}}^{2}+\eta^{2}\|\mathbf{J}(\cdot, T)\|_{\left(L^{2}(\Omega)\right)^{3}}^{2} \leq \\
& \leq\left\|\mathbf{E}_{0}(\cdot)\right\|_{\left(L^{2}(\Omega)\right)^{3}}^{2}+\left\|\mathbf{B}_{0}(\cdot)\right\|_{\left(L^{2}(\Omega)\right)^{3}}^{2}+\eta^{2}\left\|\mathbf{J}_{0}(\cdot)\right\|_{\left(L^{2}(\Omega)\right)^{3}}^{2}+ \\
& \quad+\left(8 \pi \eta^{-1}+\eta^{3} \sup _{\Omega,[0, T]} \mathcal{N}_{e}^{2}\left(\mathbf{x}^{\prime}, t\right)\right) \int_{0}^{T}\|\mathbf{E}(\cdot, t)\|_{\left(L^{2}(\Omega)\right)^{3}}^{2} d t+ \\
& +\left(4 \pi \eta+2 \nu_{e} \eta^{2}+\eta\right) \int_{0}^{T}\|\mathbf{J}(\cdot, t)\|_{\left(L^{2}(\Omega)\right)^{3}}^{2} d t+4 \pi \eta \int_{0}^{T}\left\|\partial_{t} \mathbf{P}(\cdot, t)\right\|_{\left(L^{2}(\Omega)\right)^{3}}^{2} d t .
\end{aligned}
$$


Identically for the second and forth inequalities:

$$
\begin{gathered}
\|\nabla \cdot \mathbf{E}(\cdot, T)\|_{L^{2}(\Omega)}^{2}+\eta^{2}\|\nabla \cdot \mathbf{J}(\cdot, T)\|_{L^{2}(\Omega)}^{2} \leq\left\|\nabla \cdot \mathbf{E}_{0}(\cdot)\right\|_{L^{2}(\Omega)}^{2}+\eta^{2}\left\|\nabla \cdot \mathbf{J}_{0}(\cdot)\right\|_{L^{2}(\Omega)}^{2}+ \\
+\left(8 \pi \eta^{-1}+\eta^{3} \sup _{\Omega,[0, T]} \mathcal{N}_{e}^{2}\left(\mathbf{x}^{\prime}, t\right)\right) \int_{0}^{T}\|\nabla \cdot \mathbf{E}(\cdot, t)\|_{L^{2}(\Omega)}^{2} d t+ \\
+\left(4 \pi \eta+2 \nu_{e} \eta^{2}+2 \eta\right) \int_{0}^{T}\|\nabla \cdot \mathbf{J}(\cdot, t)\|_{L^{2}(\Omega)}^{2} d t+ \\
+\eta^{3} \sup _{\Omega,[0, T]}\left(\nabla \mathcal{N}_{e}\left(\mathbf{x}^{\prime}, t\right)\right)^{2} \int_{0}^{T}\|\mathbf{E}(\cdot, t)\|_{L^{2}(\Omega)}^{2} d t+4 \pi \eta \int_{0}^{T}\left\|\partial_{t} \nabla \cdot \mathbf{P}(\cdot, t)\right\|_{L^{2}(\Omega)}^{2} d t .
\end{gathered}
$$

In addition to $(2.75)$, we recall that $\nabla \cdot \mathbf{B}\left(\mathbf{x}^{\prime}, t\right) \equiv 0$. According to Lemmas 2.1.4, 2.1.5, $\partial_{t} \mathbf{P} \in L^{\infty}\left(0, T ;\left(\mathcal{C}_{0}^{\infty}\right)^{3}\right)$ and $\mathcal{N}_{e}\left(\mathbf{x}^{\prime}, t\right) \in L^{\infty}\left(0, T ; \mathcal{C}_{0}^{\infty}\right)$, so using Grönwall's inequality we can confirm boundedness of the norms in the LHS of inequalities (2.74) and (2.75).

Similarly, using Lemma 2.1.7 and equations (2.70), (2.72), we can prove boundedness of the following combination of the norms:

$$
\begin{aligned}
& \left\|\partial_{t} \mathbf{E}(\cdot, T)\right\|_{\left(L^{2}(\Omega)\right)^{3}}^{2}+c^{2}\|\nabla \times \mathbf{E}(\cdot, T)\|_{\left(L^{2}(\Omega)\right)^{3}}^{2}+\left\|\partial_{t} \mathbf{B}(\cdot, T)\right\|_{\left(L^{2}(\Omega)\right)^{3}}^{2}+ \\
& \quad+c^{2}\|\nabla \times \mathbf{B}(\cdot, T)\|_{\left(L^{2}(\Omega)\right)^{3}}^{2}+\eta^{2}\left\{\left\|\partial_{t} \mathbf{J}(\cdot, T)\right\|_{\left(L^{2}(\Omega)\right)^{3}}^{2}+c^{2}\|\nabla \times \mathbf{J}(\cdot, T)\|_{\left(L^{2}(\Omega)\right)^{3}}^{2}\right\} .
\end{aligned}
$$

From here we deduce (2.73) .

Proof of Theorem 2.1. So far, we have proven that for all $T>0$, there exists a constant $C>0$ such that:

$$
\begin{array}{r}
\|\mathbf{E}\|_{L^{\infty}\left(0, T ;\left(H^{1}(\Omega)\right)^{3}\right) \cap H^{1}\left(0, T ;\left(L^{2}(\Omega)\right)^{3}\right)}^{2}+\|\mathbf{B}\|_{L^{\infty}\left(0, T ;\left(H^{1}(\Omega)\right)^{3}\right) \cap H^{1}\left(0, T ;\left(L^{2}(\Omega)\right)^{3}\right)}^{2}+ \\
\quad+\eta^{2}\|\mathbf{J}\|_{L^{\infty}\left(0, T ;\left(H^{1}(\Omega)\right)^{3}\right) \cap H^{1}\left(0, T ;\left(L^{2}(\Omega)\right)^{3}\right)}^{2}+\mu\|\bar{\psi}\|_{L^{\infty}\left(0, T ;\left(H^{1} \cap H_{1}\right)^{m}\right)} \leq C,
\end{array}
$$

where $\mu>0$ is a dimensional constant, such that $\operatorname{dim}(\mu)=\operatorname{dim}\left(\mathbf{E}^{2}\right)$. The boundness of the last term in the above inequality is a consequence of Lemma 2.1.1. Now as $L^{\infty}\left(0, T ;\left(H^{1}(\Omega)\right)^{3}\right) \times L^{\infty}\left(0, T ;\left(H^{1} \cap H_{1}\right)^{m}\right)$ is compactly embedded in $L^{2}(\Omega \times(0, T]) \times$ 
$\left(L^{2}\left(\mathbb{R}^{3}\right)\right)^{m}$ by Leray-Schauder's fixed point theorem we deduce the existence of a solution for (2.1), (2.2) and (2.5). The approach is the same as described in [124]. It is based on the introduction of a continuous mapping derived from (2.1), (2.2), which depends on a parameter $\lambda \in[0,1]$ and admits a fixed point in $L^{2}(\Omega \times(0, T]) \times$ $\left(L^{2}\left(\mathbb{R}^{3}\right)\right)^{m}$ as verifying Leray-Schauder's theorem assumptions [87].

Uniqueness is proven by a classical process of introducing two solutions of the Cauchy problem (2.1), (2.2): $\left(\mathbf{E}_{1}, \mathbf{B}_{1}, \mathbf{J}_{1}, \bar{\psi}_{1}\right)^{T}$ and $\left(\mathbf{E}_{2}, \mathbf{B}_{2}, \mathbf{J}_{2}, \bar{\psi}_{2}\right)^{T}$, and denoting their difference as $(\mathbf{E}, \mathbf{B}, \mathbf{J}, \bar{\psi})^{T}:=\left(\mathbf{E}_{2}-\mathbf{E}_{1}, \mathbf{B}_{2}-\mathbf{B}_{1}, \mathbf{J}_{2}-\mathbf{J}_{1}, \bar{\psi}_{2}-\bar{\psi}_{1}\right)^{T}$ with ICs $(\mathbf{E}(\cdot, 0), \mathbf{B}(\cdot, 0), \mathbf{J}(\cdot, 0), \bar{\psi}(\cdot, 0))^{T}=(\mathbf{0}, \mathbf{0}, \mathbf{0}, \mathbf{0})^{T}$. As in Lemmas 2.1 .6 and 2.1.1, see also for details [81], there exists a constant $C>0$ such that:

$$
\begin{aligned}
& \frac{d}{d t}\left\{\mu\|\bar{\psi}(t)\|_{\left(H^{1} \cap H_{1}\right)^{m}}^{2}+\int_{\Omega}\left(\left\|\mathbf{E}\left(\mathbf{x}^{\prime}, t\right)\right\|_{\left(L^{2}(\Omega)\right)^{3}}^{2}+\right.\right. \\
& \left.\left.+\left\|\mathbf{B}\left(\mathbf{x}^{\prime}, t\right)\right\|_{\left(L^{2}(\Omega)\right)^{3}}^{2}+\eta^{2}\left\|\mathbf{J}\left(\mathbf{x}^{\prime}, t\right)\right\|_{\left(L^{2}(\Omega)\right)^{3}}^{2}\right) d \mathbf{x}^{\prime}\right\} \leq \\
& C\left\{\mu\|\bar{\psi}(t)\|_{\left(H^{1} \cap H_{1}\right)^{m}}^{2}+\int_{\Omega}\left(\left\|\mathbf{E}\left(\mathbf{x}^{\prime}, t\right)\right\|_{\left(L^{2}(\Omega)\right)^{3}}^{2}+\right.\right. \\
& \left.\left.+\left\|\mathbf{B}\left(\mathbf{x}^{\prime}, t\right)\right\|_{\left(L^{2}(\Omega)\right)^{3}}^{2}+\eta^{2}\left\|\mathbf{J}\left(\mathbf{x}^{\prime}, t\right)\right\|_{\left(L^{2}(\Omega)\right)^{3}}^{2}\right) d \mathbf{x}^{\prime}\right\} .
\end{aligned}
$$

Using Grönwall's inequality we conclude $(\mathbf{E}(\cdot, t), \mathbf{B}(\cdot, t), \mathbf{J}(\cdot, t), \bar{\psi}(\cdot, t))^{T} \equiv(\mathbf{0}, \mathbf{0}, \mathbf{0}, \mathbf{0})^{T}$ for all $t>0$.

Moreover, the polarization is also unique, $\mathbf{P}=\mathbf{P}_{2}-\mathbf{P}_{1} \equiv \mathbf{0}$ for $t \geq 0$, as by definition:

$$
\begin{aligned}
& \mathbf{P}\left(\mathbf{x}^{\prime}, t\right)=\mathcal{N}\left(\mathbf{x}^{\prime}\right) \sum_{i=1}^{m} \mathbf{P}_{i}\left(\mathbf{x}^{\prime}, t\right)= \\
& =\mathcal{N}\left(\mathbf{x}^{\prime}\right) \sum_{i=1}^{m} \chi_{\Omega_{i}}\left(\mathbf{x}^{\prime}\right) \int_{\mathbb{R}^{3} \times \mathbb{R}_{+}} \mathbf{x}\left(\left|\psi_{i, 2}\left(R_{0}, \mathbf{x}, t\right)\right|^{2}-\left|\psi_{i, 1}\left(R_{0}, \mathbf{x}, t\right)\right|^{2}\right) d \mathbf{x} d R_{0},
\end{aligned}
$$

and as $\bar{\psi}(\cdot, t) \equiv \mathbf{0}$ for all $t>0$.

The main practical consequence of Theorem 2.1 in the sense of numerical treatment of the model is the conservation in time of regularity of the solutions, which 
justifies the use of finite difference methods (FDM) for solving Equations (2.1), (2.5) and $(2.2)[79,81]$. In addition we note that the FDM is a natural choice as the geometry of the computational domains of the MEs and TDSEs are simple enough.

\subsubsection{MASP Model Supplemented by Evolution Equations for Polarization and Free Electron Density}

This section discusses the issue, whether we could still rely on the same regularity of solutions in case of the MASP model enriched by the evolution equations for polarization and free electrons density. Certainly, it is too complicated to prove the theorem of existence and uniqueness for the MASP model with embedded general evolution equation (2.35) that, moreover, is nonlinear. However, since we intend to use this equation in the regime of relatively weak nonlinearity (with purpose to obtain a correction to the solution of homogeneous equation), it makes sense to consider at least the MASP model complemented by the homogeneous wave equations for polarization vector :

$$
\partial_{t}^{2} \mathbf{P}-v_{g}^{2} \triangle \mathbf{P}=0
$$

with velocity $v_{g}$, considered as a constant. Note that if we apply the operator $\nabla$ to the both sides of (2.77) and take into account that the divergence of the polarization vector equals to the density of the bound charges taken with opposite sign [70], $\nabla \cdot \mathbf{P}=-\left(\mathcal{N}-\mathcal{N}_{e}\right)$, we obtain the evolution equation for the free electron density:

$$
\partial_{t}^{2} \mathcal{N}_{e}-v_{g}^{2} \triangle \mathcal{N}_{e}=0
$$

while the afore-mentioned equation (2.29) describes one-directional wave only. To obtain (2.78), we recall that the gas density depends on spatial coordinate only, $\mathcal{N}=\mathcal{N}\left(\mathbf{x}^{\prime}\right)$, and we make an assumption that this dependence is close to constant, i.e. $\nabla \mathcal{N}\left(\mathbf{x}^{\prime}\right)=\mathbf{0}$ and $\triangle \mathcal{N}\left(\mathbf{x}^{\prime}\right)=0$ for all $\mathbf{x}^{\prime} \in \Omega$. 
Since we intend to solve the system of Equations (2.1), (2.2), (2.5), (2.77) and (2.78), we start from the initial data set $\left(\mathbf{E}_{0}, \mathbf{B}_{0}, \mathbf{J}_{0}, \psi_{0}, \mathbf{P}_{0}, \partial_{t} \mathbf{P}_{0}, \mathcal{N}_{e 0}, \partial_{t} \mathcal{N}_{e 0}\right)^{T}$. However, now the bounded domain of Maxwell's equations $\Omega$ contains only one "subdomain" $\widetilde{\Omega}$ with a gas, see the model represented in Sections 2.3.1 and 2.3.2, see Fig. 2.7. This means that currently $\psi_{0} \equiv \psi_{0,1}$, where the second subscript "1" refers to the initial (we note in advance that all the succeeding as well) values of the wavefunction relate to the "first cell" only. Using these microscopic data, we can compute $\left.\mathbf{P}(t)\right|_{\partial \tilde{\Omega}}$, see (2.2), and $\left.\mathcal{N}(t)\right|_{\partial \widetilde{\Omega}}$ see (2.9), (2.10), and their time derivatives in the first cell of the gas region, i.e. on the boundary of the gas region. We suppose that initially within the domain, the polarization vector and free electron number density equal zero: $\mathbf{P}_{0}=\mathbf{0} \in \mathbb{R}^{3}, \partial_{t} \mathbf{P}_{0}=\mathbf{0} \in \mathbb{R}^{3}, \mathcal{N}_{e 0}=0 \in \mathbb{R}, \partial_{t} \mathcal{N}_{e 0}=0 \in \mathbb{R}$. This provides for wave equations (2.77) and (2.78) the initial-boundary value problems. Now we state the existence theorem for the supplemented MASP model.

Theorem 2.2. Suppose that $\left(\mathbf{E}_{0}, \mathbf{B}_{0}, \mathbf{J}_{0}\right) \in\left(H^{1}(\Omega)\right)^{3} \times\left(H^{1}(\Omega)\right)^{3} \times\left(H^{1}(\Omega)\right)^{3}, \psi_{0} \in$ $\left(H^{1} \cap H_{1}\right), R_{0}>0$ is a constant for all $t \in \mathbb{R}_{+}$and $\mathcal{N} \in C_{0}^{\infty}(\Omega)$. Suppose that on the smooth boundary $\partial \Omega$ zero Dirichlet $B C$ s are imposed for vectors $\mathbf{E}, \mathbf{B}, \mathbf{J}, \mathbf{P}$ for all $t \in \mathbb{R}_{+}$. In addition, on the smooth boundary $\partial \widetilde{\Omega}$ of the gas region the values of $\left.\mathbf{P}(t)\right|_{\partial \widetilde{\Omega}},\left.\mathcal{N}_{e}(t)\right|_{\partial \widetilde{\Omega}}$ are computed via $\psi\left(R_{0}, \mathbf{x}, t\right)$ from (2.2) and (2.9), (2.10) with $m=$ 1 , while for $t=0\left(\mathbf{P}_{0}, \partial_{t} \mathbf{P}_{0}, \mathcal{N}_{e 0}, \partial_{t} \mathcal{N}_{e 0}\right)=(\mathbf{0}, \mathbf{0}, 0,0)$. Then there exists a time $T>0$, and a unique solution $(\mathbf{E}, \mathbf{B}, \mathbf{J}, \mathbf{P}, \psi) \in\left(L^{\infty}\left(0, T ;\left(H^{1}(\Omega)\right)^{3}\right) \cap H^{1}\left(0, T ;\left(L^{2}(\Omega)\right)^{3}\right)\right)^{4} \times$ $L^{\infty}\left(0, T ;\left(H^{1} \cap H_{1}\right)\right)$ to Equations (2.1), (2.2) (with $\left.m=1\right)$, (2.5), (2.77) and (2.78).

In the case of the MASP model supplemented by the evolution equations (2.77) and (2.78), we first need to adjust several lemmas proven above. In particular, Lemmas 2.1.3, 2.1.4 are still true for $\mathbf{x}^{\prime}=\mathbf{x}_{1}^{\prime}$, i.e. in the first gas "cell" only. However, inside the gas subdomain we have to consider the evolution equations, in particular

$$
\partial_{t}^{2} \mathbf{P}=v_{g}^{2} \triangle \mathbf{P}
$$


Lemma 2.2.1. We assume Dirichlet $B C s$ on $\partial \Omega$ : $\left.\mathbf{P}(t)\right|_{\partial \Omega}=\mathbf{0} \in \mathbb{R}^{3}$, then for all $T>0$, and $\Omega \subset \mathbb{R}^{3}$

$$
\begin{aligned}
& \left\|\partial_{t} \mathbf{P}(\cdot, T)\right\|_{\left(L^{2}(\Omega)\right)^{3}}^{2}+v_{g}^{2}\|\nabla \times \mathbf{P}(\cdot, T)\|_{\left(L^{2}(\Omega)\right)^{3}}^{2}=\left\|\left(\partial_{t} \mathbf{P}\right)_{0}(\cdot)\right\|_{\left(L^{2}(\Omega)\right)^{3}}^{2}+ \\
& \quad+v_{g}^{2}\left\|(\nabla \times \mathbf{P})_{0}(\cdot)\right\|_{\left(L^{2}(\Omega)\right)^{3}}^{2}+2 v_{g}^{2} \int_{0}^{T} \int_{\Omega} \partial_{t} \mathbf{P}\left(\mathbf{x}^{\prime}, t\right) \cdot \nabla \mathcal{N}_{e}\left(\mathbf{x}^{\prime}, t\right) d \mathbf{x}^{\prime} d t
\end{aligned}
$$

Proof. We multiply (2.79) by $\partial_{t} \mathbf{P}$ and consider separately the LHS and the RHS:

$$
\begin{aligned}
\partial_{t} \mathbf{P} \cdot \partial_{t}^{2} \mathbf{P} & =\frac{1}{2} \partial_{t}\left|\partial_{t} \mathbf{P}\right|^{2} \\
\partial_{t} \mathbf{P} \cdot \triangle \mathbf{P} & =\left(\partial_{t} \mathbf{P} \cdot \nabla\right)(\nabla \cdot \mathbf{P})-\partial_{t} \mathbf{P} \cdot[\nabla \times[\nabla \times \mathbf{P}]]
\end{aligned}
$$

where $\left|\partial_{t} \mathbf{P}\right|^{2}=\partial_{t} \mathbf{P} \cdot \partial_{t} \mathbf{P}$.

Recall that $\nabla \cdot \mathbf{P}=-\left(\mathcal{N}-\mathcal{N}_{e}\right)$, so the first term of the RHS reads

$$
\left(\partial_{t} \mathbf{P} \cdot \nabla\right)(\nabla \cdot \mathbf{P})=-\left(\partial_{t} \mathbf{P} \cdot \nabla\right)\left(\mathcal{N}-\mathcal{N}_{e}\right)=\partial_{t} \mathbf{P} \cdot \nabla \mathcal{N}_{e}
$$

The second term in $(2.82)$ can be rewritten

$$
\begin{gathered}
\partial_{t} \mathbf{P} \cdot[\nabla \times[\nabla \times \mathbf{P}]]=\nabla \cdot\left[[\nabla \times \mathbf{P}] \times \partial_{t} \mathbf{P}\right]+[\nabla \times \mathbf{P}] \cdot\left[\nabla \times \partial_{t} \mathbf{P}\right]= \\
=\nabla \cdot\left[[\nabla \times \mathbf{P}] \times \partial_{t} \mathbf{P}\right]+\frac{1}{2} \partial_{t}|\nabla \times \mathbf{P}|^{2},
\end{gathered}
$$

so finally we come to

$$
\partial_{t}\left\{\left|\partial_{t} \mathbf{P}\right|^{2}+v_{g}^{2}|\nabla \times \mathbf{P}|^{2}\right\}=2 v_{g}^{2} \partial_{t} \mathbf{P} \cdot \nabla \mathcal{N}_{e}-2 v_{g}^{2} \nabla \cdot\left[[\nabla \times \mathbf{P}] \times \partial_{t} \mathbf{P}\right]
$$

Now we integrate $(2.83)$ over $(0, T) \times \Omega$ (applying the divergence theorem and using the BCs) to obtain (2.80).

Similarly, in addition to Lemma 2.1 .5 we need to consider the property of $\mathcal{N}_{e}$ inside the subdomain. 
Lemma 2.2.2. We assume Dirichlet $B C s$ on $\partial \Omega:\left.\mathcal{N}_{e}(t)\right|_{\partial \Omega}=0 \in \mathbb{R}$, then for all $T>0$ and $\Omega \subset \mathbb{R}^{3}$

$$
\left\|\partial_{t} \mathcal{N}_{e}(\cdot, T)\right\|_{L^{2}(\Omega)}^{2}+v_{g}^{2}\left\|\nabla \mathcal{N}_{e}(\cdot, T)\right\|_{L^{2}(\Omega)}^{2}=\left\|\left(\partial_{t} \mathcal{N}_{e}\right)_{0}(\cdot)\right\|_{L^{2}(\Omega)}^{2}+v_{g}^{2}\left\|\left(\nabla \mathcal{N}_{e}\right)_{0}(\cdot)\right\|_{L^{2}(\Omega)}^{2}
$$

Proof. We multiply the wave equation (2.78) by $\partial_{t} \mathcal{N}_{e}$ and note that

$$
\begin{aligned}
\partial_{t} \mathcal{N}_{e} \partial_{t}^{2} \mathcal{N}_{e} & =\frac{1}{2}\left(\partial_{t} \mathcal{N}_{e}\right)^{2} \\
\partial_{t} \mathcal{N}_{e} \triangle \mathcal{N}_{e} & =\nabla \cdot\left(\partial_{t} \mathcal{N}_{e} \nabla \mathcal{N}_{e}\right)-\left(\nabla \mathcal{N}_{e}\right) \cdot\left(\nabla \partial_{t} \mathcal{N}_{e}\right)= \\
& =\nabla \cdot\left(\partial_{t} \mathcal{N}_{e} \nabla \mathcal{N}_{e}\right)-\frac{1}{2} \partial_{t}\left(\nabla \mathcal{N}_{e}\right)^{2}
\end{aligned}
$$

Then we integrate both sides over $(0, T) \times \Omega$ to $(2.84)$.

Lemma 2.2.3. There exists a constant $C_{1}>0$, such that for all $T>0$

$$
\sup _{0 \leq t \leq T}\left\|\mathcal{N}_{e}(\cdot, t)\right\|_{H^{1}(\Omega)}^{2} \leq C_{1}
$$

Proof. From $\partial_{t} \mathcal{N}_{e}^{2}=2 \mathcal{N}_{e} \partial_{t} \mathcal{N}_{e}$ we deduce

$$
\begin{aligned}
\left\|\mathcal{N}_{e}(\cdot, T)\right\|_{L^{2}(\Omega)}^{2} \leq\left\|\mathcal{N}_{e 0}\right\|_{L^{2}(\Omega)}^{2} & +\eta^{-1} \int_{0}^{T}\left\|\mathcal{N}_{e}(\cdot, T)\right\|_{L^{2}(\Omega)}^{2} d t+ \\
& +\eta \int_{0}^{T}\left\|\partial_{t} \mathcal{N}_{e}(\cdot, T)\right\|_{L^{2}(\Omega)}^{2} d t
\end{aligned}
$$

From now on in current section, $\eta$ is defined in the same way as in Remark 2, see p. 94. Then from $(2.88),(2.84)$ and Grönwall's inequality we come to (2.87).

Lemma 2.2.4. There exists a constant $C_{2}>0$ such that for all $T>0$

$$
\sup _{0 \leq t \leq T}\|\mathbf{P}(\cdot, t)\|_{\left(H^{1}(\Omega)\right)^{3}}^{2} \leq C_{2}
$$


Proof. From Lemmas 2.2.1 and for all $t>0$

$$
\begin{array}{r}
\left\|\partial_{t} \mathbf{P}(\cdot, T)\right\|_{\left(L^{2}(\Omega)\right)^{3}}^{2}+v_{g}^{2}\|\nabla \times \mathbf{P}(\cdot, T)\|_{\left(L^{2}(\Omega)\right)^{3}}^{2} \leq\left\|\left(\partial_{t} \mathbf{P}\right)_{0}\right\|_{\left(L^{2}(\Omega)\right)^{3}}^{2}+ \\
+v_{g}^{2}\left\|(\nabla \times \mathbf{P})_{0}\right\|_{\left(L^{2}(\Omega)\right)^{3}}^{2}+\eta^{-1} \int_{0}^{T}\left\|\partial_{t} \mathbf{P}(\cdot, t)\right\|_{\left(L^{2}(\Omega)\right)^{3}}^{2} d t+ \\
+\eta v_{g}^{4} \int_{0}^{T}\left\|\nabla \mathcal{N}_{e}(\cdot, t)\right\|_{L^{2}(\Omega)}^{2} d t .
\end{array}
$$

Thus, we can conclude by Grönwall's inequality and (2.87) that $\left\|\partial_{t} \mathbf{P}(\cdot, T)\right\|_{\left(L^{2}(\Omega)\right)^{3}}^{2}$ and $\|\nabla \times \mathbf{P}(\cdot, T)\|_{\left(L^{2}(\Omega)\right)^{3}}^{2}$ are bounded. We recall that according Lemma 2.2.3 and relation $\nabla \cdot \mathbf{P}=-\left(\mathcal{N}-\mathcal{N}_{e}\right)$, norm $\|\nabla \cdot \mathbf{P}(\cdot, T)\|_{L^{2}(\Omega)}^{2}$ is bounded as well. Hence from $\partial_{t} \mathbf{P}^{2}=\mathbf{P} \partial_{t} \mathbf{P}$ we obtain

$$
\begin{aligned}
\|\mathbf{P}(\cdot, T)\|_{\left(L^{2}(\Omega)\right)^{3}}^{2} & \leq\left\|\mathbf{P}_{0}\right\|_{\left(L^{2}(\Omega)\right)^{3}}^{2}+ \\
& +\eta^{-1} \int_{0}^{T}\|\mathbf{P}(\cdot, t)\|_{\left(L^{2}(\Omega)\right)^{3}}^{2} d t+\eta \int_{0}^{T}\left\|\partial_{t} \mathbf{P}(\cdot, t)\right\|_{\left(L^{2}(\Omega)\right)^{3}}^{2} d t,
\end{aligned}
$$

thus, again with Grönwall's inequality and (2.90) we deduce (2.89).

Note that from Lemma 2.2.3 we deduce boundedness of $\left\|\partial_{t}(\nabla \cdot \mathbf{P})(\cdot, T)\right\|_{L^{2}(\Omega)}^{2}=$ $\left\|\partial_{t} \mathcal{N}_{e}(\cdot, T)\right\|_{L^{2}(\Omega)}^{2}$ and then deduce (2.89). Thus, all needed conditions to prove Lemma 2.1.9 are satisfied. Now we can extend Lemma 2.1.9 in the case of the supplemented MASP model.

Lemma 2.2.5. There exists a constant $C>0$ such that for all $T>0$

$$
\begin{aligned}
\sup _{0 \leq t \leq T} & \|\mathbf{E}(\cdot, t)\|_{\left(H^{1}(\Omega)\right)^{3}}^{2}+\sup _{0 \leq t \leq T}\|\mathbf{B}(\cdot, t)\|_{\left(H^{1}(\Omega)\right)^{3}}^{2}+ \\
& +\eta^{2} \sup _{0 \leq t \leq T}\|\mathbf{J}(\cdot, t)\|_{\left(H^{1}(\Omega)\right)^{3}}^{2}+\sup _{0 \leq t \leq T}\|\mathbf{P}(\cdot, t)\|_{\left(H^{1}(\Omega)\right)^{3}}^{2} \leq C .
\end{aligned}
$$

Proof. We can deduce this result from Lemmas 2.1.9 and 2.2.4.

Proof of Theorem 2.2. As in the proof of theorem 2.1, the existence is based on Leray-Schauder's fixed point theorem [87]. In Lemmas 2.1.6-2.1.8 and Lemmas 2.2.1- 
2.2.3, we proved that for all $T>0$ there exists a constant $C>0$ such that:

$$
\begin{gathered}
\|\mathbf{E}\|_{L^{\infty}\left(0, T ;\left(H^{1}(\Omega)\right)^{3}\right) \cap H^{1}\left(0, T ;\left(L^{2}(\Omega)\right)^{3}\right)}^{2}+\|\mathbf{B}\|_{L^{\infty}\left(0, T ;\left(H^{1}(\Omega)\right)^{3}\right) \cap H^{1}\left(0, T ;\left(L^{2}(\Omega)\right)^{3}\right)}^{2}+ \\
+\eta^{2}\|\mathbf{J}\|_{L^{\infty}\left(0, T ;\left(H^{1}(\Omega)\right)^{3}\right) \cap H^{1}\left(0, T ;\left(L^{2}(\Omega)\right)^{3}\right)}^{2}+\|\mathbf{P}\|_{L^{\infty}\left(0, T ;\left(H^{1}(\Omega)\right)^{3}\right) \cap H^{1}\left(0, T ;\left(L^{2}(\Omega)\right)^{3}\right)^{2}}^{2}+ \\
+\mu\|\psi\|_{L^{\infty}\left(0, T ;\left(H^{1} \cap H_{1}\right)\right)} \leq C,
\end{gathered}
$$

and $L^{\infty}\left(0, T ;\left(H^{1}(\Omega)\right)^{3}\right) \times L^{\infty}\left(0, T ;\left(H^{1} \cap H_{1}\right)\right)$ is compactly embedded in $L^{2}(\Omega \times$ $(0, T]) \times\left(L^{2}\left(\mathbb{R}^{3} \times \mathbb{R}_{+}\right)\right)$. The approach follows [124]. We introduce a continuous mapping derived from $(2.1),(2.2),(2.5),(2.77)$, and (2.78), that depends on a parameter $\lambda \in[0,1]$ and that admits a fixed point in $L^{2}(\Omega \times(0, T]) \times\left(L^{2}\left(\mathbb{R}^{3} \times \mathbb{R}_{+}\right)\right)$as verifying Leray-Schauder's theorem assumptions.

Uniqueness is proven to be a usual process: we take the difference vector $(\mathbf{E}, \mathbf{B}, \mathbf{J}, \bar{\psi})^{T}:=$ $\left(\mathbf{E}_{2}-\mathbf{E}_{1}, \mathbf{B}_{2}-\mathbf{B}_{1}, \mathbf{J}_{2}-\mathbf{J}_{1}, \mathbf{P}_{2}-\mathbf{P}_{1}, \bar{\psi}_{2}-\bar{\psi}_{1}\right)^{T}$, with $\operatorname{ICs}(\mathbf{E}(\cdot, 0), \mathbf{B}(\cdot, 0), \mathbf{J}(\cdot, 0), \mathbf{P}(\cdot, 0) \bar{\psi}(\cdot, 0))^{T}=$ $(\mathbf{0}, \mathbf{0}, \mathbf{0}, \mathbf{0})^{T}$, where $\left(\mathbf{E}_{1}, \mathbf{B}_{1}, \mathbf{J}_{1}, \mathbf{P}_{1}, \bar{\psi}_{1}\right)^{T}$ and $\left(\mathbf{E}_{2}, \mathbf{B}_{2}, \mathbf{J}_{2}, \mathbf{P}_{2}, \bar{\psi}_{2}\right)^{T}$ denote two solutions. Then applying the methods presented in the above lemmas, see also [81], we come to the inequality with some constant $C>0$ :

$$
\begin{gathered}
\frac{d}{d t}\left\{\mu\|\bar{\psi}(t)\|_{\left(H^{1} \cap H_{1}\right)}^{2}+\int_{\Omega}\left(\left\|\mathbf{E}\left(\mathbf{x}^{\prime}, t\right)\right\|_{\left(L^{2}(\Omega)\right)^{3}}^{2}+\left\|\mathbf{B}\left(\mathbf{x}^{\prime}, t\right)\right\|_{\left(L^{2}(\Omega)\right)^{3}}^{2}\right.\right. \\
\left.+\eta^{2}\left\|\mathbf{J}\left(\mathbf{x}^{\prime}, t\right)\right\|_{\left(L^{2}(\Omega)\right)^{3}}^{2}+\left\|\mathbf{P}\left(\mathbf{x}^{\prime}, t\right)\right\|_{\left(L^{2}(\Omega)\right)^{3}}^{2} d \mathbf{x}^{\prime}\right\} \leq \\
C\left\{\mu\|\bar{\psi}(t)\|_{\left(H^{1} \cap H_{1}\right)}^{2}+\int_{\Omega}\left(\left\|\mathbf{E}\left(\mathbf{x}^{\prime}, t\right)\right\|_{\left(L^{2}(\Omega)\right)^{3}}^{2}+\left\|\mathbf{B}\left(\mathbf{x}^{\prime}, t\right)\right\|_{\left(L^{2}(\Omega)\right)^{3}}^{2}+\right.\right. \\
\left.+\eta^{2}\left\|\mathbf{J}\left(\mathbf{x}^{\prime}, t\right)\right\|_{\left(L^{2}(\Omega)\right)^{3}}^{2}+\left\|\mathbf{P}\left(\mathbf{x}^{\prime}, t\right)\right\|_{\left(L^{2}(\Omega)\right)^{3}}^{2} d \mathbf{x}^{\prime}\right\} .
\end{gathered}
$$

We conclude by Grönwall's inequality that $(\mathbf{E}(\cdot, t), \mathbf{B}(\cdot, t), \mathbf{J}(\cdot, t), \mathbf{P}(\cdot, t), \bar{\psi}(\cdot, t))^{T} \equiv$ $(\mathbf{0}, \mathbf{0}, \mathbf{0}, \mathbf{0})^{T}$ for all $t>0$.

Summarizing Section 2.4, because of the regularity of the solutions not only of the "pure" MASP model, but the "extended" MASP model as well, in the next Chapter we proceed to the description of respective finite difference schemes. 


\section{Chapter 3}

\section{Numerical Methods for Solving the MASP Model}

In this chapter we describe and analyze the numerical schemes used to approximate the MASP equations, and the polarization evolution equation in $1 \mathrm{~d}-2 \mathrm{~d}$, and $3 \mathrm{~d}-2 \mathrm{~d}$ geometries.

\subsection{Discretization of the 1d Maxwell Equations}

In the case of $1 \mathrm{~d}$ MEs, the components of EM field $\mathbf{E}\left(\mathbf{x}^{\prime}, t\right)$ and $\mathbf{B}\left(\mathbf{x}^{\prime}, t\right)$ are functions of the $z^{\prime}$-coordinate, and time only:

$$
\left\{\begin{array}{l}
\mathbf{E}\left(\mathbf{x}^{\prime}, t\right)=\left(E_{x^{\prime}}\left(z^{\prime}, t\right), E_{y^{\prime}}\left(z^{\prime}, t\right)\right)^{T} \\
\mathbf{B}\left(\mathbf{x}^{\prime}, t\right)=\left(B_{x^{\prime}}\left(z^{\prime}, t\right), B_{y^{\prime}}\left(z^{\prime}, t\right)\right)^{T}
\end{array}\right.
$$

We start from the first pair of the MEs (2.1):

$$
\left\{\begin{array}{l}
\partial_{t} \mathbf{E}\left(z^{\prime}, t\right)=c \nabla \times \mathbf{B}\left(z^{\prime}, t\right)-4 \pi \partial_{t} \mathbf{P}\left(z^{\prime}, t\right), \quad z^{\prime} \in \Omega, t>0 \\
\partial_{t} \mathbf{B}\left(z^{\prime}, t\right)=-c \nabla \times \mathbf{E}\left(z^{\prime}, t\right)
\end{array}\right.
$$


with the domain $\Omega \subset \mathbb{R}$ consisting of the following regions $L_{1}, L$ and $L_{2}$, see illustrations in Figs. 2.2 and 2.5. The initial laser pulse is in the form of envelope (2.11), which can be either Gaussian see Fig. 2.3, or a $\sin ^{2}$-envelope, see Fig. 2.4. First, we rewrite (3.2) by components

$$
\left\{\begin{array}{l}
\partial_{t} E_{x^{\prime}}\left(z^{\prime}, t\right)=-c \partial_{z^{\prime}} B_{y^{\prime}}\left(z^{\prime}, t\right)-4 \pi \partial_{t} P_{x^{\prime}}\left(z^{\prime}, t\right) \\
\partial_{t} E_{y^{\prime}}\left(z^{\prime}, t\right)=\quad c \partial_{z^{\prime}} B_{x^{\prime}}\left(z^{\prime}, t\right)-4 \pi \partial_{t} P_{y^{\prime}}\left(z^{\prime}, t\right) \\
\partial_{t} B_{x^{\prime}}\left(z^{\prime}, t\right)=\quad c \partial_{z^{\prime}} E_{y^{\prime}}\left(z^{\prime}, t\right) \\
\partial_{t} B_{y^{\prime}}\left(z^{\prime}, t\right)=-c \partial_{z^{\prime}} E_{x^{\prime}}\left(z^{\prime}, t\right)
\end{array}\right.
$$

It can be seen that we have 2 separate pairs of PDEs, for $\left(E_{x^{\prime}}, B_{y^{\prime}}\right)$ and $\left(E_{y^{\prime}}, B_{x^{\prime}}\right)$. These equations could be also rewritten for each component of the EM field:

$$
\left\{\begin{array}{l}
\partial_{t}^{2} E_{x^{\prime}}\left(z^{\prime}, t\right)=c^{2} \partial_{z^{\prime}}^{2} E_{x^{\prime}}\left(z^{\prime}, t\right)-4 \pi \partial_{t}^{2} P_{x^{\prime}}\left(z^{\prime}, t\right), \\
\partial_{t}^{2} E_{y^{\prime}}\left(z^{\prime}, t\right)=c^{2} \partial_{z^{\prime}}^{2} E_{y^{\prime}}\left(z^{\prime}, t\right)-4 \pi \partial_{t}^{2} P_{y^{\prime}}\left(z^{\prime}, t\right), \\
\partial_{t}^{2} B_{x^{\prime}}\left(z^{\prime}, t\right)=c^{2} \partial_{z^{\prime}}^{2} B_{x^{\prime}}\left(z^{\prime}, t\right)-4 c \pi \partial_{t z^{\prime}} P_{y^{\prime}}\left(z^{\prime}, t\right), \\
\partial_{t}^{2} B_{y^{\prime}}\left(z^{\prime}, t\right)=c^{2} \partial_{z^{\prime}}^{2} B_{y^{\prime}}\left(z^{\prime}, t\right)+4 c \pi \partial_{t z^{\prime}} P_{x^{\prime}}\left(z^{\prime}, t\right) .
\end{array}\right.
$$

The system (3.4) represents 4 nonhomogeneous wave equations, where the terms with 2nd order partial derivatives of the polarization vector model the "exciting force".

With the aim of the specific physical applications, we used 2 numerical schemes to discretize the 1d Maxwell's equations. The first scheme, which is of first-order in time and space (as is proven in Subsection 3.1), was employed to compute the high harmonics spectra resulting from LP- and CP-laser pulses propagation in a molecular gas based on the pure MASP model. The second scheme, being more precise in time, was used to implement the MASP model enriched by the polarization evolution equation. 


\subsubsection{Numerical Scheme I}

We now present the Lax-Friedrichs [40,49,101,113] finite difference scheme to solve (3.3). Let us denote the space step in $z^{\prime}$ by $h=\left(L_{1}+L+L_{2}\right) / N$, where $N \geq 1$. Then $z^{\prime}{ }_{i}=i h(N \geq i \geq 0)$. Next, we denote by $\tau$ the time step, and by $t_{n}=n \tau$ $(n \geq 0)$ the discrete times. On that mesh, under certain smoothness assumptions on the solution of the problem, we designate $\left(E_{x^{\prime}}\right)_{i}^{n} \approx E_{x^{\prime}}\left(z^{\prime}{ }_{i}, t_{n}\right),\left(E_{y^{\prime}}\right)_{i}^{n} \approx E_{y^{\prime}}\left(z^{\prime}{ }_{i}, t_{n}\right)$, $\left(B_{x^{\prime}}\right)_{i}^{n} \approx B_{x^{\prime}}\left(z^{\prime}{ }_{i}, t_{n}\right),\left(B_{y^{\prime}}\right)_{i}^{n} \approx B_{y^{\prime}}\left(z^{\prime}{ }_{i}, t_{n}\right)$. For convenience, we introduce a vector function $\mathbf{U}: \mathbb{R} \times \mathbb{R}_{+} \rightarrow \mathbb{R}^{4}$ as

$$
\mathbf{U}\left(z^{\prime}, t\right)=\left(E_{x^{\prime}}\left(z^{\prime}, t\right), E_{y^{\prime}}\left(z^{\prime}, t\right), B_{x^{\prime}}\left(z^{\prime}, t\right), B_{y^{\prime}}\left(z^{\prime}, t\right)\right)^{T}
$$

and $\mathbf{U}_{i}^{n} \approx \mathbf{U}\left(z^{\prime}{ }_{i}, t_{n}\right)$. With such notation, we use the following approximation to discretize the partial derivatives desired in (3.3)

$$
\begin{aligned}
\left(\partial_{t} \mathbf{U}\right)_{i}^{n} \equiv \partial_{t} \mathbf{U}\left(z^{\prime}{ }_{i}, t_{n}\right) \approx \frac{1}{\tau}\left[\mathbf{U}_{i}^{n+1}-\frac{1}{2}\left(\mathbf{U}_{i+1}^{n}+\mathbf{U}_{i-1}^{n}\right)\right], \\
\left(\partial_{z^{\prime}} \mathbf{U}\right)_{i}^{n} \equiv \partial_{z^{\prime}} \mathbf{U}\left(z^{\prime}{ }_{i}, t_{n}\right) \approx \frac{1}{2 h}\left(\mathbf{U}_{i+1}^{n}-\mathbf{U}_{i-1}^{n}\right) .
\end{aligned}
$$

It corresponds to the difference stencil shown on Fig. 3.1, and resulting in the finite difference scheme:

$$
\left\{\begin{array}{l}
\frac{2\left(E_{x^{\prime}}\right)_{i}^{n+1}-\left(E_{x^{\prime}}\right)_{i+1}^{n}-\left(E_{x^{\prime}}\right)_{i-1}^{n}}{2 \tau}=-c \frac{\left(B_{y^{\prime}}\right)_{i+1}^{n}-\left(B_{y^{\prime}}\right)_{i-1}^{n}}{2 h}-4 \pi\left(\partial_{t} P_{x^{\prime}}\right)_{i}^{n}, \\
\frac{2\left(E_{y^{\prime}}\right)_{i}^{n+1}-\left(E_{y^{\prime}}\right)_{i+1}^{n}-\left(E_{y^{\prime}}\right)_{i-1}^{n}}{2 \tau}=c \frac{\left(B_{x^{\prime}}\right)_{i+1}^{n}-\left(B_{x^{\prime}}\right)_{i-1}^{n}}{2 h}-4 \pi\left(\partial_{t} P_{y^{\prime}}\right)_{i}^{n}, \\
\frac{2\left(B_{x^{\prime}}\right)_{i}^{n+1}-\left(B_{x^{\prime}}\right)_{i+1}^{n}-\left(B_{x^{\prime}}\right)_{i-1}^{n}}{2 \tau}=c \frac{\left(E_{y^{\prime}}\right)_{i+1}^{n}-\left(E_{y^{\prime}}\right)_{i-1}^{n}}{2 h}, \\
\frac{2\left(B_{y^{\prime}}\right)_{i}^{n+1}-\left(B_{y^{\prime}}\right)_{i+1}^{n}-\left(B_{y^{\prime}}\right)_{i-1}^{n}}{2 \tau}=-c \frac{\left(E_{x^{\prime}}\right)_{i+1}^{n}-\left(E_{x^{\prime}}\right)_{i-1}^{n}}{2 h},
\end{array}\right.
$$

where the partial derivatives $\left(\partial_{t} P_{x^{\prime}}\right)_{i}^{n}$ and $\left(\partial_{t} P_{y^{\prime}}\right)_{i}^{n}$ equal 0 for the vacuum regions: $i \in\left\{0,1, \ldots I_{1}\right\} \cup\left\{I_{2}, I_{2}+1, \ldots N\right\}$, with $I_{2}>I_{1}$. For the gas region, where number density is equal to $\mathcal{N}_{0}$, and $i \in\left\{I_{1}, I_{1}+1, \ldots I_{2}\right\}$, these time derivatives are obtained 


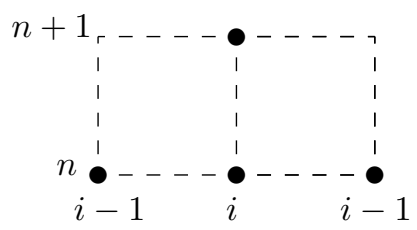

FIGURE 3.1: 4-point stencil for the discretization (3.6)

by solving the Schrödinger equations in each respective Maxwell's cell: $\left(\partial_{t} P_{x^{\prime}}\right)_{i}^{n} \approx$ $\mathcal{N}_{0}\left(\partial_{t} d_{x^{\prime}}\right)_{i}^{n},\left(\partial_{t} P_{y^{\prime}}\right)_{i}^{n} \approx \mathcal{N}_{0}\left(\partial_{t} d_{y^{\prime}}\right)_{i}^{n}$

Thus, on each time step the electromagnetic field components reads

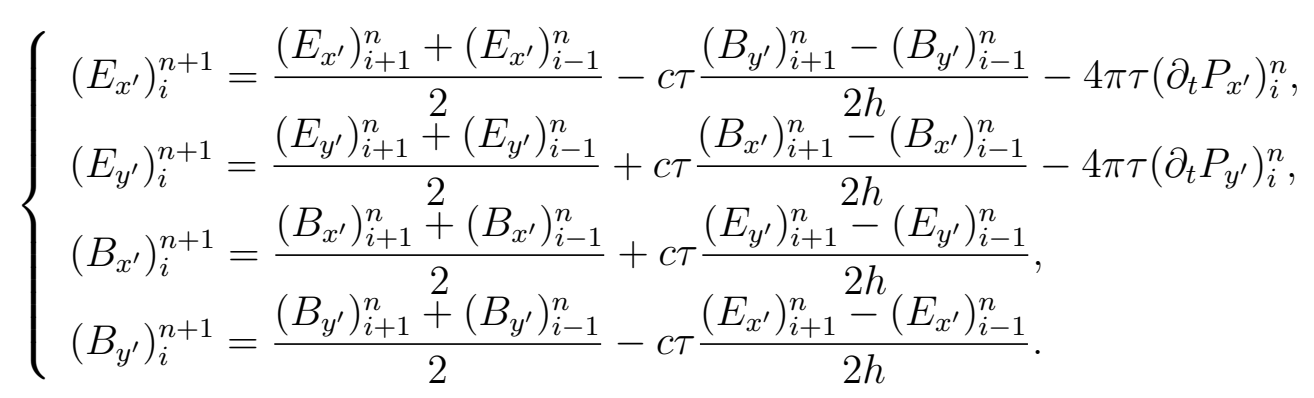

We use this Finite Difference Scheme (FDS) to compute $\left\{\mathbf{U}_{i}^{n}\right\}$ for $N-1 \geq i \geq 1$ and $n>0$, with Dirichlet conditions on the boundaries of the computational domain (recall that a wave propagates in the $z^{\prime}$-direction):

$$
\mathbf{U}_{0}^{n}=\mathbf{0}, \quad \mathbf{U}_{N}^{n}=\mathbf{U}_{N}^{n-1} \quad \text { for all } n \geq 1
$$

Further we consider the issues of consistency, stability and convergence $[49,101]$ of the FDS (3.8).

Proposition 3.1 (Consistency of Scheme I). For $h \neq c \tau$, the truncation error of Scheme $I(3.8)$ is $\mathcal{O}\left(\tau+h^{2} / \tau\right)$.

Proof. We rewrite the system (3.3) in the form $\mathcal{P} \mathbf{U}=\mathbf{F}$, where the vector $\mathbf{U}$ is defined according to (3.5), the vector

$$
\mathbf{F}=\left(-4 \pi \partial_{t} P_{x^{\prime}}\left(z^{\prime}, t\right),-4 \pi \partial_{t} P_{y^{\prime}}\left(z^{\prime}, t\right), 0,0\right)^{T}
$$


and the linear differential operator $\mathcal{P}$ :

$$
\mathcal{P}=\mathbf{I}_{4} \partial_{t}+c \mathbf{J}_{4} \partial_{z^{\prime}}
$$

where $\mathbf{I}_{4}$ is the $4 \times 4$ identity matrix, while the following matrix $\mathbf{J}_{4}$ is defined as

$$
\mathbf{J}_{4}=\left[\begin{array}{cccc}
0 & 0 & 0 & 1 \\
0 & 0 & -1 & 0 \\
0 & -1 & 0 & 0 \\
1 & 0 & 0 & 0
\end{array}\right] \text {. }
$$

We denote by $\mathcal{P}_{\tau, h}$ the discrete operator approximating $\mathcal{P}$, so we get in accordance with the FDS (3.7) and (3.6),

$$
\left(\mathcal{P}_{\tau, h} \mathbf{U}\right)_{i}^{n}=\frac{1}{2 \tau} \mathbf{I}_{4}\left(2 \mathbf{U}_{i}^{n+1}-\mathbf{U}_{i+1}^{n}-\mathbf{U}_{i-1}^{n}\right)+\frac{c}{2 h} \mathbf{J}_{4}\left(\mathbf{U}_{i+1}^{n}-\mathbf{U}_{i-1}^{n}\right),
$$

Thus, $\mathcal{P}_{\tau, h} \mathbf{U}=\mathbf{F}_{\tau, h}$, where $\mathbf{F}_{\tau, h}$ is an appropriate discretization of $\mathbf{F}$.

Now to prove the pointwise consistence of the FDS, we should show that for any $\Phi \in \mathbb{R}^{4}$, smooth enough solution to (3.3), we have

$$
\mathcal{P}_{\tau, h} \Phi-\mathcal{P} \Phi-\left(\mathbf{F}_{\tau, h}-\mathbf{F}\right) \rightarrow \mathbf{0} \in \mathbb{R}^{4} \quad \text { as } \quad(\tau, h) \rightarrow(0,0)
$$

In addition, to determine the order of consistency of the FDS in space $p$ and time $q$, we should prove that

$$
\mathcal{P}_{\tau, h} \Phi-\mathcal{P} \Phi-\left(\mathbf{F}_{\tau, h}-\mathbf{F}\right)=\mathbf{1}\left(\mathcal{O}\left(h^{p}\right)+\mathcal{O}\left(\tau^{q}\right)\right), \text { as }(\tau, h) \rightarrow(0,0)
$$

where $(p, q) \in[1, \infty)$ and $\mathbf{1} \in \mathbb{R}^{4}$.

We start with determining the order of consistency of the discretization of $\mathcal{P}_{\tau, h}$, in 
other words for the FDS corresponding to the homogeneous equation $\mathcal{P} \mathbf{F}=\mathbf{0}$. First, with (3.10) it follows for $\Phi$ that

$$
\mathbf{I}_{4} \partial_{t} \Phi=-\mathbf{J}_{4} c \partial_{z^{\prime}} \Phi
$$

Also in that case the system (3.4) reduces to

$$
\partial_{t}^{2} \Phi=c^{2} \partial_{z^{\prime}}^{2} \Phi
$$

Assume that $\Phi$ is smooth enough, we introduce its approximation $\Phi_{i}^{n} \approx \Phi\left(z_{i}^{\prime}, t_{n}\right)$. Expanding $\Phi$ in a Taylor series we approximate the following:

$$
\begin{aligned}
\Phi_{i}^{n+1} & =\Phi_{i}^{n}+\sum_{m=1}^{\infty}\left(\partial_{t}^{m} \Phi\right)_{i}^{n} \frac{\tau^{m}}{m !}, \\
\Phi_{i-1}^{n} & =\Phi_{i}^{n}+\sum_{m=1}^{\infty}\left(\partial_{z^{\prime}}^{m} \Phi\right)_{i}^{n} \frac{(-1)^{m} h^{m}}{m !} \\
\Phi_{i+1}^{n} & =\Phi_{i}^{n}+\sum_{m=1}^{\infty}\left(\partial_{z^{\prime}}^{m} \Phi\right)_{i}^{n} \frac{h^{m}}{m !} .
\end{aligned}
$$

Now, we can compute the desired combinations:

$$
\begin{aligned}
2 \Phi_{i}^{n+1}-\Phi_{i+1}^{n}-\Phi_{i-1}^{n}= & 2 \tau\left(\left(\partial_{t} \Phi\right)_{i}^{n}+\sum_{m=1}^{\infty}\left(\partial_{t}^{2 m+1} \Phi\right)_{i}^{n} \frac{\tau^{2 m}}{(2 m+1) !}\right)+ \\
& +2 \sum_{m=1}^{\infty} \frac{1}{(2 m) !}\left(\left(\partial_{t}^{2 m} \Phi\right)_{i}^{n} \tau^{2 m}-\left(\partial_{z^{\prime}}^{2 m} \Phi\right)_{i}^{n} h^{2 m}\right) \\
\Phi_{i+1}^{n}-\Phi_{i-1}^{n}= & 2 h\left(\left(\partial_{z^{\prime}} \Phi\right)_{i}^{n}+\sum_{m=1}^{\infty}\left(\partial_{z^{\prime}}^{2 m+1} \Phi\right)_{i}^{n} \frac{h^{2 m}}{(2 m+1) !}\right) .
\end{aligned}
$$

Thus, according to (3.12) and (3.15) (recall, $N-1 \geq i \geq 1$ ), we get

$$
\begin{aligned}
& \left(\mathcal{P}_{\tau, h} \Phi-\mathcal{P} \Phi\right)_{i}^{n}=\sum_{m=1}^{\infty} \frac{\tau^{2 m-1}}{(2 m) !}\left[\left(\partial_{t}^{2 m} \Phi\right)_{i}^{n}-\left(\frac{h}{\tau}\right)^{2 m}\left(\partial_{z^{\prime}}^{2 m} \Phi\right)_{i}^{n}\right]+ \\
& +\sum_{m=1}^{\infty} \frac{\tau^{2 m}}{(2 m+1) !}\left[\mathbf{I}_{4}\left(\partial_{t}^{2 m+1} \Phi\right)_{i}^{n}+c \mathbf{J}_{4}\left(\frac{h}{\tau}\right)^{2 m}\left(\partial_{z^{\prime}}^{2 m+1} \Phi\right)_{i}^{n}\right]
\end{aligned}
$$


Notice that for further reducing that if we differentiate (3.16) in time twice we get $\partial_{t}^{4} \Phi=c^{2} \partial_{z^{\prime} z^{\prime} t t} \Phi$ and similarly in space: $\partial_{t t z^{\prime} z^{\prime}} \Phi=c^{2} \partial_{z^{\prime}}^{4} \Phi$, hence $\partial_{t}^{4} \Phi=c^{4} \partial_{z^{\prime}}^{4} \Phi$. Using this line of reasoning, we come by induction to

$$
\partial_{t}^{2 m} \Phi=c^{2 m} \partial_{z^{\prime}}^{2 m} \Phi
$$

Now we rewrite $(3.20)$ as

$$
\begin{aligned}
& \left(\mathcal{P}_{\tau, h} \Phi-\mathcal{P} \Phi\right)_{i}^{n}=\sum_{m=1}^{\infty} \frac{\tau^{2 m-1}}{(2 m) !}\left[1-\left(\frac{h}{c \tau}\right)^{2 m}\right]\left(\partial_{t}^{2 m} \Phi\right)_{i}^{n}+ \\
& +\sum_{m=1}^{\infty} \frac{\tau^{2 m}}{(2 m+1) !}\left(\partial_{t}^{2 m}\left[\mathbf{I}_{4} \partial_{t} \Phi+c \mathbf{J}_{4}\left(\frac{h}{c \tau}\right)^{2 m} \partial_{z^{\prime}} \Phi\right]\right)_{i}^{n}
\end{aligned}
$$

Using (3.15), we can conclude that in case $h=c \tau$ both sums in (3.22) vanish. Therefore, in that case, the discretization (3.12) of the operator $\mathcal{P}$ is highly accurate with $p, q>2$. However, if $h \neq c \tau$, the truncation error is $\mathcal{O}\left(\tau+h^{2} / \tau\right)$. More precisely, in the case $h>c \tau$ we add to the scheme artificial diffusion, otherwise, if $h<c \tau$ we create antidiffusion $[101,113]$.

Proposition $3.2\left(\ell^{2}\right.$-stability of Numerical Scheme I). $\ell^{2}$-stability for Scheme I (3.8) holds under the Courant-Fredrichs-Lewy (CFL)-condition: $\frac{c \tau}{h} \leq 1$.

Proof. We explore the $\ell^{2}$-stability of the FDS (3.8) employing von Neumann analysis $[101,113]$. Precisely, we would like to prove, that $\exists C>0$, such that $\left\|\mathbf{U}^{n}\right\|_{h} \leq$ $C\left\|\mathbf{U}^{0}\right\|_{h}, \forall n \leq n_{\max }, \mathbf{U}^{n}=\left(\mathbf{U}_{j}^{n}\right)_{j \in \mathbb{Z}}$, where the grid dependent $\ell^{2}$-norm of the 4dimensional vector $\mathbf{U}^{n}, n \in\left[0, n_{\max }\right]$, is defined by

$$
\left\|\mathbf{U}^{n}\right\|_{h, D}^{2}=\sum_{s=1}^{4} h \sum_{i=1}^{N}\left(U_{s, i}^{n}\right)^{2},
$$

where $U_{1, i}^{n}:=E_{x^{\prime}, i}^{n}, i=0, \ldots, N, n \in\left[0, n_{\max }\right]$, and so on for $s=2,3,4$.

We apply the discrete Fourier transform, $\widehat{\mathbf{U}}^{n}(\xi)=h \sum_{j=-\infty}^{\infty} e^{\mathrm{i} j h \xi} \mathbf{U}_{j}^{n}$, where 
$\xi \in\left(-\frac{\pi}{h}, \frac{\pi}{h}\right)$, to the homogeneous equation $(3.12)\left(\mathbf{F}_{\tau, h}=0\right)$ and using the shift theorem with substitution $\theta=h \xi$, we get

$$
\frac{1}{2 \tau} \mathbf{I}_{4}\left(2 \widehat{\mathbf{U}}^{n+1}(\xi)-\widehat{\mathbf{U}}^{n}(\xi) e^{\mathbf{i} \theta}-\widehat{\mathbf{U}}^{n}(\xi) e^{-\mathbf{i} \theta}\right)+\frac{c}{2 h} \mathbf{J}_{4}\left(\widehat{\mathbf{U}}^{n}(\xi) e^{\mathbf{i} \theta}-\widehat{\mathbf{U}}^{n}(\xi) e^{-\mathbf{i} \theta}\right)=\mathbf{0}
$$

From here, we express $\widehat{\mathbf{U}}^{n+1}(\xi)$ as

$$
\widehat{\mathbf{U}}^{n+1}(\xi)=\left(\mathbf{I}_{4} \frac{e^{\mathrm{i} \theta}+e^{-\mathbf{i} \theta}}{2}-\frac{c \tau}{h} \mathbf{J}_{4} \frac{e^{\mathrm{i} \theta}-e^{-\mathbf{i} \theta}}{2}\right) \widehat{\mathbf{U}}^{n}(\xi)=\left(\mathbf{I}_{4} \cos \theta-i \frac{c \tau}{h} \mathbf{J}_{4} \sin \theta\right) \widehat{\mathbf{U}}^{n}(\xi) .
$$

Let us introduce $L^{2}$-norm of $\widehat{\mathbf{U}}^{n}(\xi)$ as

$$
\left\|\widehat{\mathbf{U}}^{n}\right\|_{\left(L^{2}(-\pi / h, \pi / h)\right)}^{2}=\frac{1}{2 \pi} \sum_{s=1}^{4} \int_{-\pi / h}^{\pi / h}\left|\widehat{U}_{s}^{n}(\xi)\right|^{2} d \xi
$$

According to Parseval's equality we have $\left\|\mathbf{U}^{n}\right\|_{h}^{2}=\left\|\widehat{\mathbf{U}}^{n}\right\|_{\left(L^{2}(-\pi / h, \pi / h)\right.}^{2}{ }^{4}$. Now we compute the eigenvalues, $Z_{1, \ldots, 4}$, of the amplification matrix $G(\theta):=\mathbf{I}_{4} \cos \theta-i \frac{c \tau}{h} \mathbf{J}_{4} \sin \theta$, see (3.25), which are

$$
Z_{1,2}=\cos \theta \pm i \frac{c \tau}{h} \sin \theta, \quad \text { and } \quad Z_{3}=Z_{1}, \quad Z_{4}=Z_{2}
$$

To obtain the condition of stability, we set $\left|Z_{i}\right| \leq 1, i=1, \ldots, 4$, which gives

$$
\left|Z_{i}\right|^{2}=\cos ^{2} \theta+\left(\frac{c \tau}{h}\right)^{2} \sin ^{2} \theta=1-\sin ^{2} \theta\left(1-\left(\frac{c \tau}{h}\right)^{2}\right) \leq 1
$$

Therefore, we can expect the $\ell^{2}$-stability for the Lax-Friedrichs scheme holds under the CFL-condition: $\frac{c \tau}{h} \leq 1[49,101]$.

Combining the stability condition with the condition for the best scheme accuracy, which was obtained as Proposition 3.1, one gets $\frac{c \tau}{h}=1$. In practice, see Section 4.1, to meet the stability condition and to reduce the artificial diffusion, the CFL-number 
$\alpha:=\frac{c \tau}{h}$ was set equal to 0.99 . Thus, we deal with the first order scheme.

Finally, to make a conclusion about convergence of the numerical scheme (3.8) proposed for MEs we use,

Theorem 3.1 (Lax-Richtmyer Equivalence theorem). A consistent one-step FDS for a well-posed initial-value problem for a PDE is convergent if and only if it is stable.

Proof. See, e.g. [113].

Thus, keeping in mind Propositions 3.1 and 3.2, and following Lax-Richtmyer theorem, we state that (3.8) is convergent under CFL-conditions.

\subsubsection{Numerical Scheme II}

We now incorporate the polarization evolution equation introduced in Section 2.3 into the MASP model, in the case of LP-pulse. With this purpose we take only the second and third equations of the system (3.3):

$$
\left\{\begin{array}{l}
\partial_{t} E_{y^{\prime}}=c \partial_{z^{\prime}} B_{x^{\prime}}-4 \pi \partial_{t} P_{y^{\prime}} \\
\partial_{t} B_{x^{\prime}}=c \partial_{z^{\prime}} E_{y^{\prime}}
\end{array}\right.
$$

As before we introduce the vector function $\mathbf{U}: \mathbb{R} \times \mathbb{R}_{+} \rightarrow \mathbb{R}^{2}$, which in this instance reads

$$
\mathbf{U}\left(z^{\prime}, t\right)=\left(E_{y^{\prime}}\left(z^{\prime}, t\right), B_{x^{\prime}}\left(z^{\prime}, t\right)\right)^{T},
$$

so that on the same as in section 3.1 .1 grid $\mathbf{U}_{i}^{n} \approx \mathbf{U}\left(z_{i}^{\prime}, t_{n}\right)$. We can then rewrite the system (3.28) in the form of nonhomogeneous one-direction wave equation:

$$
\partial_{t} \mathbf{U}=c \mathbf{J}_{2} \partial_{z^{\prime}} \mathbf{U}+\mathbf{F},
$$


where $\mathbf{J}_{2}=\left[\begin{array}{ll}0 & 1 \\ 1 & 0\end{array}\right]$ and $\mathbf{F}\left(z^{\prime}, t\right)=\left(-4 \pi \partial_{t} P_{y^{\prime}}\left(z^{\prime}, t\right), 0\right)^{T}$.

We need a more accurate scheme than (3.8), at least of second-order accuracy in time and space. With this aim we choose the Lax-Wendroff-like method. Following the procedure described in [113], we write down the Taylor expansion:

$$
\mathbf{U}\left(z^{\prime}, t+\tau\right)=\mathbf{U}\left(z^{\prime}, t\right)+\tau \partial_{t} \mathbf{U}\left(z^{\prime}, t\right)+\frac{\tau^{2}}{2} \partial_{t}^{2} \mathbf{U}\left(z^{\prime}, t\right)+\mathcal{O}\left(\tau^{3}\right)
$$

To replace the partial derivatives in time by spatial derivatives we use the equation (3.30) itself for the first derivative, and differentiate it in time to obtain the second derivative (notice that $\mathbf{J}_{2}^{2}=\mathbf{I}_{2}$ ):

$$
\partial_{t}^{2} \mathbf{U}=c \mathbf{J}_{2} \partial_{z t} \mathbf{U}+\partial_{t} \mathbf{F}=c^{2} \partial_{z^{\prime}}^{2} \mathbf{U}+c \mathbf{J}_{2} \partial_{z^{\prime}} \mathbf{F}+\partial_{t} \mathbf{F}
$$

Thus

$$
\begin{aligned}
\mathbf{U}\left(z^{\prime}, t+\tau\right)=\mathbf{U}\left(z^{\prime}, t\right)+\tau c \mathbf{J}_{2} \partial_{z^{\prime}} \mathbf{U}\left(z^{\prime}, t\right)+\tau \mathbf{F}+ \\
\quad+\frac{\tau^{2}}{2}\left(c^{2} \partial_{z^{\prime}}^{2} \mathbf{U}\left(z^{\prime}, t\right)+c \mathbf{J}_{2} \partial_{z^{\prime}} \mathbf{F}+\partial_{t} \mathbf{F}\right)+\mathcal{O}\left(\tau^{3}\right) .
\end{aligned}
$$

Approximating the space derivatives of $\mathbf{U}$ by second-order accurate differences:

$$
\begin{aligned}
\left(\partial_{z^{\prime}} \mathbf{U}\right)_{i}^{n} & =\frac{\mathbf{U}_{i+1}^{n}-\mathbf{U}_{i-1}^{n}}{2 h}+\mathcal{O}\left(h^{2}\right), \\
\left(\partial_{z^{\prime}}^{2} \mathbf{U}\right)_{i}^{n} & =\frac{\mathbf{U}_{i+1}^{n}-2 \mathbf{U}_{i}^{n}+\mathbf{U}_{i-1}^{n}}{h^{2}}+\mathcal{O}\left(h^{2}\right),
\end{aligned}
$$

and employing for derivatives of $\mathbf{F}$ in time and space:

$$
\begin{aligned}
\left(\partial_{t} \mathbf{F}\right)_{i}^{n} & =\frac{\mathbf{F}_{i}^{n+1}-\mathbf{F}_{i}^{n}}{\tau}, \\
\left(\partial_{z^{\prime}} \mathbf{F}\right)_{i}^{n} & =\frac{\mathbf{F}_{i+1}^{n}-\mathbf{F}_{i-1}^{n}}{2 h},
\end{aligned}
$$

we come to the Lax-Wendroff scheme, which in our case is

$$
\begin{array}{r}
\mathbf{U}_{i}^{n+1}=\mathbf{U}_{i}^{n}+\frac{\alpha}{2}\left[\mathbf{J}_{2}\left(\mathbf{U}_{i+1}^{n}-\mathbf{U}_{i-1}^{n}\right)+\alpha\left(\mathbf{U}_{i+1}^{n}-2 \mathbf{U}_{i}^{n}+\mathbf{U}_{i-1}^{n}\right)\right]+ \\
+\frac{\mathbf{F}_{i}^{n+1}+\mathbf{F}_{i}^{n}}{2} \tau+\frac{\alpha}{4} \mathbf{J}_{2}\left(\mathbf{F}_{i+1}^{n}-\mathbf{F}_{i-1}^{n}\right) \tau
\end{array}
$$


where, as well as in Section 3.1.1, $\alpha=\frac{c \tau}{h}$ denotes the CFL-number.

Finally, the scheme in terms of the electric, $E:=E_{y^{\prime}}$, and magnetic, $B:=B_{x^{\prime}}$, fields writes as:

$$
\left\{\begin{aligned}
& E_{i}^{n+1}=E_{i}^{n}+\frac{\alpha}{2}\left[B_{i+1}^{n}-B_{i-1}^{n}+\right.\left.\alpha\left(E_{i+1}^{n}-2 E_{i}^{n}+B_{i-1}^{n}\right)\right]- \\
&-2 \pi\left[\left(\partial_{t} P_{y^{\prime}}\right)_{i}^{n+1}+\left(\partial_{t} P_{y^{\prime}}\right)_{i}^{n}\right] \tau \\
& B_{i}^{n+1}=B_{i}^{n}+\frac{\alpha}{2}\left[E_{i+1}^{n}-E_{i-1}^{n}+\alpha\right.\left.\left(B_{i+1}^{n}-2 B_{i}^{n}+B_{i-1}^{n}\right)\right]- \\
&-\alpha \pi\left[\left(\partial_{t} P_{y^{\prime}}\right)_{i+1}^{n}-\left(\partial_{t} P_{y^{\prime}}\right)_{i-1}^{n}\right] \tau
\end{aligned}\right.
$$

Proposition 3.3 $\left(\ell^{2}\right.$-stability of Numerical Scheme II). Scheme (3.32) is $\ell^{2}$ stable under CFL-condition: $\frac{c \tau}{h} \leq 1$.

Proof. We set $\mathbf{F}=\mathbf{0}$ in (3.32) and then apply von Neumann stability analysis the same way as it was done in Proposition 3.2. Thus, we write

$$
\widehat{\mathbf{U}}^{n+1}(\xi)=\left[\mathbf{I}_{2}\left(1-\alpha^{2}+\alpha^{2} \cos \theta\right)+i \alpha \mathbf{J}_{2} \sin \theta\right] \widehat{\mathbf{U}}^{n}(\xi) \equiv G(\theta) \widehat{\mathbf{U}}^{n}(\xi)
$$

where $\alpha=\frac{c \tau}{h}$, and $G(\theta)$ denotes the amplification matrix. The eigenvalues of this matrix are complex conjugate:

$$
Z_{1,2}(\theta)=1-2 \alpha^{2} \sin ^{2} \frac{\theta}{2} \pm i \alpha \sin \theta
$$

and their amplitudes:

$$
\left|Z_{1,2}(\theta)\right|^{2}=1-4 \alpha^{2}\left(1-\alpha^{2}\right) \sin ^{4} \frac{\theta}{2}
$$

From here one gets the same condition of stability as above in the case of the LaxFriedrichs scheme: $\frac{c \tau}{h} \leq 1$, see Proposition 3.2.

Thus, with Proposition 3.3 and Theorem 3.1 we confirm convergence of the secondorder consistent Scheme (3.33). 


\subsubsection{Propagating of the LP-pulse: Perturbative Approach}

In this section we present a numerical scheme for modeling 1d Maxwell's equations in the perturbative regime. Further, in Section 4 we will use this simple model as reference one.

We consider the equation (3.28) with perturbative expression for the polarization $[24]:$

$$
P_{y^{\prime}}=\chi^{(1)} E_{y^{\prime}}+\chi^{(3)} E_{y^{\prime}}^{3}
$$

namely

$$
\left\{\begin{array}{l}
\partial_{t} E_{y^{\prime}}=c \partial_{z^{\prime}} B_{x^{\prime}}-4 \pi \chi^{(1)} \partial_{t} E_{y^{\prime}}-12 \pi \chi^{(3)} E_{y^{\prime}}^{2} \partial_{t} E_{y^{\prime}} \\
\partial_{t} B_{x^{\prime}}=c \partial_{z^{\prime}} E_{y^{\prime}}
\end{array}\right.
$$

Where $\chi^{(1)}$ and $\chi^{(3)}$ are coefficients of instantaneous susceptibilities: linear and cubicnonlinear, respectively. Introducing the variable function

$$
a\left(E_{y^{\prime}}\right):=\left(1+4 \pi \chi^{(1)}+12 \pi \chi^{(3)} E_{y^{\prime}}^{2}\right)^{-1}
$$

we rewrite to the system as

$$
\left\{\begin{array}{l}
\partial_{t} E_{y^{\prime}}=c a\left(E_{y^{\prime}}\right) \partial_{z^{\prime}}\left(B_{x^{\prime}}\right) \\
\partial_{t} B_{x^{\prime}}=c \partial_{z^{\prime}} E_{y^{\prime}}
\end{array}\right.
$$

To obtain the second order accuracy in time we write the solution of (3.38) in the form:

$$
\begin{aligned}
& E_{y^{\prime}}\left(z^{\prime}, t+\tau\right)=E_{y^{\prime}}\left(z^{\prime}, t\right)+\tau \partial_{t} E_{y^{\prime}}\left(z^{\prime}, t\right)+\frac{\tau^{2}}{2} \partial_{t}^{2} E_{y^{\prime}}\left(z^{\prime}, t\right)+\mathcal{O}\left(\tau^{3}\right), \\
& B_{x^{\prime}}\left(z^{\prime}, t+\tau\right)=B_{x^{\prime}}\left(z^{\prime}, t\right)+\tau \partial_{t} B_{x^{\prime}}\left(z^{\prime}, t\right)+\frac{\tau^{2}}{2} \partial_{t}^{2} B_{x^{\prime}}\left(z^{\prime}, t\right)+\mathcal{O}\left(\tau^{3}\right) .
\end{aligned}
$$


With the first temporal derivatives from the system (3.38), we can express the second derivatives in time through the partial derivatives in space:

$$
\begin{aligned}
& \partial_{t}^{2} E_{y^{\prime}}=c^{2} a\left(E_{y^{\prime}}\right) \partial_{z^{\prime}}^{2} E_{y^{\prime}}-c^{2} b\left(E_{y^{\prime}}\right) a^{3}\left(E_{y^{\prime}}\right)\left(\partial_{z^{\prime}} B_{x^{\prime}}\right)^{2}, \\
& \partial_{t}^{2} B_{x^{\prime}}=c^{2} \partial_{z^{\prime}} a\left(E_{y^{\prime}}\right) \partial_{z^{\prime}} B_{x^{\prime}}
\end{aligned}
$$

where

$$
b\left(E_{y^{\prime}}\right):=24 \pi \chi^{(3)} E_{y^{\prime}} .
$$

Now, using the second order accurate approximation for the spatial derivatives, we obtain the Lax-Wendroff scheme for $E:=E_{y^{\prime}}$ and $B:=B_{x^{\prime}}$ :

$$
\left\{\begin{array}{c}
E_{i}^{n+1}=E_{i}^{n}+\frac{\alpha}{2} a_{i}^{n}\left(B_{i+1}^{n}-B_{i-1}^{n}\right)+\frac{\alpha^{2}}{2} a_{i}^{n}\left(E_{i+1}^{n}-2 E_{i}^{n}+E_{i-1}^{n}\right)- \\
-\frac{\alpha^{2}}{8}\left(a_{i}^{n}\right)^{3} b_{i}^{n}\left(B_{i+1}^{n}-B_{i-1}^{n}\right)^{2} \\
B_{i}^{n+1}=B_{i}^{n}+\frac{\alpha}{2}\left(E_{i+1}^{n}-E_{i-1}^{n}\right)+\frac{\alpha^{2}}{2}\left[a_{i+\frac{1}{2}}^{n}\left(B_{i+1}^{n}-B_{i}^{n}\right)-\right. \\
\left.-a_{i-\frac{1}{2}}^{n}\left(B_{i}^{n}-B_{i-1}^{n}\right)\right]
\end{array}\right.
$$

where coefficients $a_{i \pm \frac{1}{2}}^{n}$ are evaluated at $\frac{E_{i}^{n}+E_{i \pm 1}^{n}}{2}$.

Finally, we need to provide some comments about the convergence of the scheme under consideration. Since Scheme (3.41) contains variable coefficients $a_{i}^{n}$ and $b_{i}^{n}$, moreover, it is nonlinear with respect to the finite difference $B_{i+1}^{n}-B_{i-1}^{n}$, the stability condition for it cannot be obtained analytically as it was done in Proposition 3.3 for the scheme with constant coefficients. Sometimes, one can rely on the "CFL-like" condition $\left|a_{i}^{n}\right| c \tau / h \leq 1$, see [113], however, because of the nonlinear term it seems doubtful as well. We return to the question about stability of (3.41) in Section 4.2.1, where we present the computational data. 


\subsection{Discretization of 3d Maxwell's Equations}

We now consider the components of the EM-field, in the general form

$$
\left\{\begin{array}{l}
\mathbf{E}\left(\mathbf{x}^{\prime}, t\right)=\left(E_{x^{\prime}}\left(x^{\prime}, y^{\prime}, z^{\prime}, t\right), E_{y^{\prime}}\left(x^{\prime}, y^{\prime}, z^{\prime}, t\right), E_{z^{\prime}}\left(x^{\prime}, y^{\prime}, z^{\prime}, t\right)\right)^{T} \\
\mathbf{B}\left(\mathbf{x}^{\prime}, t\right)=\left(B_{x^{\prime}}\left(x^{\prime}, y^{\prime}, z^{\prime}, t\right), B_{y^{\prime}}\left(x^{\prime}, y^{\prime}, z^{\prime}, t\right), B_{z^{\prime}}\left(x^{\prime}, y^{\prime}, z^{\prime}, t\right)\right)^{T}
\end{array}\right.
$$

Then the MEs (3.2) in a cartesian coordinate system read

$$
\left\{\begin{array}{l}
\partial_{t} E_{x^{\prime}}=c\left(\partial_{y^{\prime}} B_{z^{\prime}}-\partial_{z^{\prime}} B_{y^{\prime}}\right)-4 \pi\left(\partial_{t} P_{x^{\prime}}+J_{x^{\prime}}\right) \\
\partial_{t} E_{y^{\prime}}=c\left(\partial_{z^{\prime}} B_{x^{\prime}}-\partial_{x^{\prime}} B_{z^{\prime}}\right)-4 \pi\left(\partial_{t} P_{y^{\prime}}+J_{y^{\prime}}\right) \\
\partial_{t} E_{z^{\prime}}=c\left(\partial_{x^{\prime}} B_{y^{\prime}}-\partial_{y^{\prime}} B_{x^{\prime}}\right)-4 \pi\left(\partial_{t} P_{z^{\prime}}+J_{z^{\prime}}\right) \\
\partial_{t} B_{x^{\prime}}=c\left(\partial_{z^{\prime}} E_{y^{\prime}}-\partial_{y^{\prime}} E_{z^{\prime}}\right) \\
\partial_{t} B_{y^{\prime}}=c\left(\partial_{x^{\prime}} E_{z^{\prime}}-\partial_{z^{\prime}} E_{x^{\prime}}\right) \\
\partial_{t} B_{z^{\prime}}=c\left(\partial_{y^{\prime}} E_{x^{\prime}}-\partial_{x^{\prime}} E_{y^{\prime}}\right)
\end{array}\right.
$$

The appropriate BCs for (3.43) will be discussed in Section 3.2.2.

\subsubsection{Yee's Numerical Scheme}

To discretize (3.43), we use a classical scheme proposed by Yee [123], also knows as the Finite-Difference Time Domain (FDTD) method. This discretization uses compact explicit centred differences on a structured staggered grid, see Figure 3.2.

Following [123], let us index spatial grid points as $(k, l, m)(k, l, m \geq 1)$, respectively $\left(k \Delta x^{\prime}, l \Delta y^{\prime}, m \Delta z^{\prime}\right)$, and we approximate the field-vector

$$
\mathbf{U}\left(\mathbf{x}^{\prime}, t\right)=\left(E_{x^{\prime}}\left(\mathbf{x}^{\prime}, t\right), E_{y^{\prime}}\left(\mathbf{x}^{\prime}, t\right), E_{z^{\prime}}\left(\mathbf{x}^{\prime}, t\right), B_{x^{\prime}}\left(\mathbf{x}^{\prime}, t\right), B_{y^{\prime}}\left(\mathbf{x}^{\prime}, t\right), B_{z^{\prime}}\left(\mathbf{x}^{\prime}, t\right)\right)^{T}
$$

by $\mathbf{U}^{n}(k, l, m) \approx \mathbf{U}\left(k \Delta x^{\prime}, l \Delta y^{\prime}, m \Delta z^{\prime}, n \tau\right)$. 


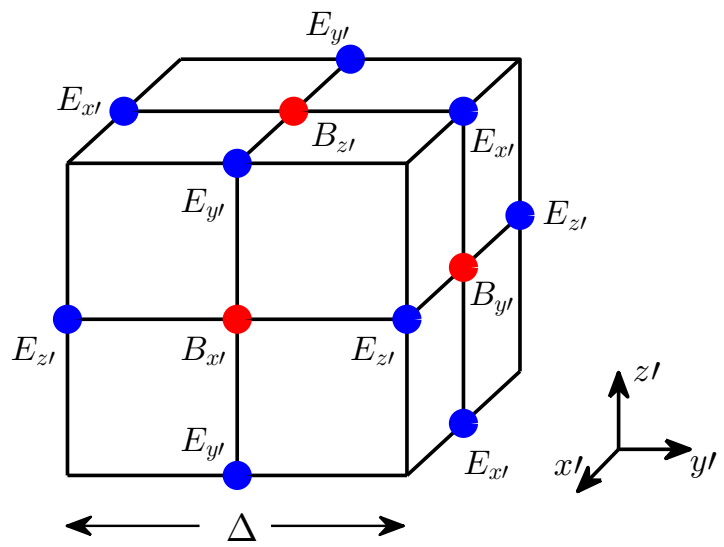

Figure 3.2: Staggered grid for Yee's scheme

The first three equations of the system (3.43) are discretize as follow

$$
\begin{gathered}
\frac{E_{x^{\prime}}^{n}\left(k+\frac{1}{2}, l, m\right)-E_{x^{\prime}}^{n-1}\left(k+\frac{1}{2}, l, m\right)}{\tau}= \\
=c \frac{B_{z^{\prime}}^{n-\frac{1}{2}}\left(k+\frac{1}{2}, l+\frac{1}{2}, m\right)-B_{z^{\prime}}^{n-\frac{1}{2}}\left(k+\frac{1}{2}, l-\frac{1}{2}, m\right)}{\Delta y^{\prime}}- \\
-c \frac{B_{y^{\prime}}^{n-\frac{1}{2}}\left(k+\frac{1}{2}, l, m+\frac{1}{2}\right)-B_{y^{\prime}}^{n-\frac{1}{2}}\left(k+\frac{1}{2}, l, m-\frac{1}{2}\right)}{\Delta z^{\prime}}- \\
\frac{-4 \pi\left(\partial_{t} P_{x^{\prime}}{ }^{n-\frac{1}{2}}\left(k+\frac{1}{2}, l, m\right)+J_{x^{\prime}}^{n-\frac{1}{2}}\left(k+\frac{1}{2}, l, m\right)\right),}{E_{y^{\prime}}^{n}\left(k, l+\frac{1}{2}, m\right)-E_{y^{\prime}}^{n-1}\left(k, l+\frac{1}{2}, m\right)} \\
\tau \\
=c \frac{B_{x^{\prime}}^{n-\frac{1}{2}}\left(k, l+\frac{1}{2}, m+\frac{1}{2}\right)-B_{x^{\prime}}^{n-\frac{1}{2}}\left(k, l+\frac{1}{2}, m-\frac{1}{2}\right)}{\Delta z^{\prime}}- \\
-c \frac{B_{z^{\prime}}^{n-\frac{1}{2}}\left(k+\frac{1}{2}, l+\frac{1}{2}, m\right)-B_{z^{\prime}}^{n-\frac{1}{2}}\left(k-\frac{1}{2}, l+\frac{1}{2}, m\right)}{\Delta x^{\prime}}- \\
-4 \pi\left(\partial_{t} P_{y^{\prime}}^{n-\frac{1}{2}}\left(k, l+\frac{1}{2}, m\right)+J_{y^{\prime}}^{n-\frac{1}{2}}\left(k, l+\frac{1}{2}, m\right)\right),
\end{gathered}
$$

$$
\begin{aligned}
& \frac{E_{z^{\prime}}^{n}\left(k, l, m+\frac{1}{2}\right)-E_{z^{\prime}}^{n-1}\left(k, l, m+\frac{1}{2}\right)}{\tau}= \\
& =c \frac{B_{y^{\prime}}^{n-\frac{1}{2}}\left(k+\frac{1}{2}, l, m+\frac{1}{2}\right)-B_{y^{\prime}}^{n-\frac{1}{2}}\left(k-\frac{1}{2}, l, m+\frac{1}{2}\right)}{\Delta x^{\prime}}- \\
& \quad-c \frac{B_{x^{\prime}}^{n-\frac{1}{2}}\left(k, l+\frac{1}{2}, m+\frac{1}{2}\right)-B_{x^{\prime}}^{n-\frac{1}{2}}\left(k, l-\frac{1}{2}, m+\frac{1}{2}\right)}{\Delta y^{\prime}}- \\
& \quad-4 \pi\left(\partial_{t} P_{z^{\prime}}{ }^{n-\frac{1}{2}}\left(k, l, m+\frac{1}{2}\right)+J_{z^{\prime}}^{n-1 / 2}\left(k, l, m+\frac{1}{2}\right)\right) .
\end{aligned}
$$


The last three equations of (3.43) on the staggered grid:

$$
\begin{gathered}
\frac{B_{x^{\prime}}^{n+\frac{1}{2}}\left(k, l+\frac{1}{2}, m+\frac{1}{2}\right)-B_{x^{\prime}}^{n-\frac{1}{2}}\left(k, l+\frac{1}{2}, m+\frac{1}{2}\right)}{\tau}= \\
=c \frac{E_{y^{\prime}}^{n}\left(k, l+\frac{1}{2}, m+1\right)-E_{y^{\prime}}^{n}\left(k, l+\frac{1}{2}, m\right)}{\Delta z^{\prime}}- \\
-c \frac{E_{z^{\prime}}^{n}\left(k, l+1, m+\frac{1}{2}\right)-E_{z^{\prime}}^{n}\left(k, l, m+\frac{1}{2}\right)}{\Delta y^{\prime}}, \\
\frac{B_{y^{\prime}}^{n+\frac{1}{2}}\left(k+\frac{1}{2}, l, m+\frac{1}{2}\right)-B_{y^{\prime}}^{n-\frac{1}{2}}\left(k+\frac{1}{2}, l, m+\frac{1}{2}\right)}{\tau}= \\
=c \frac{E_{z^{\prime}}^{n}\left(k+1, l, m+\frac{1}{2}\right)-E_{z^{\prime}}^{n}\left(k, l, m+\frac{1}{2}\right)}{\Delta x^{\prime}}- \\
-c \frac{E_{x^{\prime}}^{n}\left(k+\frac{1}{2}, l, m+1\right)-E_{x^{\prime}}^{n}\left(k+\frac{1}{2}, l, m\right)}{\Delta z^{\prime}} \\
\frac{B_{z^{\prime}}^{n+\frac{1}{2}}\left(k+\frac{1}{2}, l+\frac{1}{2}, m\right)-B_{z^{\prime}}^{n-\frac{1}{2}}\left(k+\frac{1}{2}, l+\frac{1}{2}, m\right)}{\tau}= \\
=c \frac{E_{x^{\prime}}^{n}\left(k+\frac{1}{2}, l+1, m\right)-E_{x^{\prime}}^{n}\left(k+\frac{1}{2}, l, m\right)}{\Delta y^{\prime}}- \\
-c \frac{E_{y^{\prime}}^{n}\left(k+1, l+\frac{1}{2}, m\right)-E_{y^{\prime}}^{n}\left(k, l+\frac{1}{2}, m\right)}{\Delta x^{\prime}}
\end{gathered} .
$$

In vacuum, when $P_{x^{\prime}}, P_{y^{\prime}}, J_{x^{\prime}}, J_{y^{\prime}}, J_{z^{\prime}}$ are zeros, the Yee scheme leads to

$$
\begin{aligned}
& \frac{E_{x^{\prime}}^{n}\left(k+\frac{1}{2}, l, m\right)-E_{x^{\prime}}^{n}\left(k-\frac{1}{2}, l, m\right)}{\Delta x^{\prime}}+\frac{E_{y^{\prime}}^{n}\left(k, l+\frac{1}{2}, m\right)-E_{y^{\prime}}^{n}\left(k, l-\frac{1}{2}, m\right)}{\Delta y^{\prime}}+ \\
& +\frac{E_{z^{\prime}}^{n}\left(k, l, m+\frac{1}{2}\right)-E_{z^{\prime}}^{n}\left(k, l, m-\frac{1}{2}\right)}{\Delta z^{\prime}}=\frac{E_{x^{\prime}}^{n-1}\left(k+\frac{1}{2}, l, m\right)-E_{x^{\prime}}^{n-1}\left(k-\frac{1}{2}, l, m\right)}{\Delta x^{\prime}}+ \\
& +\frac{E_{y^{\prime}}^{n-1}\left(k, l+\frac{1}{2}, m\right)-E_{y^{\prime}}^{n-1}\left(k, l-\frac{1}{2}, m\right)}{\Delta y^{\prime}}+\frac{E_{z^{\prime}}^{n-1}\left(k, l, m+\frac{1}{2}\right)-E_{z^{\prime}}^{n-1}\left(k, l, m-\frac{1}{2}\right)}{\Delta z^{\prime}},
\end{aligned}
$$

or shorter, in the symbolic form: $\nabla_{h} \cdot \mathbf{E}_{h}^{n}=\nabla_{h} \cdot \mathbf{E}_{h}^{n+1}$ (here $h=\left(\Delta x^{\prime}, \Delta y^{\prime}, \Delta z^{\prime}\right)$ ), which is consistent with Maxwell-Gauss' equation $\nabla \cdot \mathbf{E}=0$ if $\nabla_{h} \cdot \mathbf{E}_{h}^{0}=0$. In the case of polarizable medium, it can be shown that the Yee scheme is consistent with $\nabla \cdot \mathbf{D}=0$, the condition of plasma quasineutrality, or in FDS terms, $\nabla_{h} \cdot \mathbf{D}_{h}^{n}=0$ for all $n$, provided that $\nabla_{h} \cdot \mathbf{D}_{h}^{0}=0,[79]$. 


\subsubsection{Mur's Absorbing Boundary Conditions}

Further, we should limit the numerical domain, as it cannot be "infinite" in computations. Since now we deal with 3d equations, with the goal to reduce computational cost, it would be desirable to reduce the domain size to that extend as the physical conditions allow. To this end, we use Mur's second order absorbing boundary conditions (ABCs) [91]. In fact, the Yee's scheme requires the ABCs for the three components of the electric field only. Indeed, for the mesh in Fig. 3.2, the B-components are normal to the faces of the represented cube, while the $\mathbf{E}$-components are tangent to its edges. Thus, according to (3.45) to compute the vector $\mathbf{E}$ on the cube, we have to know the value of the $\mathbf{B}$-components outside of the mesh, while to compute $\mathbf{B}$ on the boundary we need to know the value of the E-component on the mesh boundary, see (3.46).

To derive the $\mathrm{BCs}$, we use the fact that each of the electric field components $\mathbf{E}\left(\mathbf{x}^{\prime}, t\right)$ satisfies the $3 \mathrm{~d}$ wave equations in vacuum:

$$
\left[\partial_{t}^{2}-c^{2}\left(\partial_{x^{\prime}}^{2}+\partial_{y^{\prime}}^{2}+\partial_{z^{\prime}}^{2}\right)\right] \mathbf{E}\left(\mathbf{x}^{\prime}, t\right)=\mathbf{0}
$$

To derive the ABCs, only the outgoing waves should be considered. For definiteness we will consider the boundary conditions for the plane $x^{\prime}=0$, assuming that the mesh is located in the region $x^{\prime} \geq 0$. Then, one can write down the one-way wave equation for a space-time plane-wave $\left(\omega, k_{x^{\prime}}, k_{y^{\prime}}, k_{z^{\prime}}\right)$, e.g. in the case of $E_{y^{\prime}}$ component, as

$$
\left.\left(\partial_{t}-v_{x^{\prime}} \partial_{x^{\prime}}\right) E_{y^{\prime}}\right|_{x^{\prime}=0}=0
$$

where $v_{x^{\prime}}=\omega / k_{x^{\prime}}$ and $k_{x^{\prime}}=\sqrt{(\omega / c)^{2}-k_{y^{\prime}}^{2}-k_{z^{\prime}}^{2}}$. Up to the second order $k_{x^{\prime}}=$ $\frac{\omega}{c}-\frac{1}{2} \frac{c}{\omega}\left(k_{y^{\prime}}^{2}+k_{z^{\prime}}^{2}\right)+\mathcal{O}\left(\left(\frac{c}{\omega}\right)^{3}\left(k_{y^{\prime}}^{2}+k_{z^{\prime}}^{2}\right)^{2}\right)$ hereupon (3.48) is shaped into

$$
\left.\left[\frac{\omega}{c^{2}} \partial_{t}-\frac{1}{2}\left(k_{y^{\prime}}^{2}+k_{z^{\prime}}^{2}\right) \frac{1}{\omega} \partial_{t}-\frac{\omega}{c} \partial_{x^{\prime}}\right] E_{y^{\prime}}\right|_{x^{\prime}=0}=0
$$


In the case of the plane-wave $\partial_{t} E_{y^{\prime}}=-i \omega E_{y^{\prime}}$ and $k_{y^{\prime}}^{2} E_{y^{\prime}}=-\partial_{y^{\prime}}^{2} E_{y^{\prime}}, k_{z^{\prime}}^{2} E_{y^{\prime}}=-\partial_{z^{\prime}}^{2} E_{y^{\prime}}$, so that the desired boundary condition takes the form [91]:

$$
\left.\left[\frac{1}{c} \partial_{t x^{\prime}}-\frac{1}{c^{2}} \partial_{t}^{2}+\frac{1}{2}\left(\partial_{y^{\prime}}^{2}+\partial_{z^{\prime}}^{2}\right)\right] E_{y^{\prime}}\right|_{x^{\prime}=0}=0
$$

Following Mur's BC, we approximate the second order derivatives in the above equation with second order centered differences in both the space and the time, so that for $\left.\partial_{t x^{\prime}} E_{y^{\prime}}\right|_{x^{\prime}=0}$ it reads

$$
\frac{E_{y^{\prime}}^{n+1}\left(1, l+\frac{1}{2}, m\right)-E_{y^{\prime}}^{n-1}\left(1, l+\frac{1}{2}, m\right)-E_{y^{\prime}}^{n+1}\left(0, l+\frac{1}{2}, m\right)+E_{y^{\prime}}^{n-1}\left(0, l+\frac{1}{2}, m\right)}{2 \Delta x^{\prime} \tau}
$$

and then for $\left.\partial_{t}^{2} E_{y^{\prime}}\right|_{x^{\prime}=0}$ :

$$
\begin{array}{r}
\frac{E_{y^{\prime}}^{n+1}\left(0, l+\frac{1}{2}, m\right)-2 E_{y^{\prime}}^{n}\left(0, l+\frac{1}{2}, m\right)+E_{y^{\prime}}^{n-1}\left(0, l+\frac{1}{2}, m\right)}{2 \tau^{2}}+ \\
+\frac{E_{y^{\prime}}^{n+1}\left(1, l+\frac{1}{2}, m\right)-2 E_{y^{\prime}}^{n}\left(1, l+\frac{1}{2}, m\right)+E_{y^{\prime}}^{n-1}\left(1, l+\frac{1}{2}, m\right)}{2 \tau^{2}}
\end{array}
$$

for $\left.\partial_{y^{\prime}}^{2} E_{y^{\prime}}\right|_{x^{\prime}=0}$ :

$$
\begin{aligned}
\frac{E_{y^{\prime}}^{n}\left(0, l+\frac{3}{2}, m\right)-2 E_{y^{\prime}}^{n}\left(0, l+\frac{1}{2}, m\right)+E_{y^{\prime}}^{n}\left(0, l-\frac{1}{2}, m\right)}{2\left(\Delta y^{\prime}\right)^{2}}+ \\
+\frac{E_{y^{\prime}}^{n}\left(1, l+\frac{3}{2}, m\right)-2 E_{y^{\prime}}^{n}\left(1, l+\frac{1}{2}, m\right)+E_{y^{\prime}}^{n}\left(1, l-\frac{1}{2}, m\right)}{2\left(\Delta y^{\prime}\right)^{2}},
\end{aligned}
$$

and for $\left.\partial_{z^{\prime}}^{2} E_{y^{\prime}}\right|_{x^{\prime}=0}$ :

$$
\begin{aligned}
& \frac{E_{y^{\prime}}^{n}\left(0, l+\frac{1}{2}, m+1\right)-2 E_{y^{\prime}}^{n}\left(0, l+\frac{1}{2}, m\right)+E_{y^{\prime}}^{n}\left(0, l+\frac{1}{2}, m-1\right)}{2\left(\Delta z^{\prime}\right)^{2}}+ \\
& \quad+\frac{E_{y^{\prime}}^{n}\left(1, l+\frac{1}{2}, m+1\right)-2 E_{y^{\prime}}^{n}\left(1, l+\frac{1}{2}, m\right)+E_{y^{\prime}}^{n}\left(1, l+\frac{1}{2}, m-1\right)}{2\left(\Delta z^{\prime}\right)^{2}} .
\end{aligned}
$$


Finally, to discretize (3.49), we need to combine (3.50)-(3.53) resulting in:

$$
\begin{aligned}
& E_{y^{\prime}}^{n+1}\left(0, l+\frac{1}{2}, m\right)=-E_{y^{\prime}}^{n-1}\left(1, l+\frac{1}{2}, m\right)+\frac{c \tau-\Delta x^{\prime}}{c \tau+\Delta x^{\prime}}\left[E_{y^{\prime}}^{n+1}\left(1, l+\frac{1}{2}, m\right)+\right. \\
& \left.+E_{y^{\prime}}^{n-1}\left(0, l+\frac{1}{2}, m\right)\right]+\frac{2 \Delta x^{\prime}}{c \tau+\Delta x^{\prime}}\left[E_{y^{\prime}}^{n}\left(0, l+\frac{1}{2}, m\right)+E_{y^{\prime}}^{n}\left(1, l+\frac{1}{2}, m\right)\right]+ \\
& +\frac{\Delta x^{\prime} \tau^{2} c^{2}}{2\left(\Delta y^{\prime}\right)^{2}\left(c \tau+\Delta x^{\prime}\right)}\left[E_{y^{\prime}}^{n}\left(0, l+\frac{3}{2}, m\right)-2 E_{y^{\prime}}^{n}\left(0, l+\frac{1}{2}, m\right)+E_{y^{\prime}}^{n}\left(0, l-\frac{1}{2}, m\right)+\right. \\
& \left.+E_{y^{\prime}}^{n}\left(1, l+\frac{3}{2}, m\right)-2 E_{y^{\prime}}^{n}\left(1, l+\frac{1}{2}, m\right)+E_{y^{\prime}}^{n}\left(1, l-\frac{1}{2}, m\right)\right]+ \\
& +\frac{\Delta x^{\prime} \tau^{2} c^{2}}{2\left(\Delta z^{\prime}\right)^{2}\left(c \tau+\Delta x^{\prime}\right)}\left[E_{y^{\prime}}^{n}\left(0, l+\frac{1}{2}, m+1\right)-2 E_{y^{\prime}}^{n}\left(0, l+\frac{1}{2}, m\right)+E_{y^{\prime}}^{n}\left(0, l+\frac{1}{2}, m-1\right)+\right. \\
& \left.+E_{y^{\prime}}^{n}\left(1, l+\frac{1}{2}, m+1\right)-2 E_{y^{\prime}}^{n}\left(1, l+\frac{1}{2}, m\right)+E_{y^{\prime}}^{n}\left(1, l+\frac{1}{2}, m-1\right)\right] .
\end{aligned}
$$

The discretized ABCs for all the field components on the remaining planes can be derived from (3.54).

Proposition 3.4 (Consistency of Yee's scheme). Yee's scheme is consistent in order two in space and time.

Proof. As seen from (3.45) and (3.46), Yee's scheme employs the first order central difference approximation for temporal and spatial derivatives. It is well-known that such approximation is consistent at order 2. Indeed, for some smooth enough function $\Phi: \mathbb{R}^{3} \times \mathbb{R}_{+} \rightarrow \mathbb{R}^{6}$, we have for each spatial and temporal derivative same as for $x^{\prime}$ :

$$
\left(\partial_{x^{\prime}} \Phi\right)_{i}=\frac{\Phi_{i+1}-\Phi_{i-1}}{2 \Delta x^{\prime}}+\mathcal{O}\left(\Delta x^{\prime 2}\right), \quad i \geq 1
$$

since

$$
\begin{aligned}
\Phi_{i+1} & =\Phi_{i}+\left(\partial_{x^{\prime}} \Phi\right)_{i} \Delta x^{\prime}+\left(\partial_{x^{\prime} x^{\prime}}^{2} \Phi\right)_{i} \frac{\Delta x^{\prime 2}}{2}+\left(\partial_{x^{\prime} x^{\prime} x^{\prime}}^{3} \Phi\right)_{i} \frac{\Delta x^{\prime 3}}{6}+\mathcal{O}\left(\Delta x^{\prime 4}\right) \\
\Phi_{i-1} & =\Phi_{i}-\left(\partial_{x^{\prime}} \Phi\right)_{i} \Delta x^{\prime}+\left(\partial_{x^{\prime} x^{\prime}}^{2} \Phi\right)_{i} \frac{\Delta x^{\prime 2}}{2}-\left(\partial_{x^{\prime} x^{\prime} x^{\prime}}^{3} \Phi\right)_{i} \frac{\Delta x^{\prime 3}}{6}+\mathcal{O}\left(\Delta x^{\prime 4}\right)
\end{aligned}
$$

Thus, Yee's scheme has a second order truncated error in both space and time. The described above Mur's ABCs are designed to keep the same order of accuracy. 
Proposition $3.5\left(\ell^{2}\right.$-stability of Yee's scheme). In $3 d$ case Yee's scheme is $\ell^{2}$ stable under $3 d$ CFL-condition:

$$
c \tau\left(\frac{1}{\Delta x^{\prime 2}}+\frac{1}{\Delta y^{\prime 2}}+\frac{1}{\Delta z^{\prime 2}}\right)^{\frac{1}{2}}<1
$$

Proof. To prove stability of the 3d Yee's scheme for homogeneous MEs (3.46), (3.45), i.e. when $P_{x^{\prime}}^{n-\frac{1}{2}}\left(k+\frac{1}{2}, l, m\right), P_{y^{\prime}}^{n-\frac{1}{2}}\left(k, l+\frac{1}{2}, m\right), J_{x^{\prime}}^{n-\frac{1}{2}}\left(k+\frac{1}{2}, l, m\right) J_{y^{\prime}}^{n-\frac{1}{2}}\left(k, l+\frac{1}{2}, m\right)$ and $J_{z^{\prime}}^{n-\frac{1}{2}}\left(k, l, m+\frac{1}{2}\right)$ are zeros, we will follow the procedure given in [23], for Yee's scheme implemented $1 \mathrm{~d}$ and $2 \mathrm{~d}$ MEs. We start by computing the amplification matrix $G$, such that $U^{n}=G U^{n-1}$, and $U_{k l m}^{n}:=U^{n} e^{\mathrm{i}(\zeta k+\eta l+\theta m)}$ with $(\zeta, \eta, \theta) \in(-\pi, \pi)^{3}$. We assign the field-vector with two staggered iterations $n-1$ and $n-\frac{1}{2}$ as

$$
\begin{aligned}
& U_{k l m}^{n-1}=\left(E_{x^{\prime}}^{n-1}\left(k+\frac{1}{2}, l, m\right), E_{y^{\prime}}^{n-1}\left(k, l+\frac{1}{2}, m\right), E_{z^{\prime}}^{n-1}\left(k, l, m+\frac{1}{2}\right),\right. \\
& \left.\quad B_{x^{\prime}}^{n-\frac{1}{2}}\left(k, l+\frac{1}{2}, m+\frac{1}{2}\right), B_{y^{\prime}}^{n-\frac{1}{2}}\left(k+\frac{1}{2}, l, m+\frac{1}{2}\right), B_{z^{\prime}}^{n-\frac{1}{2}}\left(k+\frac{1}{2}, l+\frac{1}{2}, m\right)\right),
\end{aligned}
$$

then after one iteration the vector is defined as

$$
\begin{aligned}
& U_{k l m}^{n}=\left(E_{x^{\prime}}^{n}\left(k+\frac{1}{2}, l, m\right), E_{y^{\prime}}^{n}\left(k, l+\frac{1}{2}, m\right), E_{z^{\prime}}^{n}\left(k, l, m+\frac{1}{2}\right),\right. \\
& \left.\quad B_{x^{\prime}}^{n+\frac{1}{2}}\left(k, l+\frac{1}{2}, m+\frac{1}{2}\right), B_{y^{\prime}}^{n+\frac{1}{2}}\left(k+\frac{1}{2}, l, m+\frac{1}{2}\right), B_{z^{\prime}}^{n+\frac{1}{2}}\left(k+\frac{1}{2}, l+\frac{1}{2}, m\right)\right) .
\end{aligned}
$$

First, we rewrite the numerical schemes (3.46), (3.45) into the matrix form $A U_{k l m}^{n}=$ $B U_{k l m}^{n-1}$, where

$$
A_{6 \times 6}=\left[\begin{array}{cc}
I_{3 \times 3} & 0_{3 \times 3} \\
L_{3 \times 3} & I_{3 \times 3}
\end{array}\right] \text { and } B_{6 \times 6}=\left[\begin{array}{cc}
I_{3 \times 3} & M_{3 \times 3} \\
0_{3 \times 3} & I_{3 \times 3}
\end{array}\right]
$$

are two block-matrices, consisting of the identity matrices $I_{3 \times 3}$, the zero matrices $0_{3 \times 3}$, and two other matrices: 


$$
L=\left[\begin{array}{ccc}
0 & -\lambda_{3}\left(e^{\mathrm{i} \theta}-1\right) & \lambda_{2}\left(e^{\mathrm{i} \eta}-1\right) \\
\lambda_{3}\left(e^{\mathrm{i} \theta}-1\right) & 0 & -\lambda_{1}\left(e^{\mathrm{i} \zeta}-1\right) \\
-\lambda_{2}\left(e^{\mathrm{i} \eta}-1\right) & \lambda_{1}\left(e^{\mathrm{i} \zeta}-1\right) & 0
\end{array}\right]
$$

and

$$
M=\left[\begin{array}{ccc}
0 & -\lambda_{3}\left(1-e^{-i \theta}\right) & \lambda_{2}\left(1-e^{-i \eta}\right) \\
\lambda_{3}\left(1-e^{-i \theta}\right) & 0 & -\lambda_{1}\left(1-e^{-i \zeta}\right) \\
-\lambda_{2}\left(1-e^{-i \eta}\right) & \lambda_{1}\left(1-e^{-i \zeta}\right) & 0
\end{array}\right]
$$

for which we used notation

$$
\lambda_{1}=c \tau / \Delta x^{\prime}, \quad \lambda_{2}=c \tau / \Delta y^{\prime}, \quad \lambda_{3}=c \tau / \Delta z^{\prime} .
$$

Notice some useful properties of $L$ and $M: M^{T}=-M, L^{T}=-L, L=-M^{*}$.

$$
\begin{aligned}
& \text { We easily see that } A^{-1}=\left[\begin{array}{cc}
I_{3 \times 3} & 0_{3 \times 3} \\
-L_{3 \times 3} & I_{3 \times 3}
\end{array}\right] \text { and hence } \\
& \qquad G=A^{-1} B=\left[\begin{array}{cc}
I & M \\
-L & I-L M
\end{array}\right]=\left[\begin{array}{cc}
I & M \\
M^{*} & I+M^{*} M
\end{array}\right],
\end{aligned}
$$

where $I+M^{*} M=$

$$
\left[\begin{array}{ccc}
1-4 \lambda_{2}^{2} \sin ^{2} \frac{\eta}{2}-4 \lambda_{3}^{2} \sin ^{2} \frac{\theta}{2} & -\lambda_{1} \lambda_{2}\left(1-e^{-i \zeta}\right)\left(e^{i \eta}-1\right) & -\lambda_{1} \lambda_{3}\left(1-e^{-i \zeta}\right)\left(e^{i \theta}-1\right) \\
-\lambda_{1} \lambda_{2}\left(e^{i \zeta}-1\right)\left(1-e^{-i \eta}\right) & 1-4 \lambda_{1}^{2} \sin ^{2} \frac{\zeta}{2}-4 \lambda_{3}^{2} \sin ^{2} \frac{\theta}{2} & -\lambda_{2} \lambda_{3}\left(e^{\mathrm{i} \theta}-1\right)\left(1-e^{-i \eta}\right) \\
-\lambda_{1} \lambda_{3}\left(e^{\mathrm{i} \zeta}-1\right)\left(1-e^{-\mathrm{i} \theta}\right) & -\lambda_{2} \lambda_{3}\left(1-e^{-\mathrm{i} \theta}\right)\left(e^{\mathrm{i} \eta}-1\right) & 1-4 \lambda_{1}^{2} \sin ^{2} \frac{\zeta}{2}-4 \lambda_{2}^{2} \sin ^{2} \frac{\eta}{2}
\end{array}\right]
$$

From (3.62), one can see that $G$ is an Hermitian matrix: $G^{T}=G^{*}$, so all its eigenvalues are real. The computations show that the characteristic polynomial of $G$ can be expressed in the form

$$
\varphi(Z)=(1-Z)^{2} P_{4}(Z)
$$


where $P_{4}(Z)$ denotes the quadric polynomial

$$
P_{4}(Z)=Z^{4}+2 a Z^{3}+\left(a^{2}-b\right) Z^{2}+a b Z-b-1
$$

with coefficients

$$
\begin{aligned}
& a=4\left(\lambda_{1}^{2} \sin ^{2} \frac{\zeta}{2}+\lambda_{2}^{2} \sin ^{2} \frac{\eta}{2}+\lambda_{3}^{2} \sin ^{2} \frac{\theta}{2}\right)-2, \\
& b=2\left(1-4 \lambda_{3}^{2} \sin ^{2} \frac{\theta}{2}\right) .
\end{aligned}
$$

Thus, the sum of the all eigenvalues of $G$ except $Z_{1}=Z_{2}=1$ is $\sum_{i=3}^{6} Z_{i}=-2 a$, while their product $\operatorname{det}(G)=\prod_{i=1}^{6} Z_{i}=b-1$. The necessary stability condition of the scheme requires that all the roots $\left|Z_{i}\right| \leq 1(i=1,2 \ldots 6)[23,113]$. With this aim we set bounds on the sum of the rest 4 roots

$$
-4<4-8\left(\lambda_{1}^{2} \sin ^{2} \frac{\zeta}{2}+\lambda_{2}^{2} \sin ^{2} \frac{\eta}{2}+\lambda_{3}^{2} \sin ^{2} \frac{\theta}{2}\right)<4
$$

The LHS inequality follows the necessary CFL-condition for 3d problem, back to notation (3.61) it is (3.56). Thus the characteristic polynomial of the amplification matrix $G, \varphi(Z)$, is von Neumann polynomial under the CFL-condition (3.56). However, $\varphi(Z)$ has $Z=1$ as its double root, i.e. it is not a simple von Neumann polynomial and by the theorem 4.2.1 from [113], we cannot guarantee the fulfillment of the sufficiency condition by itself. To prove that condition (3.56) is nevertheless sufficient for the stability of the discussed $3 \mathrm{~d}$ scheme, we employ the eigendecomposition of the hermitian amplification matrix: $G=Q \Lambda Q^{-1}$, where $\Lambda=\operatorname{diag}\left(Z_{1}, Z_{2}, \ldots Z_{6}\right)$ is real-valued, and the columns of the unitary matrix $Q$ are the eigenvectors of $G$. Then, as $G$ does not depend on time explicitly, one can conclude that $U^{n}=G U^{n-1}=G^{n} U^{0}$, with $G^{n}=Q \Lambda^{n} Q^{-1}$. Obviously, for any iteration $n$, all entries of this matrix are bounded and all eigenvalues are less or equal than 1, which provides the stability of the scheme. 


\subsection{Discretization of the 2d Schrödinger Equation}

Because of the relatively low gas density, it is reasonable to neglect the interactions between $\mathrm{H}_{2}^{+}$-ions. For example, at pressure of 13 atmospheres, which corresponds to density $\mathcal{N}_{0}=5.17 \times 10^{-5} a_{0}^{-3}$, the average distance between $\mathrm{H}_{2}^{+}$-molecules can be estimated to as 27 a.u. (1.4 nm) exceeding by an order of magnitude the molecular size $R_{0}=2$ a.u. In other words, we can solve each of the TDSE independently, and with such reasoning we omit in this section the index $i$ in the second equation of the system (2.2), writing it simply as:

$$
\mathrm{i} \partial_{t} \psi(\mathbf{x}, t)=-\frac{1}{2} \triangle_{\mathbf{x}} \psi(\mathbf{x}, t)+V_{C}\left(\mathbf{x}, R_{0}\right) \psi(\mathbf{x}, t)+\mathbf{x} \cdot \mathbf{E}_{\mathbf{x}^{\prime}}(t) \psi(\mathbf{x}, t)
$$

with the same notation for the Coulomb potential $V_{C}$ and the internuclear distance $R_{0}$.

\subsubsection{Symmetric Strang Splitting}

Equation (3.64) is solved using Strang's splitting [111] in time, which in case of 2dTDSE reads [85]:

$$
\left\{\begin{array}{c}
\partial_{t} \psi(x, y, t)=-i\left(x E_{\mathbf{x}^{\prime}, x}(t)+y E_{\mathbf{x}^{\prime}, y}(t)\right) \psi(x, y, t), \\
t \in\left[t_{n}, t_{n+1 / 2}\right], \quad \psi\left(x, y, t_{n}\right)=\psi^{n}(x, y), \\
\partial_{t} \psi(x, y, t)=\frac{i}{2} \triangle_{x, y} \psi(x, y, t)-i V_{C}\left(x, y, R_{0}\right) \psi(x, y, t), \\
t \in\left[t_{n}, t_{n^{*}+1}\right], \quad \psi\left(x, y, t_{n}\right)=\psi^{n+1 / 2}(x, y), \\
\partial_{t} \psi(x, y, t)=-i\left(x E_{\mathbf{x}^{\prime}, x}(t)+y E_{\mathbf{x}^{\prime}, y}(t)\right) \psi(x, y, t), \\
t \in\left[t_{n+1 / 2}, t_{n+1}\right], \quad \psi\left(x, y, t_{n+1 / 2}\right)=\psi^{n^{*}+1}(x, y) .
\end{array}\right.
$$

The illustration for the method is given on the Fig.3.3. We have denoted by $\psi^{n}, \psi^{n+1 / 2}$ the space-dependent wavefunction at time $t_{n}, t_{n+1 / 2}$. The first step (I) provides an- 


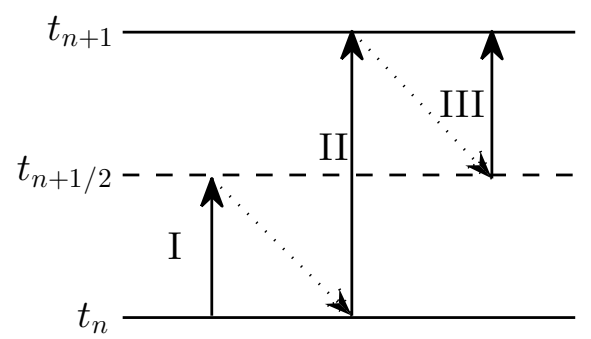

FiguRE 3.3: Symmetry Strang splitting scheme.

alytically $\psi^{n+1 / 2}=\psi^{n} e^{-\mathrm{i}\left(x E_{\mathbf{x}^{\prime}, x}^{n}+y E_{\mathbf{x}^{\prime}, y}^{n}\right) \Delta t_{S} / 2}$, where $\Delta t_{S}=t_{n+1}-t_{n}=t_{n^{*}+1}-t_{n}=$ $2\left(t_{n+1}-t_{n+1 / 2}\right)=2\left(t_{n+1 / 2}-t_{n}\right)$. In the second step (II), the corresponding laser-free TDSE with initial data $\psi^{n+1 / 2}$ is numerically solved (see Section 3.3.2), and finally, the third step (III) is solved as the first one: $\psi^{n+1}=\psi^{n^{*}+1} e^{-\mathbf{i}\left(x E_{\mathbf{x}^{\prime}, x}^{n+1 / 2}+y E_{\mathbf{x}^{\prime}, y}^{n+1 / 2}\right) \Delta t_{S} / 2}$.

Proposition 3.6 (Local Error in Strang's Splitting). Symmetric Strang's splitting (3.65) has consistency order 2.

Proof. For the initial value problem

$$
u_{t}=A u+B u, \quad u(0)=u_{0}
$$

where $A$ and $B$ are two noncommutative operators, the difference between the exact solution $u(t)$ and the solution obtained by using Strang splitting method $u^{(S)}(t)$ (so that, $u^{(S)}(t+\Delta t)=e^{(\Delta t / 2) A} e^{\Delta t B} e^{(\Delta t / 2) A} u(t)$ ) is given by the Backer-CampbellHausdorff formula

$$
u^{(S)}(t)-u(t)=\Delta t^{3}\left(\frac{1}{12}[B,[B, A]]-\frac{1}{24}[A,[A, B]]\right) u(t)+\mathcal{O}\left(\Delta t^{4}\right)
$$

In our case, see (3.65), the operators $A=-\mathbf{i x} \cdot \mathbf{E}_{\mathbf{x}^{\prime}}(t)$ and $B=\mathbf{i}\left(\frac{1}{2} \triangle_{\mathbf{x}}-V_{C}(\mathbf{x})\right)$, so the commutator $[A, B]=-\frac{1}{2} \triangle_{\mathbf{x}}\left(\mathbf{x} \cdot \mathbf{E}_{\mathbf{x}^{\prime}}(t)\right)-\nabla_{\mathbf{x}}\left(\mathbf{x} \cdot \mathbf{E}_{\mathbf{x}^{\prime}}(t)\right) \cdot \nabla_{\mathbf{x}}$, and since $\mathbf{E}_{\mathbf{x}^{\prime}}(t)$ does not depend on $\mathbf{x}$ we get $[A, B]=-\mathbf{E}_{\mathbf{x}^{\prime}}(t) \cdot \nabla_{\mathbf{x}}$. Further we compute the desired 
commutators

$$
\begin{aligned}
& {[A,[A, B]]=-\mathrm{i}\left(\mathbf{E}_{\mathbf{x}^{\prime}}(t) \cdot \nabla_{\mathbf{x}}\right)\left(\mathbf{x} \cdot \mathbf{E}_{\mathbf{x}^{\prime}}(t)\right)=-\mathrm{i} \mathbf{E}_{\mathbf{x}^{\prime}}^{2}(t),} \\
& {[B,[B, A]]=\mathrm{i}\left(\frac{1}{4} \triangle_{\mathbf{x}}^{2}\left(\mathbf{x} \cdot \mathbf{E}_{\mathbf{x}^{\prime}}(t)\right)-\left(\nabla_{\mathbf{x}}\left(\mathbf{x} \cdot \mathbf{E}_{\mathbf{x}^{\prime}}(t)\right) \cdot\left(\nabla_{\mathbf{x}} V_{C}(\mathbf{x})\right)-\right.\right.} \\
& \left.\quad-\triangle_{\mathbf{x}}\left(\mathbf{x} \cdot \mathbf{E}_{\mathbf{x}^{\prime}}(t)\right) \triangle_{\mathbf{x}}+\triangle_{\mathbf{x}} \nabla_{\mathbf{x}}\left(\mathbf{x} \cdot \mathbf{E}_{\mathbf{x}^{\prime}}(t)\right) \nabla_{\mathbf{x}}\right)=\mathrm{i} \mathbf{E}_{\mathbf{x}^{\prime}}(t) \cdot \nabla_{\mathbf{x}} V_{C}(\mathbf{x}) .
\end{aligned}
$$

Now we can estimate the error as

$$
\begin{aligned}
& \left|\psi^{(S)}(x, y, t+\Delta t)-\psi^{(E)}(x, y, t+\Delta t)\right| \leq \\
& \Delta t_{S}^{3}\left(\frac{1}{24}\left|\mathbf{E}_{\mathbf{x}^{\prime}}^{2}(t) \psi^{(E)}(x, y, t)\right|+\frac{1}{12}\left|\mathbf{E}_{\mathbf{x}^{\prime}}(t) \cdot \nabla_{\mathbf{x}} V_{C}(\mathbf{x}) \psi^{(E)}(x, y, t)\right|\right) .
\end{aligned}
$$

Thus, locally (at each time iteration) the Strang splitting has a $3^{\text {rd }}$ order truncation error in time. However, the Coulomb potential $V_{C}(\mathbf{x})=-\frac{1}{\left|\mathbf{x}-\mathbf{R}_{0} / 2\right|}-\frac{1}{\left|\mathbf{x}+\mathbf{R}_{0} / 2\right|}$, where the vector $\mathbf{R}_{0}$ takes account of orientation of the molecule in space, hence $\nabla_{\mathbf{x}} V_{C}(\mathbf{x})=\frac{\mathbf{x}-\mathbf{R}_{0} / 2}{\left|\mathbf{x}-\mathbf{R}_{0} / 2\right|^{3}}+\frac{\mathbf{x}+\mathbf{R}_{0} / 2}{\left|\mathbf{x}+\mathbf{R}_{0} / 2\right|^{3}}$, so that the closer to the one of nuclei, the higher contribution from the second term of RHS in (3.68). Notice, that globally (for all propagation time) the error has a $2^{\text {nd }}$ order [77]. 


\subsubsection{Numerical Scheme for the Schrödinger Equation with Time-independent Potential}

Here we describe how to solve of the second equation in the system (3.65), i.e. $2 \mathrm{~d}$ TDSE. On assumption that the spatial domain $\omega$ is large enough, we set for now zero boundary conditions:

$$
\left\{\begin{array}{l}
\partial_{t} \psi(x, y, t)=\frac{i}{2} \triangle_{x, y} \psi(x, y, t)-i V_{C}(x, y) \psi(x, y, t), \quad(x, y) \in \omega, t>0 \\
\psi(x, y, 0)=\psi_{0}(x, y), \quad(x, y) \in \omega \\
\psi(x, y, t)=0, \quad(x, y) \in \partial \omega, t>0
\end{array}\right.
$$

where $\omega=(a, b) \times(c, d) \in \mathbb{R}^{2}$. Let us define the space steps in $x$ and $y$ as $h_{x}=$ $(b-a) /\left(N_{x}+1\right)$ and $h_{y}=(d-c) /\left(N_{y}+1\right)$, where $N_{x}, N_{y} \geq 1$. Then internal points $x_{j}=a+j h_{x}\left(1 \leq j \leq N_{x}\right)$ and $y_{m}=c+m h_{y}\left(1 \leq m \leq N_{y}\right)$, while on the boundaries: $x_{0}=a, x_{N_{x}+1}=b, y_{0}=c$ and $y_{N_{y}+1}=d$. Then we denote $\psi_{j, m}(t)=\psi\left(x_{j}, y_{m}, t\right)$ and recall that for all $\psi \in \mathcal{C}^{2}\left(\omega \times \mathbb{R}_{+}\right)$the discretization

$$
\begin{aligned}
\triangle \psi\left(x_{j}, y_{m}, t\right) & =\frac{\psi_{j+1, m}-2 \psi_{j, m}+\psi_{j-1, m}}{h_{x}^{2}}+ \\
& +\frac{\psi_{j, m+1}-2 \psi_{j, m}+\psi_{j, m-1}}{h_{y}^{2}}+\mathcal{O}\left(\max \left[h_{x}^{2}, h_{y}^{2}\right]\right)
\end{aligned}
$$

is consistent at order 2 both in $x$ and $y$ (see further subsection 3.8). As $\psi$ also depends on time, we denote the time step $k$, such that $t_{n}=k n(n \geq 0)$, and introduce $\psi_{j, m}^{n}=\psi\left(x_{j}, y_{m}, t_{n}\right)$. The Crank-Nicolson scheme for $2 \mathrm{~d}$ Schrödinger equation in (3.69) reads

$$
\begin{array}{r}
\frac{\psi_{j, m}^{n+1}-\psi_{j, m}^{n}}{k}=\frac{i\left(\psi_{j+1, m}^{n+1}-2 \psi_{j, m}^{n+1}+\psi_{j-1, m}^{n+1}+\psi_{j+1, m}^{n}-2 \psi_{j, m}^{n}+\psi_{j-1, m}^{n}\right)}{4 h_{x}^{2}}+ \\
+\frac{i\left(\psi_{j, m+1}^{n+1}-2 \psi_{j, m}^{n+1}+\psi_{j, m-1}^{n+1}+\psi_{j, m+1}^{n}-2 \psi_{j, m}^{n}+\psi_{j, m-1}^{n}\right)}{4 h_{y}^{2}}- \\
-i V_{C}\left(x_{j}, y_{m}\right) \frac{\left(\psi_{j, m}^{n+1}+\psi_{j, m}^{n}\right)}{2}
\end{array}
$$


with boundary and initially conditions:

$$
\begin{aligned}
& \psi_{0, m}^{n}=\psi_{N_{x}+1, m}^{n}=0, \\
& \psi_{j, 0}^{n}=\psi_{j, N_{y}+1}^{n}=0, \\
& \psi_{j, m}^{0}=\left(\psi_{0}\right)_{j, m} .
\end{aligned}
$$

Later, in propositions 3.8 and 3.9, we state convergence and stability of the numerical scheme (3.71).

The standard method of transformation the schemes like (3.71) given on rectangular domains is the Alternative Direction Implicit (ADI) method [113]. We introduce the new space grid $M_{l}$ with the internal points (excluding the domain boundary) by using the unique index $l=j+(m-1) N_{x}: M_{1}=\left(x_{1}, y_{1}\right), M_{2}=\left(x_{2}, y_{1}\right), \ldots$, $M_{N_{x}}=\left(x_{N_{x}}, y_{1}\right), M_{N_{x}+1}=\left(x_{1}, y_{2}\right), \ldots, M_{N_{x} \times N_{y}}=\left(x_{N_{x}}, y_{N_{y}}\right)$. Fig. 3.4 shows the new mesh, where the internal points $M_{l}$ are represented by black dots. The boundary points, where the wave function is assumed to approach 0, are shown as disks.

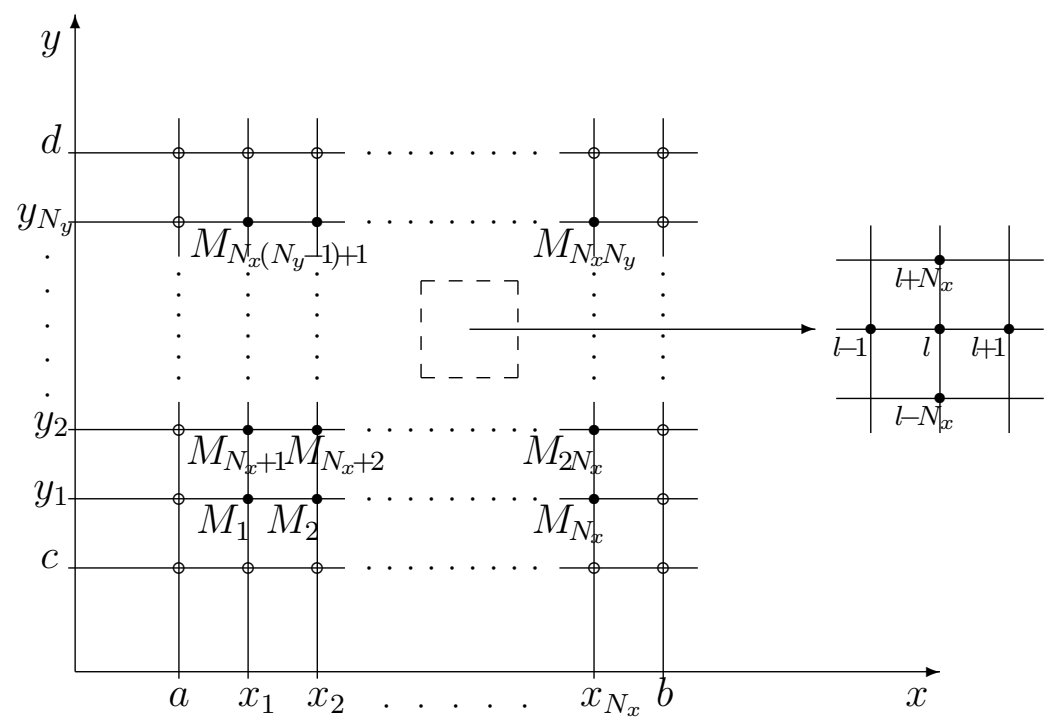

Figure 3.4: New space grid: $\circ$ - boundary points and $\bullet$ - internal points, denoted $M_{l}$. The callout: 5-points discretization scheme for the internal points 
Now we rewrite the discrete equation (3.71) using indexes $l$ (for space) and $n$ (for time):

$$
\begin{aligned}
& \frac{k}{4 h_{y}^{2}} \psi_{l+N_{x}}^{n+1}+\frac{k}{4 h_{x}^{2}} \psi_{l+1}^{n+1}+\psi_{l}^{n+1}\left(i-\frac{k}{2 h_{x}^{2}}-\frac{k}{2 h_{y}^{2}}-\frac{k V_{C}\left(M_{l}\right)}{2}\right)+\frac{k}{4 h_{x}^{2}} \psi_{l-1}^{n+1}+\frac{k}{4 h_{y}^{2}} \psi_{l-N_{x}}^{n+1}= \\
& -\frac{k}{4 h_{y}^{2}} \psi_{l+N_{x}}^{n}-\frac{k}{4 h_{x}^{2}} \psi_{l+1}^{n}+\psi_{l}^{n}\left(\mathrm{i}+\frac{k}{2 h_{x}^{2}}+\frac{k}{2 h_{y}^{2}}+\frac{k V_{C}\left(M_{l}\right)}{2}\right)-\frac{k}{4 h_{x}^{2}} \psi_{l-1}^{n}-\frac{k}{4 h_{y}^{2}} \psi_{l-N_{x}}^{n} .
\end{aligned}
$$

Note that according to the BCs from (3.72) on the side

$$
\begin{array}{ll}
\mathrm{S}\left(l=2,3, \ldots, N_{x}-1\right) & : \text { all } \quad \psi_{l-N_{x}}=0 \\
\mathrm{~N}\left(l=N_{x}\left(N_{y}-1\right)+2, N_{x}\left(N_{y}-1\right)+3, \ldots, N_{x} N_{y}-1\right) & : \text { all } \quad \psi_{l+N_{x}}=0 \\
\mathrm{~W}\left(l=N_{x}+1,2 N_{x}+1, \ldots, N_{x}\left(N_{y}-2\right)+1\right) & : \text { all } \quad \psi_{l-1}=0 \\
\mathrm{E}\left(l=2 N_{x}, 3 N_{x}, \ldots, N_{x}\left(N_{y}-1\right)\right) & : \text { all } \quad \psi_{l+1}=0
\end{array}
$$

as well as at the corresponding corners of the mesh

$$
\begin{array}{ll}
\mathrm{SW}(l=1) & : \psi_{1}=0 \\
\mathrm{SE}\left(l=N_{x}\right) & : \psi_{N_{x}}=0 \\
\mathrm{NW}\left(l=N_{x}\left(N_{y}-1\right)+1\right) & : \psi_{N_{x}\left(N_{y}-1\right)+1}=0 \\
\mathrm{NE}\left(l=N_{x} N_{y}\right) & : \quad \psi_{N_{x} N_{y}}=0 .
\end{array}
$$

Using (3.73)-(3.75), one can set out the above system in the linear system form:

$$
A \psi^{n+1}=B \psi^{n}
$$

where the vectors $\psi^{n+1}, \psi^{n} \in \mathbb{C}^{N_{x} N_{y}}$, and the matrices $A_{N_{x} N_{y} \times N_{x} N_{y}}, B_{N_{x} N_{y} \times N_{x} N_{y}} \in$ $M_{N_{x} N_{y}}(\mathbb{C})$.

Let us decompose the complex wave function into real and imaginary parts:

$$
\psi=\psi_{R}+i \psi_{I},
$$


where $\psi_{R}, \psi_{I} \in \mathbb{R}^{N_{x} N_{y}}$, then for the internal points one gets:

$$
\begin{aligned}
& \frac{k}{4 h_{y}^{2}}\left(\psi_{R}\right)_{l+N_{x}}^{n+1}+\frac{k}{4 h_{x}^{2}}\left(\psi_{R}\right)_{l+1}^{n+1}-\left(\psi_{R}\right)_{l}^{n+1}\left(\frac{k}{2 h_{x}^{2}}+\frac{k}{2 h_{y}^{2}}+\frac{k V_{C}\left(M_{l}\right)}{2}\right)+ \\
& +\frac{k}{4 h_{x}^{2}}\left(\psi_{R}\right)_{l-1}^{n+1}+\frac{k}{4 h_{y}^{2}}\left(\psi_{R}\right)_{l-N_{x}}^{n+1}-\left(\psi_{I}\right)_{l}^{n+1}+\mathrm{i}\left[\frac{k}{4 h_{y}^{2}}\left(\psi_{I}\right)_{l+N_{x}}^{n+1}+\frac{k}{4 h_{x}^{2}}\left(\psi_{I}\right)_{l+1}^{n+1}-\right. \\
& \left.-\left(\psi_{I}\right)_{l}^{n+1}\left(\frac{k}{2 h_{x}^{2}}+\frac{k}{2 h_{y}^{2}}+\frac{k V_{C}\left(M_{l}\right)}{2}\right)+\frac{k}{4 h_{x}^{2}}\left(\psi_{I}\right)_{l-1}^{n+1}+\frac{k}{4 h_{y}^{2}}\left(\psi_{I}\right)_{l-N_{x}}^{n+1}+\left(\psi_{R}\right)_{l}^{n+1}\right]= \\
& -\frac{k}{4 h_{y}^{2}}\left(\psi_{R}\right)_{l+N_{x}}^{n}-\frac{k}{4 h_{x}^{2}}\left(\psi_{R}\right)_{l+1}^{n}+\left(\psi_{R}\right)_{l}^{n}\left(\frac{k}{2 h_{x}^{2}}+\frac{k}{2 h_{y}^{2}}+\frac{k V_{C}\left(M_{l}\right)}{2}\right)- \\
& -\frac{k}{4 h_{x}^{2}}\left(\psi_{R}\right)_{l-1}^{n}-\frac{k}{4 h_{y}^{2}}\left(\psi_{R}\right)_{l-N_{x}}^{n}-\left(\psi_{I}\right)_{l}^{n}-\mathrm{i}\left[\frac{k}{4 h_{y}^{2}}\left(\psi_{I}\right)_{l+N_{x}}^{n}+\frac{k}{4 h_{x}^{2}}\left(\psi_{I}\right)_{l+1}^{n}-\right. \\
& \left.-\left(\psi_{I}\right)_{l}^{n}\left(\frac{k}{2 h_{x}^{2}}+\frac{k}{2 h_{y}^{2}}+\frac{k V_{C}\left(M_{l}\right)}{2}\right)+\frac{k}{4 h_{x}^{2}}\left(\psi_{I}\right)_{l-1}^{n}+\frac{k}{4 h_{y}^{2}}\left(\psi_{I}\right)_{l-N_{x}}^{n}-\left(\psi_{R}\right)_{l}^{n}\right],
\end{aligned}
$$

with the applied on $\psi_{R}$ and $\psi_{I}$ boundary conditions (3.74)-(3.75). Now using decompositions

$$
A=A_{R}+\mathrm{i} A_{I}, \quad B=B_{R}+\mathrm{i} B_{I}
$$

where $A_{R}, A_{I}, B_{R}, B_{I} \in M_{N_{x} N_{y}}(\mathbb{R})$, one can rewrite $(3.76)$ in the form

$$
\begin{array}{r}
A_{R} \psi_{R}^{n+1}-A_{I} \psi_{I}^{n+1}+\mathrm{i}\left[A_{I} \psi_{R}^{n+1}+A_{R} \psi_{I}^{n+1}\right]= \\
=B_{R} \psi_{R}^{n}-B_{I} \psi_{I}^{n}+\mathrm{i}\left[B_{I} \psi_{R}^{n}+B_{R} \psi_{I}^{n}\right] .
\end{array}
$$

This equation can also be written as multiplication of matrices of size $2 N_{x} N_{y} \times 2 N_{x} N_{y}$ and vectors of length $2 N_{x} N_{y}$ :

$$
\left[\begin{array}{cc}
A_{R} & -A_{I} \\
A_{I} & A_{R}
\end{array}\right]\left[\begin{array}{l}
\psi_{R}^{n+1} \\
\psi_{I}^{n+1}
\end{array}\right]=\left[\begin{array}{cc}
B_{R} & -B_{I} \\
B_{I} & B_{R}
\end{array}\right]\left[\begin{array}{l}
\psi_{R}^{n} \\
\psi_{I}^{n}
\end{array}\right]
$$

From comparison with (3.78), one can notice that $A_{I}=B_{I}=I_{N_{x} N_{y} \times N_{x} N_{y}}$ (the identity matrix of size $\left.M_{N_{x} N_{y}}(\mathbb{R})\right)$ and $B_{R}=-A_{R}$ where $A_{R}$ is penta-diagonal matrix that structure represented on Figs. 3.5 and 3.6. 


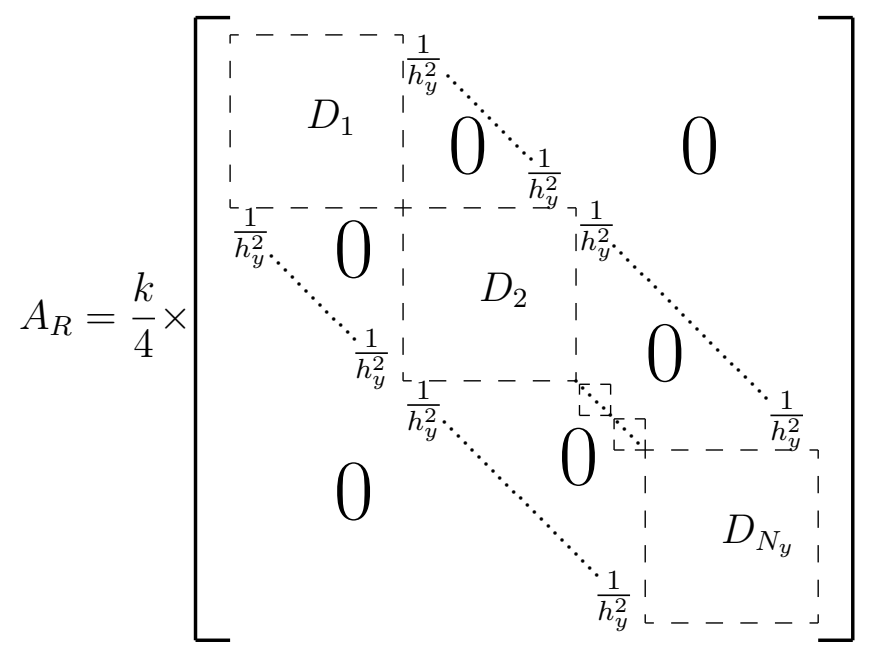

Figure 3.5: Structure of the matrix $A_{R}$ : penta-diagonal and symmetric matrix size of $N_{x} N_{y} \times N_{x} N_{y}$. On the principal diagonal of the matrix there are $N_{y}$ of the tri-diagonal submatrices $D_{m}\left(m=1, \ldots N_{y}\right)$ size of $N_{x} \times N_{x}$ (see Fig.3.6), also non-diagonal elements $\left(A_{R}\right)_{i, i+N_{x}}=\left(A_{R}\right)_{i+N_{x}, i}=k / 4 h_{y}^{2}$.

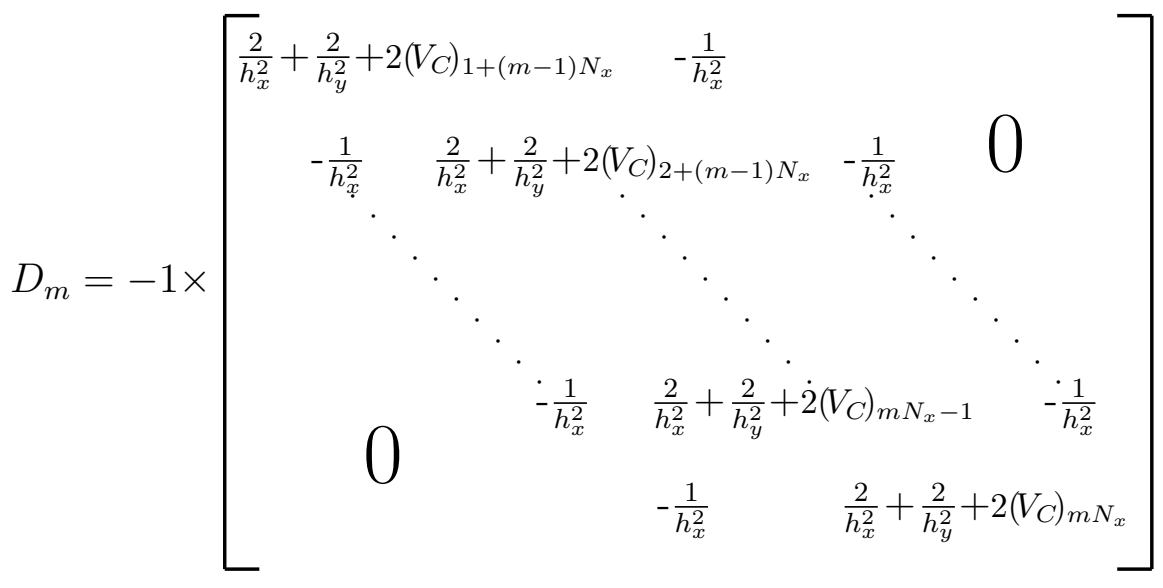

Figure 3.6: Structure of the matrixes $a_{R}$ : tri-diagonal and symmetric matrix size of $N_{x} \times N_{x}$. Diagonal elements $\left(D_{m}\right)_{i, i}=-2\left(1 / h_{x}^{2}+1 / h_{y}^{2}+V_{C}\left(M_{i+(m-1) N_{x}}\right)\right)$, non-diagonal elements $\left(D_{m}\right)_{i, i+1}=\left(D_{m}\right)_{i+1, i}=1 / h_{x}^{2}$. 


\subsubsection{Boundary Condition Problem}

In system (3.69), the initial wavefunction $\psi_{0}$ of the electron in a $\mathrm{H}_{2}^{+}$-molecule is localized within several a.u. that is comparable with the internuclear distance $R_{0} \approx 2-3.2$ a.u. However, after the interaction between a intensive laser pulse and the molecule, the wavepacket can be spread up to several thousands of a.u. In other words, to satisfy Dirichlet zero BCs one must use a huge grids with several thousand nodes. However, in practice, much smaller boxes/grids with absorbing boundary conditions (ABCs) are used [31]. Although sufficiently precise unconditionally stable discretization schemes have been developed to avoid reflection in the case of 1d-Schrödinger equation [3], these schemes are still computationally costly for 2d-equation. An empirical method is to use a complex absorbing potential (CAP) [47]. Such a potential helps to collect spirious reflections of the wavefunction at the boundary. The general idea of the method is referred to the $1 \mathrm{~d}$ problem of wavefunction transmission, reflection and absorption, for a complex potential given by:

$$
U_{a b s}(x)=\left\{\begin{array}{cl}
V_{1}-\mathrm{i} V_{2}, \quad x \in[0, a], & a>0 \\
0, & \text { otherwise }
\end{array}\right.
$$

Here the coefficient $V_{1}$ can be either positive, representing the "height" of an absorptive "barrier" or negative, representing the "depth" of an absorptive "well", while the coefficient $V_{2}$, the "absorptive strength", must be only positive. Thus, the 1d-timeindependent Schrödinger equation for the electron with energy $E$ writes

$$
-\frac{1}{2} \partial_{x}^{2} \psi=\left[E-U_{a b s}(x)\right] \psi
$$


Defining $k=\sqrt{2 E}$ and $\mu=\sqrt{k^{2}-2 U_{a b s}}$, one can write the solution as

$$
\left\{\begin{array}{l}
\psi_{I}(x)=e^{i k x}+B e^{-i k x}, \quad x<0 \\
\psi_{I I}(x)=C e^{i \mu x}+D e^{-i \mu x}, \quad a>x>0 \\
\psi_{I I I}(x)=F e^{i k x}, \quad x>a
\end{array}\right.
$$

Matching these wave-functions and their derivatives at $x=0$ and $x=a$, one finds the reflection amplitude:

$$
B=\frac{\left(1-(k / \mu)^{2}\right)\left(1-e^{-2 \mathrm{i} \mu a}\right)}{(1+k / \mu)^{2} e^{-2 \mathrm{i} \mu a}-(1-k / \mu)^{2}}
$$

as well as the transmission amplitude:

$$
F=\frac{4(k / \mu) e^{-\mathbf{i}(k+\mu) a}}{(1+k / \mu)^{2} e^{-2 i \mu a}-(1-k / \mu)^{2}} .
$$

While building the accurate absorbing BCs, we are interested in the minimum reflection $R=|B|^{2}$, and the maximum transmission $T=|F|^{2}$ coefficients. Let us introduce $A=1-R-T$, the absorption coefficient that is positive for an "absorptive potential" $\left(V_{2}>0\right)$ as in that case $T+R<1$. For illustration, see Fig. 3.7, where the reflection, transmission and absorption coefficients are represented in the cases of (i) pure absorptive potential, i.e. $V_{1}=0$, (ii) absorptive well, $V_{1}<0$, and (iii) absorptive barrier, $V_{1}>0$.

From Fig. 3.7, one can see that for the energy region of interest, the pure absorptive potential as well as the absorptive well, would be more appropriate, than the absorptive barrier, to reduce the wave-function reflection at the domain boundary. Also note that, say for energy $E=0.2$ a.u., the de Broglie wavelength $\lambda_{B}=2 \pi / \sqrt{2 E} \approx 10$ a.u. This means, that for an effective absorption of the wavefunction with such translation energy, one should choose $a \geq 10$ a.u. [47]. On the other side, in order to reduce the computational cost, one wishes to take the smallest possible range of absorption 


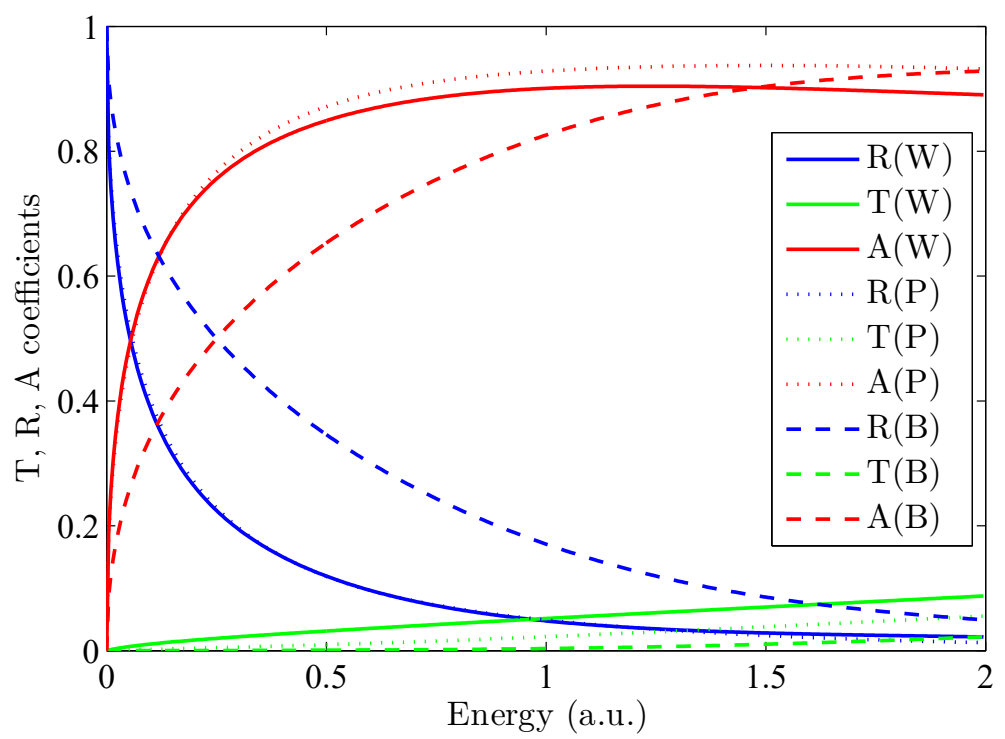

Figure 3.7: Transmission(T), Reflection(R) and Absorption(A) coefficients for cases of (i) pure(P) absorptive potential: $V_{1}=0$, (ii) absorptive well(W): $V_{1}=-1$ and (iii) absorptive barrier(B): $V_{1}=1$. For all cases the absorptive strength $V_{2}=1$, and the width of the potential $a=1.5$.

a. According to Ge and Zhang [47], the absorptive well is a better option. Indeed, in the case of purely absorptive potential, to smoothly absorb the wavefunction huge values of $a$ must be used. On the other side, in the case of absorptive well, the negative real part of the potential "helps to accelerate the downhill motion of the wavefunction by increasing its kinetic energy and reducing its de Broglie wavelength during the absorption", see [47]. With the aim of smoother absorption, we opt for complex potential with linear dependence on the coordinate. Thus, in $2 \mathrm{~d}$ case on the side (see for illustration Fig.3.8):

$$
\begin{array}{ll}
\mathrm{S}: & U_{a b s}(y)=-V_{1}\left(\frac{b-y}{b}\right)-i V_{2}\left(\frac{b-y}{b}\right), \quad y \in[0, b] \\
\mathrm{N}: & U_{a b s}(y)=-V_{1}\left(\frac{y-L+b}{b}\right)-i V_{2}\left(\frac{y-L+b}{b}\right), \quad y \in[L-b, L] \\
\mathrm{W}: & U_{a b s}(x)=-V_{1}\left(\frac{b-x}{b}\right)-i V_{2}\left(\frac{b-x}{b}\right), \quad x \in[0, b] \\
\mathrm{E}: & U_{a b s}(x)=-V_{1}\left(\frac{x-L+b}{b}\right)-i V_{2}\left(\frac{x-L+b}{b}\right), \quad x \in[L-b, L] .
\end{array}
$$




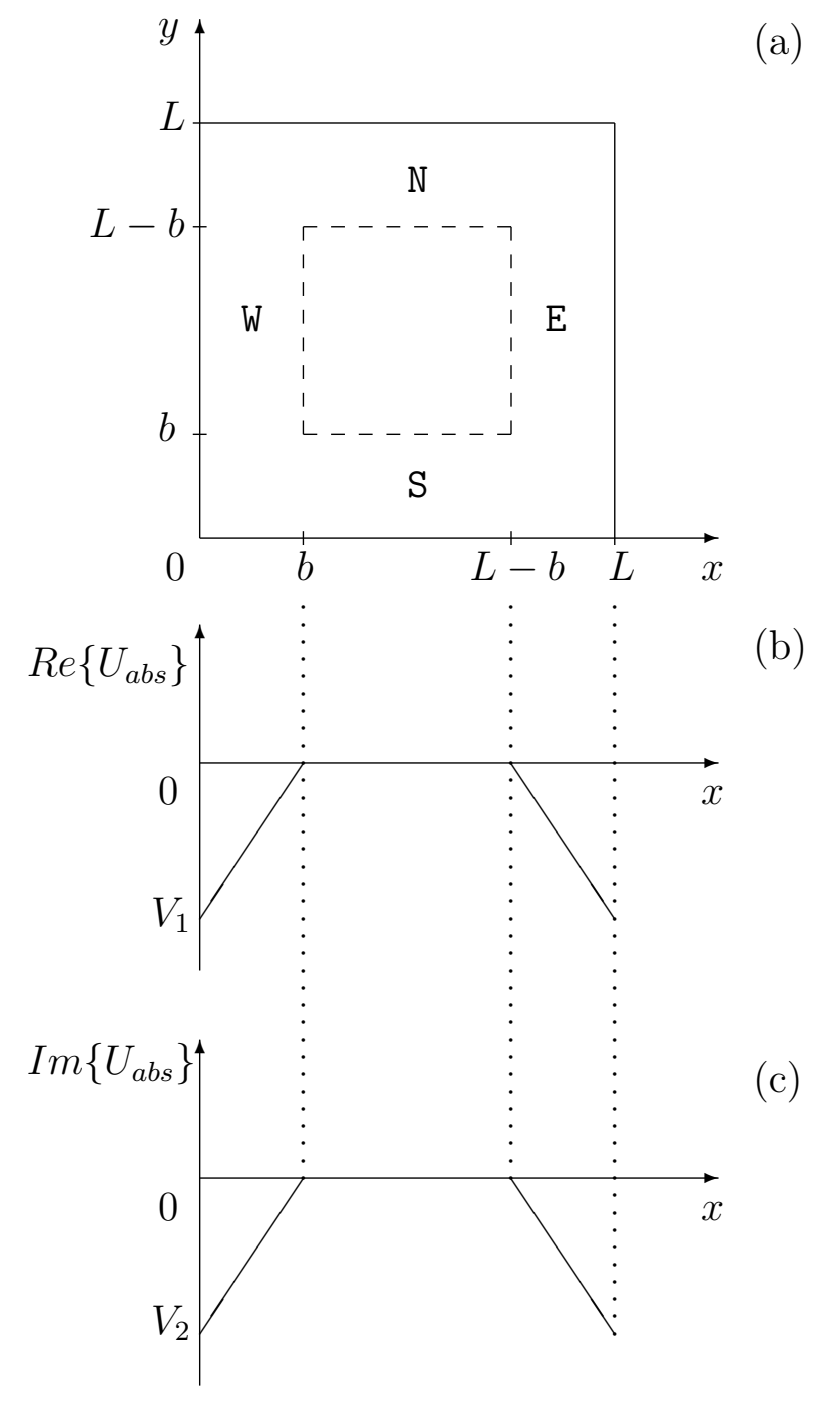

Figure 3.8: (a) Numerical domain with buffer zone of width $b$; (b)real and (c) imaginary parts of CAP as function of the coordinate. 
Now including CAP, we rewrite the equation (3.69):

$$
\begin{array}{r}
\partial_{t} \psi(x, y, t)=\frac{\mathrm{i}}{2} \triangle_{x, y} \psi(x, y, t)-\mathrm{i} V_{C}(x, y) \psi(x, y, t)-\mathrm{i} U_{a b s}(x, y) \psi(x, y, t), \\
(x, y) \in \Omega_{b}, t>0
\end{array}
$$

where $\Omega_{b}=\{(0, b) \cup(L-b, L)\} \times\{(0, b) \cup(L-b, L)\}$. Splitting the operator one can write down the solution in the form

$$
\Psi(x, y, t)=\left\{\begin{array}{l}
\psi(x, y, t), \quad(x, y) \in \Omega_{0}:=(b, L-b) \times(b, L-b), t>0 \\
\psi_{b}(x, y, t)=\psi(x, y, t) e^{-i U_{a b s} \delta t}, \quad(x, y) \in \Omega_{b}, t>0
\end{array}\right.
$$

where $\Omega=\Omega_{0} \cup \Omega_{b}$ and $\psi(x, y, t)$ is the solution obtained within the scheme described in Section 3.3.2.

Proposition 3.7. The local error resulting from the use of split-operator in (3.88) has $2^{\text {nd }}$ order in consistency.

Proof. The local error can be estimated in the same way as was done for Strang's splitting in Proposition 3.6, applying Backer-Campbell-Hausdorff formula. Now the difference between the solution obtained through splitting $\psi^{(S)}(t)$ and the exact $\psi(t)$ solution is

$$
\psi^{(S)}(t)-\psi(t)=\frac{\triangle t^{2}}{2}[A, B] \psi(t)+\mathcal{O}\left(\triangle t^{3}\right)
$$

where $A=-\mathrm{i} U_{a b s}$ and $B=\mathrm{i}\left(\frac{1}{2} \triangle_{\mathbf{x}}-V_{C}(\mathbf{x})\right)$. From (3.87) we obtain for the buffer zone:

$$
\begin{aligned}
& \mathrm{S}:[A, B]=\frac{1}{b}\left(V_{1}+i V_{2}\right) \partial_{y}, \quad y \in[0, b] \\
& \mathrm{N}:[A, B]=-\frac{1}{b}\left(V_{1}+i V_{2}\right) \partial_{y}, \quad y \in[L-b, L] \\
& \mathrm{W}:[A, B]=\frac{1}{b}\left(V_{1}+i V_{2}\right) \partial_{x}, \quad x \in[0, b] \\
& \mathrm{E}:[A, B]=-\frac{1}{b}\left(V_{1}+i V_{2}\right) \partial_{x}, \quad x \in[L-b, L] .
\end{aligned}
$$

Thus, we conclude the statement of the Proposition. 


\section{Proposition 3.8 (Consistency of the numerical scheme for the TDSE).}

The overall consistency of the numerical scheme for TDSE (3.64), which includes

i) Strang's splitting (3.65), ii) the scheme with a constant in time potential (3.71) and iii) the $A B C s(3.89)$, has $2^{\text {nd }}$ order in consistency.

Proof. According to the decomposition (3.77), we can rewrite (3.69) in the form $\mathcal{P} \Psi=0$, where $\Psi=\left(\psi_{R}, \psi_{I}\right)^{T}$ and the operator

$$
\mathcal{P}=\mathbf{I}_{2} \partial_{t}-\mathbf{J}_{2}\left(\frac{1}{2} \triangle_{x, y}-V_{C}(x, y)\right)
$$

where $\mathbf{I}_{2}$ is the $2 \times 2$ identity matrix and $\mathbf{J}_{2}=\left[\begin{array}{cc}0 & -1 \\ 1 & 0\end{array}\right]$. The discrete operator approximating $\mathcal{P}$ according to (3.71) reads

$$
\begin{array}{r}
\left(\mathcal{P}_{k, h_{1}, h_{2}} \Psi\right)_{j, m}^{n}=\mathbf{I}_{2} \frac{1}{k}\left(\Psi_{j, m}^{n+1}-\Psi_{j, m}^{n}\right)-\mathbf{J}_{2}\left(-\frac{V_{C}\left(x_{j}, y_{m}\right)}{2}\left(\Psi_{j, m}^{n+1}+\Psi_{j, m}^{n}\right)+\right. \\
+\frac{1}{4 h_{x}^{2}}\left(\Psi_{j+1, m}^{n+1}-2 \Psi_{j, m}^{n+1}+\Psi_{j-1, m}^{n+1}+\Psi_{j+1, m}^{n}-2 \Psi_{j, m}^{n}+\Psi_{j-1, m}^{n}\right)+ \\
\left.+\frac{1}{4 h_{y}^{2}}\left(\Psi_{j, m+1}^{n+1}-2 \Psi_{j, m}^{n+1}+\Psi_{j, m-1}^{n+1}+\Psi_{j, m+1}^{n}-2 \Psi_{j, m}^{n}+\Psi_{j, m-1}^{n}\right)\right)
\end{array}
$$

where $1 \leq j \leq N_{x}, 1 \leq m \leq N_{y}, n \geq 0$

By Taylor's expansion, and suppposing that $\Psi$ is smooth enough:

$$
\begin{gathered}
\frac{1}{k}\left(\Psi_{j, m}^{n+1}-\Psi_{j, m}^{n}\right)=\left(\partial_{t} \Psi\right)_{j, m}^{n}+\frac{k}{2}\left(\partial_{t}^{2} \Psi\right)_{j, m}^{n}+\frac{k^{2}}{6}\left(\partial_{t}^{3} \Psi\right)_{j, m}^{n}+\mathcal{O}\left(k^{3}\right), \\
\frac{1}{2}\left(\Psi_{j, m}^{n+1}+\Psi_{j, m}^{n}\right)=\Psi_{j, m}^{n}+\frac{k}{2}\left(\partial_{t} \Psi\right)_{j, m}^{n}+\frac{k^{2}}{4}\left(\partial_{t}^{2} \Psi\right)_{j, m}^{n}+\mathcal{O}\left(k^{3}\right), \\
\frac{1}{4 h_{x}^{2}}\left(\Psi_{j+1, m}^{n}-2 \Psi_{j, m}^{n}+\Psi_{j-1, m}^{n}\right)=\frac{1}{4}\left(\partial_{x}^{2} \Psi\right)_{j, m}^{n}+\frac{h_{x}^{2}}{12}\left(\partial_{x}^{4} \Psi\right)_{j, m}^{n}+\mathcal{O}\left(h_{x}^{4}\right), \\
\frac{1}{4 h_{x}^{2}}\left(\Psi_{j+1, m}^{n+1}-2 \Psi_{j, m}^{n+1}+\Psi_{j-1, m}^{n+1}\right)=\frac{1}{4}\left(\partial_{x}^{2} \Psi\right)_{j, m}^{n}+\frac{k}{4}\left(\partial_{x x t} \Psi\right)_{j, m}^{n}+ \\
+\frac{k^{2}}{8}\left(\partial_{x x t t} \Psi\right)_{j, m}^{n}+\frac{h_{x}^{2}}{12}\left(\partial_{x}^{4} \Psi\right)_{j, m}^{n}+\mathcal{O}\left(k^{3}\right)+\mathcal{O}\left(k h_{x}^{2}\right), \\
\frac{1}{4 h_{y}^{2}}\left(\Psi_{j, m+1}^{n}-2 \Psi_{j, m}^{n}+\Psi_{j, m-1}^{n}\right)=\frac{1}{4}\left(\partial_{y}^{2} \Psi\right)_{j, m}^{n}+\frac{h_{y}^{2}}{12}\left(\partial_{y}^{4} \Psi\right)_{j, m}^{n}+\mathcal{O}\left(h_{y}^{4}\right),
\end{gathered}
$$




$$
\begin{aligned}
\frac{1}{4 h_{y}^{2}}\left(\Psi_{j, m+1}^{n+1}-\right. & \left.2 \Psi_{j, m}^{n+1}+\Psi_{j, m-1}^{n+1}\right)=\frac{1}{4}\left(\partial_{y}^{2} \Psi\right)_{j, m}^{n}+\frac{k}{4}\left(\partial_{y y t} \Psi\right)_{j, m}^{n}+ \\
+ & \frac{k^{2}}{8}\left(\partial_{y y t t} \Psi\right)_{j, m}^{n}+\frac{h_{y}^{2}}{12}\left(\partial_{y}^{4} \Psi\right)_{j, m}^{n}+\mathcal{O}\left(k^{3}\right)+\mathcal{O}\left(k h_{y}^{2}\right) .
\end{aligned}
$$

Finally

$$
\begin{aligned}
\mathcal{P}_{\tau, h} \Phi & -\mathcal{P} \Phi=\mathbf{I}_{2}\left(\partial_{t} \Psi\right)_{j, m}^{n}-\mathbf{J}_{2}\left(\frac{1}{2}\left(\triangle_{x, y} \Psi\right)_{j, m}^{n}-V_{C}(x, y) \Psi_{j, m}^{n}\right)+ \\
& +\frac{k}{2}\left(\mathbf{I}_{2} \partial_{t}-\mathbf{J}_{2}\left(\frac{1}{2} \triangle_{x, y}-V_{C}\left(x_{j}, y_{m}\right)\right)\right) \partial_{t} \Psi_{j, m}^{n}-\mathbf{I}_{2}\left(\partial_{t} \Psi\right)_{j, m}^{n}+ \\
& +\mathbf{J}_{2}\left(\frac{1}{2}\left(\triangle_{x, y} \Psi\right)_{j, m}^{n}-V_{C}\left(x_{j}, y_{m}\right) \Psi_{j, m}^{n}\right)+\mathcal{O}\left(h_{x}^{2}\right)+\mathcal{O}\left(h_{y}^{2}\right)+\mathcal{O}\left(k^{2}\right)= \\
& =\mathcal{O}\left(h_{x}^{2}\right)+\mathcal{O}\left(h_{y}^{2}\right)+\mathcal{O}\left(k^{2}\right)
\end{aligned}
$$

and then conclude that the FDS (3.71) is order 2 accurate in space $(x, y)$ and time $t$. Hence, following Propositions 3.6 and 3.7, we conclude that the overall accuracy of the numerical scheme has the $2^{\text {nd }}$ order as well.

Proposition $3.9\left(\ell^{2}\right.$-stability of the scheme (3.71)). FDS (3.71) approximating the Schrödinger equation with the constant potential $V_{C}(3.69)$ is $\ell^{2}$-stable.

Proof. To prove the stability, we use the numerical scheme in the form (3.81), rewritten as:

$$
\widetilde{A} \Psi^{n+1}=\widetilde{B} \Psi^{n}
$$

where $\widetilde{A}$ and $\widetilde{B}$ are two real heptadiagonal matrices

$$
\widetilde{A}=\left[\begin{array}{cc}
A_{R} & -I \\
I & A_{R}
\end{array}\right], \quad \widetilde{B}=\left[\begin{array}{cc}
-A_{R} & -I \\
I & -A_{R}
\end{array}\right]
$$

size of $2 N_{x} N_{y} \times 2 N_{x} N_{y}$ each, while $\Psi^{n+1}$ and $\Psi^{n}$ are vectors in $\mathbb{C}^{2 N_{x} N_{y}}$ :

$$
\Psi^{n}=\left(\psi_{R}^{n}, \psi_{I}^{n}\right)^{T}
$$


Then, we express from $(3.95)$

$$
\Psi^{n+1}=\widetilde{A}^{-1} \widetilde{B} \Psi^{n}
$$

Indeed, we can state that $\widetilde{A}$ is invertible due to invertibility of $A_{R}$ following from the linear independence of its coulomn-vectors, see Figs. 3.5 and 3.6.

Let us introduce the grid dependent $\ell^{2}$-norm on square $\left(N_{x}=N_{y}, h:=h_{x}=h_{y}\right)$ as

$$
\|\Psi\|_{h}^{2}=h \sum_{l=1}^{2 N_{x}^{2}} \Psi_{l}^{2}=h \Psi^{T} \Psi .
$$

From (3.97), we have

$$
\left(\Psi^{n+1}\right)^{T}=\left(\Psi^{n}\right)^{T} \widetilde{B}^{T}\left(\widetilde{A}^{-1}\right)^{T}
$$

and hence

$$
\left(\Psi^{n+1}\right)^{T} \Psi^{n+1}=\left(\Psi^{n}\right)^{T} \widetilde{B}^{T}\left(\widetilde{A}^{-1}\right)^{T} \widetilde{A}^{-1} \widetilde{B} \Psi^{n}=\left(\Psi^{n}\right)^{T} \widetilde{B}^{T}\left(\widetilde{A} \widetilde{A}^{T}\right)^{-1} \widetilde{B} \Psi^{n}
$$

Before we proceed with evaluation (3.99), we need to compute several matrix expressions. Namely, the transposed matrices:

$$
\widetilde{A}^{T}=\left[\begin{array}{cc}
A_{R} & I \\
-I & A_{R}
\end{array}\right], \quad \widetilde{B}^{T}=\left[\begin{array}{cc}
-A_{R} & I \\
-I & -A_{R}
\end{array}\right]
$$

and their multiplication:

$$
G:=\widetilde{A}^{T} \widetilde{A}=\widetilde{A} \widetilde{A}^{T}=\widetilde{B}^{T} \widetilde{B}=\widetilde{B} \widetilde{B}^{T}=\left[\begin{array}{cc}
A_{R}^{2}+I & 0 \\
0 & A_{R}^{2}+I
\end{array}\right]
$$

$G$ is trivially invertible. Now, we can accomplish (3.99)

$$
\begin{gathered}
\left(\Psi^{n+1}\right)^{T} \Psi^{n+1}=\left(\Psi^{n}\right)^{T} \widetilde{B}^{T} G^{-1} \widetilde{B} \widetilde{B}^{T}\left(\widetilde{B}^{T}\right)^{-1} \Psi^{n}=\left(\Psi^{n}\right)^{T} \widetilde{B}^{T} G^{-1} G\left(\widetilde{B}^{T}\right)^{-1} \Psi^{n}= \\
=\left(\Psi^{n}\right)^{T} \widetilde{B}^{T}\left(\widetilde{B}^{T}\right)^{-1} \Psi^{n}=\left(\Psi^{n}\right)^{T} \Psi^{n}
\end{gathered}
$$


so that

$$
\left\|\Psi^{n+1}\right\|_{h}^{2}=\left\|\Psi^{n}\right\|_{h}^{2}
$$

As it was expected, the Crank-Nicolson method is unconditionally stable and conserves the $\ell^{2}$-norm of the wavefunction.

\subsubsection{Solving of the Matrix Equation}

Thus, the problem (3.69) is reduced to solving the linear system (3.95). Notice that in our computations $N_{x}=N_{y}=200-500$, i.e. typically $\widetilde{A}$ and $\widetilde{B}$ belong to $M_{80,000}(\mathbb{R})-M_{500,000}(\mathbb{R})$. As these matrices are sparse, it is reasonable to store only non-zero entries, and the Compressed Row Storage (CRS) format was used. Recall that in this format, we need only to save only 3 linear arrays, see Tab. 3.1.

\begin{tabular}{|c|l|}
\hline array & contains \\
\hline val & non-zero values of the matrix (left-to-right, then top-to bottom) \\
col_ind & column indices corresponding to the values \\
row_ptr & list of value indexes, C-pointers, where each row starts \\
\hline
\end{tabular}

TABle 3.1: Compressed Row Storage format for sparse matrixes

The CRS format is a standard storage format in numerical linear algebra. In particular, it is represented by C-library PReconditioned Iterative MultiMethod Eigensolver (PRIMME), see [110], [122], and SparseLib++ for sparse matrices, see [100], [42]. In order to solve the linear system (3.95), first, it calls the effective algorithm for multiplication of the sparse matrix $\widetilde{B}$ stored in the CRS format, and the vector $\widetilde{\Psi}^{n}$ : $\widetilde{b}=\widetilde{B} \widetilde{\Psi}^{n}$. Finally, to solve the linear system $\widetilde{A} \widetilde{\Psi}^{n+1}=\widetilde{b}$ with the sparse matrix $\widetilde{A}$, we use the Generalized Minimal Residual (GMRES) method for nonsymmetric systems, based on Krylov subspace iterations [101]. 


\subsection{Discretization of the Polarization Evolution Equation}

To compute the components of EM field at each time step $t^{n}$ according to Scheme (3.33), we need to determine $\left(\partial_{t} P_{y^{\prime}}\right)_{i}^{n},\left(\partial_{t} P_{y^{\prime}}\right)_{i}^{n+1}$ and $\left(\partial_{t} P_{y^{\prime}}\right)_{i \pm 1}^{n}$. To solve the wave equation (2.36) we use a standard method, see e.g. [101], introducing two new variables: $S=\partial_{t} P_{y^{\prime}}, R=v_{g} \partial_{z^{\prime}} P_{y^{\prime}}$, so that we can rewrite the second order evolution equation as a system of two first order advection equations. The second equation is nonlinear and reads

$$
\left\{\begin{aligned}
\partial_{t} R=v_{g} \partial_{z^{\prime}} S & \\
\partial_{t} S=a\left(E_{y^{\prime}}\right) & v_{g} \partial_{z^{\prime}} R-a\left(E_{y^{\prime}}\right) g\left(E_{y^{\prime}}\right) \partial_{t} J_{y^{\prime}}+ \\
& +a\left(E_{y^{\prime}}\right) b\left(E_{y^{\prime}}\right)\left\{\left[c \partial_{z^{\prime}} B_{x^{\prime}}-4 \pi\left(S+J_{y^{\prime}}\right)\right]^{2}-c^{2}\left(\partial_{z^{\prime}} E_{y^{\prime}}\right)^{2}\right\},
\end{aligned}\right.
$$

where the variable coefficients $a\left(E_{y^{\prime}}\left(z^{\prime}, t\right)\right), b\left(E_{y^{\prime}}\left(z^{\prime}, t\right)\right)$, and $g\left(E_{y^{\prime}}\left(z^{\prime}, t\right)\right)$ are defined according to (2.37). The system (3.101) should be solved with the initial conditions: $S\left(z^{\prime}, 0\right)=\partial_{t} P_{y^{\prime}}\left(z^{\prime}, 0\right)=0$ and $R\left(z^{\prime}, 0\right)=0$ - as long as the laser pulse has not reached the region of gas, the polarization of the medium is constant. Lastly, by solving the TDSE at the set of preselected points, we obtain the boundary conditions: $S\left(z_{\alpha, 1}^{\prime}, t\right)=\partial_{t} P_{y^{\prime}}\left(z_{\alpha, 1}^{\prime}, t\right)\left(\alpha=1,2 \ldots N_{1}\right)$.

To obtain a second order scheme in time and space, we use the Lax-Wendroff method [113]. First, we expand $R$ and $S$ in Taylor series up to $\mathcal{O}\left(\tau^{3}\right)$ and express the second order derivatives in time through spatial derivatives:

$$
\begin{aligned}
\partial_{t}^{2} R=v_{g} \partial_{z^{\prime} t} S & =v_{g}^{2} \partial_{z^{\prime}}\left(a \partial_{z^{\prime}} R\right)-v_{g} \partial_{z^{\prime}}\left(a g \partial_{t} J_{y^{\prime}}\right)+ \\
& +\partial_{z^{\prime}}\left\{a b\left[\left(c \partial_{z^{\prime}} B_{x^{\prime}}-4 \pi\left(S+J_{y^{\prime}}\right)\right)^{2}-c^{2}\left(\partial_{z^{\prime}} E_{y^{\prime}}\right)^{2}\right]\right\}
\end{aligned}
$$


where for simplicity notation we denote $\left.a:=a\left(E_{y^{\prime}}\right), b:=b\left(E_{y^{\prime}}\right), g:=g\left(E_{y^{\prime}}\right)\right)$ and the time derivative $\partial_{t} J_{y^{\prime}}$ should be taken from Drude's equation (2.5). Similarly we find

$$
\begin{aligned}
& \partial_{t}^{2} S=a v_{g}^{2} \partial_{z^{\prime}}^{2} S+\left(\partial_{t} a\right) v_{g} \partial_{z^{\prime}} R-a g \partial_{t}^{2} J_{y^{\prime}}-\partial_{t}(a g) \partial_{t} J_{y^{\prime}}+ \\
& +2 a b\left\{\left[c \partial_{z^{\prime}} B_{x^{\prime}}-4 \pi\left(S+J_{y^{\prime}}\right)\right]\left[c \partial_{z^{\prime} t} B_{x^{\prime}}-4 \pi\left(\partial_{t} S+\partial_{t} J_{y^{\prime}}\right)\right]-c^{2}\left(\partial_{z^{\prime}} E_{y^{\prime}}\right)\left(\partial_{z^{\prime} t} E_{y^{\prime}}\right)\right\}+ \\
& +\partial_{t}(a b)\left\{\left[c \partial_{z^{\prime}} B_{x^{\prime}}-4 \pi\left(S+J_{y^{\prime}}\right)\right]^{2}-c^{2}\left(\partial_{z^{\prime}} E_{y^{\prime}}\right)^{2}\right\}
\end{aligned}
$$

To reduce all time derivatives in the expression for $\partial_{t}^{2} S$ we notice that, according to MEs, $\partial_{t} E_{y^{\prime}}=c \partial_{z^{\prime}} B_{x^{\prime}}-4 \pi\left(S+J_{y^{\prime}}\right), \partial_{t} B_{x^{\prime}}=c \partial_{z^{\prime}} E_{y^{\prime}}$, so that $\partial_{z^{\prime} t} E_{y^{\prime}}=c \partial_{z^{\prime}}^{2} B_{x^{\prime}}-$ $4 \pi \partial_{z^{\prime}}\left(S+J_{y^{\prime}}\right)$ and $\partial_{z^{\prime} t} B_{x^{\prime}}=c \partial_{z^{\prime}}^{2} E_{y^{\prime}}$. In addition (see (2.37)):

$$
\begin{aligned}
\partial_{t} a & =-4 \pi a^{2} b \partial_{t} E_{y^{\prime}}=-4 \pi a^{2} b\left[c \partial_{z^{\prime}} B_{x^{\prime}}-4 \pi\left(S+J_{y^{\prime}}\right)\right], \\
\partial_{t}(a b) & =\left(-4 \pi a^{2} b^{2}+a \widetilde{b}\right) \partial_{t} E_{y^{\prime}}=\left(-4 \pi a^{2} b^{2}+a \widetilde{b}\right)\left[c \partial_{z^{\prime}} B_{x^{\prime}}-4 \pi\left(S+J_{y^{\prime}}\right)\right], \\
\partial_{t}(a g) & =-4 \pi a^{2} b\left(4 \pi\left(v_{g} / c\right)^{2} \chi^{(1)}-1\right)\left[c \partial_{z^{\prime}} B_{x^{\prime}}-4 \pi\left(S+J_{y^{\prime}}\right)\right],
\end{aligned}
$$

where the coefficient $\widetilde{b}:=d b / d E_{y^{\prime}}$ is defined as

$$
\widetilde{b}=\left(v_{g} / c\right)^{2}\left(3 ! \chi^{(3)}+\frac{5 !}{2 !} \chi^{(5)} E_{y^{\prime}}^{2}+\frac{7 !}{4 !} \chi^{(7)} E_{y^{\prime}}^{4}+\ldots\right) .
$$

Notice that $\partial_{t} S$ can be expressed through the spatial derivatives according to the second equation (3.101), while according to Drude's theory (2.5)

$$
\partial_{t} J_{y^{\prime}}=-\nu_{e} J_{y^{\prime}}+\mathcal{N}_{e} E .
$$

From the last equation and (2.29) the second derivative in time of the current density:

$$
\begin{aligned}
\partial_{t}^{2} J_{y^{\prime}} & =-\nu_{e} \partial_{t} J_{y^{\prime}}+E_{y^{\prime}} \partial_{t} \mathcal{N}_{e}+\mathcal{N}_{e} \partial_{t} E_{y^{\prime}}= \\
& =\nu_{e}^{2} J_{y^{\prime}}-\nu_{e} \mathcal{N}_{e} E_{y^{\prime}}-E_{y^{\prime}} v_{g} \partial_{z^{\prime}} \mathcal{N}_{e}+\mathcal{N}_{e}\left[c \partial_{z^{\prime}} B_{x^{\prime}}-4 \pi\left(S+J_{y^{\prime}}\right)\right]
\end{aligned}
$$


Thus, using for all spatial derivatives the second order accurate approximation, we obtain for $R$ and $S$ the following scheme (here for convenience of notation: $E_{i}^{n}:=\left(E_{y^{\prime}}\right)_{i}^{n}$, $B_{i}^{n}:=\left(B_{x^{\prime}}\right)_{i}^{n}$, and $\left.J_{i}^{n}:=\left(J_{y^{\prime}}\right)_{i}^{n}\right)$

$$
\left\{\begin{array}{c}
R_{i}^{n+1}=R_{i}^{n}+\frac{\widetilde{\lambda}}{2}\left(S_{i+1}^{n}-S_{i-1}^{n}\right)+\frac{\widetilde{\lambda}^{2}}{2}\left[a_{i+\frac{1}{2}}^{n}\left(R_{i+1}^{n}-R_{i}^{n}\right)-a_{i-\frac{1}{2}}^{n}\left(R_{i}^{n}-R_{i-1}^{n}\right)\right]+ \\
+\frac{\widetilde{\lambda} \tau}{4}\left\{\widetilde{a}_{i+1}^{n}\left[\nu_{e} J_{i+1}^{n}-\left(\mathcal{N}_{e}\right)_{i+1}^{n} E_{i+1}^{n}\right]-\widetilde{a}_{i-1}^{n}\left[\nu_{e} J_{i-1}^{n}-\left(\mathcal{N}_{e}\right)_{i-1}^{n} E_{i-1}^{n}\right]\right\}+\left(R^{N L}\right)_{i}^{n} \\
S_{i}^{n+1}=S_{i}^{n}+\frac{\widetilde{\lambda}}{2} a_{i}^{n}\left(R_{i+1}^{n}-R_{i-1}^{n}\right)+\frac{\widetilde{\lambda}^{2}}{2} a_{i}^{n}\left(S_{i+1}^{n}-2 S_{i}^{n}+S_{i-1}^{n}\right)+ \\
+\widetilde{a}_{i}^{n} \tau\left\{\left[1-\frac{\tau \nu_{e}}{2}\right]\left[\nu_{e} J_{i}^{n}-\left(\mathcal{N}_{e}\right)_{i}^{n} E_{i}^{n}\right]+2 \pi \tau\left(\mathcal{N}_{e}\right)_{i}^{n}\left(S_{i}^{n}+J_{i}^{n}\right)+\right. \\
\left.+\frac{\widetilde{\lambda}}{4} E_{i}^{n}\left[\left(\mathcal{N}_{e}\right)_{i+1}^{n}-\left(\mathcal{N}_{e}\right)_{i-1}^{n}\right]-\frac{\lambda}{4}\left(\mathcal{N}_{e}\right)_{i}^{n} \delta B_{i}^{n}\right\}+\left(S^{N L}\right)_{i}^{n},
\end{array}\right.
$$

where $\lambda=\frac{c \tau}{h}, \widetilde{\lambda}:=\frac{v_{g} \tau}{h}, \widetilde{a}:=a g=a\left(4 \pi\left(v_{g} / c\right)^{2} \chi^{(1)}-1\right)+1, \delta B_{i}^{n}:=B_{i+1}^{n}-B_{i-1}^{n}$, and $N_{2} \geq i \geq 2$ for each subdomain, see Fig. 2.7. Similar to (3.41), the coefficients $a_{i \pm \frac{1}{2}}^{n}$ are evaluated at $\left(E_{i}^{n}+E_{i \pm 1}^{n}\right) / 2$. Note that, in case of linear approximation, in expressions (3.105) for $R_{i}^{n+1}$ and $S_{i}^{n+1}$, only the non-vanishing terms are explicitly presented. Indeed, in the case of linear approximation $\left(\chi^{(2 m+1)}=0\right.$, for all $\left.m \geq 1\right)$, we have $a=1 / \sqrt{1+4 \pi \chi^{(1)}}$, while $b$ and $\widetilde{b}$ become zeros. However, if we want to include the possible nonlinear effects during the pulse propagation between the cells, we need to consider all the terms in (3.101)-(3.103). With this purpose we write down the expressions for the nonlinear additives in the more convenient form:

$$
\begin{array}{r}
R^{N L}=\frac{\tau^{2}}{2}\left[\partial_{z^{\prime}}(a b) U V+a b \partial_{z^{\prime}}(U V)\right]=\tau^{2}\left(-2 \pi a^{2} b^{2}+\frac{a \widetilde{b}}{2}\right)\left(\partial_{z^{\prime}} E_{y^{\prime}}\right) U V+ \\
+\tau^{2} a b T\left[c \partial_{z^{\prime}}^{2} B_{x^{\prime}}-4 \pi \partial_{z^{\prime}}\left(S+J_{y^{\prime}}\right)\right]-\tau^{2} a b c^{2}\left(\partial_{z^{\prime}}^{2} E_{y^{\prime}}\right)\left(\partial_{z^{\prime}} E_{z^{\prime}}\right),
\end{array}
$$

where three intermediary functions were introduced: $T:=c \partial_{z^{\prime}} B_{x^{\prime}}-4 \pi\left(S+J_{y^{\prime}}\right)$, $U:=T-c \partial_{z^{\prime}} E_{y^{\prime}}$ and $V:=T+c \partial_{z^{\prime}} E_{y^{\prime}}$. Similarly we get for another variable: 


$$
\begin{aligned}
S^{N L}= & \tau a b U V+\frac{\tau^{2}}{2}\left\{\left(-4 \pi a^{2} b\right) T v_{g} \partial_{z^{\prime}} R+\left(-4 \pi a^{2} b^{2}+a \widetilde{b}\right) T U V+\right. \\
& +2 a b\left[c^{2} T \partial_{z^{\prime}}^{2} E_{y^{\prime}}-4 \pi T\left(a v_{g} \partial_{z^{\prime}} R+(1-\widetilde{a})\left(-\nu_{e} J_{y^{\prime}}+\mathcal{N}_{e} E_{y^{\prime}}\right)+a b U V\right)-\right. \\
& \left.\left.-c^{2} \partial_{z^{\prime}} E_{y^{\prime}} \partial_{z^{\prime}} T\right]+4 \pi a^{2} b T\left(4 \pi\left(v_{g} / c\right)^{2} \chi^{(1)}-1\right)\left(-\nu_{e} J_{y^{\prime}}+\mathcal{N}_{e} E_{y^{\prime}}\right)\right\}= \\
= & \tau a U V\left(b+\widetilde{b} T \frac{\tau}{2}\right)+\tau^{2} a b c^{2}\left(T \partial_{z^{\prime}}^{2} E_{y^{\prime}}-\partial_{z^{\prime}} E_{y^{\prime}}\left[c \partial_{z^{\prime}}^{2} B_{x^{\prime}}-4 \pi \partial_{z^{\prime}}\left(S+J_{y^{\prime}}\right)\right]\right)- \\
& -6 \tau^{2} \pi a^{2} b T\left[v_{g} \partial_{z^{\prime}} R+b U V+\left(4 \pi\left(v_{g} / c\right)^{2} \chi^{(1)}-1\right)\left(\nu_{e} J_{y^{\prime}}-\mathcal{N}_{e} E_{y^{\prime}}\right)\right],
\end{aligned}
$$

From here the scheme in the nonlinear terms reads

$$
\left\{\begin{array}{c}
\left(R^{N L}\right)_{i}^{n}=\frac{\tau \lambda}{4 c}\left(-4 \pi\left(a_{i}^{n}\right)^{2}\left(b_{i}^{n}\right)^{2}+a_{i}^{n} \widetilde{b}_{i}^{n}\right) \delta E_{i}^{n} U_{i}^{n} V_{i}^{n}+ \\
+\frac{\lambda}{c} a_{i}^{n} b_{i}^{n}\left[\lambda T_{i}^{n} \delta^{2} B_{i}^{n}-2 \pi \tau T_{i}^{n}\left(S_{i+1}-S_{i-1}+\delta J_{i}^{n}\right)-\frac{\lambda^{2}}{2 \tau} \delta E_{i}^{n} \delta^{2} E_{i}^{n}\right], \\
\left(S^{N L}\right)_{i}^{n}=\tau a_{i}^{n} U_{i}^{n} V_{i}^{n}\left(b_{i}^{n}+\widetilde{b}_{i}^{n} T_{i}^{n} \frac{\tau}{2}\right)+a_{i}^{n} b_{i}^{n} \lambda^{2}\left\{T_{i}^{n} \delta^{2} E_{i}^{n}-\delta E_{i}^{n}\left[\frac{\lambda}{2 \tau} \delta^{2} B_{i}^{n}-\right.\right. \\
\left.-\pi\left(S_{i+1}^{n}-S_{i-1}^{n}+\delta J_{i}^{n}\right]\right\}-6 \pi \tau\left(a_{i}^{n}\right)^{2} b_{i}^{n} T_{i}^{n}\left[\frac{\tilde{\lambda}}{2}\left(R_{i+1}^{n}-R_{i-1}^{n}\right)+\tau b_{i}^{n} U_{i}^{n} V_{i}^{n}+\right. \\
\left.+\tau\left(4 \pi\left(v_{g} / c\right)^{2} \chi^{(1)}-1\right)\left(\nu_{e} J_{i}^{n}-\left(\mathcal{N}_{e}\right)_{i}^{n} E_{i}^{n}\right)\right],
\end{array}\right.
$$

where for brevity's sake it is denoted: $\delta E_{i}^{n}=E_{i+1}^{n}-E_{i-1}^{n}, \delta J_{i}^{n}=J_{i+1}^{n}-J_{i-1}^{n}$, $\delta^{2} E_{i}^{n}=E_{i+1}^{n}-2 E_{i}^{n}+E_{i-1}^{n}, \delta^{2} B_{i}^{n}=B_{i+1}^{n}-2 B_{i}^{n}+B_{i-1}^{n}, T_{i}^{n}=\frac{\lambda}{2 \tau} \delta B_{i}^{n}-4 \pi\left(S_{i}^{n}+J_{i}^{n}\right)$, $U_{i}^{n}=T_{i}^{n}-\frac{\lambda}{2 \tau} \delta E_{i}^{n}$, and $V_{i}^{n}=T_{i}^{n}+\frac{\lambda}{2 \tau} \delta E_{i}^{n}$.

Recall, the updated values of $R$ and $S$ at the set of boundary points $z_{\alpha}^{\prime}=$ $\left\{z_{1}^{\prime}, z_{2}^{\prime}, \ldots, z_{N_{1}}\right\}$ are obtained from solving TDSEs:

$$
\left\{\begin{array}{l}
S_{\alpha, 1}^{n+1}=\left(\partial_{t} P_{y^{\prime}}\right)_{\alpha, 1}^{n+1}, \quad R_{\alpha, 1}^{n+1}=-S_{\alpha, 1}^{n+1}, \quad 1 \geq \alpha \geq N_{1} \\
S_{\alpha, N_{2}+1}^{n+1}=\left(\partial_{t} P_{y^{\prime}}\right)_{\alpha, N_{2}+1}^{n+1}, \quad R_{\alpha, N_{2}+1}^{n+1}=-S_{\alpha, N_{2}+1}^{n+1}, \quad 1 \geq \alpha \geq N_{1} \\
S_{N_{1}, N_{2}}^{n+1}=0, R_{N_{1}, N_{2}}^{n+1}=0 .
\end{array}\right.
$$

Obviously that $R_{\alpha, N_{2}+1}=R_{\alpha+1,1}$ and $S_{\alpha, N_{2}+1}=S_{\alpha+1,1}$ for $\alpha<N_{1}$. Thus, for each subdomain we use outgoing boundary conditions for the wave.

It is difficult to provide the general stability analysis for the scheme represented by (3.105) with nonlinear terms given by (3.106). However, in Section 4 we study the 
convergence of this scheme numerically.

Now we are ready to implement the procedure described on Fig. 2.7 for the case of $1 \mathrm{~d}-2 \mathrm{~d}$ MASP model enriched by $1 \mathrm{~d}$ polarization evolution equation. To simulate 1d-propagation of the time derivative of polarization vector, we use numerical scheme (3.105), (3.106) with BCs (3.107).

\subsection{Integrating of Drude's Equation}

To preserve the second order accuracy of the overall numerical model, we need to use at least a second order scheme in time to integrate the equation for the current of free electrons (2.5). It seems too "expensive" to solve the MEs and the current equation together as a system (at least, assuming the second order in time), so we split the problem into 2 steps: (i) updating the current of electrons according to known fields, and (ii) computing the EM field with a given current. In addition, in order to not increase the computational complexity, we would like to avoid recalculations of Maxwell's equations in the intermediate time points (i.e. twice within one computational cycle). With all these conditions in mind, we choose for our purposes Adams-Bashforth's $2^{\text {nd }}$ order consistent method that employs integration via interpolation [101]:

$$
\mathbf{J}_{i}^{n+1}=\mathbf{J}_{i}^{n}+\frac{\Delta t}{2}\left\{3\left[-\nu_{e} \mathbf{J}_{i}^{n}+\left(\mathcal{N}_{e}\right)_{i}^{n} \mathbf{E}_{i}^{n}\right]-\left[-\nu_{e} \mathbf{J}_{i}^{n-1}+\left(\mathcal{N}_{e}\right)_{i}^{n-1} \mathbf{E}_{i}^{n-1}\right]\right\} .
$$

Notice that to launch this 2-step scheme, we need two sets of initial data: $\mathbf{J}_{i}^{0}$ and $\mathbf{J}_{i}^{1}$ $(N \geq i \geq 0)$, while the initial data provides only one value, $\mathbf{J}_{i}^{0}=0$. The common solution of this problem is to use a different method on the first step, e.g. one of Runge-Kutta's methods that uses values of the variables at $t^{n+1}, t^{n}$ and intermediate time points but not at $t^{n-1}[101]$. However, since in our computations we preliminary 
neglect the collisions, i.e. $\nu_{e}=0$, the scheme reduces to

$$
\mathbf{J}_{i}^{n+1}=\mathbf{J}_{i}^{n}+\frac{\Delta t}{2}\left[3\left(\mathcal{N}_{e}\right)_{i}^{n} \mathbf{E}_{i}^{n}-\left(\mathcal{N}_{e}\right)_{i}^{n-1} \mathbf{E}_{i}^{n-1}\right]
$$

requiring the initial conditions $\mathbf{J}_{i}^{0}$, only. In addition, supposing that $\mathcal{N}_{e}\left(z^{\prime}, 0\right)=0$, i.e. there is no free electron in the region before the pulse reaches it (and, moreover, for intensities under consideration, this value is not expected to rise fast), we set in (3.109) $\left(\mathcal{N}_{e}\right)_{i}^{0}=0$ and $\left(\mathcal{N}_{e}\right)_{i}^{-1}=0$, to launch the scheme while avoiding increased complexity.

Now we show that the local truncation error of the 2-step method (3.108) has the order 2. Indeed, from Taylor's expansion (for convenience of notation we omit here the spatial index $i)$ :

$$
\begin{array}{r}
\mathbf{J}^{n \pm 1}=\mathbf{J}^{n} \pm\left(\partial_{t} \mathbf{J}\right)^{n} \Delta t+\left(\partial_{t}^{2} \mathbf{J}\right)^{n} \frac{(\Delta t)^{2}}{2}+\mathcal{O}\left((\Delta t)^{3}\right)=\mathbf{J}^{n} \pm\left[-\nu_{e} \mathbf{J}^{n}+\mathcal{N}_{e}^{n} \mathbf{E}^{n}\right] \Delta t+ \\
+\left[\nu_{e}^{2} \mathbf{J}^{n}-\nu_{e} \mathcal{N}_{e}^{n} \mathbf{E}^{n}+\frac{\mathcal{N}_{e}^{n} \mathbf{E}^{n}-\mathcal{N}_{e}^{n-1} \mathbf{E}^{n-1}}{\Delta t}\right] \frac{(\Delta t)^{2}}{2}+\mathcal{O}\left((\Delta t)^{3}\right) .
\end{array}
$$

Substituting (3.110) into (3.108) we can confirm that the order of consistency of the scheme is 2:

$$
\frac{\mathbf{J}_{i}^{n+1}-\mathbf{J}_{i}^{n}}{\Delta t}+\nu_{e} \frac{3 \mathbf{J}_{i}^{n}-\mathbf{J}_{i}^{n-1}}{2}-\frac{3\left(\mathcal{N}_{e}\right)_{i}^{n} \mathbf{E}_{i}^{n}-\left(\mathcal{N}_{e}\right)_{i}^{n-1} \mathbf{E}_{i}^{n-1}}{2}=\mathcal{O}\left((\Delta t)^{2}\right)
$$

However, as a payment for 2 nd order accuracy, at each $n+1$ step we need to store not only the set $\left(\mathcal{N}_{e}\right)_{i}^{n}, \mathbf{E}_{i}^{n}, \mathbf{J}_{i}^{n}$, but also $\left(\mathcal{N}_{e}\right)_{i}^{n-1}, \mathbf{E}_{i}^{n-1}, \mathbf{J}_{i}^{n-1}$. 


\subsection{Parallel Computing Aspects}

Using parallel computing is essential for implementation of the numerical schemes described in Sections 3.1-3.5, see e.g. [76,79]. In this section, we consider several implementation of the MS and MASP models.

\subsubsection{Parallelism in the Case of 1d-2d MS Model}

Fig. 3.9 illustrates the execution in the case of the Maxwell-Schrödinger model and assuming for the sake of simplicity that the gas region consists of 4 Maxwell's cells only: $\Omega=\cup_{i=1}^{4} \Omega_{i}$. Recall that each $\Omega_{i}(i=1, \ldots, 4)$ contains a subdomain $\omega_{i}$, where the corresponding TDSE is solved. Now we describe one cycle of the computations, i.e. $t \in\left[t^{n}, t^{n+1}\right)$, see Fig. 2.1. At time $t^{n}$, the root processor and 3 slave processors compute the TDSEs at each cell of the domain $\Omega$, i.e. in case of $1 \mathrm{~d}$ MEs at the nodes $z_{i}^{\prime}$, and evaluate the polarization vectors $\mathbf{P}_{i}^{n}$ with their first derivatives in time $\left(\partial_{t} \mathbf{P}\right)_{i}^{n}$

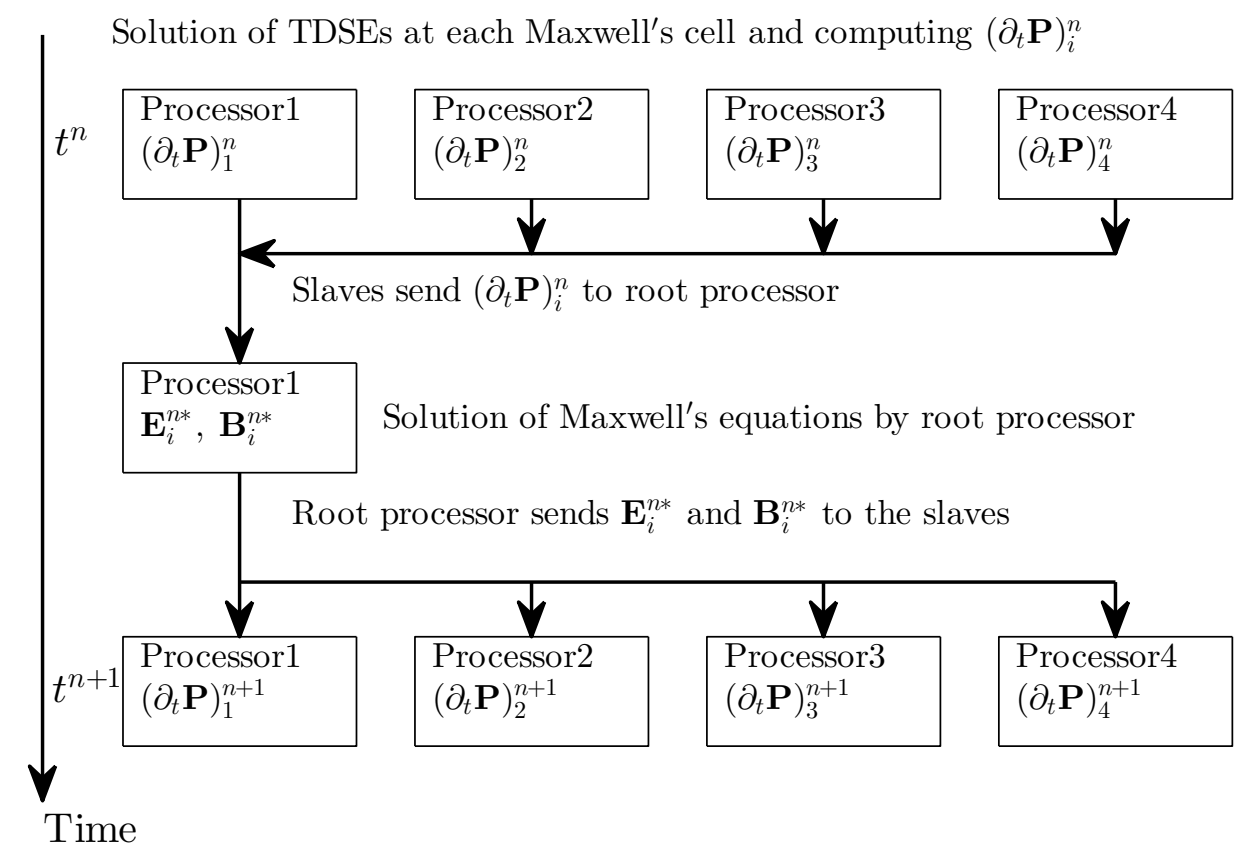

Figure 3.9: Example of parallelization: 1d-2d MS model. Index of the Maxwell's cell $i=1 \ldots N$ (here $N=4$ ). Length of the gas region $L_{g}=4 \times \Delta z^{\prime}$. 
according to $(2.2)$. Then the slave processors send these data to the root processor that updates the electric and magnetic fields, $\mathbf{E}_{i}^{n *}, \mathbf{B}_{i}^{n *}$, at time $t^{n *} \in\left(t^{n}, t^{n+1}\right)$, according to the new values of $\left(\partial_{t} \mathbf{P}\right)_{i}^{n}$, see (2.1). Then the root processor sends the updated electric field to the slave processors, and the next, $(n+1)^{\text {th }}$, cycle starts.

Remark 1. If the laser field is strong enough, the propagation length is long enough or the gas is dense enough, such that the level of ionization is high, we need to consider the dynamics of free electrons using the MASP model instead of the MS model. Then at each time step, the processors 1-4 compute and send to the root processor not only the polarization vectors, but also the free electron densities $\left(\mathcal{N}_{e}\right)_{i}^{n}$, see (2.9) and (2.10). After receiving this data, the root processor calculates from the Drude equation (2.5) the current density vector $\mathbf{J}_{i}^{n *}$, at $t^{n *} \in\left(t^{n}, t^{n * *}\right)$ in order to use it while solving the MEs at $t^{n * *}, t^{n * *} \in\left(t^{n *}, t^{n+1}\right)$, see also Section 3.6.2.

Remark 2. In the case we deal with the gas region consisting of $m$ Maxwell's cells, we can either use a higher number of slave processors $(m-1)$ or distribute the computation of the $m$ TDSEs equally between $l(l<m)$ processors (one root and $l-1$ slaves) to solve them sequentially.

\subsubsection{Parallelism in the Case of 1d-2d Model Enriched by the Polarization Evolution Equation}

We discuss here the coupling of the schemes approximating (i) the MASP model, (ii) the polarization evolution equation (2.26), and (iii) the free electron density evolution equation (2.29). As an example, we consider the case where the length of a gas region $L_{g}$, is divided in 4 subdomains each containing $N_{2}$ Maxwell's cells, see Fig. 2.7. Unlike Section 3.6.1, at time $t^{n}$ each of 4 processors solves the TDSE only in the first cell of the subdomain assigned to it, i.e. at nodes $z_{\alpha, 1}^{\prime}$, where $\alpha=\{1,2,3,4\}$ is the ordinal number of the subdomain and the second index corresponds to the position number within the subdomain, see Fig 3.10. Then data $\left(\partial_{t} \mathbf{P}\right)_{\alpha, 1}^{n}$ and $\left(\mathcal{N}_{e}\right)_{\alpha, 1}^{n}$ are sent to the 
Time Computation of TDSEs at the first cell of each subdomain with index $\alpha$, evaluating polarization and free electron density

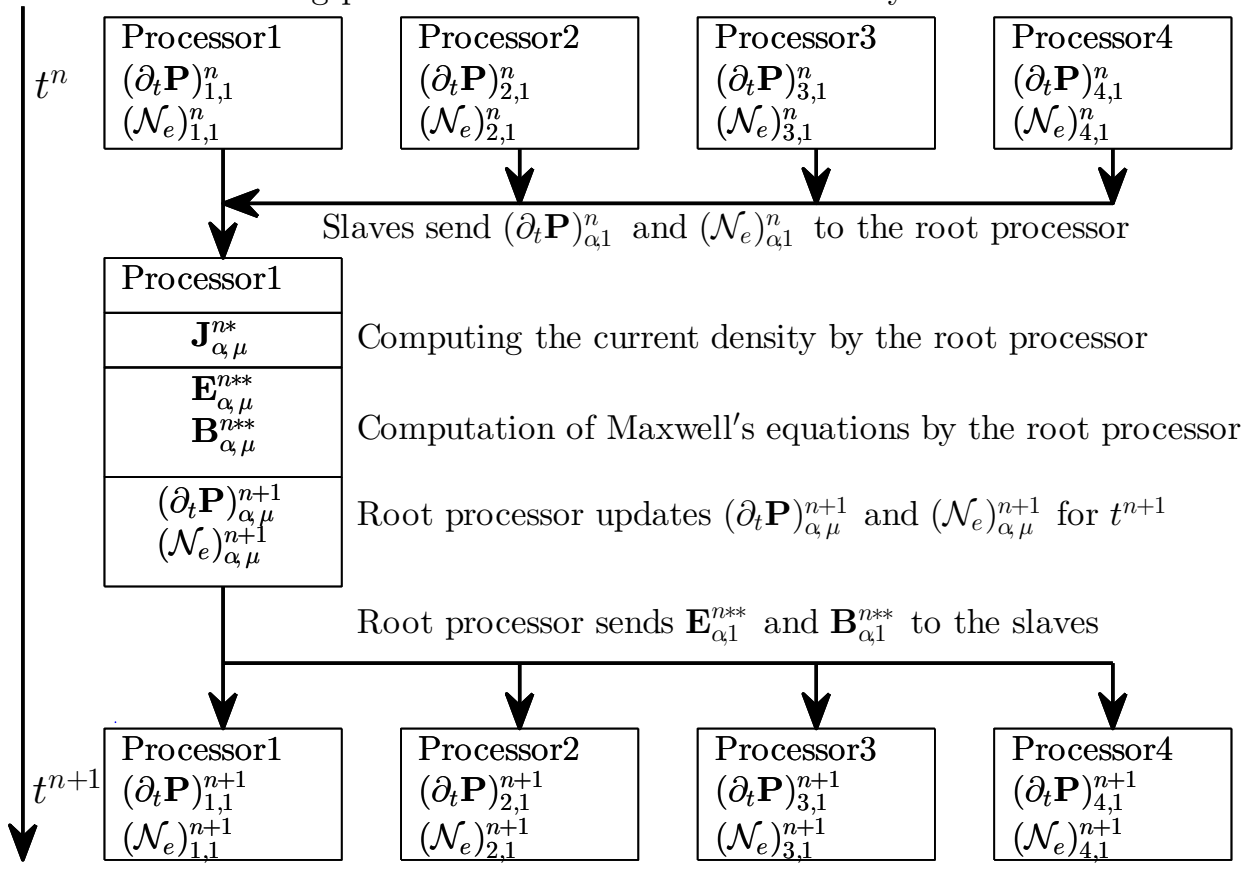

Figure 3.10: Example of parallelization: 1d-2d MASP model enriched by the evolution equations. Index of subdomain $\alpha=1 \ldots N_{1}$ (here $N_{1}=4$ ), index of the cell in the subdomain $\mu=1 \ldots N_{2}$. Length of the gas region $L_{g}=4 \times N_{2} \times \Delta z^{\prime}$.

root processor in order to update (i) the current of free electrons $\mathbf{J}_{\alpha, \mu}^{n *}$, and then (ii) the EM field at time $t^{n * *}: \mathbf{E}_{\alpha, \mu}^{n * *}, \mathbf{B}_{\alpha, \mu}^{n * *}, \mu=1, \ldots, N_{2}$. Note that at this point, we assume that the root processor has already known the values of $\left(\partial_{t} \mathbf{P}\right)_{\alpha, \mu}^{n}$ and $\left(\mathcal{N}_{e}\right)_{\alpha, \mu}^{n}$ for all spatial points $z^{\prime}{ }_{\alpha, \mu}\left(\alpha=1, \ldots, 4, \mu=1, \ldots, N_{2}\right)$, but not only for the first cells $\left\{z_{\alpha, 1}^{\prime}\right\}$ where the TDSEs are solved. To clarify that apparent contradiction, we point out that at the time $t \in\left(t^{n * *}, t^{n+1}\right)$, the root processor evaluates $\left(\partial_{t} \mathbf{P}\right)_{\alpha, \mu}^{n+1}$ and $\left(\mathcal{N}_{e}\right)_{\alpha, \mu}^{n+1}$ for the next time cycle, in all Maxwell's cells using the evolution equations for the polarization and the free electron density. Finally, similarly as was done in Section 3.6.1, we generalize the described scheme on the case of $m$ subdomains and $l$ processors, requiring the ratio $\frac{m}{l}$ to be an integer. 


\subsubsection{Parallelism for $3 \mathrm{~d}-1 \mathrm{~d} / 2 \mathrm{~d}$ Model}

We now present the computational geometry, see Fig. 3.11. As before, the direction of propagation of the pulse coincides with the axis $z^{\prime}$, while the $\mathrm{H}_{2}^{+}$-molecules are oriented in the $x y$-plane, for definiteness let it be the $y$-direction. The $3 \mathrm{~d}$ computational domain of the MEs $\Omega$, is decomposed into $N_{x^{\prime}} \times N_{y^{\prime}} \times N_{z^{\prime}}$ blocks $\Omega_{i}$ with edges $h_{\perp}=\Delta x^{\prime}=\Delta y^{\prime}$ and $h_{\|}=\Delta z^{\prime}$. The $1 \mathrm{~d}-/ 2 \mathrm{~d}-/ 3 \mathrm{~d}$-domain of the TDSE $\omega_{i}$, is included in the corresponding cell $\Omega_{i}$, such that the plane $x y$ is parallel to the plane $x^{\prime} y^{\prime}$.

We proceed to the description of one cycle of the computations, see Fig. 3.12. The domain of the MEs is divided along the $z^{\prime}$-direction into 3 regions: vacuum, gas, vacuum, while the gas region in its turn is divided into $N_{1}$ subdomains, so that each transversal layer corresponding to node $z_{\alpha, \mu}^{\prime}\left(\alpha=1, \ldots, N_{1}, \mu=1, \ldots, N_{2}\right)$ contains $N_{x^{\prime}} \times N_{y^{\prime}}$ Maxwell's cells. At time $t^{n}$, the processors indicated as P4-P8 solve $m=N_{x^{\prime}} \times N_{y^{\prime}} \times N_{1}$ TDSEs belonging to the first layers of all the $N_{1}$ subdomains.

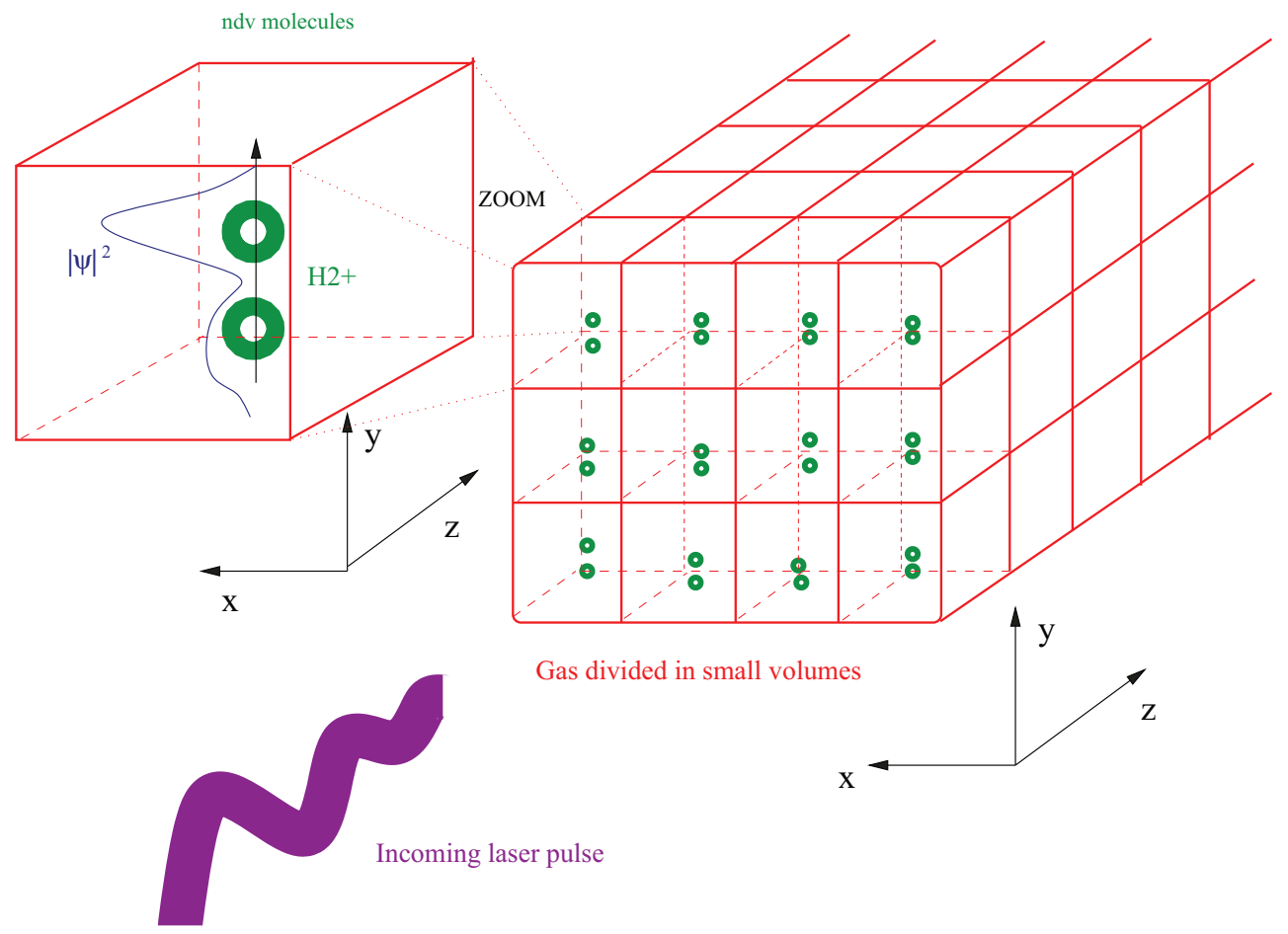

Figure 3.11: Computational geometry of 3d-(1d/2d/3d) MASP model [81]. 
Now, having the set of the wave functions, five processors P4-P8 compute the first time derivatives of the polarization and free electron number densities in each of $N_{x^{\prime}} \times N_{y^{\prime}}$ cells of these first layers. Then these data are transferred to processor P2 that computes the current of free electrons having $\left(\mathcal{N}_{e}\right)_{\alpha, \mu, \nu}^{n}\left(\nu=1, \ldots, N_{x^{\prime}} \times N_{y^{\prime}}\right)$ available for the entire gas region. This is possible, as processor P2 updated values of $\left(\mathcal{N}_{e}\right)_{\alpha, \mu, \nu}^{n}$ and $\left(\partial_{t} \mathbf{P}\right)_{\alpha, \mu, \nu}^{n}$ in the previous cycle, the same way as it explained in Section 3.6.2. After that the vector fields $\mathbf{E}_{\alpha, \mu, \nu}^{n *}$ and $\mathbf{B}_{\alpha, \mu, \nu}^{n *}$ can be computed. The last problem is solved by 3 processors: P2 treats the gas at the macroscopic level, while P1 and P2 solve the MEs in the vacuum regions. Finally, P2 solves the evolution equations to update polarization and free electrons number density for the next cycle.

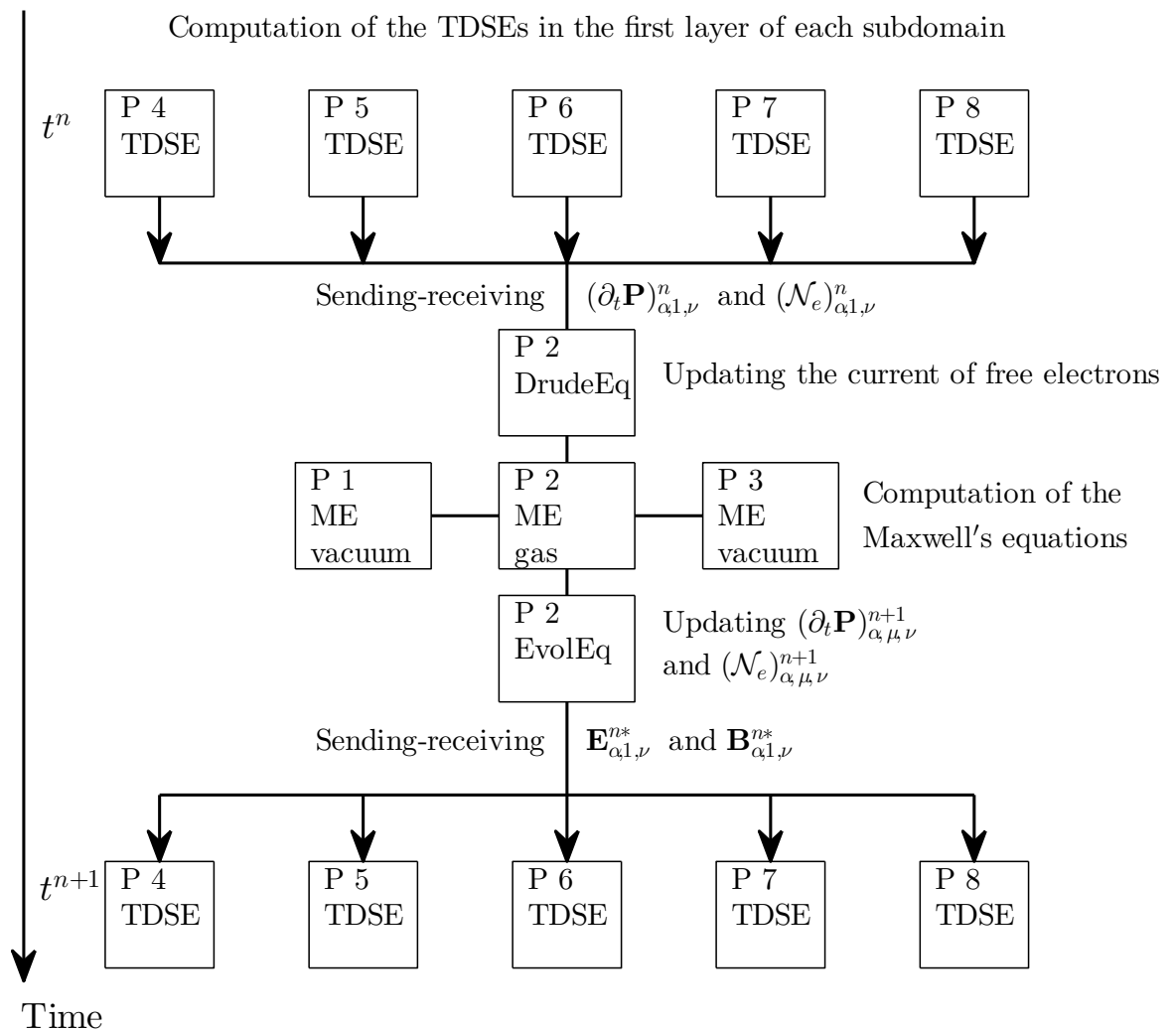

Figure 3.12: Example of parallelization: $3 \mathrm{~d}-(1 \mathrm{~d} / 2 \mathrm{~d} / 3 \mathrm{~d})$ MASP model enriched by the evolution equations. Index of the subdomain $\alpha=1 \ldots N_{1}$ (here $N_{1}=5$ ), index of the layer in the subdomain $\mu=1 \ldots N_{2}$, index of the block in the layer $\nu=1 \ldots N_{x^{\prime}} \times N_{y^{\prime}}$. Length of the gas region $L_{g}=5 \times N_{2} \times \Delta z^{\prime}$. 


\section{Chapter 4}

\section{Applications of the MS and MASP Models}

In this chapter, we show and discuss some results obtained within the MS and MASP models for linearly and circularly polarized pulses. Section 4.1 represent some results from the article [85] by Lytova, Lorin and Bandrauk (2016).

\subsection{1d-2d MS Model}

\subsubsection{Introductory Remarks and Motivation}

We study some nonlinear effects appearing during the propagation of intense and short circularly polarized $(\mathrm{CP})$ pulses in a $\mathrm{H}_{2}^{+}$-molecule gas. We simulate the evolution of the intensity and the phase of the generated odd time harmonics during the laser-molecule interaction, as a function of the propagation length of the pulse in the gas. This is possible using the Maxwell-Schrödinger (MS) propagation model presented in $[78,79,82,84]$. Recall, that the MS model equations consist of the coupling of i) macroscopic MEs modeling laser-pulses interacting with ii) many laser-molecule TDSEs from which, the macroscopic polarization from the total laser induced molec- 
ular dipole moments, are deduced using a trace operator, see Section 2.2.

High harmonic generation (HHG), see Subsection 1.4.1, in atomic/molecular gases by high intensity ultrashort linearly polarized (LP) laser pulses is currently the main method for producing coherent extreme ultraviolet and attosecond (asec) pulses [36]. This is based on a universal model of electron-recollision (see Fig. 1.9) with a maximum harmonic energy, see (1.95),

$$
N_{\max } \omega_{0}=I_{p}+3.17 U_{p}
$$

where $I_{p}$ is the ionization potential of the atom/molecule, $U_{p}=E^{2} / 4 \omega_{0}^{2}$ is the ponderomotive energy of the electron (1.93) in an oscillatory field $E(t)$ of maximum intensity $I=E^{2} / 8 \pi$ and frequency $\omega_{0}[7,10,35,39,66,72,73]$. Molecules offer an interesting medium as both ionization and recombination steps are dependent on the particular symmetry of the highest occupied molecular orbital (HOMO) and orientation $[7,66,73,118]$. Furthermore, at large distances, stretched or dissociated molecules offer the possibility of obtaining harmonics well beyond the $3.17 U_{p}$ cut-off law $(4.1),[7,15,67]$. The recombination LP-model allows to perform a full tomographic reconstruction of the $\mathrm{HOMO}$ at a high degree of spatial alignment of the molecules $[52,57,120]$. For LP-pulses the mathematical steps in structural retrieval from HHG are based upon the Strong Field Approximation (SFA), a Single Active Electron (SAE) model, and a three step process, see Fig. 1.9, [35,73]. This simple three step model can be shown to always produce a maximum return energy predicted by (4.1) even with nonzero initial velocity upon ionization [10]. Several important questions still remain open, such as the influence of the intense laser field upon the bound electronic states upon recombination [9], depletion of the initial ground state [29], the influence of the Coulomb potential on the continuum electron states [129], all effects neglected in SFA. Finally, it is important to consider macroscopic propagation 
effects as these lead to interesting new phenomena such as filamentation, see Section 1.4.2, $[97,116]$ with the conclusion that ionization dynamics can strongly influence the synthesis of isolated attosecond pulses [46]. In this study we focus on a single electron system under the Born-Oppenheimer approximation. For this $\mathrm{H}_{2}^{+}$-system, a previous TDSE simulation with exact non-Born-Oppenheimer solutions lead to enhanced ionization and HHG in the presence of an asec XUV and IR fsec pulse with the resulting efficient generation of new asec pulses [14]. A first Maxwell-Schrödinger equation for this system was then developed, based on a Slowly Varying Envelope Approximation (SVEA), leading to a first-order partial differential equation [96]. Such an approach neglected ground state depletion due to ionization, neglected backward propagation and was therefore appropriate only for low field strengths. It was nevertheless found that initial asec pulses could be shortened further in time through the resultant HHG asec pulses produced nonlinearly in the presence of an intense IR fsec pulse [11]. Later a Maxwell-TDSE model was developed in [9], where aligned $1 \mathrm{~d} \mathrm{H}_{2}^{+}$-molecules were subject to intense short LP-fields. The present consideration is an extension of [9] in the case of CP-pulses.

For circularly polarized fields interacting with atoms, recollision is predicted from classical models to be absent and require pairs of co- or counter rotating circular pulses $[13,74,131]$. We show that including propagation effects can produce low order harmonics with single CP-intense pulses in molecular systems.

We address the problem of asec pulse generation and propagation by HHG in an aligned molecular medium subject to CP-pulses $[56,89,95]$. The molecules are assumed to be aligned, and with all the same orientation, thanks to a first low intensity pulse. We emphasize that this is a not a constraint of the model and random orientation of the molecules could also be considered. The propagation effects on the generated harmonic intensities were specifically analyzed, such as the coherent generation of high energy photons as a function of the pulse propagation length. 
We conclude the introductory remarks by a discussion about the expectation of the generated harmonic intensity, as a function of propagation length. In the LPcase, it is theoretically established by perturbation theory (which, however, limits the range of validity) [24] that the harmonic intensities scale quadratically as a function of the propagation distance due to cooperative phase matching, see Subsection 1.3.1 and especially (1.67). We establish based on numerical experiments, the low-order odd-harmonic intensities scale quadratically with distance, when a CP-probe pulse propagates in a $\mathrm{H}_{2}^{+}$molecule gas (centrosymmetric medium) and that the generated photons exhibit in the HHG process circular polarization.

\subsubsection{Geometry of the Model and Calculation Parameters.}

For general description of the model, see Section 2.2. The model adaptation for $1 \mathrm{~d}-2 \mathrm{~d}$ case is given in Subsection 2.2.1: "CP-wave envelope: 1d-2d MASP model", where one also can find geometry of the numerical experiment, see Fig. 2.5, as well as ICs for the laser pulse, see (2.11) and Fig. 2.3. In such a geometry the molecular density in the second medium region (of length $L$ ) can be considered as a function of the $z^{\prime}$-coordinate only. Namely in the current computations, two options were used: a) homogeneous density (HD): $\mathcal{N}\left(z^{\prime}\right)=\mathcal{N}_{0}$, and b) non-homogeneous:

$$
\mathcal{N}\left(z^{\prime}\right)=\left\{\begin{array}{l}
\frac{\left(z^{\prime}-L_{1}\right)}{L} \frac{4 \mathcal{N}_{0}}{1-\beta^{2}}, \quad z^{\prime} \in\left[L_{1}, L_{1}+L^{\prime}\right], \\
\frac{2 \mathcal{N}_{0}}{1+\beta}, \quad z^{\prime} \in\left[L_{1}+L^{\prime}, L_{1}+L-L^{\prime}\right], \\
\frac{\left(L_{1}+L-z^{\prime}\right)}{L} \frac{4 \mathcal{N}_{0}}{1-\beta^{2}}, \quad z^{\prime} \in\left[L_{1}+L-L^{\prime}, L_{1}+L\right],
\end{array}\right.
$$



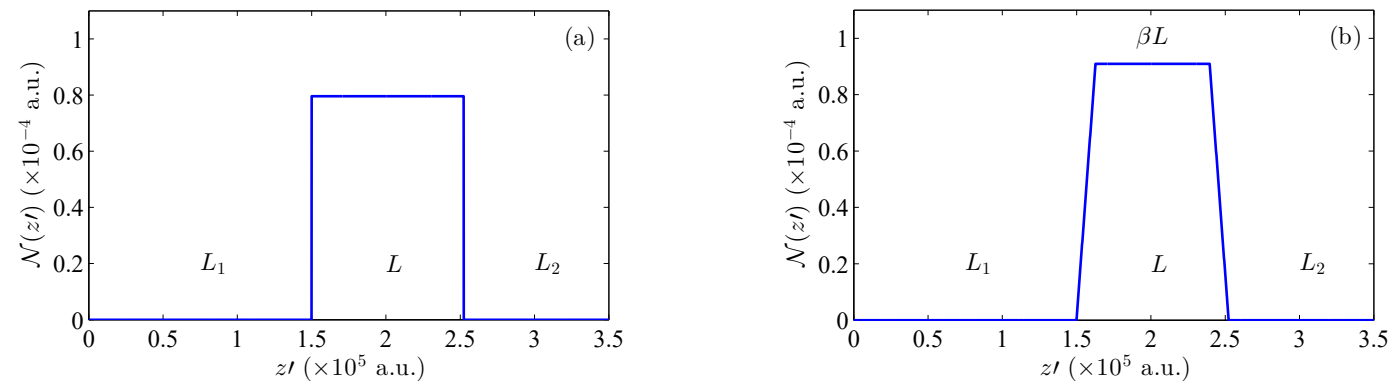

Figure 4.1: Homogeneous (a) and non-homogeneous (b) density profiles, $\mathcal{N}\left(z^{\prime}\right) ; \mathcal{N}_{0}=$ $8 \times 10^{-5} a_{0}^{-3}$. (C) 2016 American Physical Society
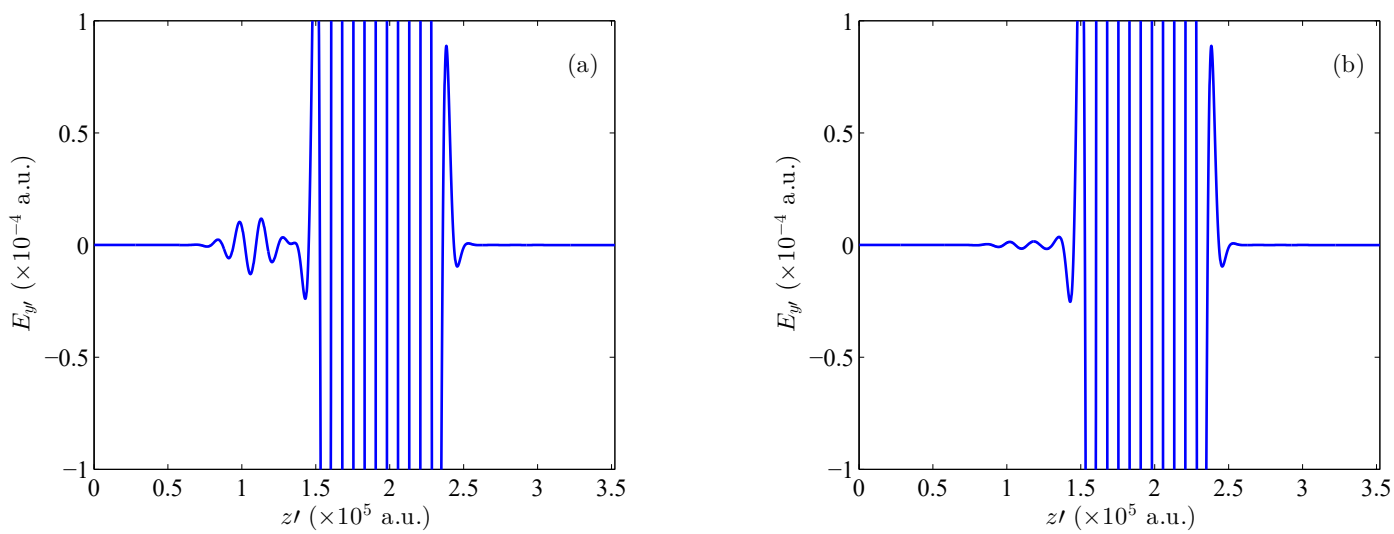

Figure 4.2: (a) Reflection of $E_{y}$-component at the entering border "vacuum-gas", $t=722$ a.u., homogeneous case. (b) Reflection of $E_{y}$-component at the entering border "vacuumgas", $t=722$ a.u., non-homogeneous case with $\beta=3 / 4$, time(a.u.) $=24$ asec. (c) 2016 American Physical Society

chosen such that $\int_{L_{1}}^{L_{1}+L} \mathcal{N}\left(z^{\prime}\right) d z^{\prime}=\mathcal{N}_{0} L$, with $L^{\prime}=\frac{1-\beta}{2} L$, where $\beta$ is a positive coefficient less than 1, see Fig 4.1.

The non-homogeneous model is found to reduce or smooth out the EM-wave reflections at both interfaces between the gas and vacuum regions, as illustrates Fig. 4.2 for the entering border case. In the reported further computations, we choose $\mathcal{N}_{0}=5.17 \times 10^{-5} a_{0}^{-3} \approx 3.5 \times 10^{20} \mathrm{~mol} / \mathrm{cm}^{3}$, corresponding to $\approx 13$ atmospheres.

We next review the numerical approximation of the MASP model by FDS. MEs are approximated by the Lax-Friedrichs scheme, see Section 3.1.1, under the Courant, Friedrichs and Lewy (CFL) stability condition, $c \Delta t_{M} / \Delta z_{M}=0.99 \lesssim 1$ (avoiding thus 
as much as possible artificial diffusion, see Section 3.2), where $\Delta t_{M}, \Delta z_{M}$ denote the time, space steps in the numerical scheme [113]. The vacuum and gas regions have lengths $L_{1}=\Delta z_{M} N_{1}, L=\Delta z_{M} N_{g}$, and $L_{2}=\Delta z_{M} N_{2}$, with spatial discretization $\Delta z_{M}=100$ a.u. $(\approx 5.29 \mathrm{~nm}), N_{1}=N_{2}=999$, while $N_{g}$, number of cell along $z^{\prime}$ in the gas region, consequently takes the values 4, 16, 64, 256, and 512. Each Maxwell spatial step $\Delta z_{M}$ is chosen such that $\Delta z_{M}<\lambda_{0} / 5$ where $\lambda_{0}=800 \mathrm{~nm}$ is the largest pulse wavelength considered in the computation.

As we solve one single TDSE per Maxwell cell of size $\Delta z_{M}$, a computation of $N_{g}$ TDSEs corresponds to a sample of gas of length $L=N_{g} \Delta z_{M}$, which contains $N_{g} \Delta z_{M} \mathcal{N}_{0}$ molecules, and the molecular density $\mathcal{N}\left(z^{\prime}\right)=\mathcal{N}_{0}$ is supposed to be constant. For numerical solving of TDSEs see Section 3.3. The molecular $\mathrm{H}_{2}^{+}$ TDSE spatial-temporal solver steps are denoted $\Delta x_{S}, \Delta y_{S}$ and $\Delta t_{S}$, which satisfy $\Delta t_{M} / \Delta t_{S} \gg 1, \Delta z_{M} / \Delta x_{S} \gg 1, \Delta z_{M} / \Delta y_{S} \gg 1$. In our computations $\Delta x_{S}=\Delta y_{S}=$ 0.3 a.u. with 200 (or 500) grid points in each direction. Such discretization establishes maximum free electron momentum $p_{x}=p_{y}=\pi / \Delta x_{S} \approx 10$ a.u. or equivalently a maximum energy $\mathcal{E}=p^{2} / 2$ a.u. $\approx 100$ a.u. $\approx 2700 \mathrm{eV}$. For $2 \mathrm{~d}$-TDSE the nuclear potential of $\mathrm{H}_{2}^{+}$is written as, see (2.3):

$$
\begin{gathered}
V_{C}\left(x, y, R_{0}\right)=-\left[\left(x-\frac{R_{0}}{2} \cos \theta\right)^{2}+\left(y-\frac{R_{0}}{2} \sin \theta\right)^{2}+\varepsilon\right]^{-1 / 2} \\
-\left[\left(x+\frac{R_{0}}{2} \cos \theta\right)^{2}+\left(y+\frac{R_{0}}{2} \sin \theta\right)^{2}+\varepsilon\right]^{-1 / 2}
\end{gathered}
$$

where $\theta \in\left[0^{\circ}, 90^{\circ}\right]$ defines the angle between the molecular axis of $\mathrm{H}_{2}^{+}$and the $x$-axis, with $R_{0}=2$ a.u. the internuclear distance. The value of $\varepsilon=4.5$ is used to reproduce the computational energy of the ground state of a $\mathrm{H}_{2}^{+}$-molecule $\approx-0.58$ a.u. The time step for solving the TDSEs is chosen as $\Delta t_{S}=\Delta t_{M} / 20 \approx 0.036$ a.u.

Parallel computing strategy described in Section 3.6.1 is used for solving the large set of 2-d TDSEs, see [77]. 


\subsubsection{Physical Results}

\section{Linearly Polarized Pulse}

We first study the propagation of LP-laser pulses in the $\mathrm{H}_{2}^{+}$-gas modeled by 2-d TDSEs. The initial laser pulse is chosen as follows: $E_{x}=0, B_{y}=0$, with $E_{y}$ and $B_{x}$ defined in (2.11), at the intensity $I_{y}=5 \times 10^{13} \mathrm{~W} / \mathrm{cm}^{2}=1.42 \times 10^{-3}$ a.u. The $\mathrm{H}_{2}^{+}$-molecules are oriented along the $y$-axis (i.e. parallel to $E_{y}$, Fig 2.5). In the following simulations the gas density is assumed to be constant in space and time. The computation grid for the TDSEs is a $200 \times 200$-point grid. In Fig. 4.3, we report the harmonic spectrum, up to the 11th harmonic, of the intensity of the electric field, $I(\omega)=I_{x}(\omega)+I_{y}(\omega) \propto\left|\hat{E}_{x}(\omega)\right|^{2}+\left|\hat{E}_{y}(\omega)\right|^{2}$, as a function of the propagation length of the LP-pulse.

Notice, in case of initial LP-pulse $I_{x}(\omega) \ll I_{y}(\omega)$, i.e. " $(\omega, I(\omega)) \approx\left(\omega, I_{y}(\omega)\right)$ ". In Fig. 4.4, we report the harmonic spectrum (in the same interval, i.e. up to the 11th harmonic) of the $y$-component of the dipole moment $d_{y},\left(\omega,\left|\hat{d}_{y}(\omega)\right|^{2}\right)$, of a molecule

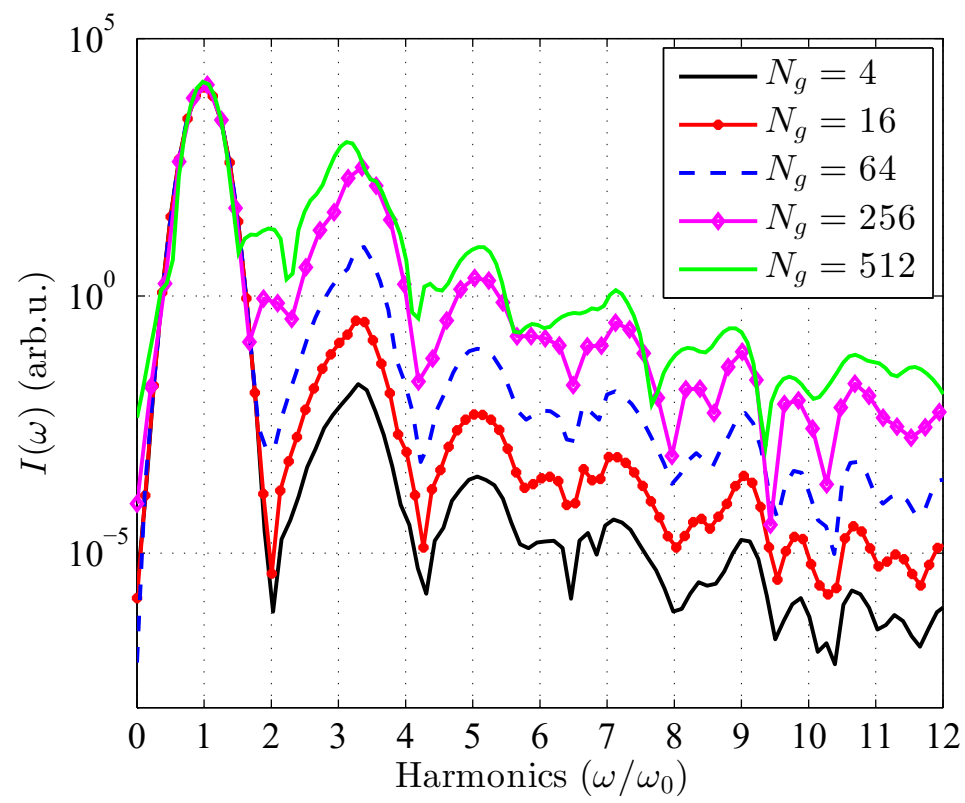

FiguRE 4.3: Intensity of the low order electric field harmonic spectrum for different "gas region lengths" $L=\Delta z_{M} N_{g}$ for LP-pulses $\left(\lambda=800 \mathrm{~nm}, I=5 \times 10^{13} \mathrm{~W} / \mathrm{cm}^{2}, \Delta z_{M}=100\right.$ a.u.) (c) 2016 American Physical Society 
located at the right-end of the gas region, for different "gas region lengths". Fig. 4.5 also illustrates the whole spectrum $(\omega, I(\omega))$ up to the cutoff region about the 30th harmonic. According to the Nyquist-Shannon sampling theorem the maximum number of harmonic we can consider is given by

$$
N_{\mathrm{Har}} \approx \frac{\pi}{\omega_{0} \Delta t_{M}}
$$

so that for $\omega_{0}=0.057$ a.u. and $\Delta t_{M}=\Delta z_{M} / c \simeq 0.73$ a.u. we get $N_{\mathrm{Har}}=75$.

In Fig. 4.6, we report the intensities, $I(\omega)$, of the first generated odd harmonics $(3,5,7,9)$ on a logscale, as a function of the propagation length. These results are consistent with (1.67) as well as with those of [80], where the quadratic scaling harmonic intensities/propagation length, was observed in a 1d-1d model.

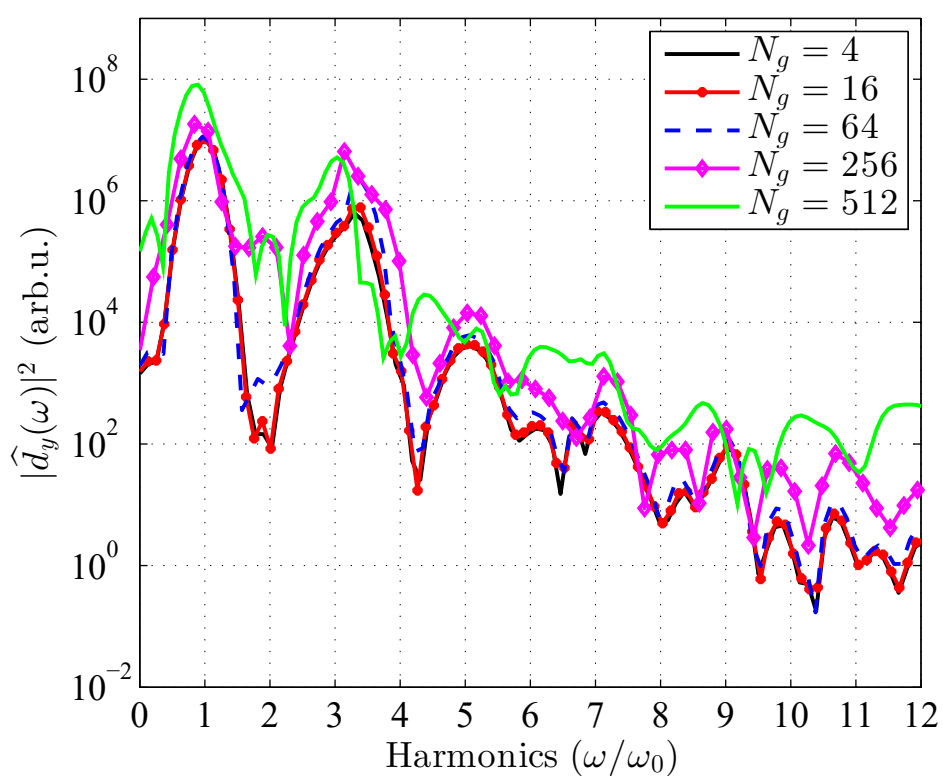

FIGURE 4.4: Intensity of harmonics of the dipole moment $\widehat{d}_{y}(\omega)$ of molecule in the right-end of the gas region for different propagation lengths $L=\Delta z_{M} N_{g}$ of LP-pulses ( $\lambda=800 \mathrm{~nm}$, $I=5 \times 10^{13} \mathrm{~W} / \mathrm{cm}^{2}, \Delta z_{M}=100$ a.u.) (c) 2016 American Physical Society 


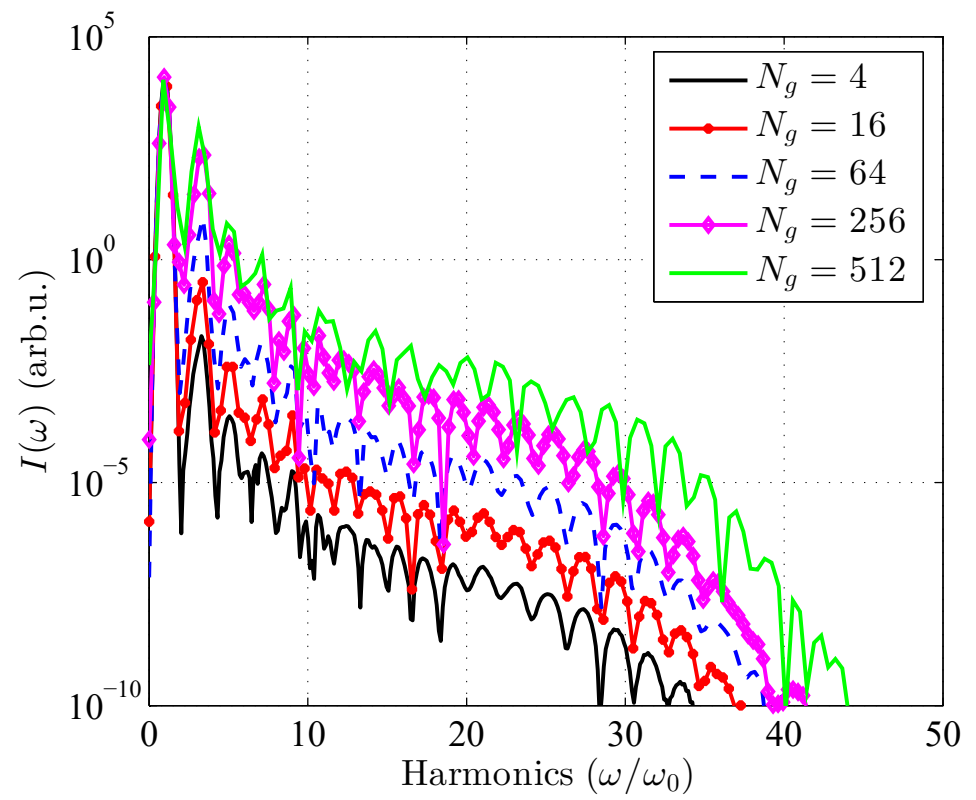

FIGURE 4.5: Intensity of harmonics of the transmitted electric field for different "gas region lengths" $L=\Delta z_{M} N_{g}$ for LP-pulses. (c) 2016 American Physical Society

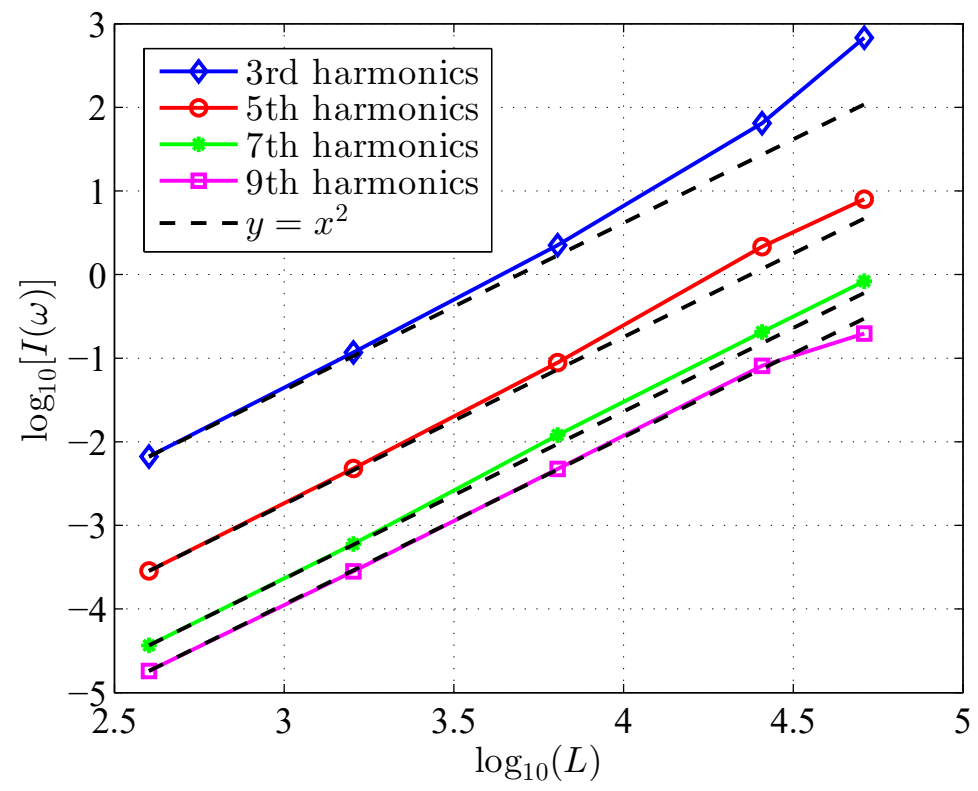

FiguRE 4.6: Intensity of the low order harmonics as a function of the propagation length $L=\Delta z_{M} N_{g}$ of the LP-pulse. (C) 2016 American Physical Society 


\section{Circularly Polarized Pulse}

The initial CP-pulse (2.11) has a total intensity $I=10^{14} \mathrm{~W} / \mathrm{cm}^{2}$, i.e. $5 \times 10^{13} \mathrm{~W} / \mathrm{cm}^{2}$ per each component of electric field, $E_{x}$ and $E_{y}$. The $\mathrm{H}_{2}^{+}$-nuclei lie along the $y$-axis. Since the CP-electron radius becomes $r(E)=3.7 E_{0} / \omega^{2} \approx 44$ a.u. [128], the TDSE grid is now $500 \times 500$-point grid or $150 \times 150 a_{0}^{2}$. In Table 4.1 , we report the propagation time, as well as the $L^{2}$-norm of the wavefunction "after the pulse" - both LP and CP cases for comparison. All the results in the table and on the following graphs refer to the case of homogeneous density, except when non-homogeneous density is explicitly specified.

\begin{tabular}{|c|c|c|c|}
\hline$N_{g}$ & Final time (a.u.) & $\|\psi\|_{L^{2}}^{2}(\mathrm{LP})$ & $\|\psi\|_{L^{2}}^{2}(\mathrm{CP})$ \\
\hline \hline 4 & 869.815 & 0.972799 & 0.952074 \\
\hline 16 & 878.485 & 0.973138 & 0.949644 \\
\hline 64 & 913.162 & 0.972781 & 0.94984 \\
\hline 256 & 1051.87 & 0.972088 & 0.947415 \\
\hline 512 & 1236.81 & 0.971847 & 0.945787 \\
\hline \hline
\end{tabular}

TABle 4.1: Propagation time and $L^{2}$-norm of the wavefunction after impact of the laser pulse. (c) 2016 American Physical Society

In Fig. 4.7, we report the electric-field harmonic spectrum intensity (first 11th harmonics), $I(\omega)$, as a function of the propagation length of the CP-pulse in the gas. In Fig. 4.9, we show the same spectrum up to 40th harmonic. In Fig. 4.8, we report the harmonic spectrum of the squared absolute value of the dipole moment (see (2.4)) $\left(\omega,|\hat{d}(\omega)|^{2}\right)$, of a molecule located in the right boundary of the gas region, for different "gas region lengths".

In Fig. 4.10, we report the temporal evolution of the squared absolute value of the wavefunction of a molecule in the left end of the gas region. In order to avoid spurious reflections at the computational domain boundary, we impose absorbing boundary conditions with artificial potential $U_{a b s}=-V_{1}(x, y)-i V_{2}(x, y)$, such that the positive definite functions $V_{1}$ and $V_{2}$ are decreasing linearly in $x$ or $y$ while 


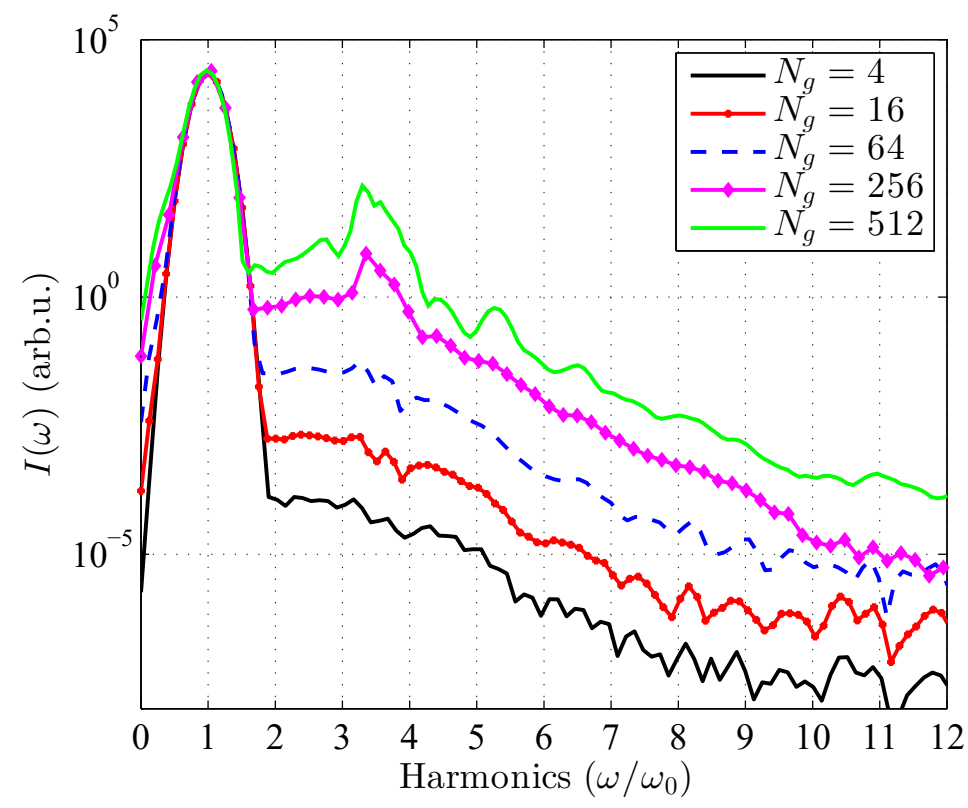

FiguRE 4.7: Low order harmonic spectrum of the transmitted electric field for different "gas region lengths" $L=\Delta z_{M} N_{g}$ for CP-pulse. (C) 2016 American Physical Society

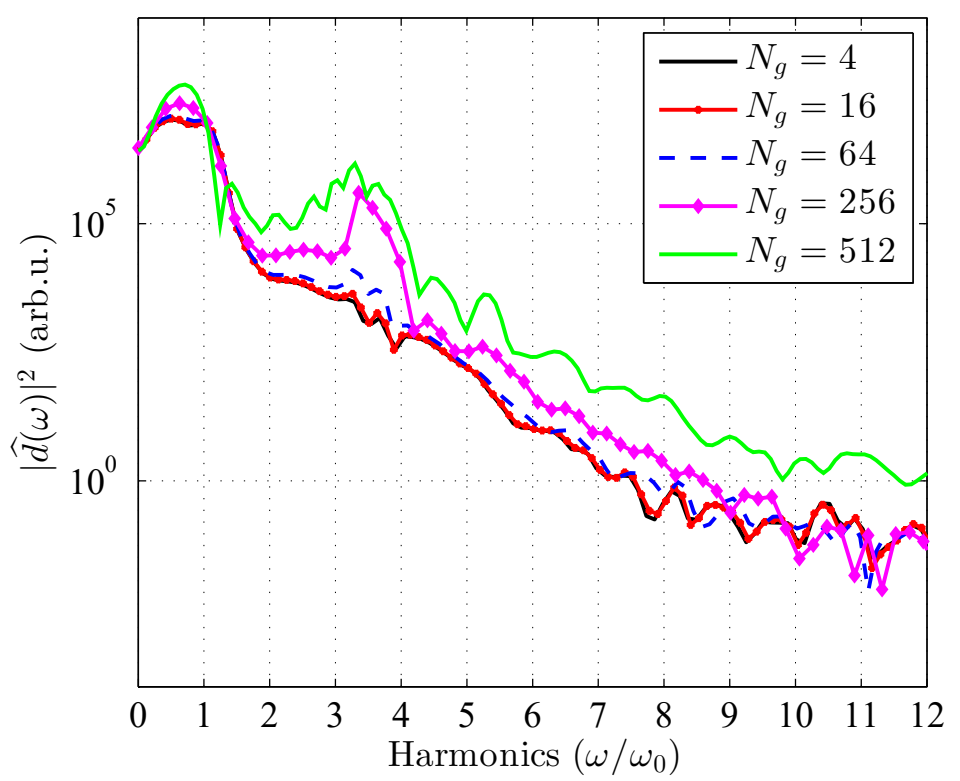

Figure 4.8: Low order harmonic of the dipole moment of aligned $\mathrm{H}_{2}^{+}$-molecules in the right (exit) end of the gas region for different propagation lengths $L=\Delta z_{M} N_{g}$ of CP-pulses. (C) 2016 American Physical Society 


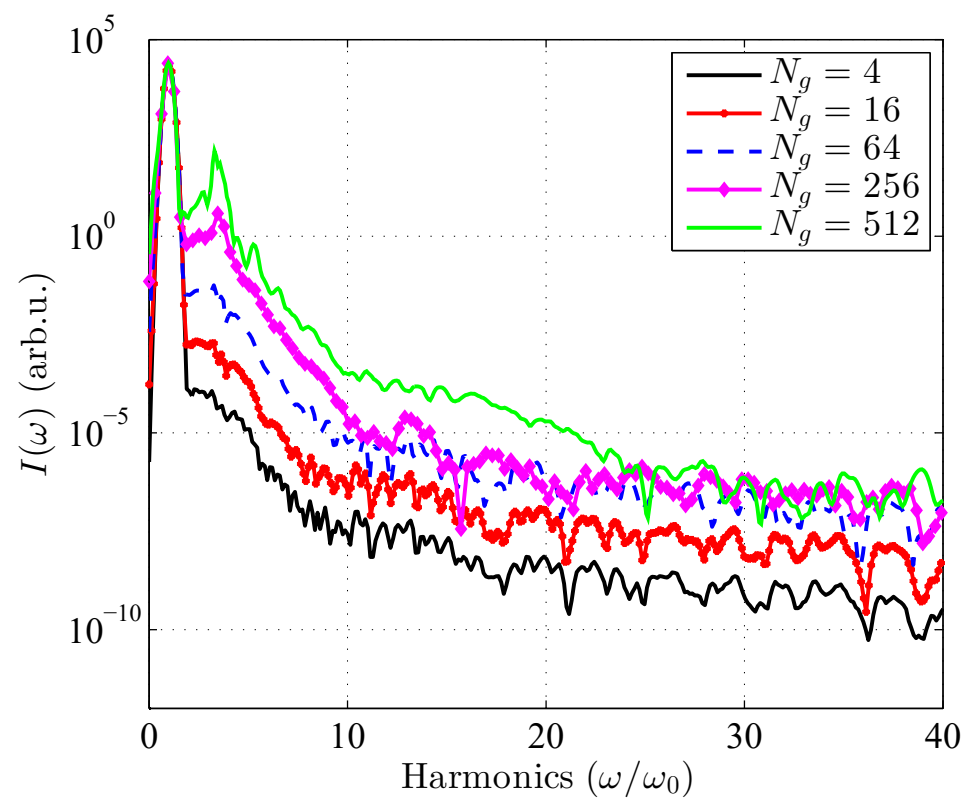

Figure 4.9: Total harmonic spectrum of the transmitted electric field for different "gas region lengths" $L=\Delta z_{M} N_{g}$ for CP-pulse. (c) 2016 American Physical Society

approaching the edges of the computational domain, defined by a $500 \times 500$-point grid, see Subsection 3.3.3.

In Fig. 4.11, we report the intensities, $I(\omega)$, of the first generated odd harmonics (3rd, 5th, 7th, 9th) on a logscale, as a function of the propagation length of the CPpulse in the gas. These results suggest that the quadratic scaling (intensity/propagation length) that was observed and justified in the LP-case, is also satisfied in the CP-case for the first odd harmonics.

The above results illustrate that, high frequency photons could coherently be added in the $\mathrm{CP}$-case, to generate coherent shorter pulses than the probe pulse. In Fig. 4.12 , we report the cosinus of the dipole phase difference of $\hat{d}_{x}\left[(2 k+1) \omega_{0}\right]$ and $\hat{d}_{y}\left[(2 k+1) \omega_{0}\right]$ at $k=1,2,3,4,5$ as a function of the propagation length, for a molecule located at the left-end (incident) region and a molecule located at the rightend (outgoing) of the gas region, that is $\cos \left\{\nu_{x}\left[(2 k+1) \omega_{0}\right]-\nu_{y}\left[(2 k+1) \omega_{0}\right]\right\}$, where $\nu_{x}\left[(2 k+1) \omega_{0}\right]:=\operatorname{Arg}\left[\widehat{d}_{x}\left(2(k+1) \omega_{0}\right)\right]$ and $\nu_{y}\left[(2 k+1) \omega_{0}\right]:=\operatorname{Arg}\left[\widehat{d}_{y}\left(2(k+1) \omega_{0}\right)\right]$. When this value is close to 0 , as in the case of 5 th and 9 th harmonics, we conclude 

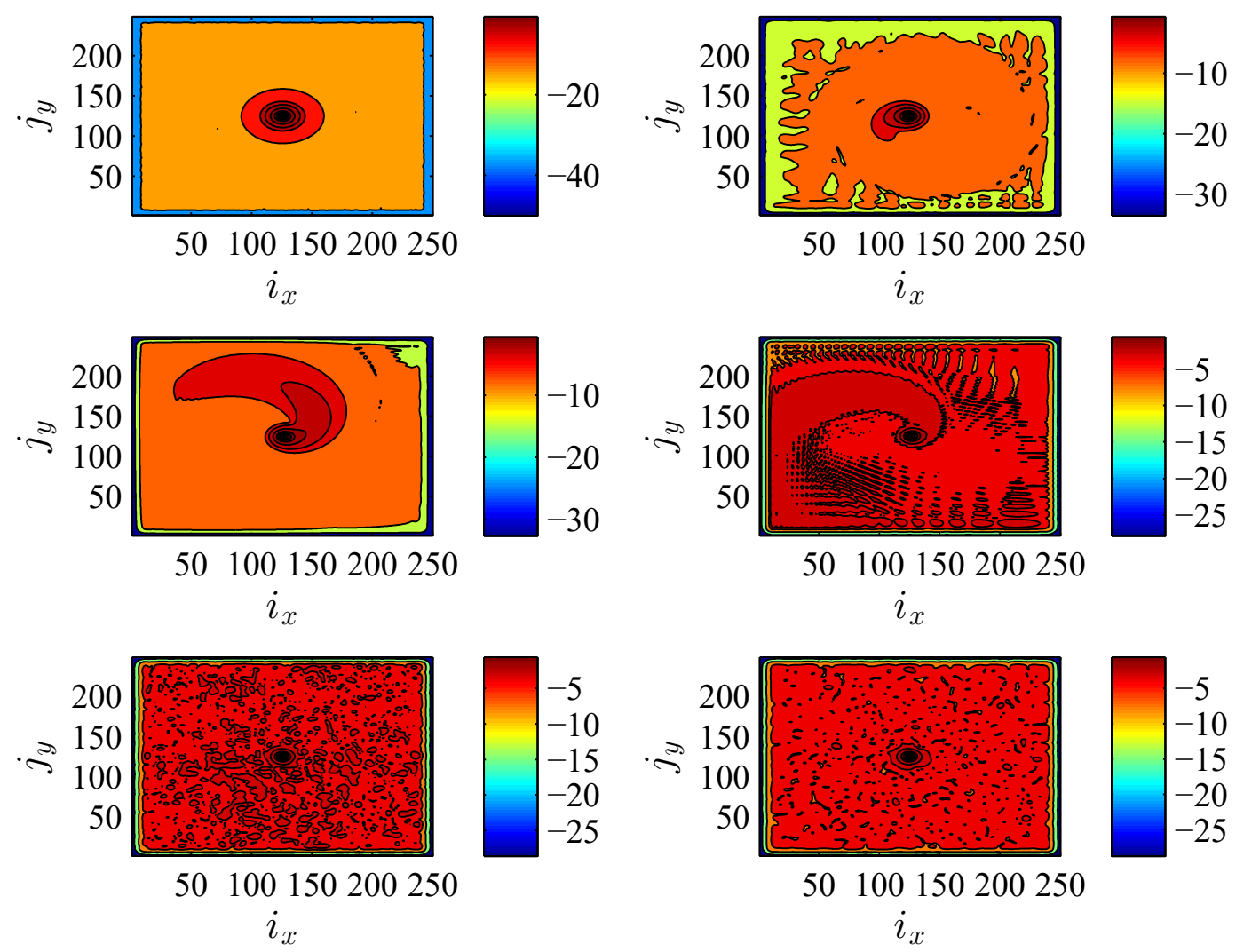

Figure 4.10: The level sets of electron density $\log \left(|\psi(x, y)|^{2}\right)$ for a $\mathrm{H}_{2}^{+}$-molecule in the left (entering) end of the gas region at six consecutive times: $t=0,302,349,451,757,1237$ a.u., time(a.u.) $=24$ asec. Total number of TDSEs, $N_{g}=512$. (C) 2016 American Physical Society

to the emission of circularly polarized photons of frequency $2(k+1) \omega_{0}$.

Numerical simulations of a MS model allows then for a nonperturbative calculation of the macroscopic polarization in MEs using the solution of $\mathrm{H}_{2}^{+}$-molecular TDSEs subject to an intense laser pulse. The MS-model permits the accurate observation of the HHG and nonlinearities through multiphoton-ionization, as well as the inclusion of corresponding coherent effects at the macroscopic scale. Although it is theoretically possible to rigorously derive the macroscopic nonlinear polarization from laser-molecule TDSEs in the case of linearly polarized pulses, the circularly polarized case is more complex. Through the numerical MS model, we have obtained the dipole harmonic intensities and their phase as a function of the pulse propagation length. We 


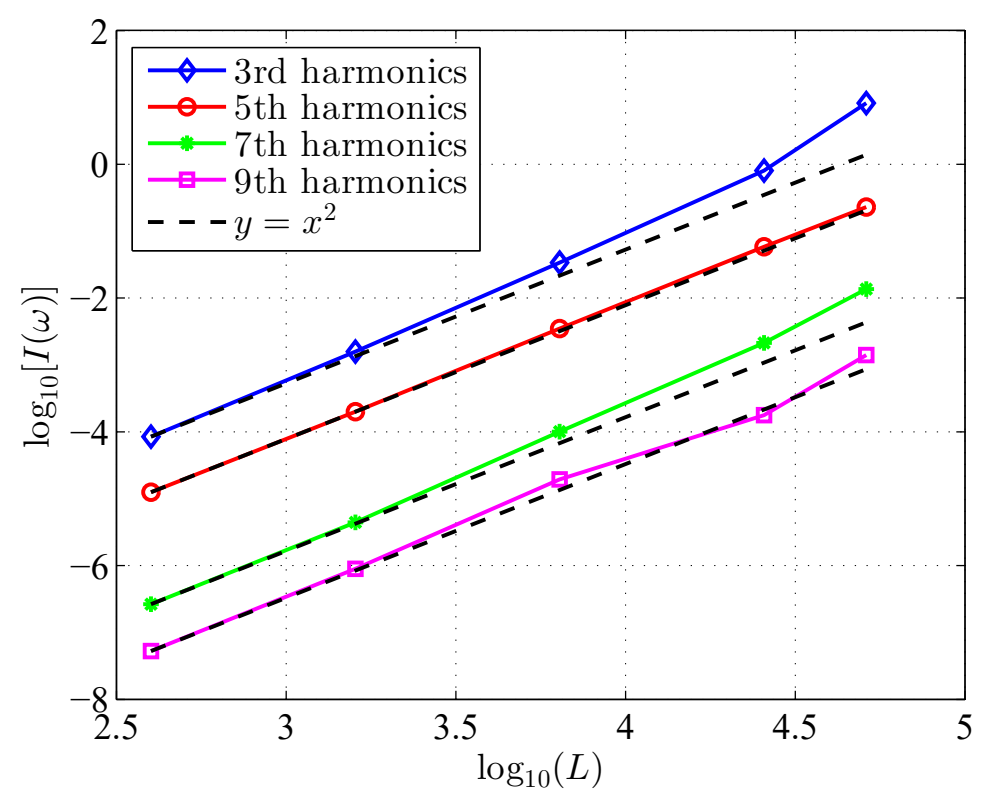

FiguRE 4.11: Intensity of the low order harmonics as a function of the propagation length $L=\Delta z_{M} N_{g}$ of the CP-pulse in gas. (c) 2016 American Physical Society

have shown that, low order harmonics (up to $N \approx 11$ th), it is possible to coherently generate circularly polarized pulses shorter than the incoming incident circular pulse. Note that the third harmonic that in atmospheric linear laser filamentation $[33,117]$ is an important nonlinear optical emission, is also observed in the LP-pulse propagations reported in Figs. 4.3, 4.4, 4.5. Of interest in our work, this now appears also in circular polarization, a phenomenon never observed before from our knowledge. In fact, Fig. 4.7 shows that the third harmonic in circular polarization becomes more dominant with increasing the gas density. Macroscopic propagation effects on higher order circular harmonics, and circularly polarized filaments, where strong inversion and laser emission has been observed $\mathrm{N}_{2}^{+}[41]$ are a future research direction. 

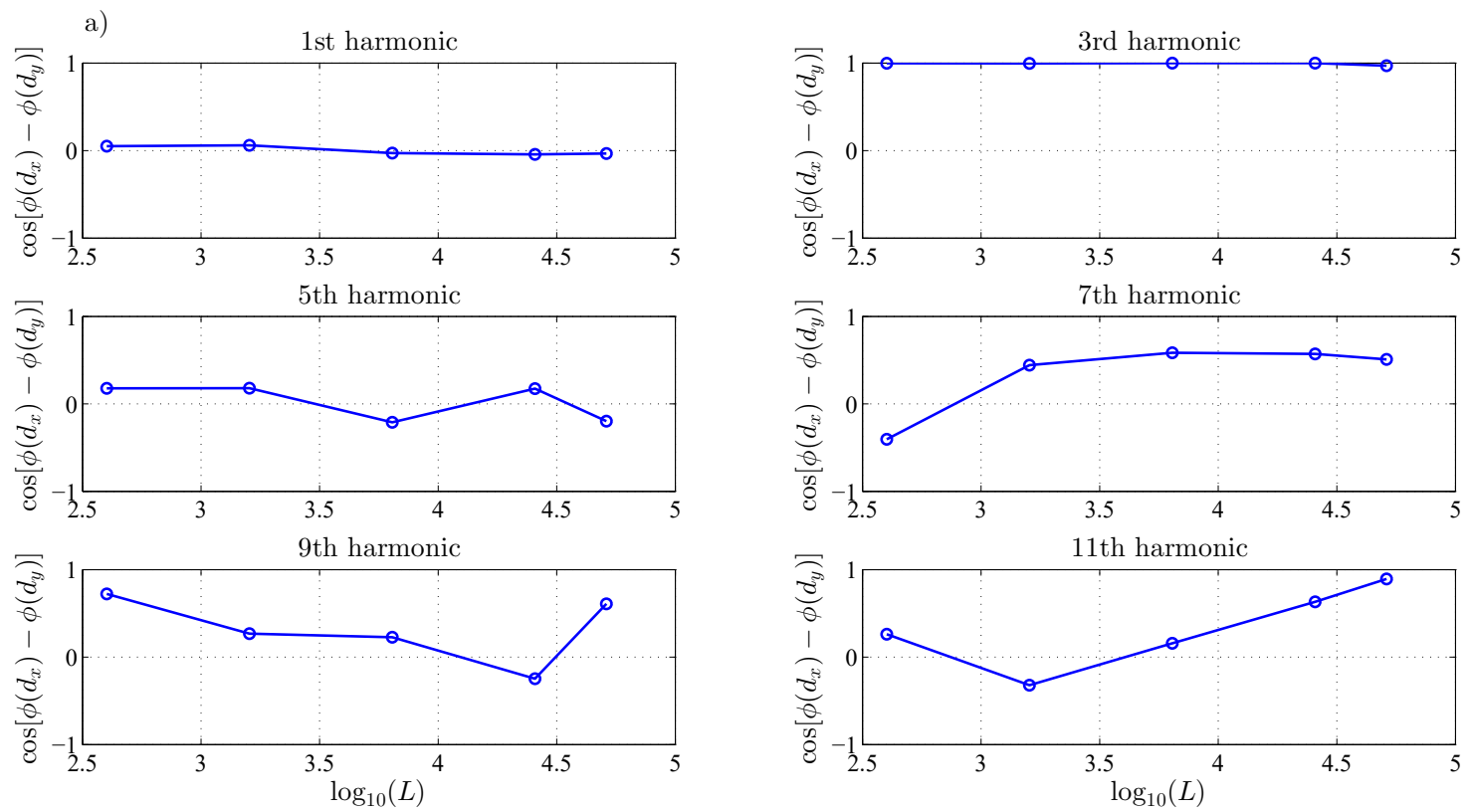

b)
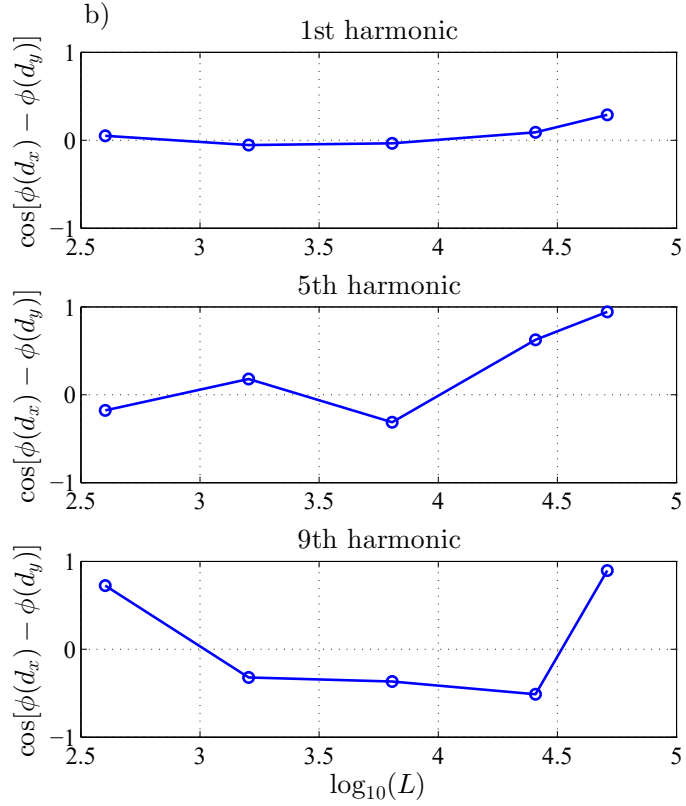
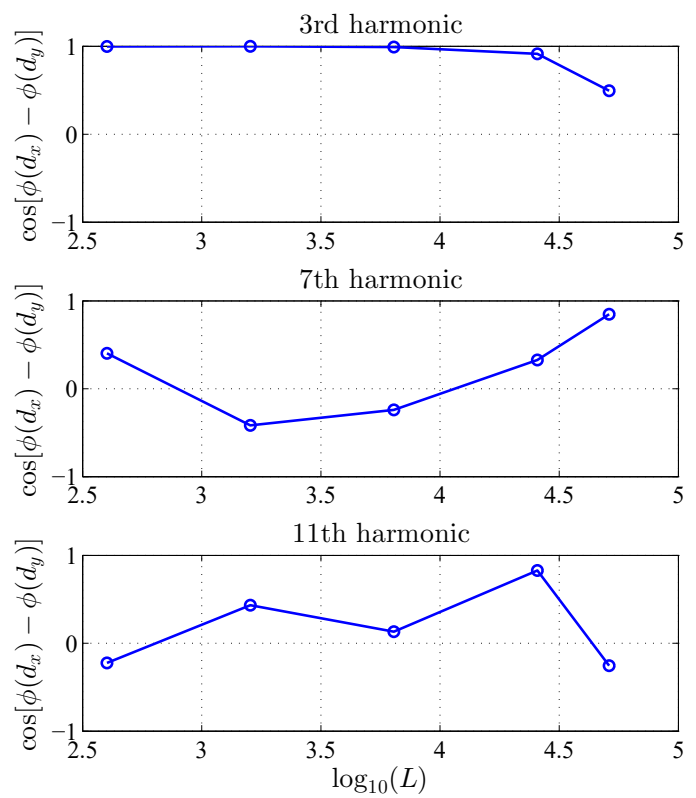

FiguRE 4.12: $\cos \left[\phi\left(\hat{d}_{x}\left((2 k+1) \omega_{0}\right)\right)-\phi\left(\hat{d}_{y}\left((2 k+1) \omega_{0}\right)\right)\right]$, for $k=0,1,2,3,4,5$ as a function of propagation length $L=\Delta z_{M} N_{g}$ of the CP-pulse, for $\mathrm{H}_{2}^{+}$-molecules at the leftend (incident) (a) and at the right-end (outgoing) (b) of the gas region. (c) 2016 American Physical Society 


\subsection{1d-2d MASP Model with the Polarization Evolution Equation}

In this section we present the results obtained within the MASP model supplemented by the polarization evolution equation. As we are not only concerned about the spectra of the generated harmonics but also about evolution of the pulse shapes in time and space, we use the Lax-Wendroff method, being more precise compared to the Lax-Friedrichs method used in Section 4.1.

\subsubsection{Testing the Models in Perturbative Regime}

We begin by presenting two numerical models:

Model 1 is based on including in the 1d Maxwell's equations the polarization represented as a perturbative expansion (3.36). The corresponding numerical scheme is provided in Section 3.1.3.

Model 2 consists of 1d Maxwell's equations (3.28) supplemented by the polarization evolution equation (2.36) (neglecting the free electrons currents). Note that by implementing this model, we do not solve the Schrödinger equation in the first cells of each subdomain, but use there the perturbative expansion for $\partial_{t} \mathbf{P}\left(z_{\alpha}^{\prime}, t\right)$. Then we apply the evolution equation for all remaining cells following the procedure described in Section 2.3.2. The numerical schemes for Model 2 are represented by (3.33) with polarization computed from (3.105) and $(3.106)$.

It is natural to expect that the both models will yield similar results, as in both cases we deal with the same phenomenon: propagation of the laser pulse through the molecular gas in the perturbative regime. Model 1 seems to be simpler and easier to implement, e.g. as sort of the HOKE-model, but it cannot be coupled with nonper- 
turbative MASP model. On the other hand, Model 2 does allow coupling with the MASP model; note that for this purpose we would need to replace the perturbative expression for polarization with nonperturbative computing of polarization through solving the TDSEs in the first cells of the subdomains. As we discussed in Section 2.3, the purpose of such coupling is to reduce the overall computational cost of the enriched model as compared to the pure MASP model. Hence our goal is to make sure that Model 2 works properly at least in the case of perturbative approximation for polarization. If Model 2 passes this test, it will make sense to combine it with the MASP model.

As Models 1 and 2 do not require the computationally expensive solving of the TDSEs, all the simulations in Section 4.2.1 were performed on a personal computer in MATLAB, i.e. without resorting to the supercomputer. Recall that in Section 3.1.3, corresponding to Model 1, we considered only the third-order nonlinearity; however, functions $a\left(E_{y^{\prime}}\right)$ and $b\left(E_{y^{\prime}}\right)$, related to the model, can be easily extended to higher order nonlinearities, just as it was done when passing from (2.34) to (2.37). Thus, in our computations we applied the set of the odd susceptibility coefficients from $\chi^{(1)}$ to $\chi^{(9)}$ to the both models. The test values of these coefficients coincide in order of magnitude with those presented in [59]. Figs. 4.13 report the transmitted electric pulses after propagation through the gas region, computed with Model 1, and Model 2 at initial laser pulse intensities (a) $I=1.5 \times 10^{14} \mathrm{~W} / \mathrm{cm}^{2}$, and (b) $I=2 \times 10^{14} \mathrm{~W} / \mathrm{cm}^{2}$. As we see, the results of the both models are consistent, at least at first glance, and demonstrate reasonable behaviour of the pulse (compare with Fig. B.1, Appendix B): self-steepening resulting in the "shark-fin" like shape, see panel (a), and for higher intensity, see panel (b), the optical shock wave formation on the trailing edge.

To reduce the reflections of the EM-wave on the boundary of the gas region, we used the following density dependence on $z^{\prime}$ within the gas region: 


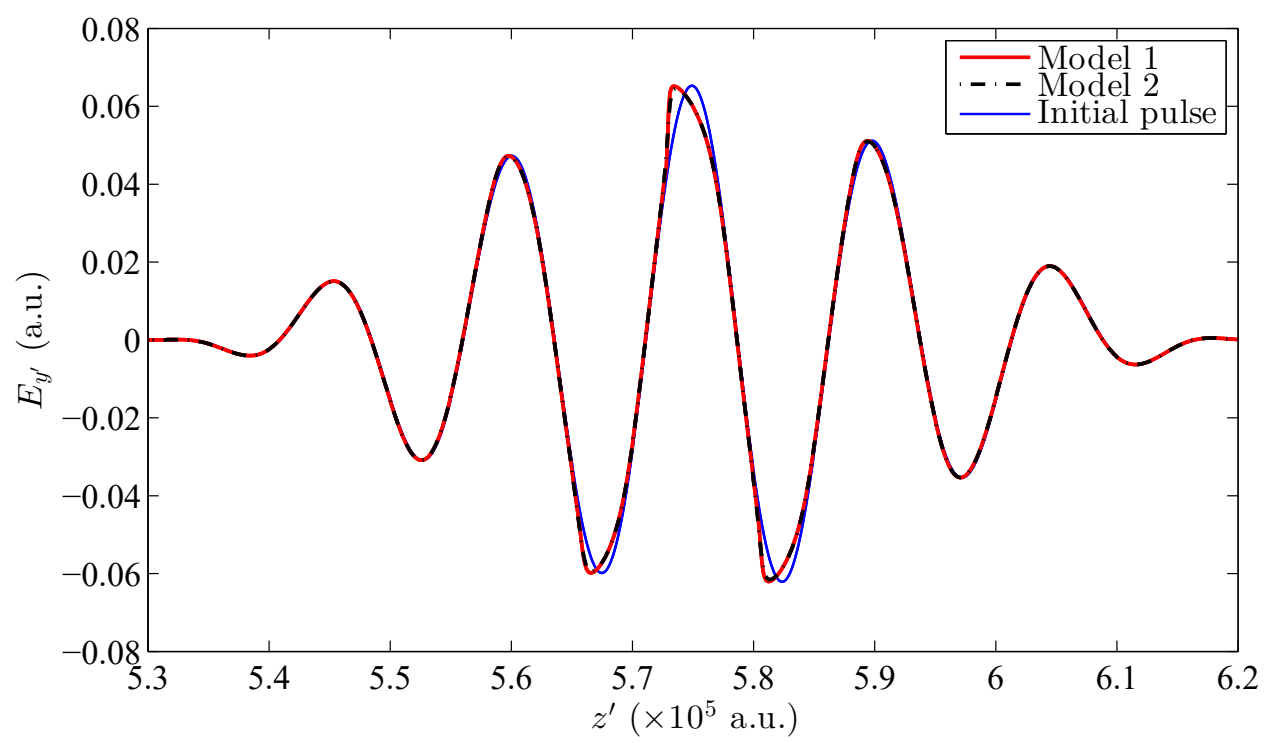

a)

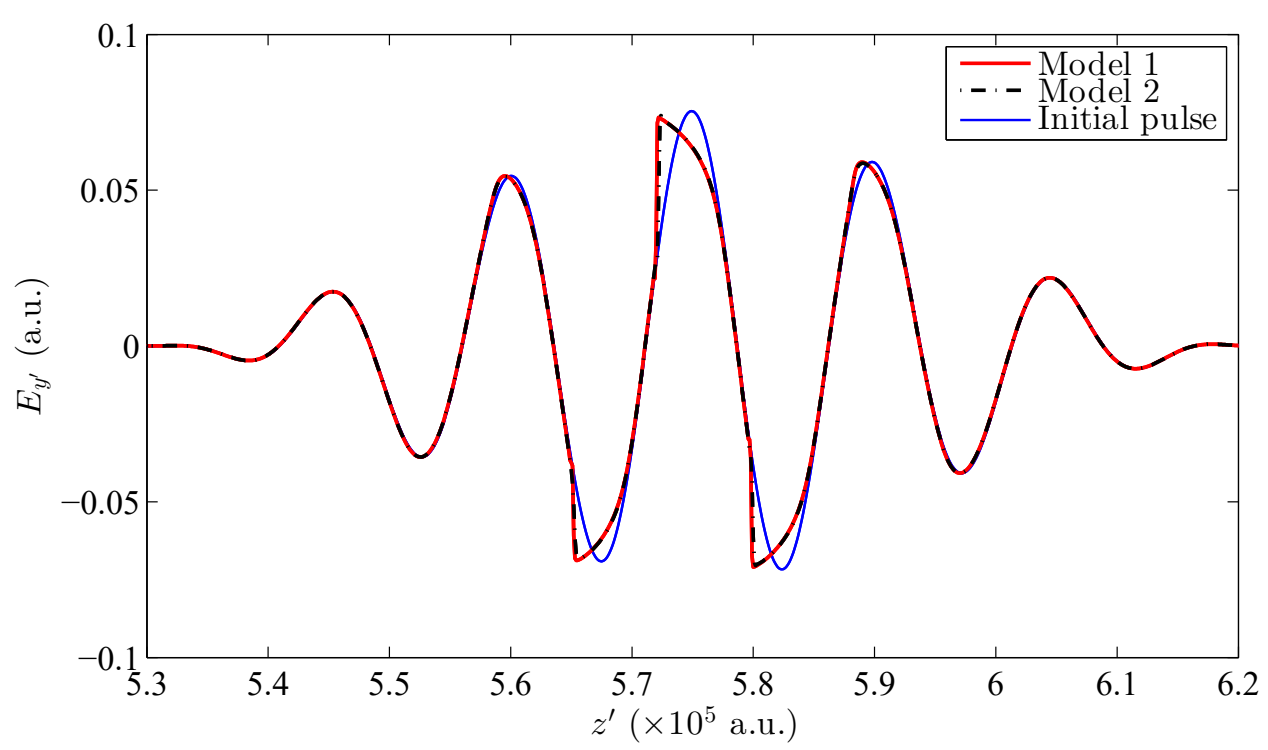

b)

Figure 4.13: Transmitted electric field as a function of space: time $T=3866$ a.u., the pulse is in the vacuum region $L_{2}$. Lengths of the vacuum regions: $L_{1}=10^{5}, L_{2}=1245 \times 10^{2}$ a.u., length of the gas region $L=4096 \times 10^{2}$ a.u. For Model 2: number of subdomains $N_{1}=64$, number of cells per each subdomain $N_{2}=64$. Space step $h=100$ a.u., time step $\tau=h / c=0.73$ a.u. Instantaneous susceptibilities: $\chi^{(1)}=1.8 \times 10^{-5}, \chi^{(3)}=2 \times 10^{-3}$, $\chi^{(5)}=1, \chi^{(7)}=300, \chi^{(9)}=10^{5}$. Initial pulse intensities: a) $I=1.5 \times 10^{14} \mathrm{~W} / \mathrm{cm}^{2}$ b) $I=2 \times 10^{14} \mathrm{~W} / \mathrm{cm}^{2}$. 


$$
\mathcal{N}\left(z^{\prime}\right)=\left\{\begin{array}{l}
\mathcal{N}_{0} \exp \left[\left(z^{\prime}-\left(L_{1}+L^{\prime}\right)\right)^{2} / \sigma^{2}\right], \quad z^{\prime} \in\left[L_{1}, L_{1}+L^{\prime}\right], \\
\mathcal{N}_{0} \exp \left[\left(z^{\prime}-\left(L_{1}+L-L^{\prime}\right)\right)^{2} / \sigma^{2}\right], \quad z^{\prime} \in\left[L_{1}+L-L^{\prime}, L_{1}+L\right] \\
\mathcal{N}_{0}, \quad z^{\prime} \in\left[L_{1}+L^{\prime}, L_{1}+L-L^{\prime}\right]
\end{array}\right.
$$

where $L^{\prime}$ is the width of the range near the boundaries, where the density increases (decreases) from 0 to $\mathcal{N}_{0}$ (or vice versa); $\sigma$ is another parameter characterizes the steepness of such transition. We use (4.5) in all computations of Section 4.2, because it gives smoother junction between 0 and $\mathcal{N}_{0}$ than linear dependence in (4.2).

In order to evaluate the difference between the solutions represented in Figs. 4.13 more precisely, in Fig. 4.14 we report the corresponding discrete $\ell_{2}$ - and $\ell_{\infty}$-norms at 10 equidistant time points $t_{n}(n=1 \ldots 10)$ :

$$
\begin{aligned}
\left\|E_{M_{1}}\left(\cdot, t_{n}\right)-E_{M_{2}}\left(\cdot, t_{n}\right)\right\|_{\ell_{2}} & =\left(h \sum_{i}\left|\left(E_{M_{1}}\right)_{i}^{n}-\left(E_{M_{2}}\right)_{i}^{n}\right|^{2}\right)^{\frac{1}{2}}, \\
\left\|E_{M_{1}}\left(\cdot, t_{n}\right)-E_{M_{2}}\left(\cdot, t_{n}\right)\right\|_{\ell_{\infty}} & =\max _{i}\left|\left(E_{M_{1}}\right)_{i}^{n}-\left(E_{M_{2}}\right)_{i}^{n}\right|
\end{aligned}
$$

where indexes $M_{1}$ and $M_{2}$ refer to Model 1 and Model 2 respectively. As we see, the higher intensity of the initial pulse (i.e. the stronger nonlinearity), the more noticeable difference between the solutions. From these graphs we can assume that at least one model is more or less unstable. Next Fig. 4.15 reports $\ell_{2}$-norms of the all solutions given in Fig. 4.13. We see that at space step $h=100$ a.u., Model 1 demonstrates modest local instability of the solutions in time due to the presence of the variable coefficients and nonlinearity in the scheme (3.41). It is noteworthy that more complicated Model 2 is stable, as $\left\|E_{M 2}\left(\cdot, t_{n}\right)\right\|_{\ell_{2}} /\left\|E_{M 20}\right\|_{\ell_{2}}<1$ for all $t_{n}$.

To estimate the order of convergence $p$ of the schemes for the both models, we refer to the definition of the error of a FDS at time moment $t_{n}=n \tau$ via discrete $\ell_{2}$-norm, see [113]:

$$
\left\|e\left(t^{n}\right)\right\|_{h}=\left(h \sum_{i}\left|E_{h, i}^{n}-E^{e x}\left(x_{i}, t_{n}\right)\right|^{2}\right)^{1 / 2}=C h^{p},
$$




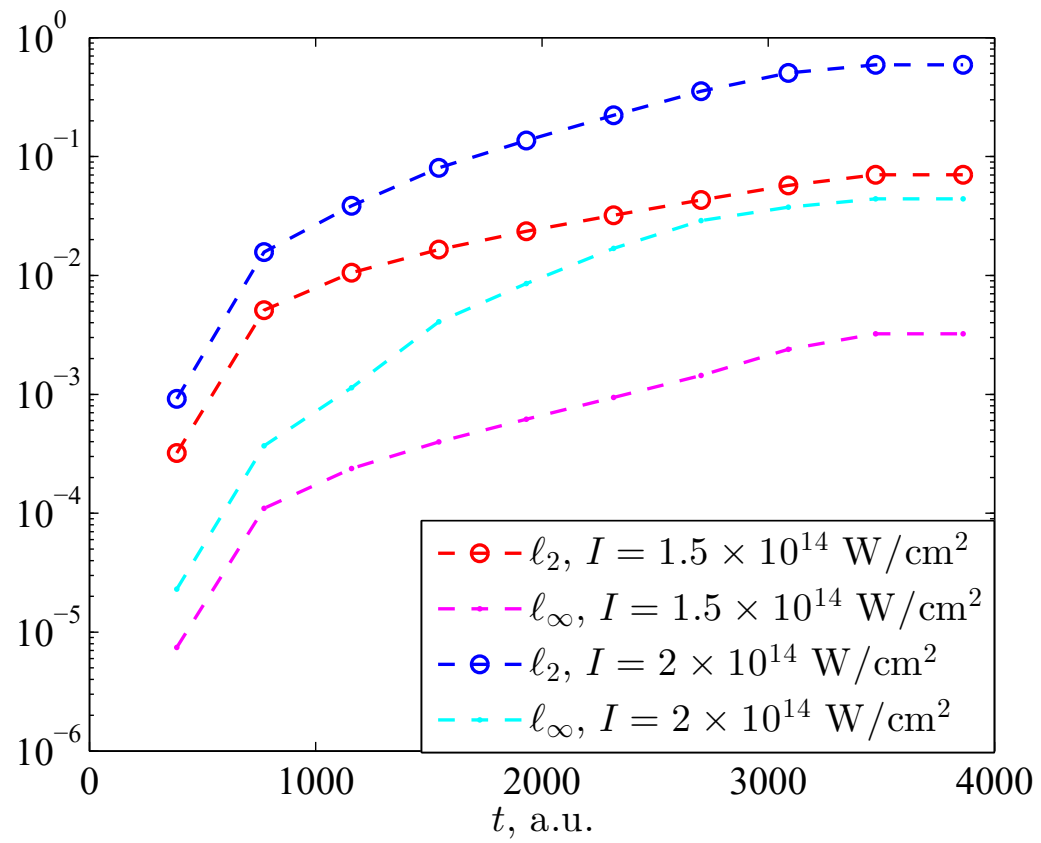

Figure 4.14: Discrete $\ell_{2}$ - and $\ell_{\infty}$-norms $(4.6),(4.7)$ in time of pointwise difference of the solutions represented in Figs. 4.13, space step $h=100$ a.u.

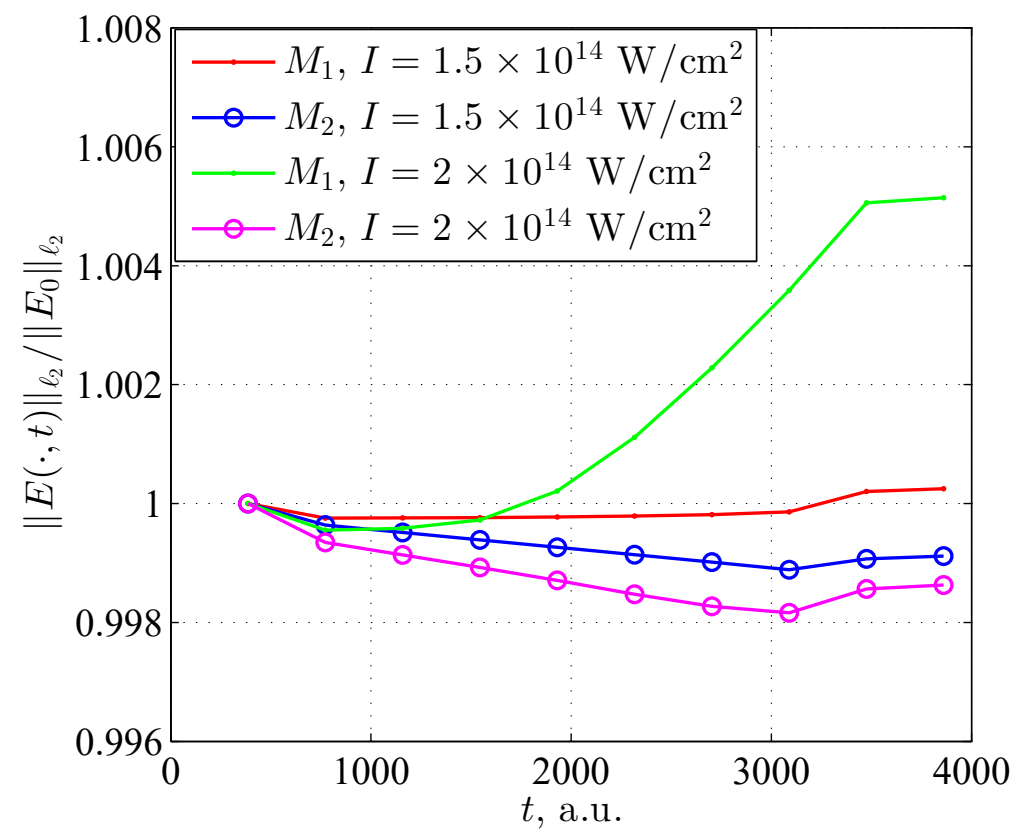

FiguRE 4.15: Discrete $\ell_{2}$-norms in time normalized on initial norms for solutions represented in Figs. 4.13. The initial norms: for $I=1.5 \times 10^{14} \mathrm{~W} / \mathrm{cm}^{2}:\left\|E_{0}\right\|_{\ell_{2}}=8.52$, and for $I=1.5 \times 10^{14} \mathrm{~W} / \mathrm{cm}^{2}:\left\|E_{0}\right\|_{\ell_{2}}=9.84$. 
where $E^{e x}(x, t)$ denotes the exact solution, and the sum is computed over all grid nodes with step $h$. Since the exact solution is unknown, we will try to estimate $p$ by computing the set of numerical solutions with reduced space steps: $h_{\nu}=h_{0} / 2^{\nu}$ $(\nu=0 \ldots 4)$. We introduce the ratio of two $\ell_{2}$-norms and use the triangle inequality:

$$
\begin{aligned}
R\left(t_{n}\right) & =\frac{\left\|E_{h}\left(\cdot, t_{n}\right)-E_{\frac{h}{4}}\left(\cdot, t_{n}\right)\right\|_{h}}{\left\|E_{\frac{h}{2}}\left(\cdot, t_{n}\right)-E_{\frac{h}{4}}\left(\cdot, t_{n}\right)\right\|_{\frac{h}{2}}} \geq \frac{\left\|\epsilon\left(t_{n}\right)\right\|_{h}-\left\|\epsilon\left(t_{n}\right)\right\|_{\frac{h}{4}}}{\left\|\epsilon\left(t_{n}\right)\right\|_{\frac{h}{2}}+\left\|\epsilon\left(t_{n}\right)\right\|_{\frac{h}{4}}}= \\
& =\frac{C h^{p}-C(h / 4)^{p}}{C(h / 2)^{p}+C(h / 4)^{p}}=2^{p}-1,
\end{aligned}
$$

where $E_{h}\left(\cdot, t_{n}\right)$ is a solution computed on the grid with step $h$. From here at $t_{n}$ we obtain the upper estimate on $p$ as

$$
p=\log _{2}\left[R\left(t_{n}\right)+1\right] .
$$

The following Tab. 4.2 represents thus evaluated order of convergence for Models 1 and Model 2, and for comparison in the case of free wave propagating in vacuum computed according to Lax-Wendroff scheme (3.33) with $\partial_{t} P_{y^{\prime}}=0$. We see that in all cases $p$ is very close to 2 , the consistency order of the Lax-Wendroff scheme.

The next Figs. 4.16 a), b) illustrate modifications of the pulse computed within Model 2. The red curve on the panel (a) represents the solution of the initial-boundary value problem for the polarization equation (2.36), while the blue curve shows the electric field of the laser pulse propagating through the gas region divided into 64 subdomains each containing 64 cells. We can observe the slight modification of the

\begin{tabular}{|c|c|c|c|}
\hline$h$, a.u. & Model 1 & Model 2 & Prop. in vac. \\
\hline \hline 100 & 1.9934 & 1.9945 & 1.9880 \\
\hline 50 & 1.9849 & 1.9944 & 1.9970 \\
\hline 25 & 1.9845 & 1.9932 & 1.9992 \\
\hline \hline
\end{tabular}

TABLE 4.2: Order of convergence evaluated according to (4.8) 


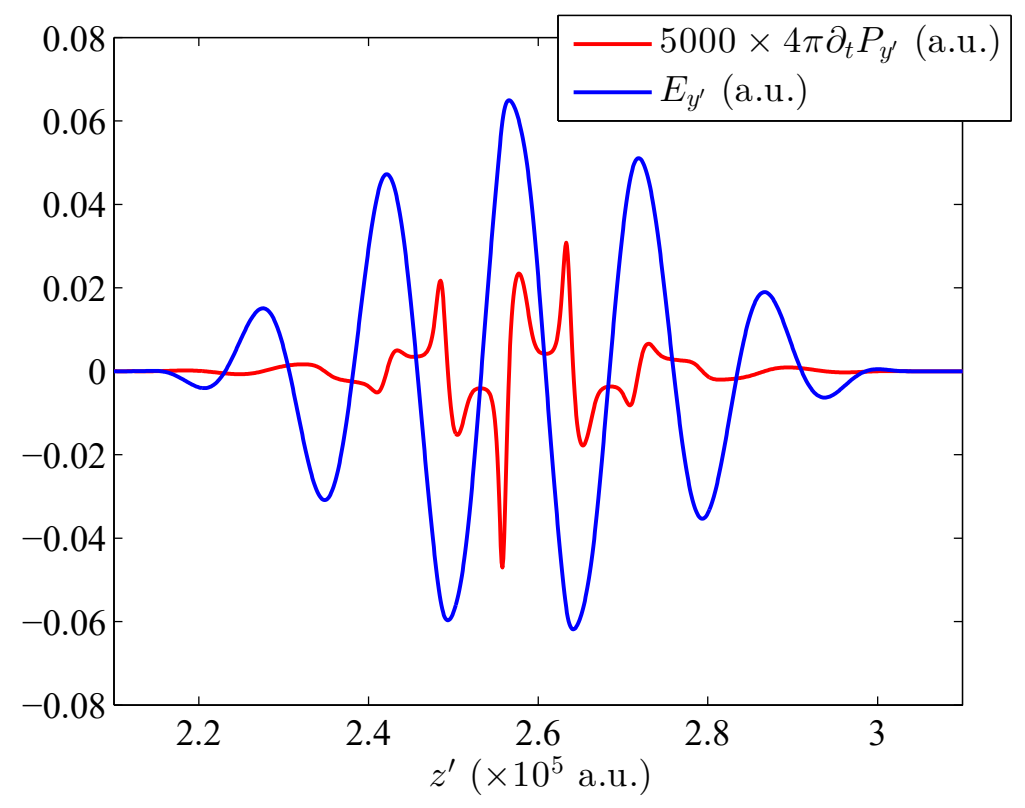

a)

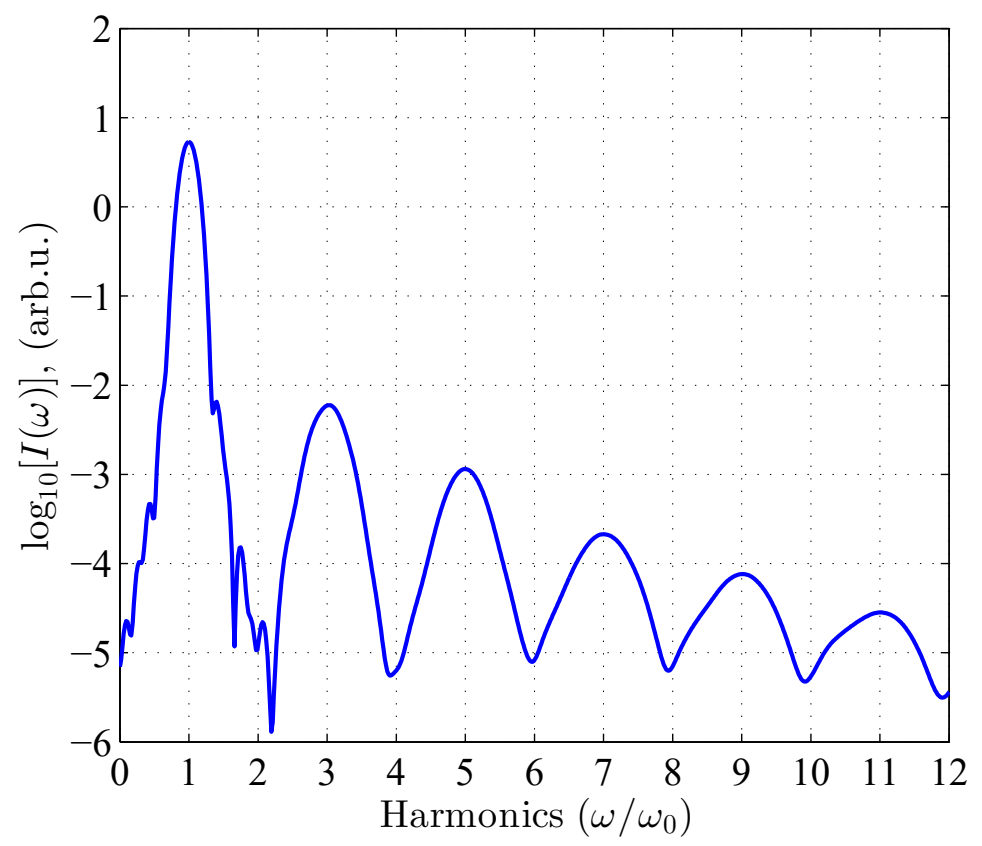

b)

Figure 4.16: Model 2: a) Electric field as a function of space at time moment $t=1530$ a.u., which corresponds to propagation through the gas region $L=4096$ a.u.; b) Spectral intensity of the laser pulse after propagation through the gas region, $t=3824$ a.u. Number of subdomains in the gas region $N_{1}=64$, number of cells per each subdomain $N_{2}=64$. Space step $h=100$ a.u., instantaneous susceptibilities: $\chi^{(1)}=1.8 \times 10^{-5}, \chi^{(3)}=2 \times 10^{-3}$, $\chi^{(5)}=1, \chi^{(7)}=300, \chi^{(9)}=10^{5}$. Initial pulse intensity $I=1.5 \times 10^{14} \mathrm{~W} / \mathrm{cm}^{2}$. 
pulse near its higher ridges. The output profile of the pulse after propagating through all the gas region corresponds to Fig. 4.13 a). The lower panel of Fig. 4.16 b) reports a spectrum of the transmitted pulse. We observe the set of odd harmonics that emerge after propagation of the pulse through the gas region, while initially the envelope contains the harmonic $\omega_{0}$ only.

The last figure of this section illustrates the dependence of the intensity of the $3^{\text {rd }}$ harmonic with respect to the propagation distance, evaluated within Models 1 and 2. As seen from Fig. 4.17, both models properly describe the "quadratic law", a well-known analytical result of the perturbative theory [24], see (1.67), which was also numerically extended on the case of nonperturbative regimes for not "too" long propagation distances, see Figs. 4.6 and 4.11, and [80,85].

We demonstrated in this Section that Model 2 is stable and convergent with order close to 2; the model can also adequately describe the features of pulse propagation in the gas. With this conclusion, we proceed to the results of the MASP model enriched by the evolution equations for polarization and free electrons density.

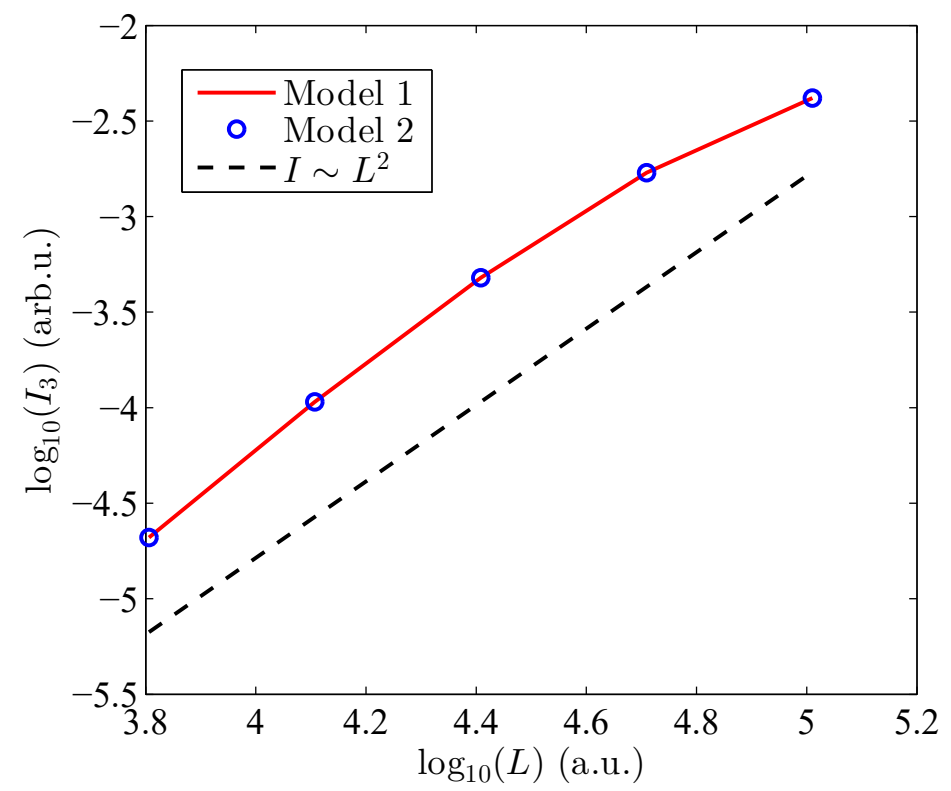

FigURE 4.17: Intensity of the third harmonic as a function of the propagation length in a gas: $L=\{64,128,256,512,1024\} \times 100$ a.u. computed within the Models 1 and 2 . 


\subsubsection{MS and MASP with 1d Polarization Equation}

We start by reporting the results of the MS model supplemented by the simple evolution equation described in Section 2.3.1, setting in addition $v_{g}=c$. The propagation path of the pulse in the gas region $L_{g}$, is divided into $N_{1}$ subdomains, each containing $N_{2}$ Maxwell's cells, see Fig. 2.7. At each Maxwell's time step, the TDSEs are solved several times (as $\Delta t_{M} / \Delta t_{S} \geq 20$ ) to provide the values of $\mathbf{P}$ in the first cells of each subdomain. The polarization in the remaining cells of the subdomains is supposed to be computed from the macroscopic transport equation (2.17) at the previous time step. The corresponding parallel computing strategy is presented in Section 3.6.2, see Fig. 3.10.

We report in Fig. 4.18 a), the spectral intensity of the LP-pulse, whose field vector E is parallel to the axis of $\mathrm{H}_{2}^{+}$-molecule. As we see, when $L^{2}$-norms of the wavefunctions are close to 1 (see the data in Tab. 4.1 corresponding to initial intensity $\left.I=5 \times 10^{13} \mathrm{~W} / \mathrm{cm}^{2}\right)$, the MS model enriched even by the simplest evolution equation provides decent approximations of spectra depending on the chosen partitions of the total number of cells in the gas region $N$ into number of subdomains $N_{1}$ and number of cells per each subdomain $N_{2}$ (see the legend). To make the comparison between the results of the enriched and the pure MASP models more convenient, we present in Figs. $4.18 \mathrm{~b})$, c) the dependencies $\Delta I(\omega)=\left[I(\omega)-I_{M A S P(\omega)}\right] / I_{M A S P}(\omega)$ smoothed by

applying the MATLAB function polyfit with $2^{\text {nd }}$-degree polynomial. Even such rough data processing allows making some key conclusions. From panel c) it is clear that the approximation between the models is better in the case of the smaller number of cells $N_{2}$ in the subdomain. However, from panel b) we can expect that there must be also a lower limit of number of cells in the subdomain for the application of the transport equation to be effective: the error in case of $N_{2}=8$ cells in the subdomain, the green curve on b), increases slower than in the case of $N_{2}=4$, red and blue curves. Evidently, this lower limit appears as a condition of smooth matching between the 

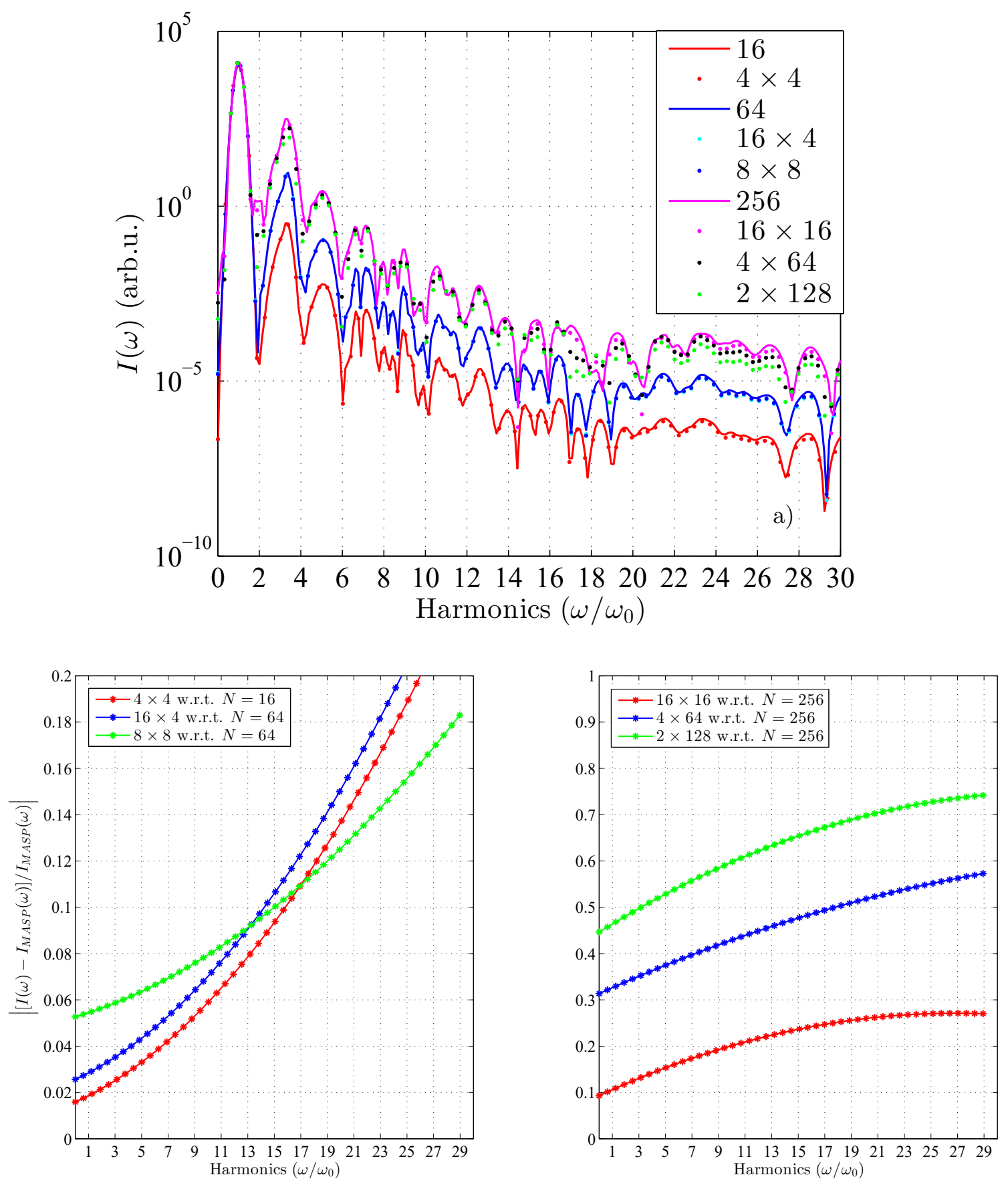

Figure 4.18: a) Spectral intensities of the electric field harmonics for different gas region lengths. Case of LP pulse: $\lambda=800 \mathrm{~nm}, I=5 \times 10^{13} \mathrm{~W} / \mathrm{cm}^{2}, \Delta z_{M}=100$ a.u., so that $L=100 \times N$ a.u. (see the legend for values of $N=N_{1} \times N_{2}$ ). The grid for solving 2-d TDSE is $300 \times 300$ with step $\Delta x_{S}=\Delta y_{S}=0.3$ a.u., gas density $\mathcal{N}=5.17 \times 10^{-5}$ a.u.; b), c) smoothed differences between the results of the enriched and pure MASP model. 
subdomains; if the length of each subdomain is too short, the "wave of polarization" cannot spread though the entire region without obstacles.

Within the same approach we also modeled the propagation of the CP initial pulse. In particular, we report in Fig. 4.19 the intensities of the first generated odd harmonics as functions of the propagation length in the gas. Comparing this graph with Fig. 4.11, we observe the concordance of the results within 5\%, even though the new data were obtained at a much lower cost of computing effort. Indeed, the suggested approach helps to reduce the computational complexity with respect to the original model. The table 4.3 shows that the processing times for the cases of the "pure" Maxwell-Schrödinger model and the MS model enriched by the polarization evolution equation are comparable, while in the first case the number of the engaged processors is up to 32 -fold. For example, in the case of CP initial pulse, the same spectrum was obtained for the same times but using 256 processors ("pure" model) and 16 processors (MS model coupling with the polarization evolution equation). Note that the computational times for the CP-case are higher, as we here used $500 \times 500$

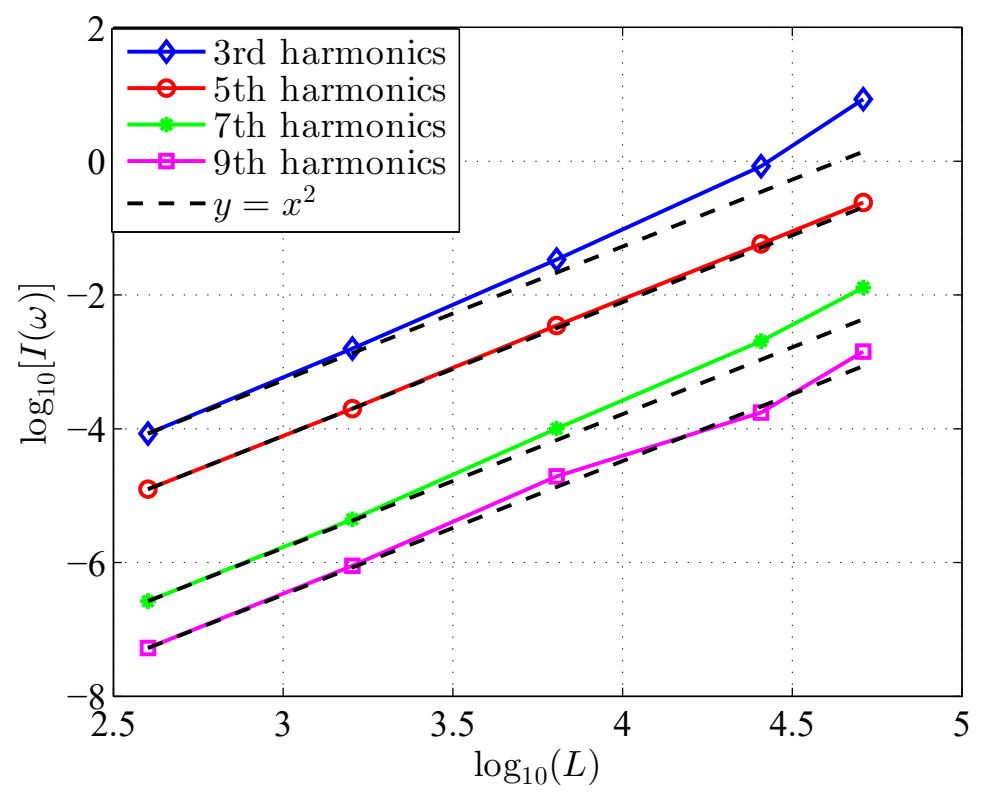

FiguRE 4.19: Intensity of the low order harmonics as a function of the propagation length $L=\Delta z^{\prime} \times N(N=4,16,64,16 \times 16$ and $32 \times 16)$ of the CP-pulse in gas. 


\begin{tabular}{|c|c|c|c|c|c|c|}
\hline$N_{1} \times N_{2}$ & 64 & $8 \times 8$ & 256 & $16 \times 16$ & $32 \times 16$ & $32 \times 32$ \\
\hline \hline Proc. time for LP pulse (h:m) & $03: 49$ & $03: 56$ & $04: 35$ & $04: 44$ & - & - \\
\hline Proc. time or CP pulse (h:m) & - & - & $13: 35$ & $13: 04$ & $15: 31$ & $21: 27$ \\
\hline \hline
\end{tabular}

TABLE 4.3: Model and computational parameters: number of subdomains $\left(N_{1}\right)$, number of the cells per subdomain $\left(N_{2}\right)$ and processing times.

points grids to solve the TDSE, while in LP-case $300 \times 300$ points grids are sufficient to properly computes of the wavefunctions.

In order to simulate the propagation of pulses with higher initial intensities, we resort to the more complicated polarization evolution equation that was described at Section 2.3.2 as (2.36), and then was tested in the previous Section 4.2.1 within Model 2. Further, we will compare the transmitted electric fields and spectra computed using the models enriched by the polarization equation with the the results of the "pure" MS/MASP models, considering the latter as reference data, see Figs. $4.20 \mathrm{a}$ ), b). Let us briefly discuss these figures. Note that in our computations we used different intensities of the initial pulse, $I_{1}=10^{14} \mathrm{~W} / \mathrm{cm}^{2}, I_{2}=5 \times 10^{14}$ $\mathrm{W} / \mathrm{cm}^{2}$ and different number densities $\mathcal{N}_{01}=1.63 \times 10^{-5}$ a.u. and $\mathcal{N}_{02}=5.17 \times 10^{-5}$ a.u. It is clear that the higher intensity of the pulse and density of the gas, the greater influence of the free electron currents on dynamics of the process. For example, in case of initial pulse with $I_{1}, L^{2}$-norm of the wavefunction is $\|\psi\|_{L^{2}}^{2} \approx 0.66$, while in case of $I_{2}$ we obtained $\|\psi\|_{L^{2}}^{2} \approx 10^{-6}$, which results in significant difference in the free electron number density (2.30). That is why the computations involving $I_{2}$ were performed with the MASP model. Recall that unlike the MS model, the MASP model takes into account the currents of free electrons. Engaging of the MASP approach in case of $I_{1}$ is not so crucial, but we still used this more generic model. 


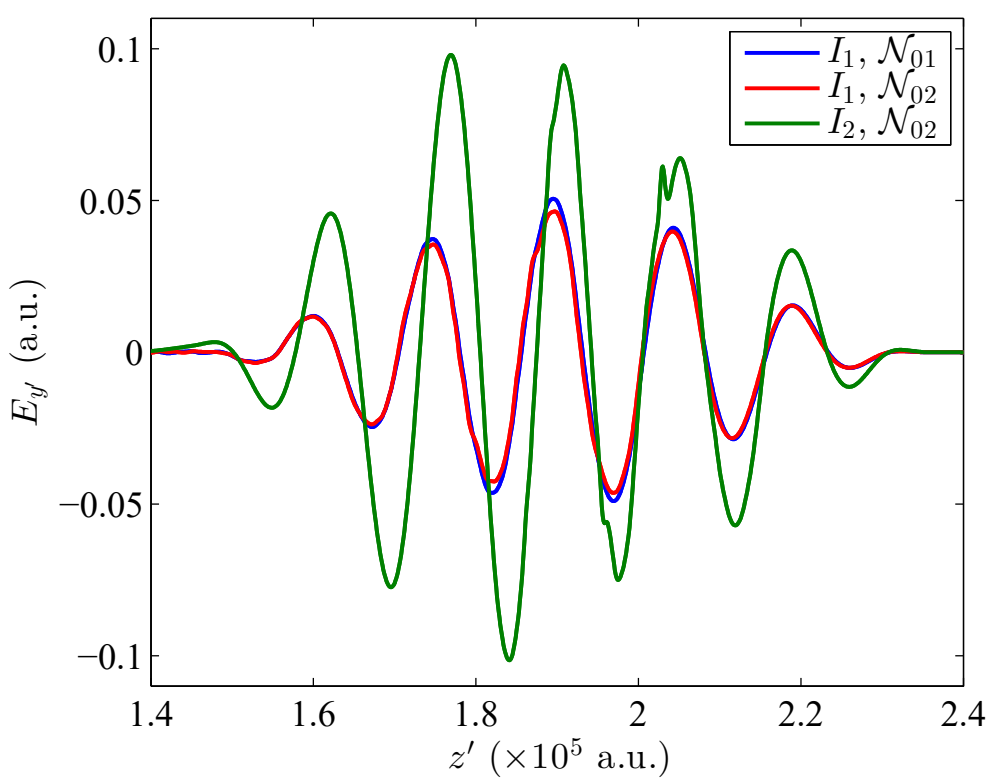

a)

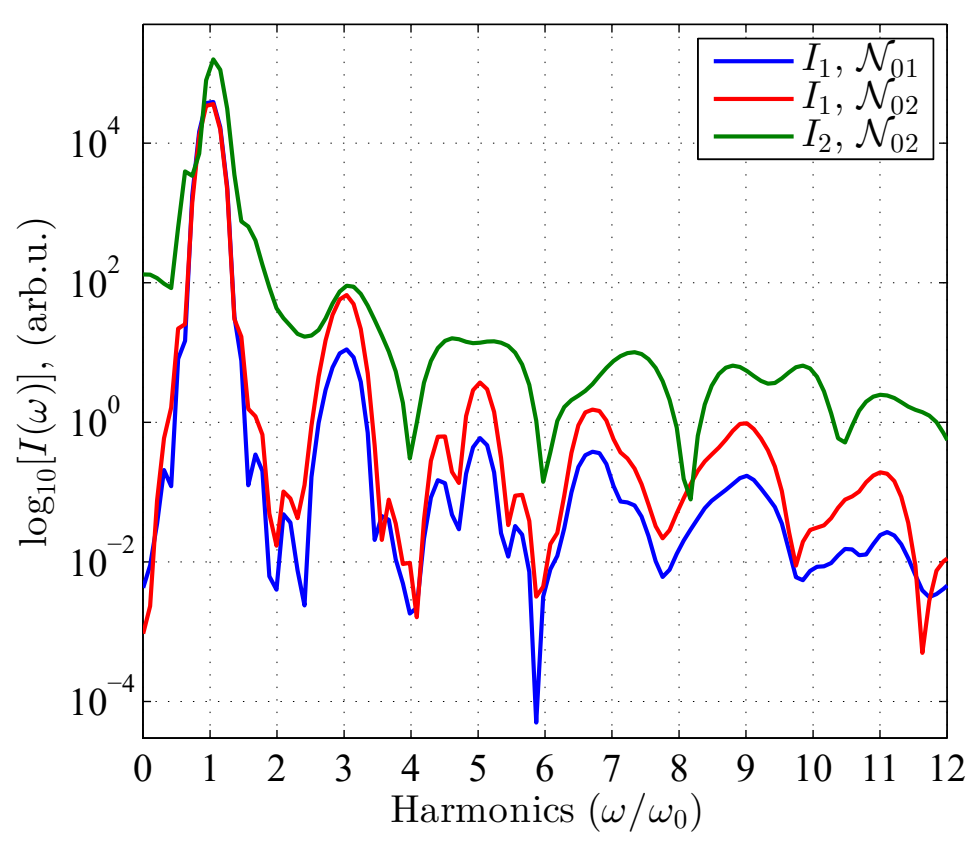

b)

Figure 4.20: Results of the MASP model: a) electric field as a function of space and b) spectral intensity of the laser pulse after propagation through the gas region $L=4 \times 64 \times 100$ a.u. Initial pulse intensities: $I_{1}=10^{14} \mathrm{~W} / \mathrm{cm}^{2}, I_{2}=5 \times 10^{14} \mathrm{~W} / \mathrm{cm}^{2}$, number density of thee gas: $\mathcal{N}_{01}=1.63 \times 10^{-5}$ a.u., $\mathcal{N}_{02}=5.17 \times 10^{-5}$ a.u. 
As we see from Fig. 4.20 a), if we consider pulses of the same initial intensity $I_{1}$, but propagating in the gases of different density, for definiteness $\mathcal{N}_{01}<\mathcal{N}_{02}$, the amplitude of the transmitted electric field occurs a bit lower in the case of a higher density, probably due to stronger ionization losses. However, the generation of the high odd harmonics is more intense for $\mathcal{N}_{02}$, see panel b), as the polarization of the medium is proportional to the gas number density. On the other hand, in the case of initial intensity $I_{2}>I_{1}$ the level of ionization becomes much higher (with respect to initial intensity $I_{1}$ ). As a result, the amplitudes of the high harmonics are relatively smaller.

Including the instantaneous susceptibility coefficient $\chi^{(3)}$ (or even $\chi^{(5)}, \chi^{(7)}$ and so on) as a parameter in the polarization evolution equation (2.36), we are pursuing two objectives: to describe more accurately (i) the EM-field profile and (ii) the spectrum of harmonics. In the following set of figures we report the data computed within the MASP model supplemented by the evolution equations. We start from the length of the gas region $L_{g}=256 \times 100$ a.u., which takes time $T=1051.87$ a.u. to propagate through. We use 3 different values of $\chi^{(3)}$ to test the model. At first glance, even simulations engaging the transport equation with $v_{g}=c$ and $\chi^{(3)}=0$, describe the field profile decently; however, as we are comparing the corrections for nonlinear effects accumulating very slowly, we need to pay attention to the very top parts of the electric field profiles, see Fig. 4.21. Then we notice that in case with initial intensity $I_{1}$, parameter $\chi^{(3)}=10^{-4}$ works better for the both gas densities. In Figs. 4.22 and 4.23, we also report $\ell_{2}$ and $\ell_{\infty}$-norms of all the solutions presented in Figs. 4.21 and the errors computed with respect to the "pure" MASP model. As we observe, in all the figures except $4.22 \mathrm{~b}$ ) and d) ( $\ell_{\infty}$-norms), the blue curves corresponding to $\chi^{(3)}=10^{-4}$ that provides the closest agreement with the results of the "pure" MASP model in conformity with results in Figs. 4.21. 


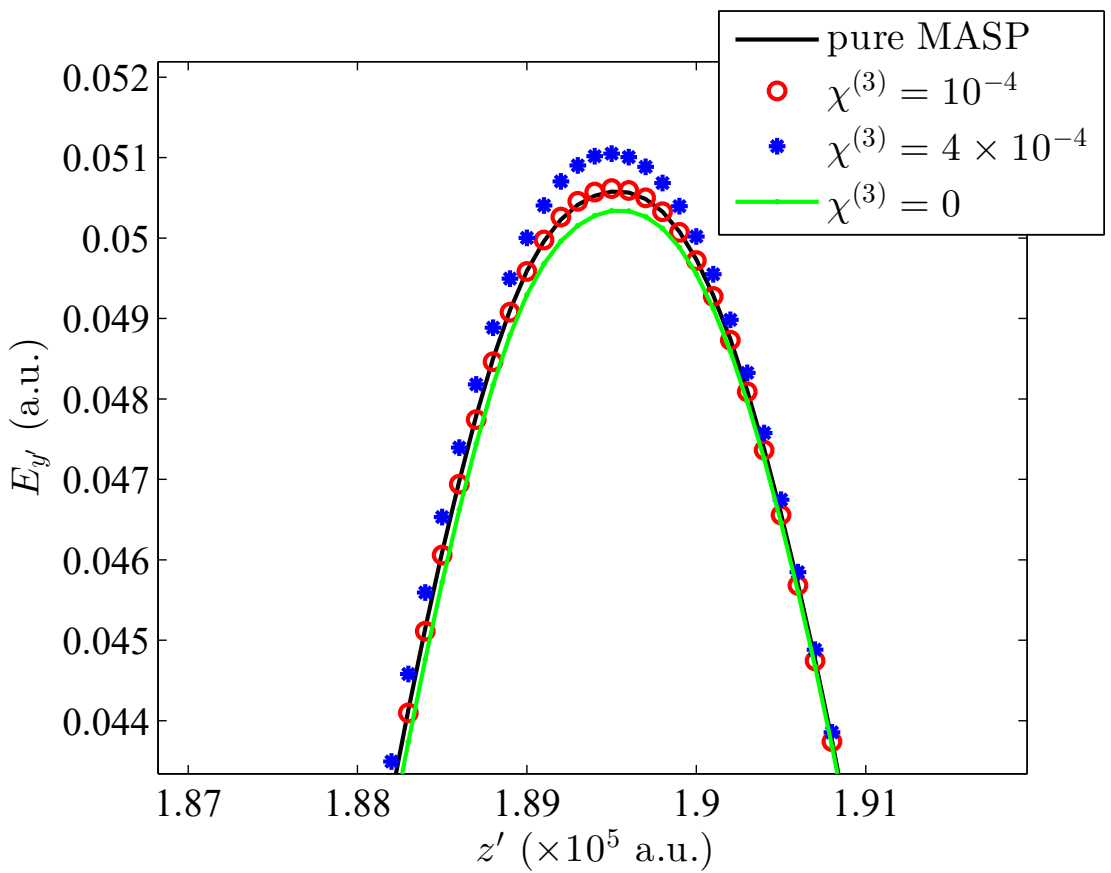

a)

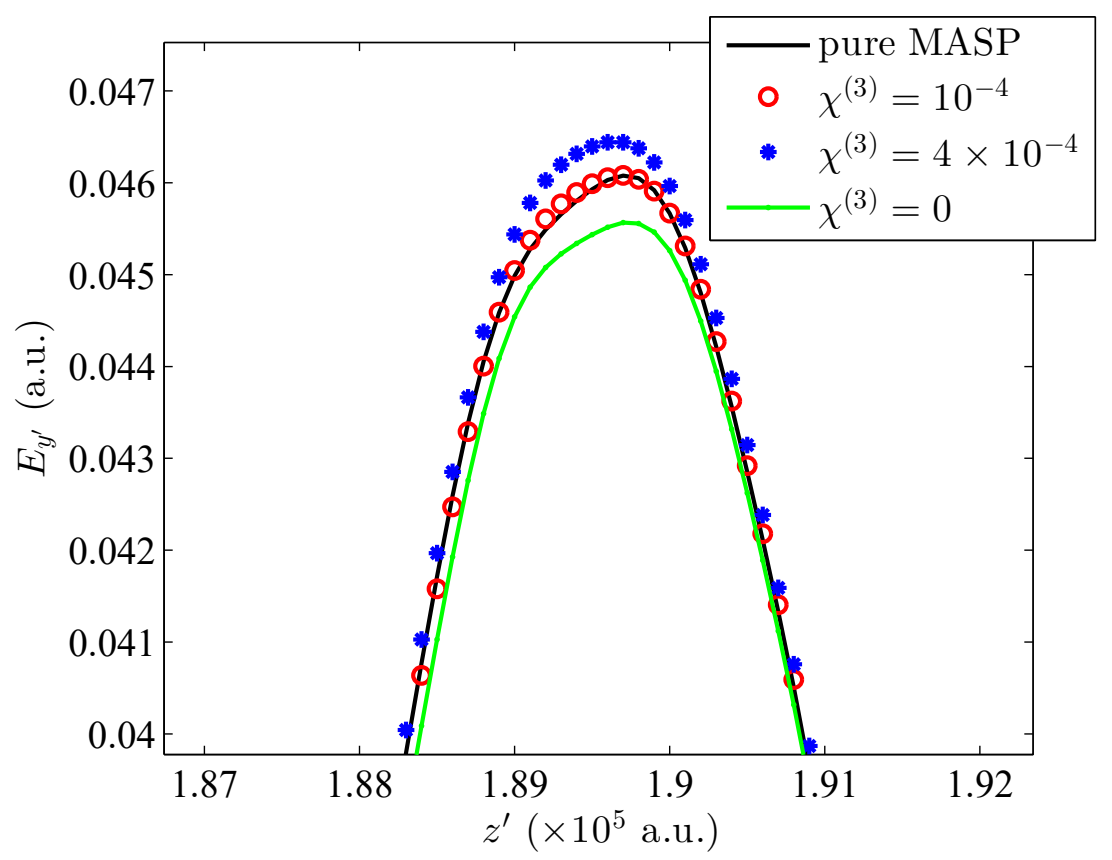

b)

Figure 4.21: Results of the MASP model supplemented by the polarization evolution equation in comparison with the results of the "pure" MASP model: transmitted electric fields for initial intensity $I_{1}=10^{14} \mathrm{~W} / \mathrm{cm}^{2}$ and gas number density a) $\mathcal{N}_{01}=1.63 \times 10^{-5}$ a.u., b) $\mathcal{N}_{02}=5.17 \times 10^{-5}$ a.u. Propagation length in gas $L=256 \times 100$ a.u., which in case of engaging the evolution equation is divided into 4 subdomains each containing 64 cells. Linear instantaneous susceptibility for polarization equation $\chi^{(1)}=1.83 \times 10^{-5}$, while the coefficient $\chi^{(3)}$ is a model parameter, see the legends. 

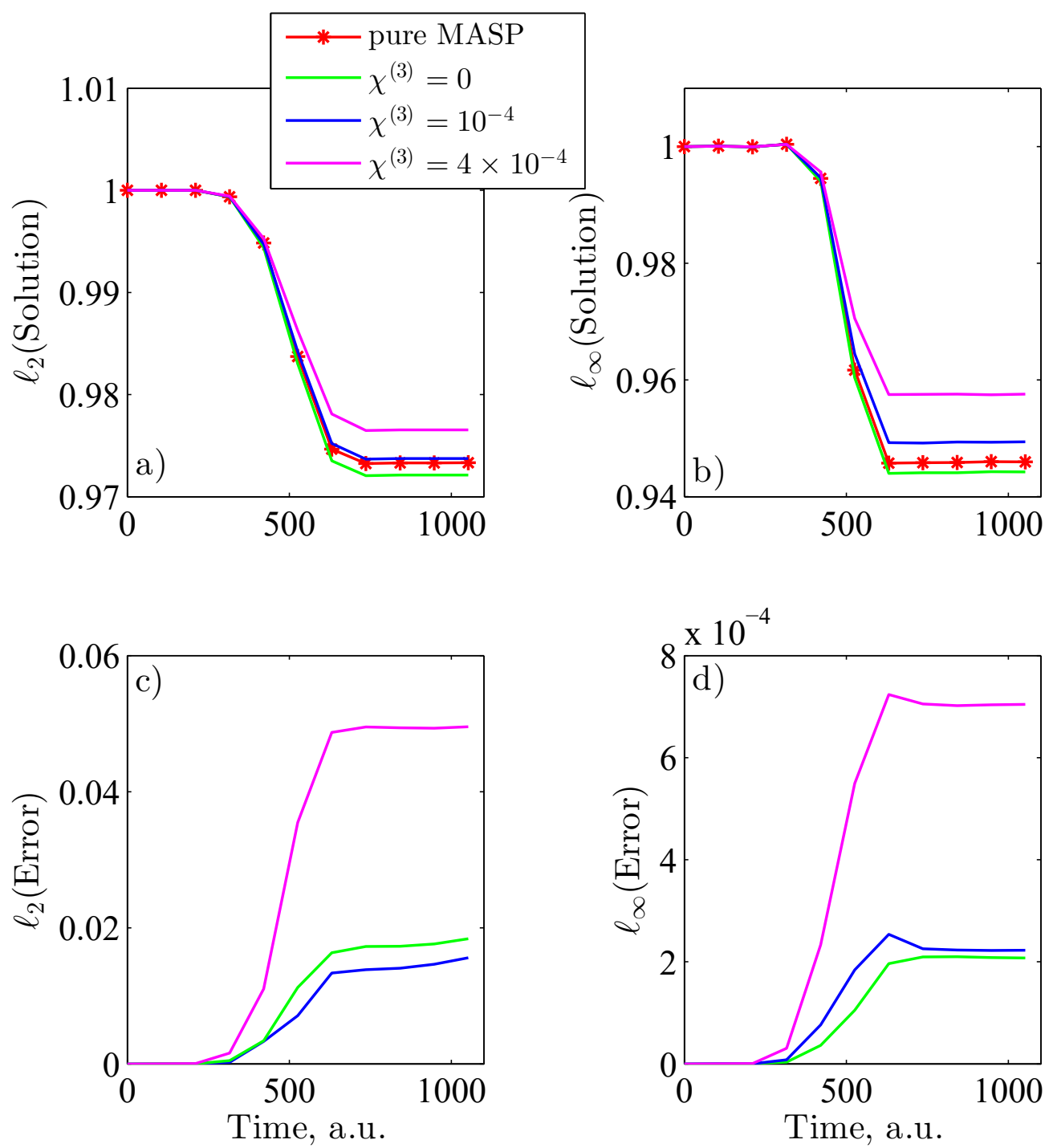

FiguRE 4.22: a) $\ell_{2}$-norms of solutions presented in Fig. $4.21 \mathrm{a}$ ); b) $\ell_{\infty}$-norms of the same solutions; c) $\ell_{2}$-norms of errors of these solutions with respect to the MASP model results; d) $\ell_{\infty}$-norms of errors of these solutions with respect to the MASP model results. 

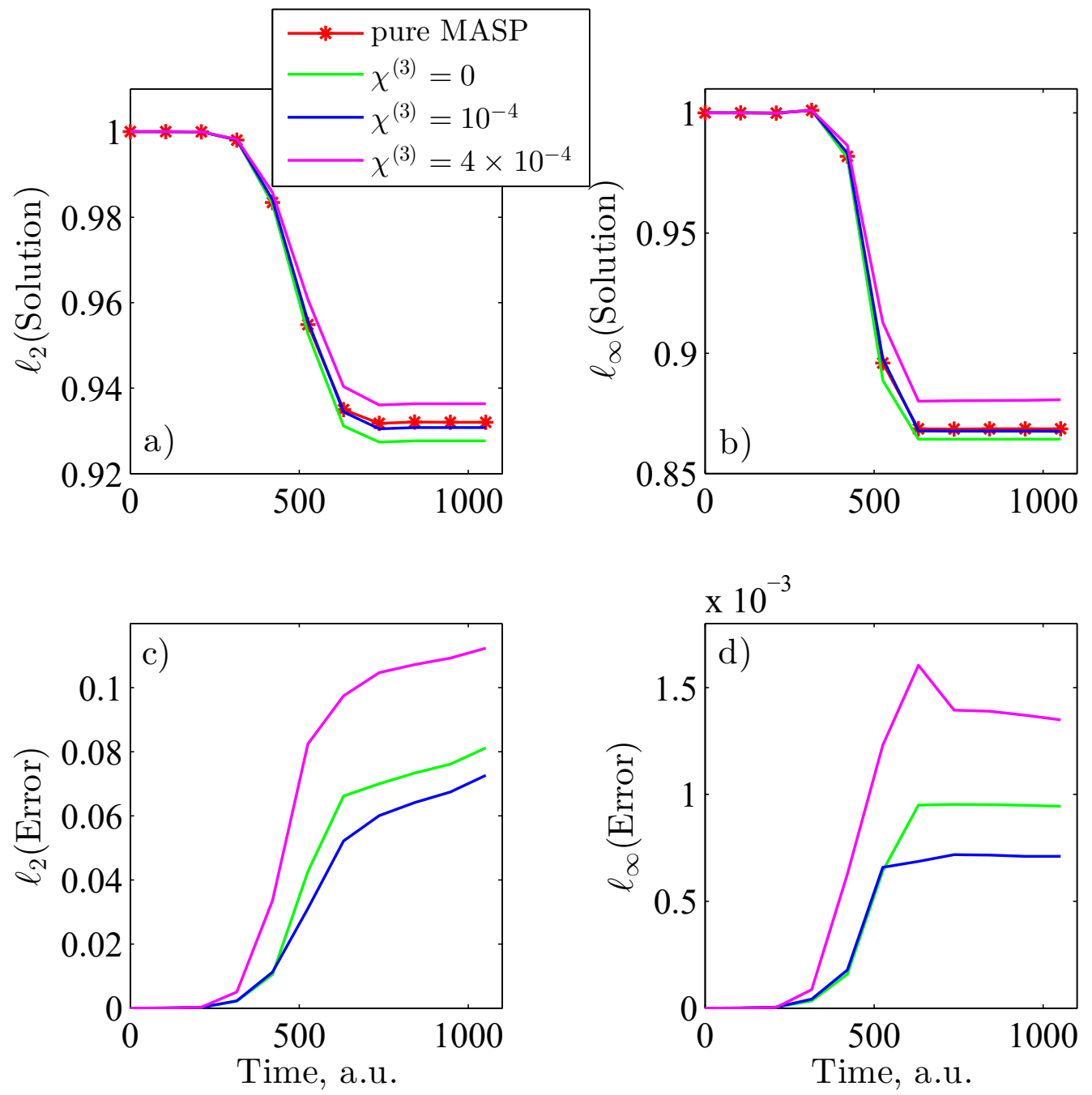

FigURE 4.23 : a) $\ell_{2}$-norms of solutions presented in Fig. $4.21 \mathrm{~b}$ ); b) $\ell_{\infty}$-norms of the same solutions; c) $\ell_{2}$-norms of errors of these solutions with respect to the MASP model results; d) $\ell_{\infty}$-norms of errors of these solutions with respect to the MASP model results. 
The situation changes at higher initial intensities, e.g. $I_{2}=5 \times 10^{14} \mathrm{~W} / \mathrm{cm}^{2}$ that, as we know, results in significant ionization. In this case, introducing instantaneous nonlinear susceptibility does not look like a good idea, see Fig. 4.24 where the part of the electric pulse with maximal differences between all the solutions is represented. The following Fig. 4.25 presents the corresponding $\ell_{2^{-}}$and $\ell_{\infty}$-norms of the solutions and errors. This was natural to expect, as $\chi^{(3)}$ describes the response of bound electrons, whose number is way below than that of free electrons at high intensity. Even so, we still can rely on simulation with the transport equation as it follows from comparison between the black and the green curves in Fig. 4.24, as well as the red and the green curves in Figs. 4.25a), b).

Now we proceed to comparison of the high harmonics spectra, see Fig. 4.26. The simulation parameters for these spectra are exactly the same as for Fig. 4.21. We also report in Figs. 4.27 a), b) the differences between the enriched model and the "pure" MASP model results. From panel a) we see that the selection $\chi^{(3)}=4 \times 10^{-4}$ a.u.

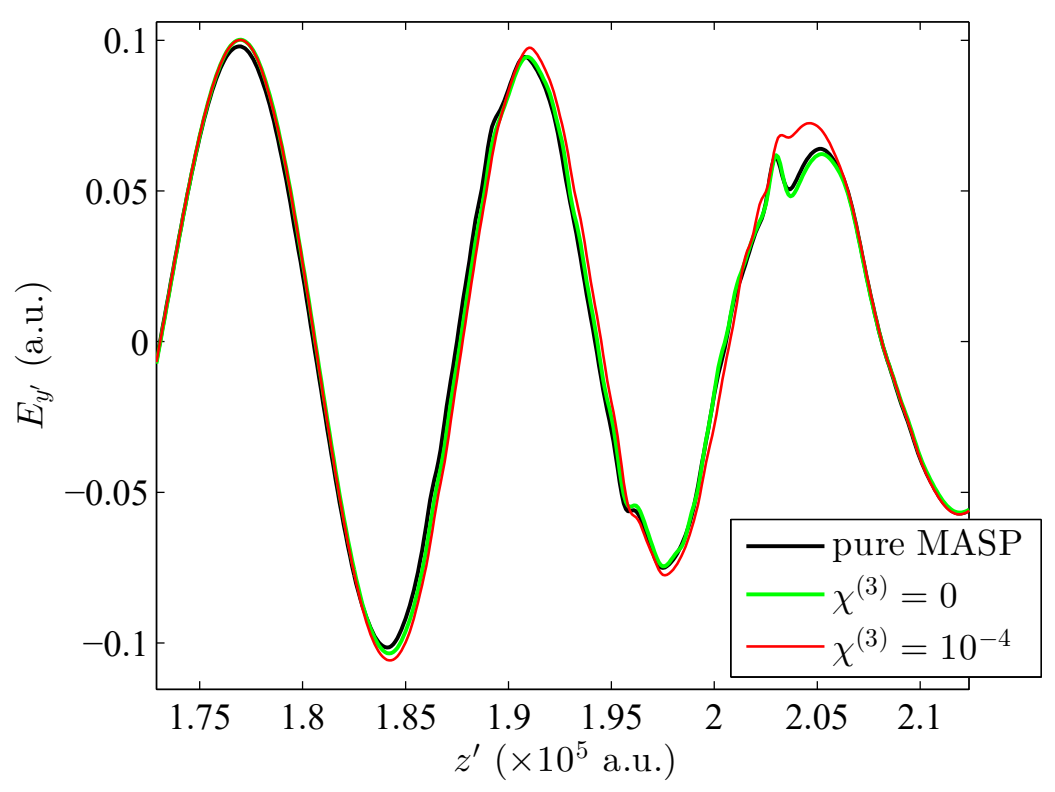

Figure 4.24: Results of the MASP model supplemented by the polarization evolution equation in comparison with the results of the "pure" MASP model: transmitted electric fields for initial intensity $I_{2}=5 \times 10^{14} \mathrm{~W} / \mathrm{cm}^{2}$ and gas number density $\mathcal{N}_{02}=5.17 \times 10^{-5}$ a.u. Path in a gas $L=256 \times 100$ a.u., divided into 4 subdomains each containing 64 cells, $\chi^{(1)}=1.83 \times 10^{-5}, \chi^{(3)}$ is a model parameter, see the legends. 

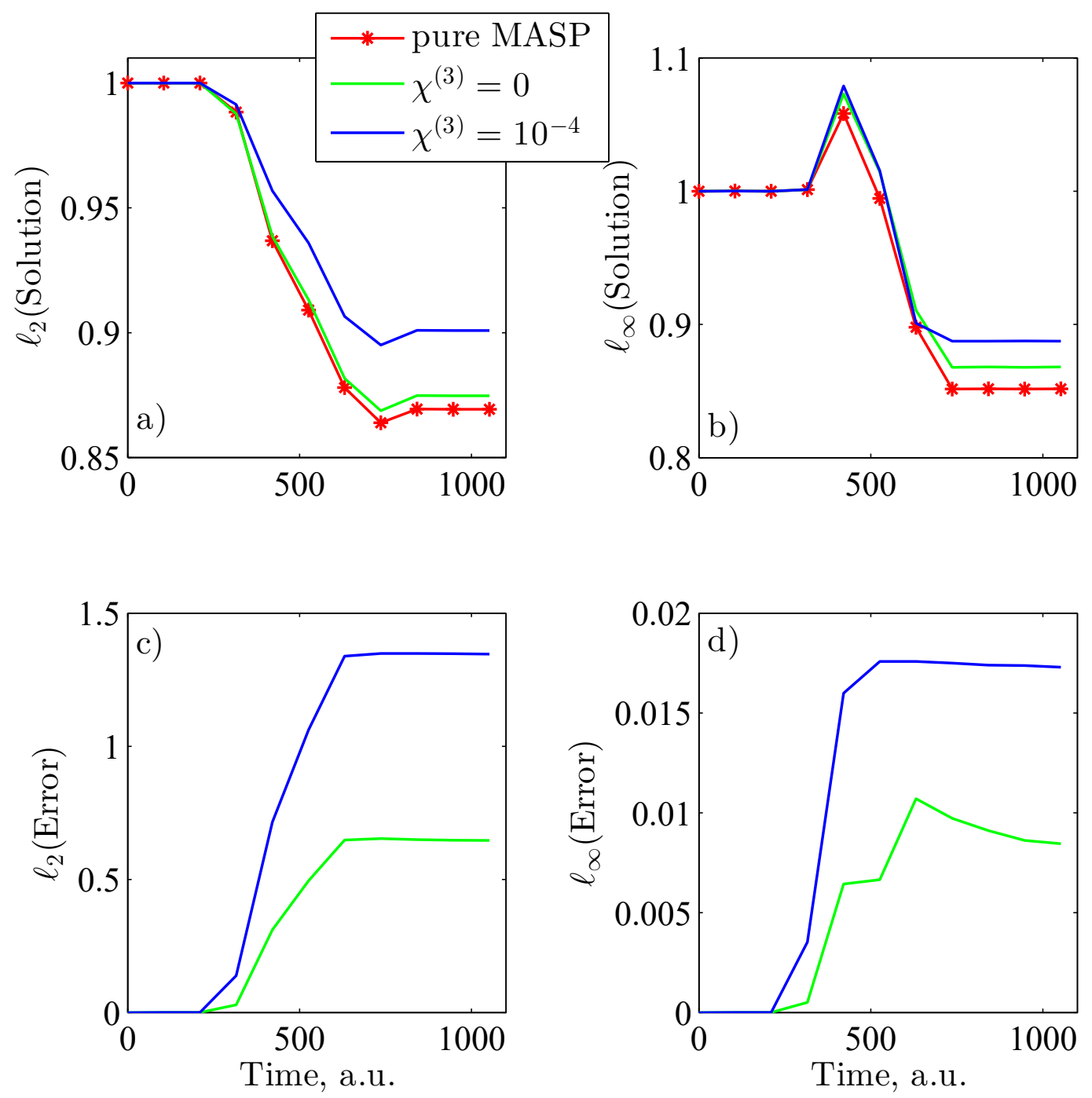

FiguRE 4.25: a) $\ell_{2}$-norms for solutions presented in Fig. 4.24 a); b) $\ell_{\infty}$-norms for the same solutions; c) $\ell_{2}$-norms for errors of these solutions with respect to the MASP model results; d) $\ell_{\infty}$-norms for errors of these solutions with respect to the MASP model results. 
yields less precise results than the two other options. In this case we are overestimating again the response of the bound electrons. Moreover, introducing the coefficient $\chi^{(3)}$ in the polarization equation effect the $3^{r d}$ harmonics generation only, but the description of the THG seems to be adequate even with homogeneous transport equation for polarization.

In Fig. 4.28 we report the high harmonic spectra formed after propagation the pulse with initial intensity $I_{2}=5 \times 10^{14} \mathrm{~W} / \mathrm{cm}^{2}$ through the gas. The first three solutions (according to the legend) correspond to the electric field profiles represented on Fig. 4.24. The $4^{\text {th }}$ solution was obtained under assumption $\mathbf{J}=0$, which as we know is wrong for that intensity. We computed the differences between the solutions (about the odd harmonics only), see Fig. 4.29, so that one can observe the better convergence between the MASP model solution and the MASP supplemented by the transport equation (the green curve). Just as expected, see the solution in red, adding the perturbative term with $\chi^{(3)}$ to the polarization equation is unsuitable in such ionized gas. We also deduce that including the free electron currents in the model is essential, as the solution in blue in Fig. 4.29 is not a good candidate to fit the "pure" MASP model solution.

Regarding the high harmonics spectra simulation at high intensities, the more important factor (than nonlinearity in the polarization equation) is a way of Maxwell's domain decomposition into subdomains, see Figs. 4.30 a), b) corresponding to the propagation length $L=204800$ a.u. The relevant differences between the pure and enriched MASP models are provided in Figs. $4.31 \mathrm{a}, \mathrm{b})$. Say, decomposition into 32 subdomains each containing 64 cells, allows us to simulate accurately up to 35 harmonics, while spending less computing resources: $94 \mathrm{~h} \times 256$ proc vs. $12 \mathrm{~h} \times 32$ proc. Moreover, if we are interested in the first 11 harmonics only, we can use decomposition $8 \times 256$, which takes $12 \mathrm{~h} \times 8$ proc. 


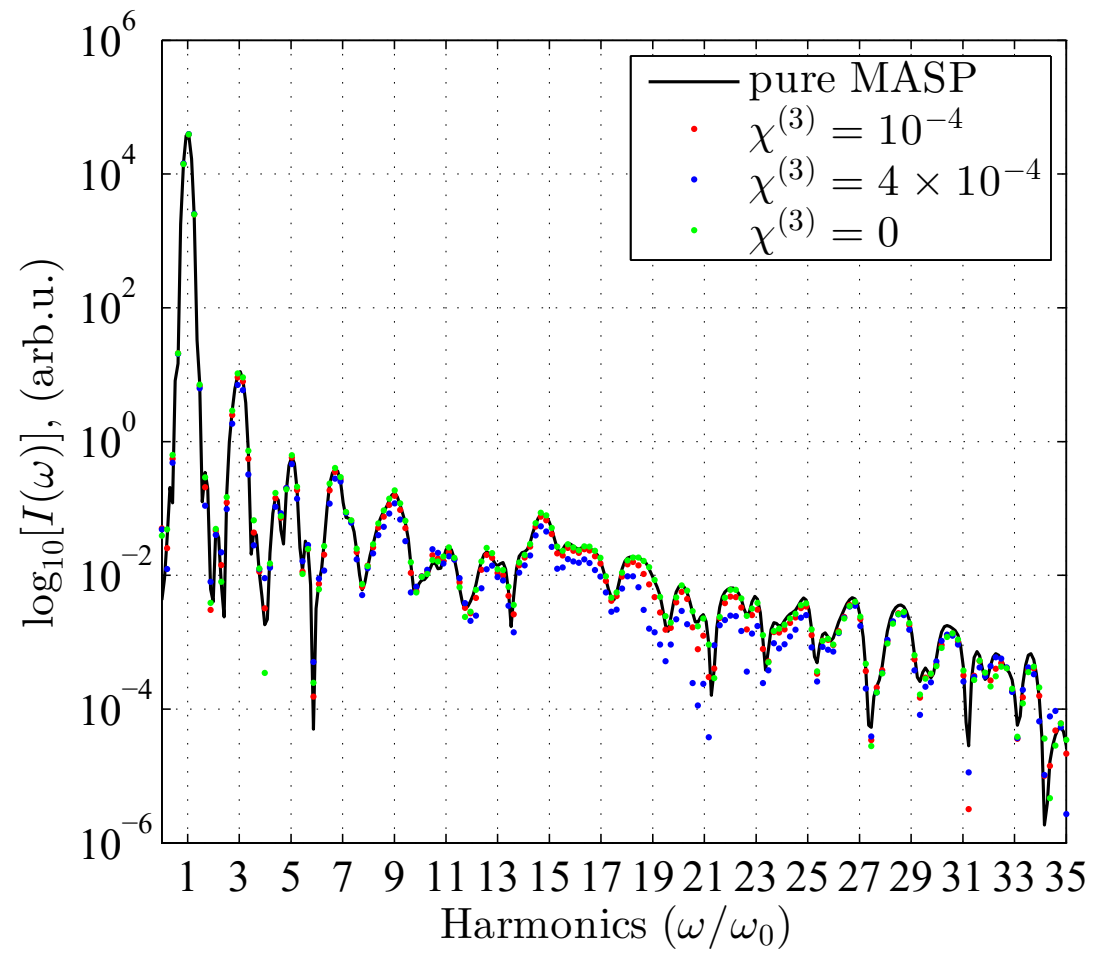

a)

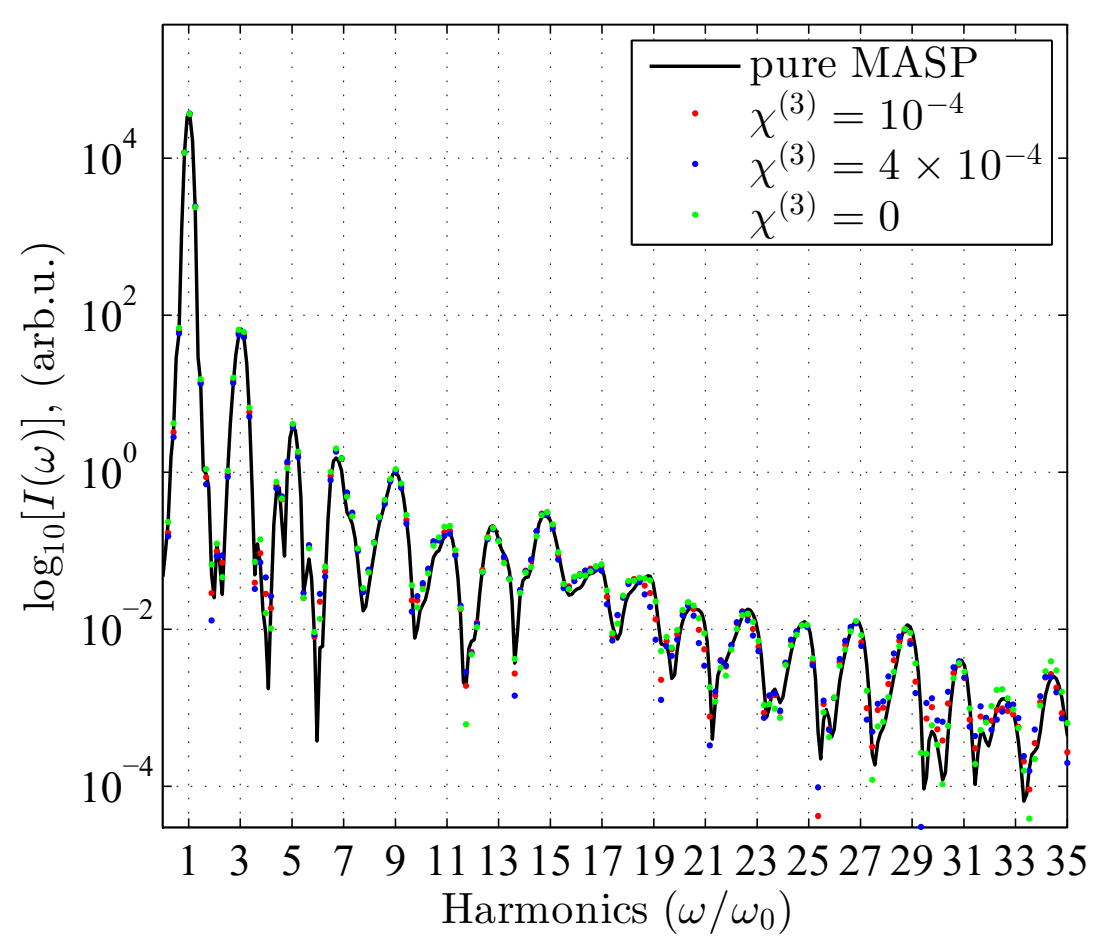

b)

FiguRE 4.26: Spectra of high harmonics. The same parameters as for Fig. 4.21. 


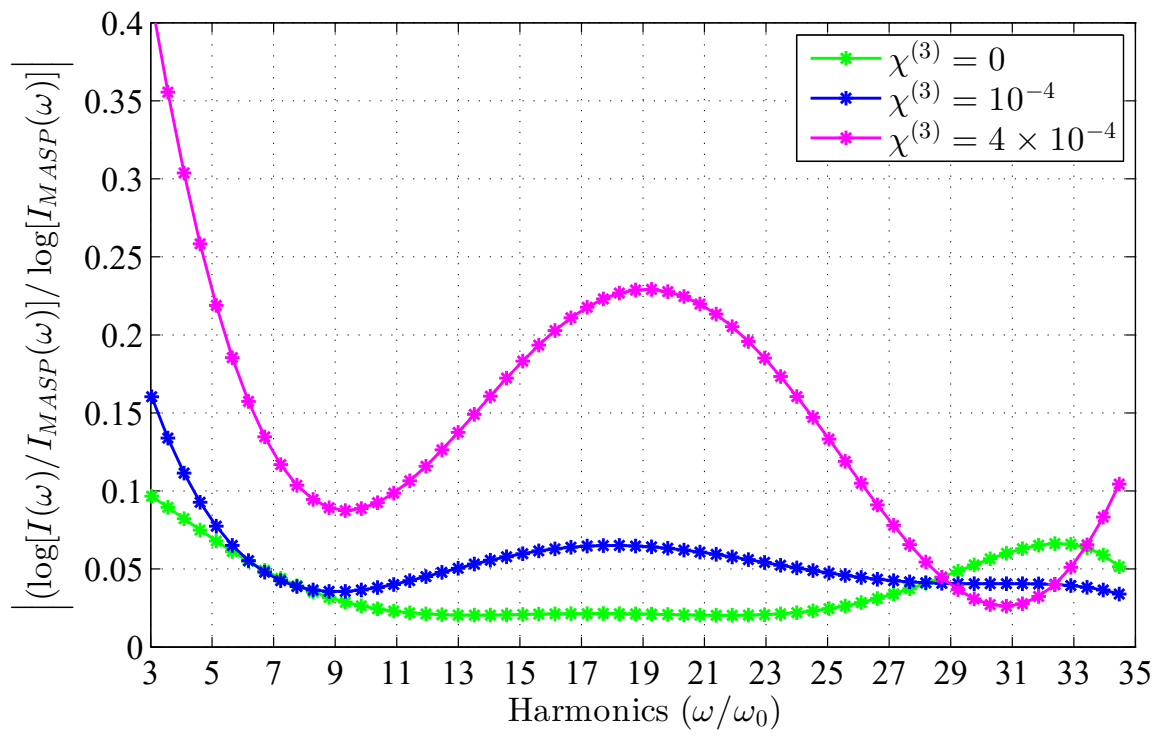

a)

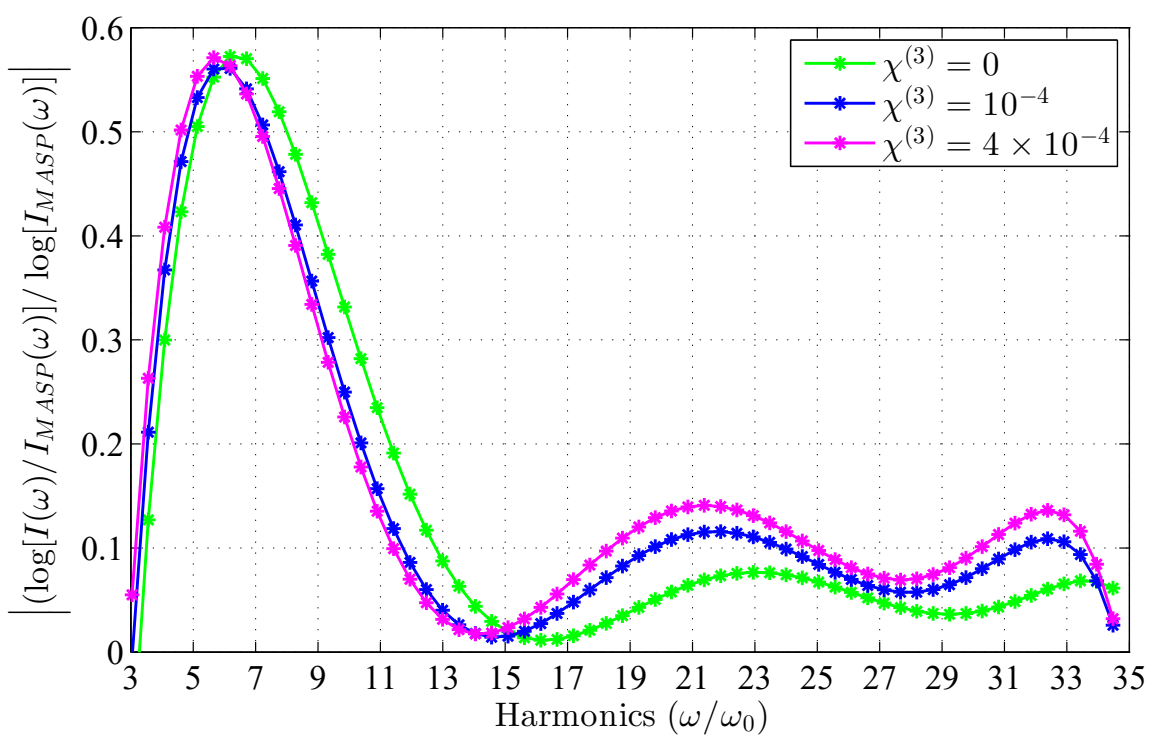

b)

FiguRE 4.27: Difference in spectra of high harmonics presented in Figs. 4.26; the data approximated by polynomial of the $6^{\text {th }}$ degree. 


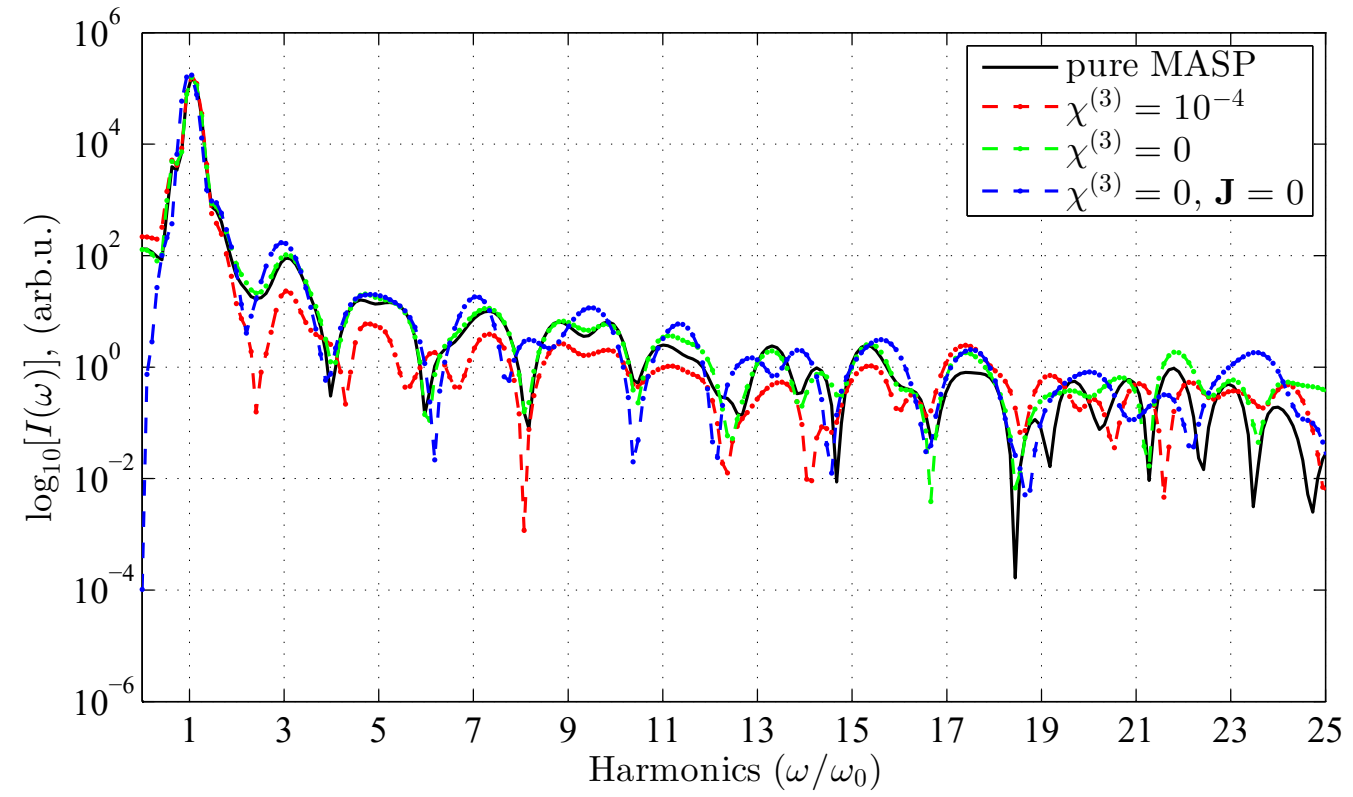

FiguRE 4.28: Spectra of high harmonics. The same parameters as for Fig. 4.24 with additional solution computed with $\mathbf{J}=0$.

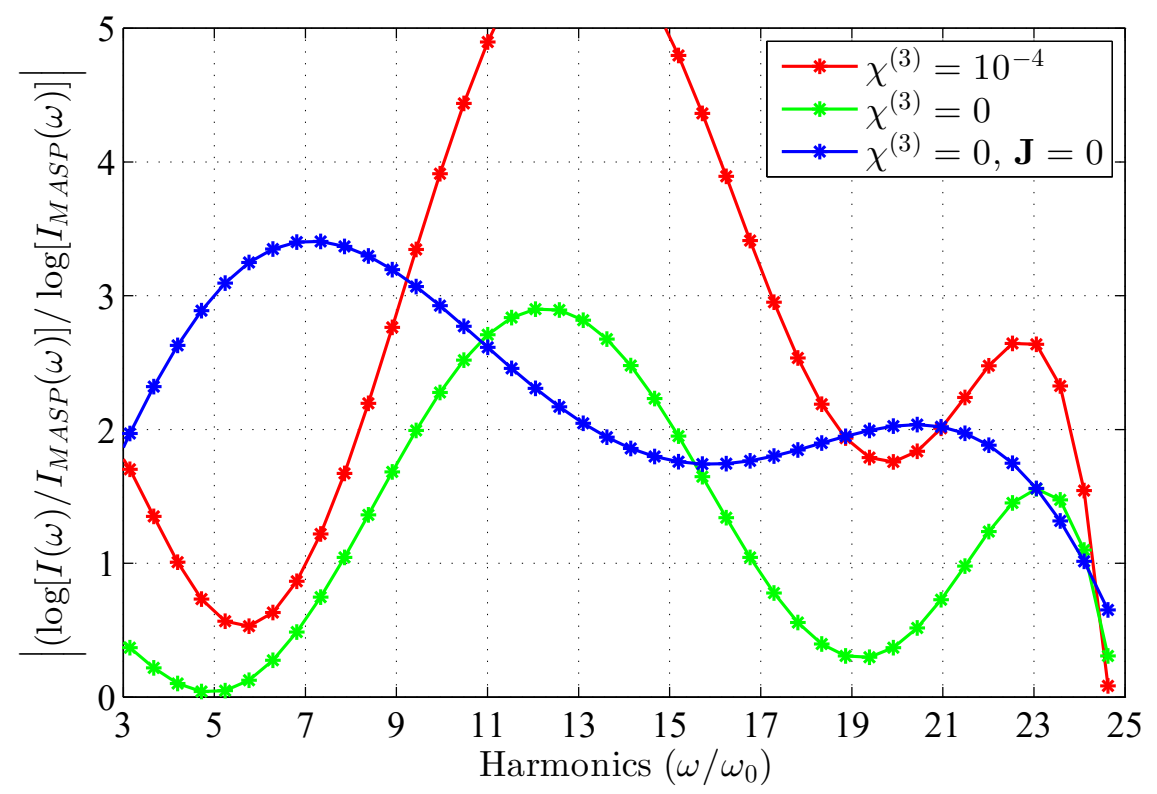

FiguRE 4.29: Difference in spectra of high harmonics presented in Figs. 4.28; the data approximated by polynomial of the $6^{\text {th }}$ degree. 


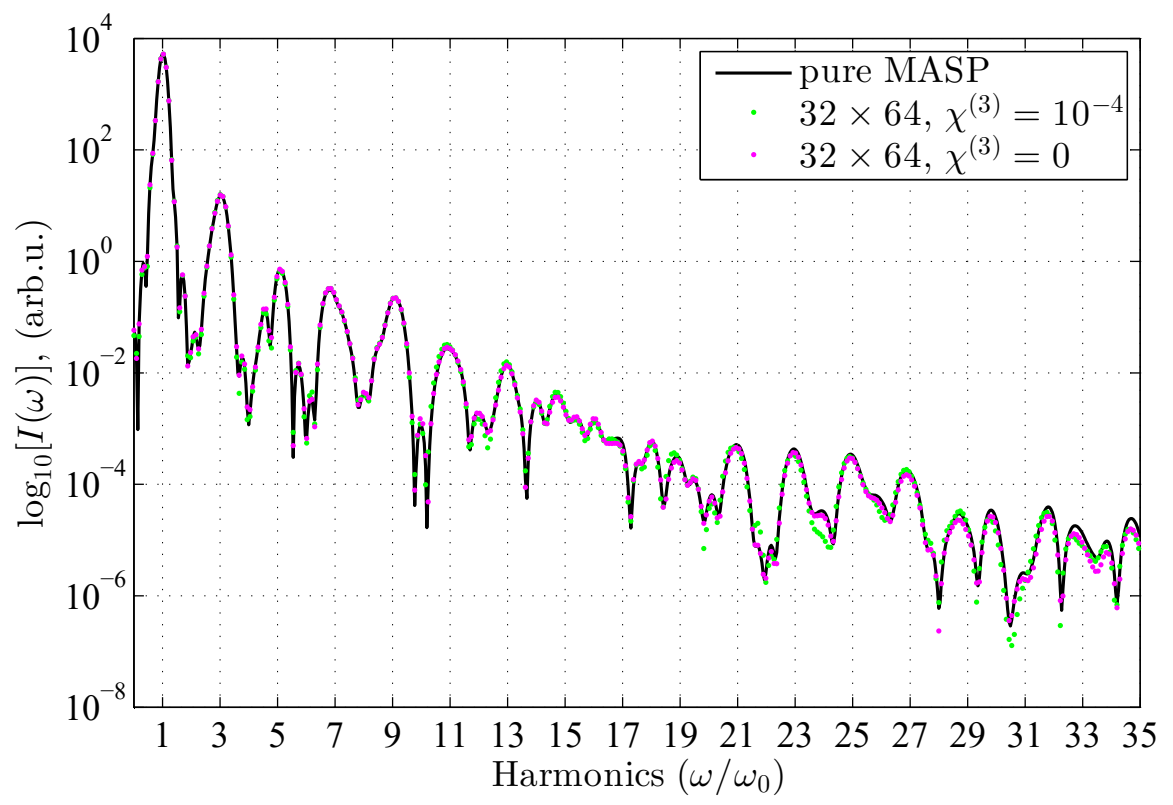

a)

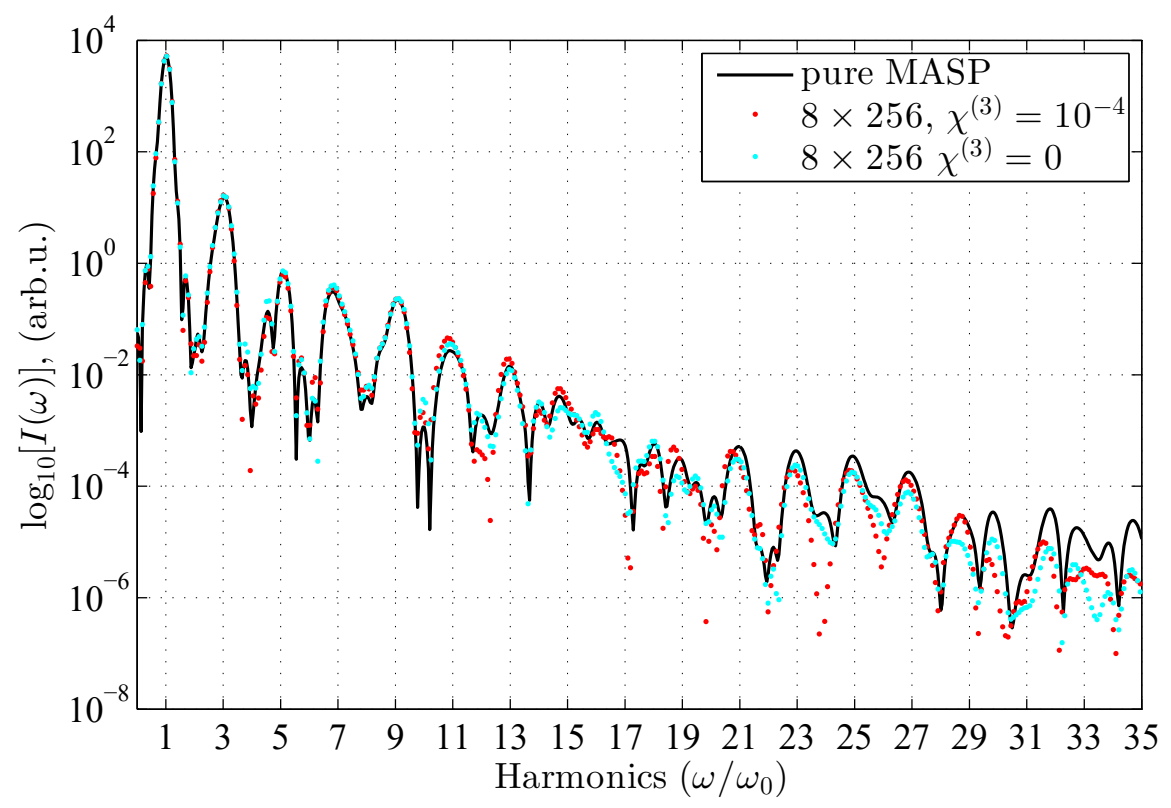

b)

Figure 4.30: Spectra of high harmonics obtained within the "pure" MASP model and using the evolution equation for polarization. The pulse initial intensity $I_{1}=10^{14} \mathrm{~W} / \mathrm{cm}^{2}$, gas density $\mathcal{N}_{01}=1.63 \times 10^{-5}$ a.u., propagation length in a gas $L=204800$ a.u. $(0.01 \mathrm{~mm})$, which is divided into a) 32 subdomains each containing 64 cells and b) 8 sundomains each containing 256 cells. 


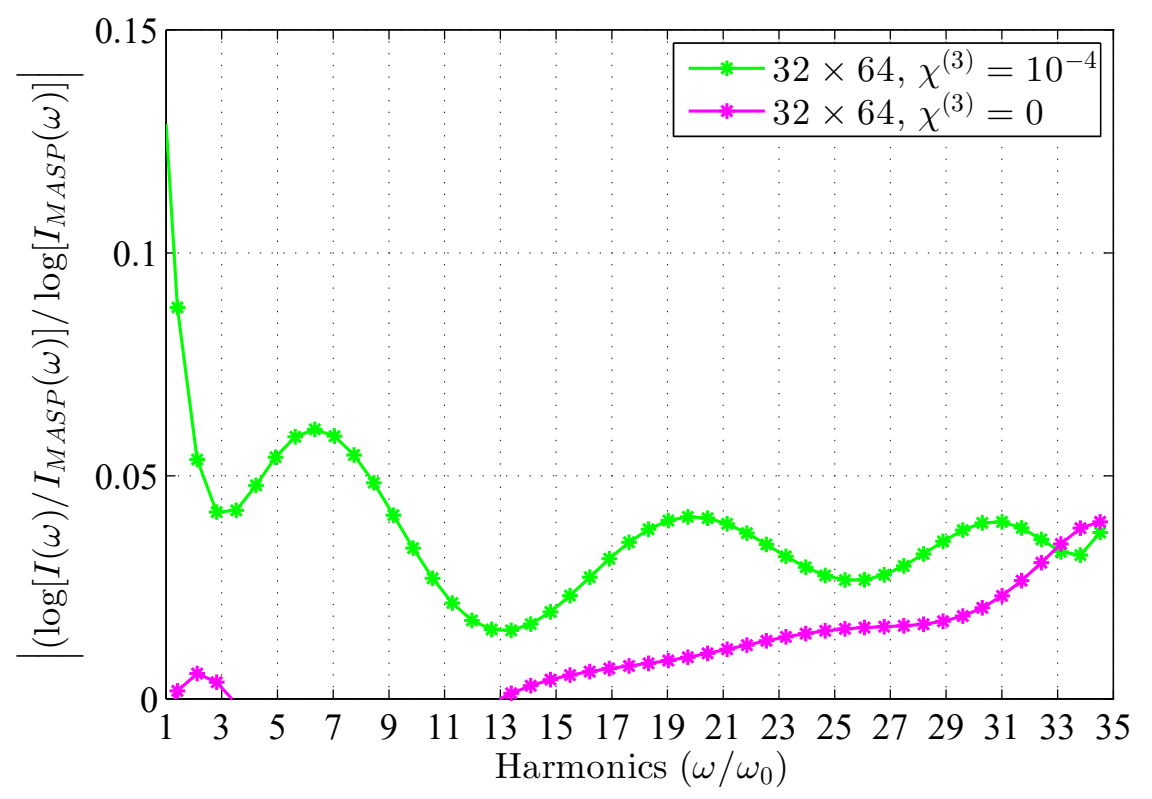

a)

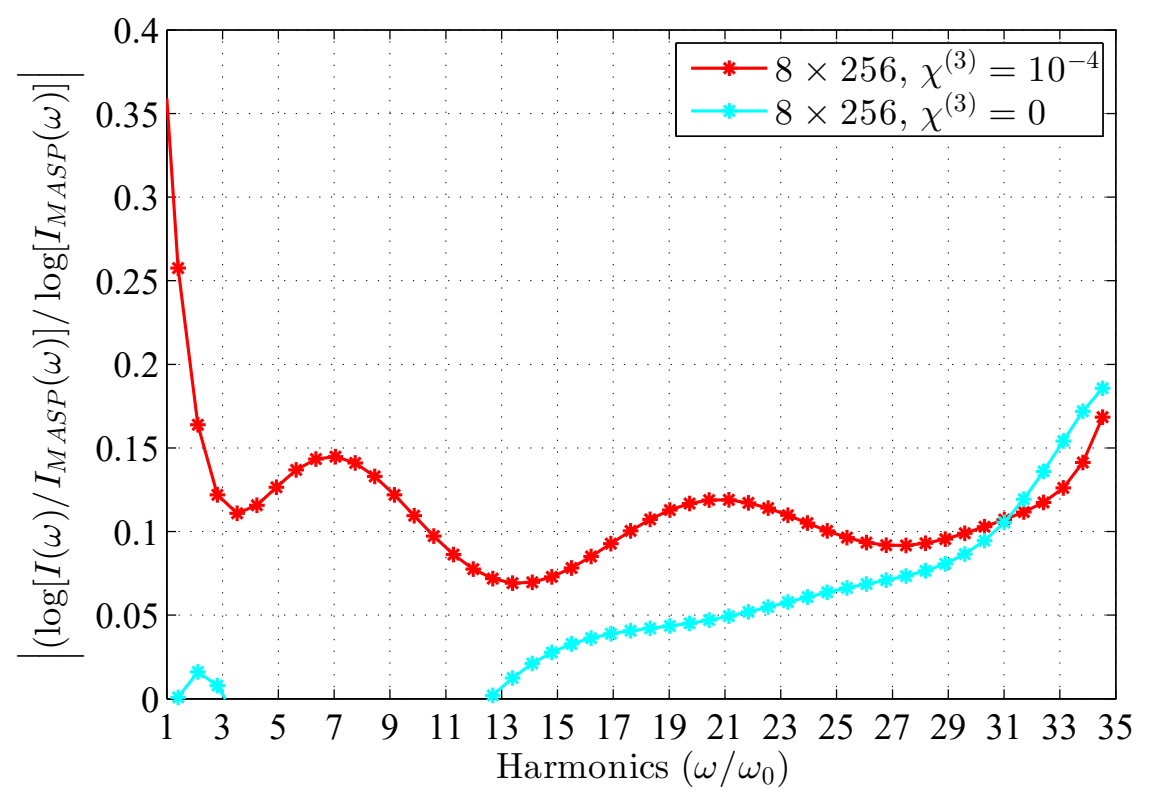

b)

FiguRE 4.31: Difference in spectra of high harmonics presented in Figs. 4.30; the data approximated by polynomial of the $6^{\text {th }}$ degree. 
Let us summarize the findings of Section 4.2.2. We have demonstrated that engaging of the polarization evolution equation makes possible significant reduction of the computational cost for the MASP model. The most universal in such applications is the simplest transport equation, especially if we want to simulate the high harmonics spectrum. The more complicated polarization evolution equation might be useful for modeling of the electric pulse profiles in case when ionization is moderate, i.e. the significant part of electrons are bound. However, if the level of ionization is too high, we should use the transport equation only and take into the account the currents of free electrons while solving the Maxwell's equations. 


\subsection{3d-1d Enriched MASP Model}

In this section we present the very first results obtained within the 3d-1d MASP model enriched by the transport equation for polarization. In order to solve numerically 1d TDSEs, we use the Crank-Nicolson scheme as was described for 2d-TDSEs, see Section 3.3.2; moreover, in the 1d-model we do not need in the ADI method to transform preliminary the $2 \mathrm{~d}$ grid into $1 \mathrm{~d}$, see Fig. 3.4. Such simplification with respect to solving $2 \mathrm{~d}$ TDSE, saves the computational time and is an acceptable choice as soon as we deal with the Gaussian beam under the condition $E_{y^{\prime}} \gg E_{x^{\prime}}, E_{z^{\prime}}$, see (2.13) while $\varepsilon=0$ is set for (2.14) and (2.15). During the beam propagation, which in the current model is governed by 3d-Maxwell's equations, see (3.43), all the EM-field components appear (even those initially equal to zero), so by using the 1d-TDSE we are somewhat reducing the physical reliability of the model. We admit such simplifications for now, as at this stage we intend to develop the model by coupling the polarization equation to the pure MASP model, yet not having in mind comparison with any experimental data for the time being.

To begin with, we consider the envelope of the Gaussian beam (2.13) propagating in the $z^{\prime}$-direction in vacuum, whose evolution is governed by $3 \mathrm{~d}$ Maxwell's equations (3.43), where polarization and currents set to be zeros. We use Yee's scheme (3.45), (3.46) with Mur's absorbing boundary conditions (3.54) to solve the system of MEs. In Fig. 4.32 we present the intensity evaluated through thus computed electric field: $I\left(x^{\prime}, z^{\prime}, t\right)=c E_{y^{\prime}}\left(x^{\prime}, z^{\prime}, t\right)^{2} / 8 \pi$, at different times $t_{n}(n=0, \ldots 7)$. As the wave packet

passes through the focus(waist), we can observe its transversal section become narrower. Fig. 4.33 illustrates the same effect representing the intensity of the beam as a function of the transversal coordinate $x^{\prime}$. 


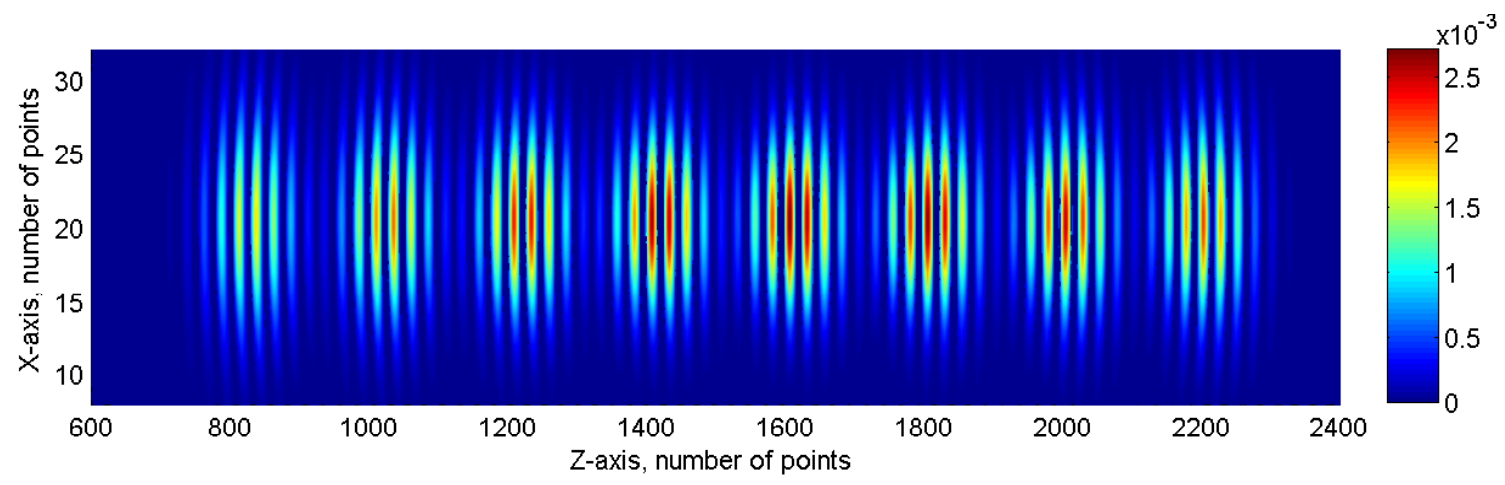

Figure 4.32: Intensity of the envelope of the Gaussian beam calculated for different propagation times: $t_{n}=n \times 438$ a.u. $(n=0 \ldots 7)$ in vacuum, in the $(x, z)$-plane. Maximal initial intensity of the envelope $I=10^{14} \mathrm{~W} / \mathrm{cm}^{2}$, the beam waist $w_{0}=2.1 \mu \mathrm{m}$.

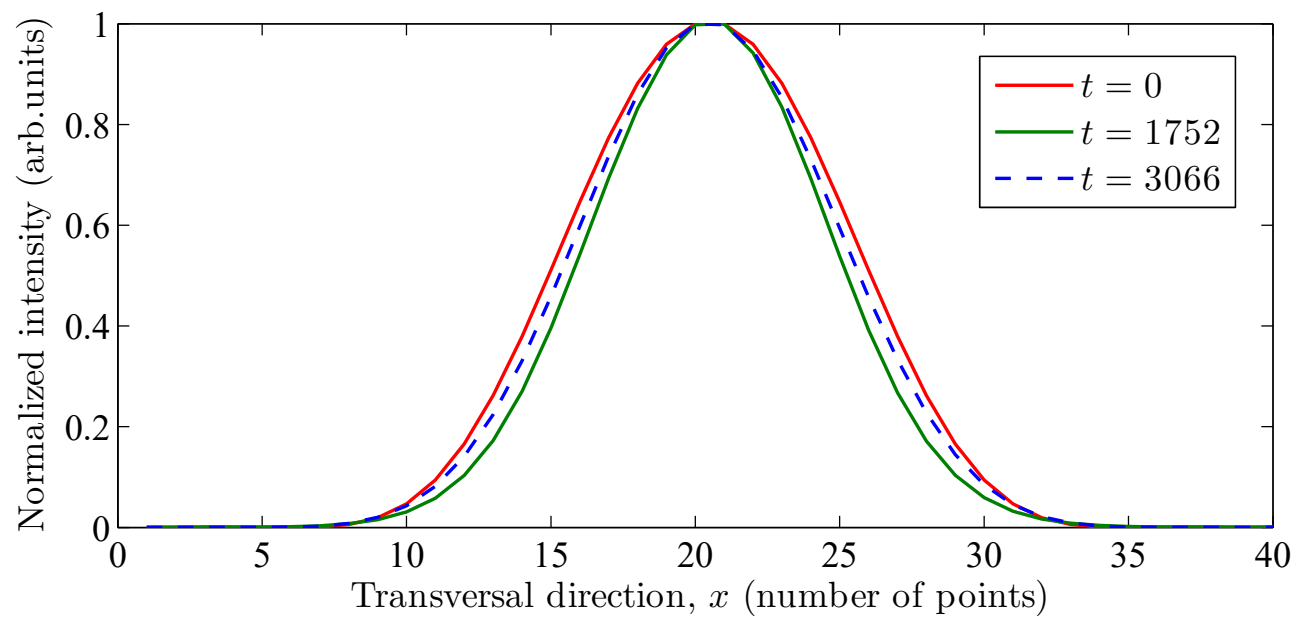

FiguRE 4.33: Pulse width for normalized intensity during propagation in vacuum for 3 moments of time, a.u. Maximal initial intensity of the envelope $I=10^{14} \mathrm{~W} / \mathrm{cm}^{2}$, the beam waist $w_{0}=2.1 \mu \mathrm{m}$.

\subsubsection{Including the Polarization Equation}

We will use the pure MASP model as the reference model, see [82], for the $3 \mathrm{~d}-1 \mathrm{~d}$ model. Figs. 4.34 a), b) compare the data of the pure and enriched models for a propagation length in the gas given by $L=10000$ a.u. $\approx 0.5 \mu \mathrm{m}$. For the gas region we use 20 layers, each of width $\Delta z_{M}^{\prime}=500$ a.u., aligned in the direction of the axis $z^{\prime}$. Each layer has a square section and contains $14 \times 14$ cells with $\Delta x_{M}^{\prime}=\Delta y_{M}^{\prime}=7000$ a.u., see Fig. 3.11. The time step for MEs, $\Delta t_{M}=3.6$ a.u. is evaluated thanks to the CFL-condition (3.56). Space and time steps for the TDSE are $\Delta y_{S}=0.4$ a.u, 


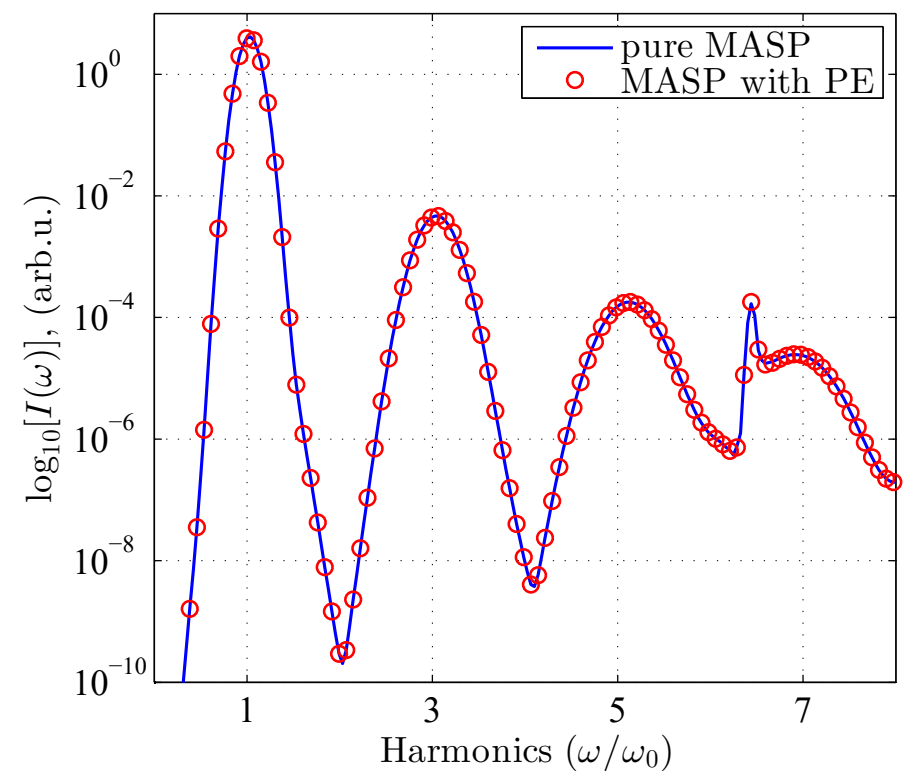

a)

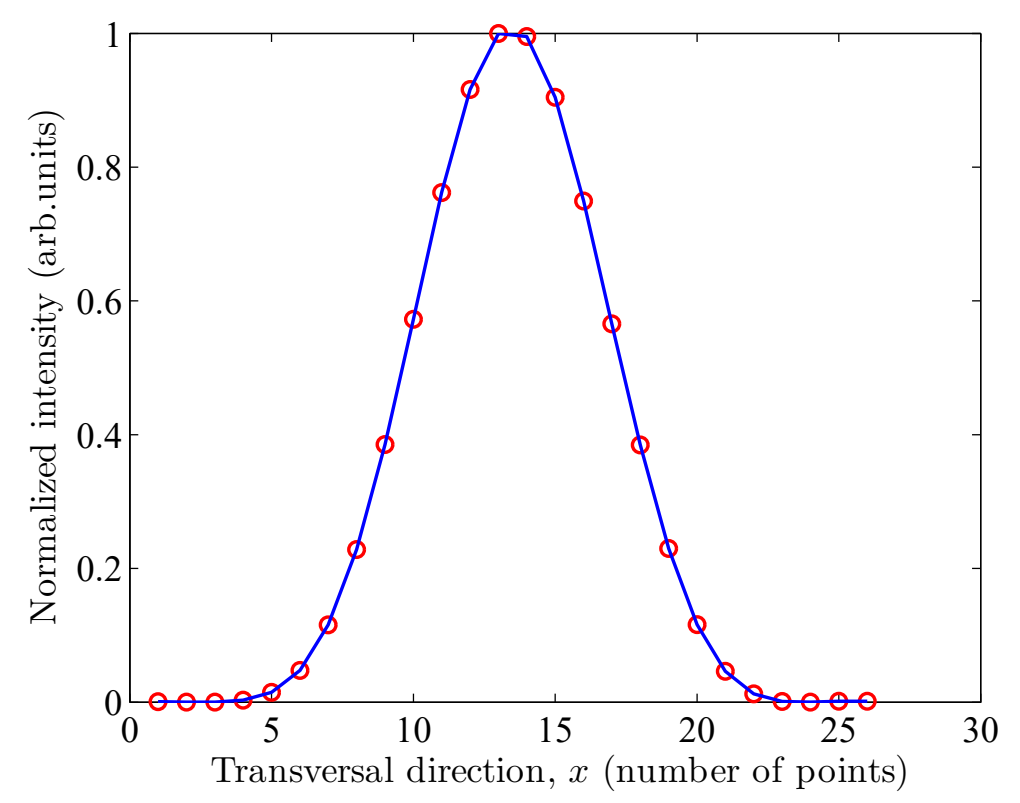

b)

Figure 4.34: (a) Spectra of the low harmonics, and (b) Intensity of the pulse. Resultes computed in the pure MASP and the supplemented MASP models. Parameters are given in the text. 
$\Delta t_{S}=\Delta t_{M} / 30=0.12$ a.u. with the mesh consisting of 100 nodes. In the boundaries of the domain, we used absorbing BCs described in Section 3.3.3. In the full MASP model, the number of layers where the TDSEs are solved is 20, while in the extended model we introduce 2 subdomains, so that we need to solve the TDSEs in the $1^{\text {st }}$ and $11^{\text {th }}$ layers only. For the remaining regions we use the simplest evolution equations (2.17), (2.29) with $v_{g}=c$. Parallelism architecture in given in Fig. 3.12 and described in Section 3.6.3. Fig. 4.34 a) reports the spectra of generated low harmonics. According to the Nyquist-Shannon theorem, the maximum significant harmonic in case of $\Delta_{M} \equiv \Delta z^{\prime}=500$ a.u., $N_{\text {Har }}=15$ (4.4), however, because of very short propagation length, we present only the odd harmonics up to $7^{\text {th }}$. The error with respect to the reference model (circles) does not exceed 3\%. On the panel b) we present the wellmatched intensity profiles as functions of a transversal coordinate.

Finally, Figs. 4.35 a), b) represent solutions of the supplemented 3d-1d MASP model, which were obtained for longer propagation lengths in the $\mathrm{H}_{2}^{+}$-gas. We observe from these computations that in accordance with the theory, see Section 1.3.2,
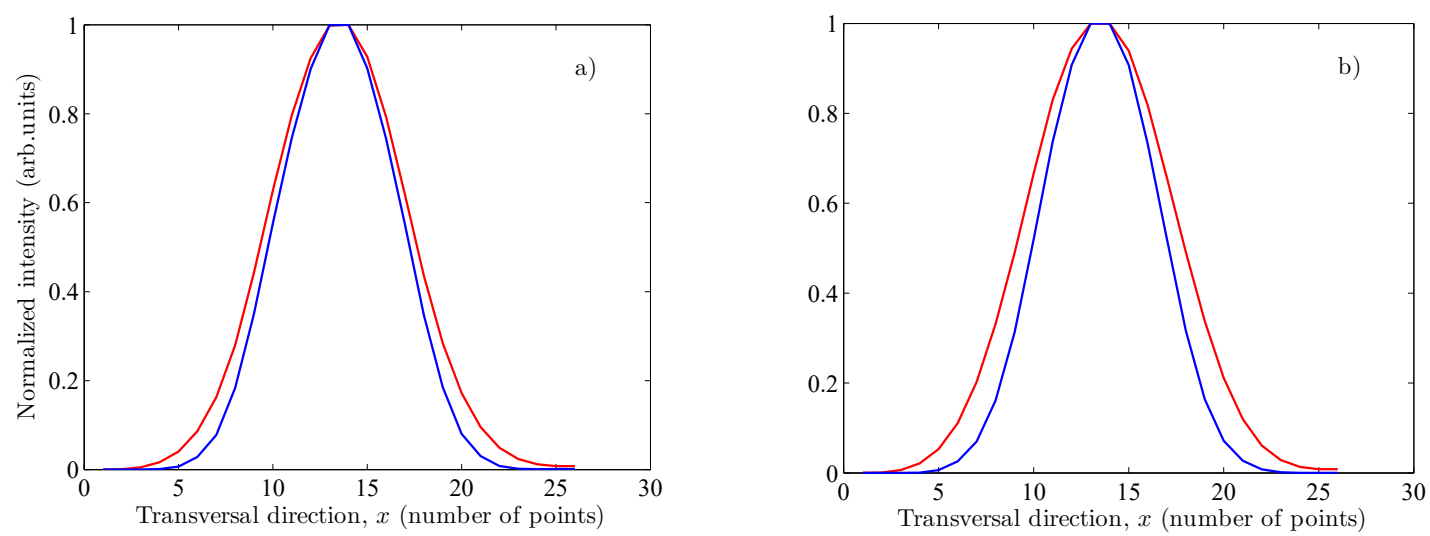

FiguRE 4.35: Normalized intensity profiles of the Gaussian beam with incoming intensity $I=10^{14} \mathrm{~W} / \mathrm{cm}^{2}$ and waist $w_{0}=2.1 \mu \mathrm{m}$, after propagation in vacuum (red curves) and in a gas (blue curves): (a) path in a gas $L_{g}=100,000$ a.u. (10 subdomains each containing 20 cells, $\Delta z^{\prime}{ }_{M}=500$ a.u.), total propagation time $T=1440$ a.u. $=34.56$ fs; (b) $L_{g}=200,000$ a.u. (20 subdomains each containing 20 cells, $\Delta z^{\prime}{ }_{M}=500$ a.u.), total propagation time $T=1800$ a.u. $=43.2 \mathrm{fs}$. 
(i) the pulse propagating in a gas becomes narrower with respect to the case of free propagation in vacuum (self-focusing), and (ii) the longer propagation path in a gas, the more noticeable the difference in the beam thicknesses. The processing time for data, represented on panel (a) is $3 \mathrm{~h}$ when 13 processors are engaged: 3 for MEs and 10 for TDSEs. For the panel (b) the propagation length is longer and the processing time is $3 \mathrm{~h}: 45 \mathrm{~m}$ distributed between 23 processors, as here we used 20 subdomains. Thus, for the examples considered we have a gain around 10-20 processors $\times$ time. Although this gain is not so prominent as in the case of $1 \mathrm{~d}-2 \mathrm{~d}$ models, we can definitely improve it, e.g. by considering the longer propagation lengths divided into subdomains containing more layers. That advantage is essential, especially if we need to use the smaller steps for the Maxwell and Schrödinger equations, e.g. with a prospective of computing the high harmonic spectra or more precise evaluation of filament parameters. 


\section{Chapter 5}

\section{Summary of Findings and Further Directions}

The subject matter of this thesis is the further development of the Maxwell-SchrödingerPlasma (MASP) model developed by E.Lorin, S.Chelkowski and A.Bandrauk [78]. The model is built in a self-consistent "micro-macro" conception, and is very promising in the sense of its applications. Unfortunately, the drawback of the MASP model is the high computational cost. In the thesis we considered new applications of the model, such as CP-pulse propagation in $\mathrm{H}_{2}^{+}$-gas, and proposed several improvements towards reducing of the computational resources necessary for numerical implementation of the approach.

With the aim to decrease the number of computationally expensive TDSEs in the MASP model, the pure model was supplemented by two evolution equations: for polarization and for free electron number density. Now we briefly list what we have already done in this direction and what is yet to be done.

\section{Introducing a mathematical description of the extended MASP model} (Chapter 2) [84].

While in the MASP model, every cell of the MEs domain includes a domain of 
the TDSE, in the extended model the domain of the MEs is divided into $N_{1}$ subdomains, and we have to solve micro TDSEs to compute polarization in the first cell/layer of each subdomain only. Polarization and free electron density in the remaining cells, $N_{2}-1$ per subdomain, are then calculated using the evolution equations that are macroscopic and require less computational resources than TDSEs. As the simplest evolution equations, the corresponding transport equations can be used. We also derived more generic evolution equations.

2. Studying of the well-posedness of the proposed model (Chapter 2).

We have considered the problem of existence and uniqueness for the MASP model on the bounded subdomain with Dirichlet BCs and for the model supplemented by the evolution equations (within one subdomain only).

3. Analysing of the convergence of the numerical schemes that make up the model (Chapter 3).

In numerical implementation of the models we use (i) unconditionally stable Crank-Nicolson scheme (TDSEs), and stable under CFL-condition (ii) LaxFriedrichs scheme (for 1d MEs), (iii) Lax-Wendroff scheme (for 1d MEs and polarization equations), (iv) Yee scheme (for 3d MEs). All the schemes listed, except for the Lax-Friedrichs scheme, have the $2^{\text {nd }}$ order of convergency in time and space.

4. Providing examples that demonstrate effectiveness of the proposed improvements for the model (Chapter 4).

For the first time we managed to simulate generation of the $\mathrm{CP}$ attosecond pulses resulting from propagation of the $\mathrm{CP}$ femtosecond laser pulses through the molecular gas [85]. Recall that initially the effect of high harmonics generation was deemed possible for LP-pulses only. Thus, our results support the theoretical models $[2,7]$ assuming that generation of the high harmonics is also 
possible in the case of the initial CP-pulses.

We presented the results for the spectra and pulse profiles obtained within the 1d-2d pure MASP model and the supplemented MASP model. The results support the idea that inclusion of evolution equations for polarization and free electron density in the model can essentially reduce the number of processors and/or processing times, and storage memory, without noticeable reduction in accuracy. We showed that when ionization is not very strong, the use of evolution equations with perturbative nonlinearity is justified, whereas under conditions of a high ionization, it is especially important to take into account the currents of free electrons.

5. Application to filament propagation. Certainly, there are many interesting and important cases left outside of our numerical simulations. One of them is studying of filament propagation under the paraxial approximation, see Section 2.3.3. Another tool for modeling a filament is provided by $3 \mathrm{~d}-1 \mathrm{~d} / 2 \mathrm{~d} / 3 \mathrm{~d}$ MASP models. We demonstrated in Section 4.3 that using the extended MASP model can be helpful in simulation of the Gaussian beam propagation in the $\mathrm{H}_{2}^{+}$-gas with less computational costs (still with respect to the pure MASP model). However, our results were performed within $3 \mathrm{~d}-1 \mathrm{~d}$ model and for short lengths only, so they still need to be improved.

A promising opportunity to speed the processing of the MASP model is provided by analytical nonperturbative solutions to TDSE, which have been considered in $[37,108]$. If we can find similar solutions for gas molecules of interest, those will greatly simplify and speed up the computations. 


\section{Appendices}




\section{Appendix A}

\section{Atomic units}

Throughout the thesis, the equations, as well as the numerical calculations are written in Hartree atomic units (a.u.) [34]. For this system four fundamental physical constants, reduced Planck's constant, electron mass, elementary charge, and Coulomb's constant, set equal to 1 :

$$
\hbar=m_{e}=e=k_{e}\left(=1 /\left(4 \pi \varepsilon_{0}\right)\right)=1 .
$$

Using another fundamental physical constant, the fine-structure constant, $\alpha=$ $=(137.035999074(44))^{-1}$, which reads in the International System of Units (SI) as $\alpha=e^{2} /\left(4 \pi \varepsilon_{0} \hbar c\right)$, one can express the speed of light as $c=\alpha^{-1} \approx 137.036$ a.u.

It is useful to notice that in atomic units, the Bohr radius plays the role of the length unit: $a_{0}=4 \pi \varepsilon_{0} \hbar^{2} /\left(m_{e} e^{2}\right)=1$ a.u. $\approx 0.0529 \mathrm{~nm}$. For example, in our computations the molecular density $3.5 \times 10^{20} \mathrm{~mol} / \mathrm{cm}^{3}$ corresponds to $5.17 \times 10^{-5} a_{0}^{-3}$.

We also need to express the frequency in a.u. through the wave length in $\mathrm{nm}$ :

$$
\omega_{\text {a.u. }}=\frac{2 \pi c_{\text {a.u. }}}{\lambda_{\text {a.u. }}}=\frac{2 \pi}{\alpha} \frac{a_{0}}{\lambda} \approx \frac{45.55}{\lambda(\mathrm{nm})} .
$$

Namely, for calculations where $\lambda=800 \mathrm{~nm}$, one gets $\omega \simeq 0.057$ a.u. 
In addition, the energy of the ground state of the hydrogen atom (Hartree energy) plays the role of the energy unit: $E_{h}=m_{e} e^{4} /\left(4 \pi \varepsilon_{0} \hbar^{2}\right) \approx 4.3597 \times 10^{-18} \mathrm{~J} \approx 27.21 \mathrm{eV}$. Then, the time unit is defined as $t_{h}=\hbar / E_{h} \approx 2.419 \times 10^{-17} \mathrm{~s} \approx 24$ as.

The unit of the electric field intensity $E_{\text {at }}=e^{2} / a_{0}$ corresponds to $5.17 \times 10^{9} \mathrm{~V} / \mathrm{cm}$ in SI. The unit of intensity in a.u. is $I=\frac{\varepsilon_{0} c E_{\mathrm{at}}^{2}}{2}=3.5 \times 10^{16} \mathrm{~W} / \mathrm{cm}^{2}$ in SI. 


\section{Appendix B}

\section{Basic Facts About the Nonlinear}

\section{Schrödinger Equation}

We are reviewing here some properties of the NLSE for the complex function $\psi(\mathbf{x}, t)$ : $\left(\mathbb{R}^{n}, \mathbb{R}_{+}\right) \rightarrow \mathbb{C}, n=\{1,2,3\}$, on the assumption of attractive nonlinearity, $\kappa>0$ :

$$
\mathrm{i} \partial_{t} \psi+\triangle \psi+\kappa|\psi|^{2} \psi=0
$$

since this form corresponds to the relevant applications, see (1.85) and (1.108). The amplitudes of the waves described by the NLSE are assumed to be small, so we consider the nonlinear effects accumulated with time and propagation length [114]. A soliton solution (1.87) to $1 \mathrm{~d}\left(\triangle=\partial_{x x}\right)$ NLSE (1.85) corresponds to the case of propagation of an almost monochromatic envelope in dispersive medium with weak nonlinearity [92]. Indeed, consider 1d quasi-harmonic wave [104]:

$$
\psi(x, t)=a(x, t) e^{\mathrm{i}\left(k_{0} x-\omega_{0} t\right)}
$$

with slowly varying amplitude: $\left|\partial_{t} a\right| \ll\left|-i \omega_{0} a\right|,\left|\partial_{x} a\right| \ll\left|i k_{0} a\right|$, so that its dispersion relation is in form $\omega=\omega\left(k,|a|^{2}\right)$. For narrow spectrum, $\left|k-k_{0}\right| \ll k_{0}$, and weak 
nonlinearity, $|a|^{2} \ll k_{0}^{2}$, we expand $\omega$ in a Taylor's series about $\left(k_{0}, 0\right)$, that is:

$$
\omega=\omega_{0}+\left.\partial_{k} \omega\right|_{\left(k_{0}, 0\right)}\left(k-k_{0}\right)+\left.\frac{1}{2} \partial_{k}^{2} \omega\right|_{\left(k_{0}, 0\right)}\left(k-k_{0}\right)^{2}+\left.\partial_{|a|^{2}} \omega\right|_{\left(k_{0}, 0\right)}|a|^{2}+\ldots
$$

Then we introduce the frequency and the wavenumber of the envelope:

$$
\Omega=\omega-\omega_{0}, \quad K=k-k_{0},
$$

so that $\partial_{t} a=-i \Omega a$ and $\partial_{x} a=-i K a$ and thus

$$
i\left(\partial_{t} a+v_{g} \partial_{x} a\right)+\frac{\omega_{0}^{\prime \prime}}{2} \partial_{x}^{2} a+\beta|a|^{2} a=0
$$

where the group velocity of the envelope is denoted as $v_{g}=\left.\partial_{k} \omega\right|_{\left(k_{0}, 0\right)}$, the dispersion of the wave $\omega_{0}^{\prime \prime}=\left.\partial_{k}^{2} \omega\right|_{\left(k_{0}, 0\right)}$, and a coefficient $\beta=\left.\partial_{|a|^{2}} \omega\right|_{\left(k_{0}, 0\right)}$ characterizes the degree of nonlinearity. In the frame of reference moving with the group velocity, we indeed obtain the NLSE from (B.5). Note that a more rigorous derivation of NLSE $[92,114]$ is based on the multiple-scale analysis [19] with slow times $T_{1}=\varepsilon t, T_{2}=\varepsilon^{2} t$ and scale $X=\varepsilon x$. Then the equation for slow amplitude $a$ reads

$$
\partial_{T_{2}} a=\frac{i \omega^{\prime \prime}}{2} \partial_{\xi}^{2} a+i \beta|a|^{2} a
$$

where $\xi=X-v_{g} T_{1}$, appears as a condition for canceling of secular terms in the first order in $\varepsilon[92,114]$.

As is well known, the presence of the nonlinearity in the wave equation can lead to the self-steepening and formation of the shock waves [121], see Fig. B.1. In nonlinear and dispersive medium, stable soliton solution for the NLSE is possible, but in 1d case only: the initial pulse undergo modulation until a complete balance between nonlinearity and dispersion. In $2 \mathrm{~d}$ and higher dimensions, the solutions of (B.1) become instable on finite times due to Benjamin-Feir (modulation) instability, see 


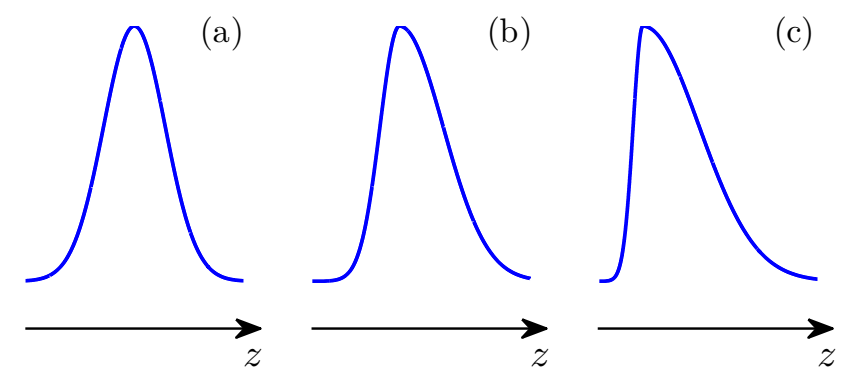

FiguRE B.1: Self-steepening and optical shock formation: (a) the incident optical pulse; (b) self-steepening of the wave profile after propagation; (c) formation of an optical shock wave, [24]

e.g. $[92,130]$.

In 1976 Glassey represented "the blow-up theorem" for the Cauchy problem for the NLSE in more general form [48]:

$$
\begin{aligned}
& \mathrm{i} \partial_{t} \psi=\triangle \psi+g|\psi|^{p-1} \psi, \quad x \in \mathbb{R}^{n}, \quad t>0 \\
& \psi(x, 0)=\phi(x),
\end{aligned}
$$

where $g>0, p>1$, and $\phi$ is assumed to be smooth and small on infinity. Then there exists a finite $T>0$, such that

$$
\lim _{t \rightarrow T^{-}}\|\nabla \psi(t)\|_{L^{2}}=+\infty
$$

provided that

$$
\int_{\mathbb{R}^{n}}\left(|\nabla \psi|^{2}-\frac{2 g}{p+1}|\psi|^{p+1}\right) d x=\text { const }=E_{0}<0
$$

and $p>1+4 / n$.

Thus, to study filament propagation, which is essentially a several-dimensional phenomenon, one needs to use models different from NLSE, such as NEE [26], UPPE [61] or MASP. 


\section{Appendix C}

\section{Proof of Lemma 2.1.1}

The proof of desired lemma follows [16] and [81] with adjustment on the case of BOA.

Proof. In the following, we will denote by $\mathcal{E}$ the laser operator:

$$
\mathcal{E}:\left(R_{0}, \mathbf{x}, t\right) \mapsto \mathcal{E}\left(R_{0}, \mathbf{x}, t\right)=\varrho\left(R_{0}, \mathbf{x}\right) \cdot \mathbf{E}(t),
$$

here $\mathbf{E}(t) \equiv \mathbf{E}\left(\mathbf{x}^{\prime}, t\right)$ as $\mathbf{x}^{\prime}$ is fixed, and a vectorial function $\varrho$ is defined as:

$$
\varrho:\left(R_{0}, \mathbf{x}\right) \mapsto \varrho\left(R_{0}, \mathbf{x}\right) \in \mathcal{C}_{b}^{2}\left(\mathbb{R}^{3}\right),
$$

in practice $\varrho$ can be constructed by convolution of $\mathbf{x}$ and a plateau function [79].

Using some $\varepsilon>0$, we then regularize the Coulomb potential, see (2.3), as following

$$
\begin{aligned}
V_{C}^{\varepsilon}\left(R_{0}, \mathbf{x}\right) & =-\left[\varepsilon+\left(x-\frac{R_{0}}{2} \cos \theta\right)^{2}+\left(y-\frac{R_{0}}{2} \sin \theta\right)^{2}+z^{2}\right]^{-1 / 2}- \\
- & {\left[\varepsilon+\left(x+\frac{R_{0}}{2} \cos \theta\right)^{2}+\left(y+\frac{R_{0}}{2} \sin \theta\right)^{2}+z^{2}\right]^{-1 / 2} }
\end{aligned}
$$

We have $\left|V_{C}^{\varepsilon}\right| \leq\left|V_{C}\right|$ and $\partial_{t} V_{C}^{\varepsilon}=0$. Then we can write the equation with regularized Coulomb and laser potentials, denoting the corresponding wavefunction as $\psi^{\varepsilon}$ :

$$
\mathrm{i} \partial_{t} \psi^{\varepsilon}\left(R_{0}, \mathbf{x}, t\right)=\left[\frac{1}{2} \triangle_{\mathbf{x}}+V_{C}^{\varepsilon}\left(R_{0}, \mathbf{x}\right)+\varrho\left(R_{0}, \mathbf{x}\right) \cdot \mathbf{E}(t)\right] \psi^{\varepsilon}\left(R_{0}, \mathbf{x}, t\right) .
$$


From [16], we can deduce that there exists a unique $\psi^{\varepsilon} \in \mathcal{C}^{0}\left(0, T ; H^{1} \cap H_{1}\right)$ solution to $(\mathrm{C} .2)$, as $V_{C}^{\varepsilon}$ and $\varrho \cdot \mathbf{E}$ belong to $L^{\infty}\left(0, T ; \mathcal{C}_{b}^{2}\left(\mathbb{R}^{3}\right)\right)$.

We now search for an estimate in $H_{1}$ of $\psi^{\varepsilon}$. A norm on $H^{1} \cap H_{1}$ is, for instance:

$$
\left\|\psi^{\varepsilon}(t)\right\|_{H^{1} \cap H_{1}}^{2}=\int_{\mathbb{R}^{3}}\left(\left|\nabla_{\mathbf{x}} \psi^{\varepsilon}\right|^{2}+\left(1+\left|\left(\mathbf{x}, \mathbf{R}_{0}\right)\right|^{2}\right)\left|\psi^{\varepsilon}\right|^{2}\right) d \mathbf{x},
$$

where $\left|\left(\mathbf{x}, \mathbf{R}_{0}\right)\right|^{2}:=\left|\mathbf{x}-\mathbf{R}_{0}\right|^{2}=\left|\mathbf{x}-\mathbf{e}_{0} R_{0}\right|^{2}$ with unit vector $\mathbf{e}_{0}$ that defines the orientation of the molecule. Then, there exists a constant $M_{p}>0$ such that:

$$
\int_{\mathbb{R}^{3}}\left[\frac{\left|\nabla_{\mathbf{x}} \psi^{\varepsilon}\right|^{2}}{2}+\left(1+\left|\left(\mathbf{x}, \mathbf{R}_{0}\right)\right|^{2}\right)\left|\psi^{\varepsilon}\right|^{2}\right] d \mathbf{x} \leq M_{p}\left\|\psi^{\varepsilon}(t)\right\|_{H^{1} \cap H_{1}}
$$

The idea of the proof:

- Find a positive constant $C$ such that:

$$
\left\|\psi^{\varepsilon}(t)\right\|_{H^{1} \cap H_{1}} \leq C\left\|\psi_{0}\right\|_{H^{1} \cap H_{1}} .
$$

- Use a compactness argument: there exists a sequence $\varepsilon_{n}$ such that

$$
\psi^{\varepsilon_{n}} \rightarrow_{n \rightarrow \infty}^{*} \psi \quad \text { in } \quad L^{\infty}\left(0, T ; H^{1} \cap H_{1}\right) .
$$

- From (C.5) and (C.6) we get

$$
\|\psi(t)\|_{H^{1} \cap H_{1}} \leq C\left\|\psi_{0}\right\|_{H^{1} \cap H_{1}} .
$$

This would prove the existence of a solution in the distributional sense to the TDSE (2.2). In order to obtain estimation (C.5), it is necessary to estimate

$$
\frac{d}{d t} \int_{\mathbb{R}^{3}}\left(1+\left|\left(\mathbf{x}, \mathbf{R}_{0}\right)\right|^{2}\right)\left|\psi^{\varepsilon}\right|^{2} d \mathbf{x}, \quad \text { and } \quad \frac{d}{d t} \int_{\mathbb{R}^{3}} \frac{\nabla_{\mathbf{x}} \psi^{\varepsilon}}{2} d \mathbf{x} .
$$


We multiply the TDSE (C.2) by $\left(1+\left|\left(\mathbf{x}, \mathbf{R}_{0}\right)\right|^{2}\right) \psi^{\varepsilon *}\left(\psi^{\varepsilon *}\right.$ is a c.c. of $\left.\psi^{\varepsilon}\right)$ and add with the complex conjugate TDSE multiplied by $-\left(1+\left|\left(\mathbf{x}, \mathbf{R}_{0}\right)\right|^{2}\right) \psi^{\varepsilon}$ :

$$
i \frac{d}{d t}\left(1+\left|\left(\mathbf{x}, \mathbf{R}_{0}\right)\right|^{2}\right)\left|\psi^{\varepsilon}\right|^{2}=\frac{1}{2}\left(1+\left|\left(\mathbf{x}, \mathbf{R}_{0}\right)\right|^{2}\right) \nabla_{\mathbf{x}}\left(\psi^{\varepsilon *} \nabla_{\mathbf{x}} \psi^{\varepsilon}-\psi^{\varepsilon} \nabla_{\mathbf{x}} \psi^{\varepsilon *}\right),
$$

where the RHS is also pure imaginary like the LHS. Then, we integrate the above expression on $\mathbb{R}^{3}$, and obtain, applying integration by parts and Cauchy-Schwarz inequality (for brevity of notation we omit the measure $d \mathbf{x}$ ):

$$
\begin{gathered}
\frac{d}{d t} \int_{\mathbb{R}^{3}}\left(1+\left|\left(\mathbf{x}, \mathbf{R}_{0}\right)\right|^{2}\right)\left|\psi^{\varepsilon}\right|^{2}=\int_{\mathbb{R}^{3}}\left[\nabla_{\mathbf{x}}\left(\left|\left(\mathbf{x}, \mathbf{R}_{0}\right)\right|^{2} \psi^{\varepsilon *}\right) \nabla_{\mathbf{x}} \psi^{\varepsilon}-\right. \\
\left.-\nabla_{\mathbf{x}}\left(\left|\left(\mathbf{x}, \mathbf{R}_{0}\right)\right|^{2} \psi^{\varepsilon}\right) \nabla_{\mathbf{x}} \psi^{\varepsilon *}\right]=\int_{\mathbb{R}^{3}} \nabla_{\mathbf{x}}\left|\left(\mathbf{x}, \mathbf{R}_{0}\right)\right|^{2}\left[\psi^{\varepsilon *} \nabla_{\mathbf{x}} \psi^{\varepsilon}-\psi^{\varepsilon} \nabla_{\mathbf{x}} \psi^{\varepsilon *}\right] \leq \\
\leq 2\left[\int_{\mathbb{R}^{3}}\left|\left(\mathbf{x}, \mathbf{R}_{0}\right)\right|^{2}\left|\psi^{\varepsilon}\right|^{2}\right]^{1 / 2}\left[\int_{\mathbb{R}^{3}}\left|\nabla_{\mathbf{x}} \psi^{\varepsilon}\right|^{2}\right]^{1 / 2} \leq \\
\leq \int_{\mathbb{R}^{3}}\left|\left(\mathbf{x}, \mathbf{R}_{0}\right)\right|^{2}\left|\psi^{\varepsilon}\right|^{2}+\int_{\mathbb{R}^{3}}\left|\nabla_{\mathbf{x}} \psi^{\varepsilon}\right|^{2} .
\end{gathered}
$$

Now we multiply (C.2) by $\partial_{t} \psi^{\varepsilon}$, and add with the complex conjugate TDSE multiplied by $\partial_{t} \psi^{\varepsilon *}$ :

$$
0=-\frac{1}{2}\left[\partial_{t} \psi^{\varepsilon *} \triangle_{\mathbf{x}} \psi^{\varepsilon}+\partial_{t} \psi^{\varepsilon} \triangle_{\mathbf{x}} \psi^{\varepsilon *}\right]+\left(V_{C}^{\varepsilon}+\mathcal{E}\right) \partial_{t}\left|\psi^{\varepsilon}\right|^{2} .
$$

Thus by integration by parts:

$$
\begin{array}{r}
\frac{d}{2 d t} \int_{\mathbb{R}^{3}}\left|\nabla_{\mathbf{x}} \psi^{\varepsilon}\right|^{2}=-\int_{\mathbb{R}^{3}}\left(V_{C}^{\varepsilon}+\mathcal{E}\right) \partial_{t}\left|\psi^{\varepsilon}\right|^{2}= \\
=-\frac{d}{d t} \int_{\mathbb{R}^{3}}\left(V_{C}^{\varepsilon}+\mathcal{E}\right)\left|\psi^{\varepsilon}\right|^{2}+\int_{\mathbb{R}^{3}}\left|\psi^{\varepsilon}\right|^{2} \partial_{t} \mathcal{E} .
\end{array}
$$

Obviously, we can bound the integral

$$
\int_{\mathbb{R}^{3}}\left|\psi^{\varepsilon}\right|^{2} \partial_{t} \mathcal{E} \leq C_{2}\left\|\partial_{t} \mathcal{E}\right\|_{L^{\infty}\left(\mathbb{R}^{3}\right)}\left\|\psi^{\varepsilon}\right\|_{H^{1} \cap H_{1}}^{2}
$$

with some $C_{2}>0$. 
Then following $[16,81]$, we set

$$
E^{\varepsilon}(t)=\int_{\mathbb{R}^{3}}\left[\frac{\left|\nabla_{\mathbf{x}} \psi^{\varepsilon}\right|^{2}}{2}+\left(1+\left|\left(\mathbf{x}, R_{0}\right)\right|_{L^{2}}^{2}\right)\left|\psi^{\varepsilon}\right|^{2}\right] \equiv\left\|\psi^{\varepsilon}(t)\right\|_{H^{1} \cap H_{1}}^{2}-\int_{\mathbb{R}^{3}} \frac{\left|\nabla_{\mathbf{x}} \psi^{\varepsilon}\right|^{2}}{2},
$$

and because of (C.9)-(C.11), there exists a positive constant $C_{3}$ such that

$$
\frac{d}{d t} E^{\varepsilon}(t) \leq \frac{d}{d t} \int_{\mathbb{R}^{3}}\left(\left(V_{C}^{\varepsilon}+\mathcal{E}\right)\left|\psi^{\varepsilon}\right|^{2}\right)+C_{3}\left[1+\left\|\partial_{t} \mathcal{E}\right\|_{L^{\infty}\left(\mathbb{R}^{3}\right)}\right] E^{\varepsilon}(t)
$$

By integration, and because of (C.9),(C.10),(C.11), there exists a positive constant $C_{3}$ such that

$$
\begin{gathered}
E^{\varepsilon}(t) \leq \int_{\mathbb{R}^{3}}\left(\left(V_{C}^{\varepsilon}+\mathcal{E}(t)\right)\left|\psi^{\varepsilon}(t)\right|^{2}\right)-\int_{\mathbb{R}^{3}}\left(\left(V_{C}^{\varepsilon}+\mathcal{E}(0)\right)\left|\psi^{\varepsilon}(0)\right|^{2}\right)+ \\
+C_{3} \int_{0}^{t}\left[1+\left\|\partial_{t} \mathcal{E}\right\|_{L^{\infty}\left(\mathbb{R}^{3}\right)}\right] E^{\varepsilon}(s) d s+E^{\varepsilon}(0)
\end{gathered}
$$

As by definition $V_{C}^{\varepsilon}$ (C.1) and $V_{C}$ (2.3) and applying Cauchy-Schwarz inequality:

$$
\begin{aligned}
\int_{\mathbb{R}^{3}}\left|V_{C}^{\varepsilon}\right|\left|\psi^{\varepsilon}(t)\right|^{2} & \leq \int_{\mathbb{R}^{3}}\left|V_{C}\right|\left|\psi^{\varepsilon}(t)\right|^{2} \leq \\
& \leq\left(\int_{\mathbb{R}^{3}} V_{C}^{2}\left|\psi^{\varepsilon}(t)\right|^{2}\right)^{1 / 2}\left(\int_{\mathbb{R}^{3}}\left|\psi^{\varepsilon}(t)\right|^{2}\right)^{1 / 2}
\end{aligned}
$$

As $V_{C}\left(\mathbf{x}, R_{0}\right)=\frac{1}{\left|\mathbf{x}-\mathbf{R}_{0}\right|}$, see $(2.3)$, then by applying Hardy's inequality in 3d-case with parameter $p=2$, there is a positive constant $C_{4}$ such that (using the definition of $\left.V_{C}\right)$

$$
\int_{\mathbb{R}^{3}} V_{C}^{2}\left|\psi(t)^{\varepsilon}\right|^{2} \leq C_{4} \int_{\mathbb{R}^{3}}\left|\nabla_{\mathbf{x}} \psi^{\varepsilon}\right|^{2}=C_{4}\left\|\nabla_{\mathbf{x}} \psi^{\varepsilon}\right\|_{L^{2}\left(\mathbb{R}^{3}\right)}^{2}
$$

So that, by the classical equality $\left\|\psi_{0}\right\|_{L^{2}}^{2}=\left\|\psi^{\varepsilon}\right\|_{L^{2}}^{2}$,

$$
\int_{\mathbb{R}^{3}}\left|V_{C}^{\varepsilon}\right|\left|\psi(t)^{\varepsilon}\right|^{2} \leq \frac{C_{4}}{2}\left\|\nabla_{\mathbf{x}} \psi^{\varepsilon}\right\|_{L^{2}\left(\mathbb{R}^{3}\right)}^{2}+\frac{C_{4}}{2}\left\|\psi_{0}\right\|_{L^{2}}^{2}
$$


Now as $\mathbf{E} \in L^{\infty}(0, T)$, and $\varrho \in C_{b}^{2}\left(\mathbb{R}^{3}\right)$

$$
\int_{\mathbb{R}^{3}}\left|\psi^{\varepsilon}\right|^{2} \mathcal{E}(t) \leq\|\mathcal{E}\|_{L^{\infty}\left(0, T ; \mathbb{R}^{3}\right)}\left\|\psi^{\varepsilon}\right\|_{H^{1} \cap H_{1}}^{2}
$$

and because of (C.4) and definition for $E^{\varepsilon}(t)$, see (C.12),

$$
E^{\varepsilon}(0) \leq M_{p}\left\|\psi_{0}\right\|_{H^{1} \cap H_{1}}^{2}
$$

and by definition $V_{C}$ there exists a positive constant $C_{5}$ such that

$$
\int_{\mathbb{R}^{3}}\left(\left(V_{C}^{\varepsilon}+\mathcal{E}(0)\right)\left|\psi^{\varepsilon}(0)\right|^{2}\right) \leq C_{5}\left\|\psi_{0}\right\|_{H^{1} \cap H_{1}}^{2}
$$

Then (C.13)-(C.16), lead to the existence of 2 positive constants $C_{6}, C_{7}$ such that:

$$
\left\|\psi^{\varepsilon}(t)\right\|_{H^{1} \cap H_{1}}^{2} \leq C_{6}\left\|\left.\psi_{0}\right|_{H^{1} \cap H_{1}} ^{2}+C_{7} \int_{0}^{t}\left[1+\left\|\partial_{t} \mathcal{E}\right\|_{L^{\infty}\left(0, T ; \mathbb{R}^{3}\right)}\right]\right\| \psi^{\varepsilon}(s) \|_{H^{1} \cap H_{1}}^{2} d s .
$$

We apply to (C.17) Grönwall's inequality with a positive constant $C_{8}$ :

$$
\left\|\psi^{\varepsilon}(t)\right\|_{H^{1} \cap H_{1}}^{2} \leq C_{8} \exp \left(\int_{0}^{t}\left[1+\left\|\partial_{t} \mathcal{E}\right\|_{L^{\infty}\left(0, T ; \mathbb{R}^{3}\right)}\right] d s\right)\left\|\psi_{0}\right\|_{H^{1} \cap H_{1}}^{2} .
$$

Finally, as $\partial_{t} \mathbf{E}\left(\mathbf{x}^{\prime}, \cdot\right) \in L^{1}(0, T)$, there exists a constant $C$ such that (C.5) occurs. 


\section{Bibliography}

[1] M.V. Ammosov, N.V. Delone, and V.P. Krainov. Tunnel ionization of complex atoms and atomic ions in electromagnetic field. Sov. Phys. JETP, 91:2008-2013, 1986.

[2] Ph. Antoine, A. L'Huillier, M. Lewenstein, Salières P., and B. Carré. Theory of high-harmonic generation by an elliptically polarized laser field. Phys. Rev. A, 53(3):1725-1745, 1996.

[3] X. Antoine and C. Besse. Unconditionally stable discretization schemes of nonreflecting boundary conditions for one-dimentional Schrödinger equation. J. of Comput. Phys., 188:157-175, 2003.

[4] G. Askar'yan. Effects of the gradient of a strong electromagnetic beam on electrons and atoms. Sov. Phys. JETP, 15(6):1088-1090, 1962.

[5] I. Babushkin and L. Bergé. The fundamental solution of the unidirectional pulse propagation equation. J. of Math. Phys., 55(032903), 2014.

[6] Ph. Balcou, P. Salières, A. L'Huillier, and M. Lewenstein. Dynamic imaging of nuclear wavefunctions with ultrashort UV laser pulses. Phys. Rev. A, 55(4):3204-3210, 1997. 
[7] A. Bandrauk, S. Barmaki, S. Chelkowski, and G.L. Kamta. Molecular high order harmonic generation. Progress in Ultrafast Intense Laser Science, edited by K.Yamanouchi et al. (Springer, NY), III(Chap.9), 2007.

[8] A. Bandrauk and K.-J. Yuan. Circularly polarized attosecond pulses and molecular atto-magnetism. In the book From Atomic to Mesoscale, Chapter 10, edited by S. Malinovskaya and I. Novikova (World Scientific), pages 207-220, 2015.

[9] A.D. Bandrauk, S. Barmaki, and G. Lagmago Kamta. Laser phase control of HHG. the $\mathrm{H}^{+}-\mathrm{H}_{2}^{+}$system. Phys. Rev. Lett., 98(013001), 2007.

[10] A.D. Bandrauk, S. Chelkowski, and S. Goudreau. Control of harmonic generation using two-colour femtosecond-attosecond laser fields: quantum and classical perspectives. J. Mod. Opt., 52(411), 2005.

[11] A.D. Bandrauk, S. Chelkowski, and H.S. Nguyen. Nonlinear photon processes in molecules at high intensities-route to XUV- attosecond pulse generation. $J$. Molec. Str., 735C:203-2009, 2005.

[12] A.D. Bandrauk, E. Lorin, and J.V. Moloney, editors. Laser Filamentation. Mathematical Methods and Models. Springer, CRM Series in Mathematical Physics edition, 2016.

[13] A.D. Bandrauk and H.Z. Lu. Controlling harmonic generation in molecules with intense laser and static magnetic fields: Orientation effects. Phys. Rev. A, 68(4):043408/1-043408/7, 2003.

[14] A.D. Bandrauk and H.S. Nguyen. Attosecond control of ionization and highorder harmonic generation in molecules. Phys. Rev. A, 66(031401), 2002. 
[15] A.D. Bandrauk, H. Yu, S. Chelkowski, and E. Constant. Enhanced harmonic generation in extended molecular systems by two-color excitation. Phys. Rev. A, 56(2537), 1997.

[16] L. Baudouin, O. Kavan, and J.-P Puel. Regularity for a Schrödinger equation with singular potential and application to bilinear optimal control. J. Diff. Equ., 216:188-222, 2005.

[17] P. Béjot, E. Cormier, E. Hertz, B. Lavorel, J. Kasparian, J.-P. Wolf, and O. Faucher. High-field quantum calculation reveals time-dependent negative kerr contribution. Phys. Rev. Lett., 110(4), 2013.

[18] P. Béjot, J. Kasparian, S. Henin, V. Loriot, T. Vieillard, E. Hertz, O. Faucher, B. Lavorel, and J.-P. Wolf. Higher-order Kerr terms allow ionization-free filamentation in gases. Phys. Rev. Lett., 104(10):103903, Mar 2010.

[19] C.M. Bender and S.A. Orszag. Advanced Mathematical Methods for Scienticts and Engineers I. Asymptotic Methods and Perturbation Theory. Springer, NY, 2nd edition, 1999.

[20] L. Bergé, C. Gouédard, J. Schjodt-Eriksen, and H. Ward. Filamentation patterns in Kerr media vs. beam shape robustness, nonlinear saturation and polarization states. Physica D, 176:181-211, 2003.

[21] L. Bergé, S. Skupin, R. Nuter, J. Kasparian, and J.-P. Wolf. Ultrashort filaments of light in weakly ionized, optically transparent media. Reports on Progress in Phys., 70(10):1633-1713, 2007.

[22] V.I Bespalov and V.I Talanov. About filamentation of beams of light in a nonlinear liquid. JETP Lett., 3:307-310, 1966. 
[23] B. Bidégaray-Fesquet. Hiérarchie de modèles en optique quantique. De MaxwellBloch à Schrödinger non-linéaire. Springer-Verlag, Berlin, Mathématiques et Applications, vol. 49 edition, 2006.

[24] R. W. Boyd. Nonlinear Optics. Academic Press, 3rd edition, 2008.

[25] R.W. Boyd. Order-of-magnitude estimates of the nonlnear optical susceptibility. J. of Mod. Optics, 46(3).

[26] T. Brabec and F. Krausz. Nonlinear optical pulse propagation in the single-cycle regime. Phys. Rev. Lett., 78:3282-3285, 1997.

[27] T. Brabec and F. Krausz. Intense few-cycle laser fields: Frontiers of nonlinear optics. Rev. Mod. Phys., 72:545-591, Apr 2000.

[28] A. Braun, G. Korn, X. Liu, D. Du, J. Squier, and G. Mourou. Self-channeling of high-peak-power femtosecond laser pulses in air. Optics Lett., 20(1):73-75, 1995.

[29] W. Cao, P. Lu, P. Lan, X. Wang, and G. Yang. Single-attosecond pulse generation with an intense multicycle driving pulse. Phys. Rev. A, 74(063821), 2006.

[30] S. Champeaux, L. Bergé, D. Gordon, A. Ting, J. Peano, and P. Sprangle. $(3+1)$-dimensional numerical simulations of femtosecond laser filaments in air: Toward a quantitative agreement with experiments. Phys. Rev. E, 77(3), 2008.

[31] S. Chelkowski, C. Foisy, and A. Bandrauk. Electron-nuclear dynamics of multiphoton $\mathrm{H}_{2}^{+}$dissociative ionization in intense laser field. Phys. Rev. A, 57(2):1176-1185, 1998.

[32] F.F. Chen. Introduction to plasma physics and controlled fusion. V.1: Plasma Physics. Plenum Press. New York, 2nd edition, 1984. 
[33] S.L. Chin. Femtosecond Laser Filamentation. Springer, 2010.

[34] E.R. Cohen and P. Giacomo. Symbols, Units, Nomenclature and Fundamental Constants in Physics. International Union of Pure and Applied Physics Commission C2. SUNAMCO 87-1, 2010, URL: http://iupap.org/wpcontent/uploads/2014/05/A4.pdf.

[35] P.B. Corkum. Plasma perspective on strong-field multiphoton ionization. Phys. Rev. Lett., 71(13):1994-1997, 1993.

[36] P.B. Corkum and F. Krausz. Attosecond science. Nature Phys., 3(381), 2007.

[37] O. Costin, R.D. Costin, and J.L. Lebowitz. Nonperturbative time dependent solution of a simple ionization model. arXiv:1706.07129, 2017, URL:https://arxiv.org/abs/1706.07129.

[38] A. Couairon and A. Mysyrowicz. Organizing multiple femtosecond filaments in air. Phys. Report., 41(3):47-189, 2007.

[39] E. Cunningham and Z. Chang. Optical gating with asymmetric field ratios for isolated attosecond pulse generation. IEEE Journal on Selected Topics in Quantum Electronics, 21(5), 2015.

[40] F. De Vuyst. Numerical modeling of tramsport problems using freefem++ software - with examples in biology, CFD, traffic flow and energy transfer. HAL Id: cel-00842234, 2013, URL: https://cel.archives-ouvertes.fr/cel-00842234.

[41] P. Ding, S. Mitryukovskiy, A. Houard, E. Oliva, A. Couairon, A. Mysyrowicz, and Y. Liu. Backward lasing of air plasma pumped by circularly polarized femtosecond pulses for the sake of remote sensing (BLACK). Opt. Express, 22(24):29964-29977, 2014. 
[42] J. Dongarra, A. Lumsdaine, R. Pozo, and K. Remington. A Sparse Matrix Library in $\mathrm{C}++$ for High Performance Architectures. Proceedings of the Second Object Oriented Numerics Conference, pages 214-218, 1994, URL: http://math.nist.gov/sparselib++/.

[43] E: Dumas, D. Lannes, and J. Szeftel. Variants of focusing nls equation: Derivation, justification, and open problems related to filamentation. In the Book Laser Filamentation. Mathematical Methods and Models, edited by E. Lorin et al. (Springer), pages 19-75, 2016.

[44] M.Y. Emelin and M.Y. Ryabikin. Basics of attosecond physics (in Russian). Nizhni Novgorod State University, URL: http://www.unn.ru/pages/ranking/method/oaf.pdf, 2014.

[45] M. Ferray, A. L'Huillier, X. F. Li, L. A. Lompre, G. Mainfray, and C. Manus. Multiple-harmonic conversion of $1064 \mathrm{~nm}$ radiation in rare gases. J. of Phys. B, 21(3):L31-L35, 1988.

[46] M.B. Gaarde, M. Murakami, and R. Kienberger. Spatial separation of large dynamical blueshift and harmonic generation. Phys. Rev. A, 74(053401), 2006.

[47] J.-Y. Ge and Z.H. Zhang. Use of negative complex potential as absorbing potential. J. Chem. Phys., 108(4):1429-1432, 1998.

[48] R.T. Glassey. On the blowing up of solutions to the Cauchy problem for nonlinear Schrödinger equations. J. Math. Phys., 18(9):1794-1797, 1977.

[49] C. Grossmann, H.-G. Roos, and M. Stynes. Numerical Treatment of Partial Differential Equations. Springer, 2nd, Universitext edition, 2007. 
[50] A.V. Husakou and J. Herrmann. Supercontinuum generation of higher-order solitons by fission in photonic crystal fibers. Phys. Rev. Lett., 87(20):203901/1$203901 / 4,2001$.

[51] R.J. Iório Jr. and D. Marchesin. On the schrödinger equation with timedependent electric fields. Proc. Roy. Soc. Edinburg Sect., A96(1-2), 1984.

[52] J. Itatani, J. Levesque, D. Zeidler, and et al. Tomographic imaging of molecular orbitals. Nature, 432(7019):867-871, 2004.

[53] M.Yu. Ivanov and P.B. Corkum. Generation of high-order harmonics from inertially confined molecular ions. Phys. Rev. A, 48(1):580-590, 1993.

[54] M.Yu. Ivanov, P.B. Corkum, T. Zuo, and A. Bandrauk. Routes to control of intense-field atomic polarizability. Phys. Rev. Lett., 74(15):2933-2936, 1995.

[55] V.P. Kalosha and J. Herrmann. Self-phase modulation and compression of fewoptical-cycle pulses. Phys. Rev. A, 62:011804(R), 2000.

[56] A. Kamor, C. Chandre, T. Uzer, and F. Mauger. Recollision scenario without tunneling: Role of the ionic core potential. Phys. Rev. Lett., 112(13), 2014.

[57] T. Kanai, S. Minemote, and H. Sakai. Quantum interference during high-order harmonic generation from aligned molecules. Nature, 435:470, 2005.

[58] L.V. Keldysh. Ionization in field of a strong electromagnetic wave. Sov. Phys. JETP, 20(5):1307, 1965.

[59] C. Köhler, R. Guichard, E. Lorin, S. Chelkowski, A.D. Bandrauk, L. Bergé, and S. Skupin. Saturation of the nonlinear refractive index in atomic gases. Phys. Rev. A, 87(4), 2013.

[60] W. Kohn and L.J. Sham. Self-consistent equations including exchange and correlation effects. Phys. Rev., 140(A1133), 1965. 
[61] M. Kolesik and J.V. Moloney. Nonlinear optical pulse propagation simulation: From maxwell's to unidirectional equations. Phys. Rev. E, 70(3 2):036604-1036604-11, 2004.

[62] M. Kolesik and J.V. Moloney. Modeling and simulation techniques in extreme nonlinear optics of gaseous and condensed media. Reports on Progress in Phys., $77(016401), 2014$.

[63] M. Kolesik, E. M. Wright, and J.V. Moloney. Femtosecond filamentation in air and higher order nonlinearities. Opt. Expess, 35(15), 2010.

[64] J.L. Krause, K.J. Schafer, and K.C. Kulander. High-order harmonic generation from atoms and ions in the high intensity regime. Phys. Rev. Lett., 68(24):35453538, 1992.

[65] E.A. Kuznetsov, S.L. Musher, and A.V. Shafarenko. Collapse of acoustic waves in media with positive dispersion. JETP Lett., 37(5):241, 1983.

[66] G. Lagmago Kamta and A.D. Bandrauk. Imaging molecular orbitals with ultrashort intense laser pulses. Phys. Rev. A, 74(033415), 2006.

[67] P. Lan, P. Lu, W. Cao, X. Wang, and G. Yang. Phase-locked high-orderharmonic and sub-100-as pulse generation from stretched molecules. Phys. Rev. A, 74(063411), 2006.

[68] L.D. Landau and E.M. Lifshitz. Classical Theory of Fields. Course of Theor. Phys., V.2, in Russian. Nauka, Moscow, 7rd corrected edition, 1988.

[69] L.D. Landau and E.M. Lifshitz. Quantum mechanics: Non-relativistic theory. Course of Theor. Phys., V.3, in Russian. Nauka, Moscow, 4rd corrected edition, 1989. 
[70] L.D. Landau and E.M. Lifshitz. Electrodinamic of Continuous Media. Course of Theor. Phys., V.8, in Russian. Nauka, Moscow, 3rd corrected edition, 1992.

[71] H. Leblond, H. Triki, and Mihalache. Circularly polarized few-optical-cycle solutions in the short-wave-approximation regime. Phys. Rev. A, 84(023833), 2011.

[72] M. Lein. Molecular imaging using recolliding electrons. J. Phys. B, 40(R135), 2007.

[73] M. Lewenstein, Ph. Balcou, M.Yu. Ivanov, A. L'Huillier, and P.B. Corkum. Theory of high-harmonic generation by low-frequency laser fields. Phys. Rev. A, 49(3):2117-2132, 1994.

[74] S. Long, W. Becker, and J.K. Mciver. Model-calculations of polarizationdependent 2-colar high-harmonic generation. Phys. Rev. A, 52(2262), 1995.

[75] E. Lorin and A. Bandrauk. Efficient parallel computing for laser-gas quantum interaction and propagation. $22^{\text {th }}$ High Performance Computing Symposium, IEEE, pages 4-8, 2008.

[76] E. Lorin and A. Bandrauk. A maxwell-schrödinger-plasma model and computing aspects for intense, high frequency and ultrashort laser-gas interaction. Proceedings of 23rd Internat. Symp., HPCS 2009, Kingston, ON, Canada, pages 70-75, 2009.

[77] E. Lorin and A.D. Bandrauk. Efficient and accurate numerical modeling of a micro-macro nonlinear optics model for intense and short laser pulses. J. of Comput. Sci., 3(3):159-168, 2012.

[78] E. Lorin, S. Chelkowski, and A. Bandrauk. A Maxwell-Schrodinger model for non-perturbative laser-molecule interaction and some methods of numerical 
computation. In High-dimensional partial differential equations in science and engineering, volume 41 of CRM Proc. Lecture Notes, pages 161-182. Amer. Math. Soc., Providence, RI, 2007.

[79] E. Lorin, S. Chelkowski, and A. Bandrauk. A numerical Maxwell-Schrödinger model for laser-matter interaction and propagation. Comput. Phys. Comm., 177(12):908-932, 2007.

[80] E. Lorin, S. Chelkowski, and A. Bandrauk. Attosecond pulse generation from aligned molecules - dynamics and propagation in $\mathrm{H}_{2}^{+}$. New J. Phys., 10(025033):21pp, 2008.

[81] E. Lorin, S. Chelkowski, and A. Bandrauk. The WASP model: A micro-macro system of wave-Schrödinger-plasma equations for filamentation. Commun. in Comput. Phys., 9(2):406-440, 2011.

[82] E. Lorin, S. Chelkowsky, E. Zaoui, and A.D. Bandrauk. Maxwell-SchroedingerPlasma (MASP) model for laser-molecule interactions: towards quantum filamentation with intense ultrashort pulses. Physica D, 241(12):1059, 2012.

[83] E. Lorin, M. Lytova, and A.D. Bandrauk. Nonperturbative nonlinear maxwellschrödinger models for intense laser pulse propagation. In the Book Laser Filamentation. Mathematical Methods and Models, edited by E. Lorin et al. (Springer), pages 167-183, 2016.

[84] E. Lorin, M. Lytova, A. Memarian, and A.D. Bandrauk. Development of nonperturbative nonlinear optics models including effects of high order nonlinearities and of free electron plasma: Maxwell-Schrödinger equations coupled with evolution equations for polarization effects, and the sfa-like nonlinear optics model. J. of Phys. A, Math. and Theor. Phys., 48(105201), 2015. 
[85] M. Lytova, E. Lorin, and A. D. Bandrauk. Propagation of intense and short circularly polarized pulses in a molecular gas: From multiphoton ionization to nonlinear macroscopic effects. Phys. Rev. A, 94:013421, Jul 2016.

[86] N.L. Manakov and V.D. Ovsyannikov. Measurement of high order kerr refractive index of major air components. Sov. Phys. JETP, 52:895, 1980.

[87] R.C. McOwen. Partial Differential Equations : methods and applications. Pearson Education, Inc, Upper Saddle River, New Jersey 07458, 2003.

[88] A. McPherson, G. Gibson, H. Jara, U. Johann, T. S. Luk, I. A. McIntyre, K. Boyer, and C. K. Rhodes. Studies of multiphoton production of vacuumultraviolet radiation in the rare gases. J. of the Opt. Soc. of Am. B, 4(4):595$601,1987$.

[89] L. Mediauskas, J. Wragg, H. van der Hart, and M.Yu Ivanov. Generating isolated elliptically polarized attosecond pulses using bichromatic counterrotating circularly polarized laser fields. Phys. Rev. Lett., 115(15):153001, Oct 2015.

[90] T. Morishita, A.-T. Le, Z. Chen, and C. D. Lin. Accurate retrieval of structural information from laser-induced photoelectron and high-order harmonic spectra by few-cycle laser pulses. Phys. Rev. Lett., 100(1):013903, Jan 2008.

[91] G. Mur. Absorbing boundary conditions for te finite-difference approximation of the time-domain electromagetic-field equations. IEEE Trans. Electromagn. Compatibility, EMC-23:377-382, 1981.

[92] A.C. Newell. Solitons in Mathematics and Physics. Society for Industrial and Applied Mathematics, 1987. 
[93] A.C. Newell. Short pulse evolution equation. In the Book Laser Filamentation. Mathematical Methods and Models, edited by E. Lorin et al. (Springer), pages $1-17,2016$.

[94] A.C. Newell and J.V. Moloney. Nonlinear Optics. Addison-Wesle Publishing Company, advanced topics in the interdisciplinary mathematical sciences edition, 1992.

[95] J.M. Ngoko Djiokap, S.X. Hu, L.B. Madsen, N.L. Manakov, A.V. Meremianin, and A.F. Starace. Electron vortices in photoionization by circularly polarized attosecond pulses. Phys. Rev. Lett., 115(11), 2015.

[96] H.S. Nguyen, A. Suda, and K. Midorikawa. Generation and propagation of attosecond pulses in He gas with sub-10-fs driver pulses. Phys. Rev. A, 60(3), Sept. 1999.

[97] J.C. Painter, M. Adams, N. Brimhall, and et al. Direct observation of laser filamentation in high-order harmonic generation. Opt. Lett., 31(3471), 2006.

[98] P. Panagiotopoulos, P.T. Whalen, M. Kolesik, and J.V. Moloney. Numerical simulation of ultra-short laser pulses. In the Book Laser Filamentation. Mathematical Methods and Models, edited by E. Lorin et al. (Springer), pages 185-213, 2016.

[99] P. Polynkin, M. Kolesik, E.M. Wright, and J. Moloney. Experimental tests of the new paradigm for laser filamentation in gases. Phys. Rev. Lett., 106, 2011.

[100] R. Pozo, K. Remington, and A. Lumsdaine. Sparselib++ v. 1.5. Sparse Matrix Class Library. Reference guide. 1996, URL: http://math.nist.gov/sparselib++/sparselib-userguide.pdf. 
[101] A. Quarteroni, R. Sacco, and F. Saleri. Numerical Mathematics. Springer Berlin Heidelberg, Texts in Applied Mathematics, 2nd edition, 2007.

[102] B. Quesnel and P. Mora. Theory and simulation of the interaction of ultraintense laser pulses with electrons in vacuum. Phys. Rev. E, 58(3):3719, 1998.

[103] M. Richter, S. Patchkovskii, F. Morales, O. Smirnova, and M. Ivanov. The role of the Kramers-Henneberger atom in the higher-order Kerr effect. New J. of Phys., 15(083012), 2013.

[104] N.M. Ryskin and D.I. Trubetskov. Nonlinear waves., in Russian. Nauka, PhysMatLit, Moscow, the modern theory of oscillations and waves edition, 2000.

[105] K.J. Schafer, B. Yang, L.F. Dimauro, and K.C. Kulander. Above threshold ionization beyond the high harmonic cutoff. Phys. Rev. Lett., 70(11):1599-1602, 1993.

[106] Y.R. Shen. The Principles of Nonlinear Optics. John Wiley \& Sons, a wileyinterscience publication edition, 1984.

[107] S. Skupin and L. Bergé. Self-guiding of femtosecond light pulses in condensed media: Plasma generation versus chromatic dispersion. Physica D, 220(1):14$30,2006$.

[108] A. Soffer and M.I. Weinstein. Ionization and scattering for short-lived potentials. Lett. in Math. Phys., 48:339-352, 1999.

[109] A. Spott, A. Jaro-Becker, and A. Becker. Ab initio and perturbative calculations of the electric susceptibility of atomic hydrogen. Phys. Rev. A, 90, 2014.

[110] A. Stathopoulos and J.R. McCombs. PRIMME: PReconditioned Iterative MultiMethod Eigensolver: Methods and software description. ACM Transactions on Mathematical Software, 37(2):21:1-21:30, 2010. 
[111] G. Strang. On the construction and comparison of difference schemes. SIAM J. Numer. Anal., 5:506-517, 1968.

[112] V.V. Strelkov. High-order optical processes: towards nonperturbative nonlinear optics. arXiv:1504.07871 [physics.atom-ph], 2015, URL: http://arxiv.org/pdf/1504.07871v1.pdf.

[113] J. Strikwerda. Finite Difference Schemes and Partial Differential Equations. Society for Industrial and Applied Mathematics (SIAM), Philadelphia, PA, 2nd edition, 2004.

[114] C. Sulem and P.-L. Sulem. The Nonlinear Schrödinger Equation. Self-Focusing and Wave Collapse. Springer-Verlag New York, Applied Mathematical Sciences edition, 1999.

[115] M.E Taylor. Partial Differential Equations I. Basic Theory. Springer, 2nd edition, 2011.

[116] F. Théberge, N. Aközbek, W. Liu, and et al. Tunable ultrashort laser pulses generated through filamentation in gases. Phys. Rev. Lett., 97(023904), 2006.

[117] F. Théberge, J. Filion, N. Aközbek, Y. Chen, A. Becker, and S.L. Chin. Selfstabilization of third-harmonic pulse during two-color filamentation in gases. Appl. Phys. B, 87(2):207-210, 2007.

[118] R. Velotta, N. Hay, M.B. Mason, and et al. High-order harmonic generation in aligned molecules. Phys. Rev. Lett., 87(18), 2001.

[119] A. Vinçotte and L. Bergé. Atmospheric propagation of gradient-shaped and spinning femtosecond light pulses. Physica D, 223(2):163-173, 2006. 
[120] C. Vozzi, F. Calegari, E. Benedetti, and et al. Controlling two-center interference in molecular high harmonic generation. Phys. Rev. Lett., 95(153902), 1995.

[121] G.B. Whitham. Linear and Nonlinear Waves. John Wiley \& Sons, New York, Wiley-Interscience Publication edition.

[122] L. Wu, E. Romero, and A. Stathopoulos. Primme_svds: A high-performance preconditioned SVD solver for accurate large-scale computations. arXiv, 1607.01404, 2016, URL: http://arxiv.org/abs/1607.01404.

[123] K.S. Yee. Numerical solution of initial boundary value problem involving maxwell's equations in isotropic media. IEEE Trans. Antennas Propag., AP14:302-307, 1966.

[124] H.-M. Yin. Existence and regularity of a weak solution to Maxwell's equations with a thermal effect. Math. Methods Appl. Sci., 29(10):1199-1213, 2006.

[125] K.-J. Yuan and A.D. Bandrauk. High-order elliptically polarized harmonic generation in extended molecules with ultrashort intense bichromatic circularly polarized pulses. Phys. Rev. A, 81(063412), 2010.

[126] K.-J. Yuan and A.D. Bandrauk. Circularly polarized molecular high-order harmonic generation in $\mathrm{H}_{2}^{+}$with intense laser pulses and static field. Phys. Rev. A, 83(063422), 2011.

[127] K.-J. Yuan, S. Chelkowski, and A.D. Bandrauk. Molecular photoelectron angular distribution rotation in multi-photon resonant ionization of $\mathrm{H}_{2}^{+}$by circularly polarized ultraviolet laser pulses. J. of Chem. Phys., 142(144303), 2015. 
[128] K.J. Yuan and A.D. Bandrauk. Circularly polarized attosecond pulses from molecular high-order harmonic generation by ultrashort intense bichromatic circularly and linearly polarized laser pulses. J. of Phys. B, 45(074001), 2012.

[129] G. L. Yudin, S. Patchkovskii, P.B. Corkum, and A.D. Bandrauk. Attosecond photoelectron interference in the separable coulomb-volkov continuum. J. Phys. B, 40:F93, 2007.

[130] V.E. Zakharov and L.A. Ostrovsky. Modulation instability: The beginning. Physica D, 238:540-548, 2009.

[131] T. Zuo and A.D. Bandrauk. Phase control of molecular ionization: $\mathrm{H}_{2}^{+}$and $\mathrm{H}_{3}^{++}$in intense two-color laser fields. Phys. Rev. A, 54(4):3254-3260, Oct 1996. 